\title{
OPERATIONAL ANALYSIS AND SAFETY AUDIT FOR EAST YORK
} TRAFFIC NETWORK

$$
\text { by }
$$

Sumit Bhasin, B.Eng. (Civil), Chandigarh, India, June 2003

A project

presented to Ryerson University

in partial fulfillment of the

requirements for the degree of

Master of Engineering

in the program of

Civil Engineering

Toronto, Ontario, Canada, 2007

(C) Sumit Bhasin 2007 


\section{Author's Declaration}

I hereby declare that I am the sole author of this project.

I authorize Ryerson University to lend this project to other institutions or individuals for the purpose of scholarly research.

I further authorize Ryerson University to reproduce this project by photocopying or by other means, in total or in part, at the request of other institutions or individuals for the purpose of scholarly research. 


\title{
OPERATIONAL ANALYSIS AND SAFETY AUDIT FOR EAST YORK TRAFFIC NETWORK
}

\author{
By
}

\section{Sumit Bhasin}

Submitted to the Department of Civil Engineering at Ryerson University, Toronto, Ontario on May $16^{\text {th }}, 2007$ in partial fulfillment of the requirements for the degree of Master of Engineering in Civil Engineering, 2007

Project Supervisor: Dr. Bhagwant Persaud

\begin{abstract}
Enhancing the quality of road travel is one of the main challenges present day traffic engineers and planners face. Travel delays cause loss of millions of person hours each year; and the economic toll of road accidents is staggering. Thus, engineers are always looking at opportunities for reducing delays and accidents. This project evaluates the operational and safety deficiency in a traffic network of nine intersections in the East York region and recommends appropriate and feasible corrective measures. The first phase of the project deals with the evaluation of traffic operations in the network, using simulation and optimization techniques, while the second phase encompasses a road safety audit that attempts to reduce crashes and fatalities.
\end{abstract}

The study reveals that the majority of the intersections in the network are failing operationally, with level of service (LOS) F typical. Although a reduction in delays is achieved by optimization, no substantive improvement in LOS can be obtained by optimization alone. It is recommended that geometry and operations of the critical intersections be altered to enhance quality of service. Analysis of collision data was supported by a site investigation; recommendations for improving safety include relocating traffic signs, improving pavement condition and lighting and installing additional traffic control devices. 


\section{Acknowledgements}

I am grateful to all who have given their whole-hearted support and assistance during the course of this project. I would like to thank my project supervisor, Dr. Bhagwant Persaud, for his guidance and motivation during this project and throughout my academic years. Dr. Bhagwant Persaud has been very supportive ever, since I started my degree at Ryerson. I shall always remain deeply indebted to him and shall make sure I do him proud.

A special thanks to Mr. Blair Lagden, Transportation Services - City of Toronto, for providing me with the links and documents that were so important for this project. I thank all my professors at Ryerson University for their co-operation and encouragement. Sincere thanks to Dr. Ali Mekky, Dr. Songnian Li, Dr. Khaled Sennah and Dr. Paul Poh for all their support.

I would like to thank my parents for their love and encouragement and would like to dedicate this project to them. They have been my support system and have acknowledged every small accomplishment of mine.

Also, I wish to express my gratitude and sincere thanks to my fellow students and friends for all their support. I would like to express my appreciation and regard for a very special friend, Faisal Siddiqui. He has been my mentor and I attribute my gainful and pleasant experience at Ryerson to him and all whom I have associated with during the course of my degree. 


\section{Table of Contents}

Title Page -...-

Author's Declaration -.

Abstract-_-_-_o- iii

Acknowledgements - - iv

Table of Contents -

List of Figures - - vi

List of Tables --__n viii

List of Appendices-1x

1.0 Introduction- 1

1.1 Objective -... 2

1.2 Site Selection-2

1.3 Report Organization -..... 4

2.0 Literature review - 5

2.1 Case Studies - 5

2.2 Traffic Operations - 10

2.3 Transyt - 12

2.4 Synchro - 13

2.5 Safety Audit-n- 14

3.0 Network Analysis \& Optimization -

3.1 Site Features -

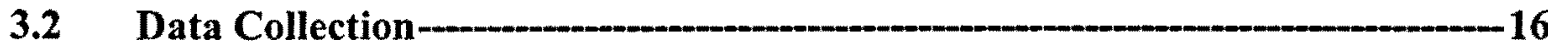

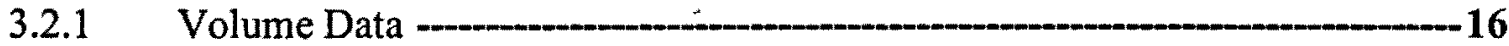

3.2.2 Collision Data - 17

3.3 Methodology-_-18

3.4 Analysis - 19

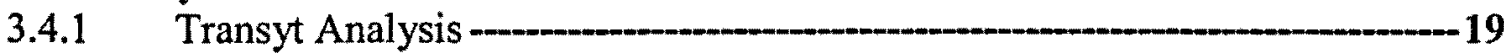

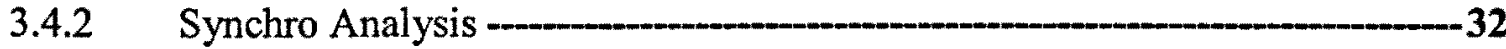

4.0 Audit Report \& Observations

4.1 Collision Analysis-___- 36

4.2 Site visit-_._-

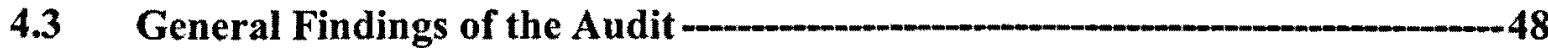

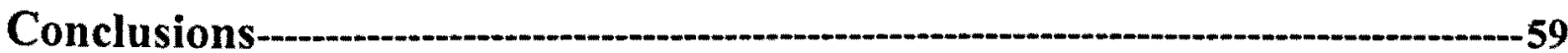

References - 
Figure 1: Network representation (www.earth.google.com) 3

Figure 2: Roundabout Case Study 3

Figure 3: Land use designations (City of Toronto- Planning Division) 15

Figure 4: Synchro \& Transyt comparison chart $\quad 32$

Figure 5: Synchro street network showing traffic volumes 33

Figure 6: Synchro street network showing CF and street names $\quad 34$

Figure 7: Collisions by year - Coxwell \& Mortimer 36

Figure 8: Collisions by hour- Coxwell \& Mortimer 36

Figure 9: Collisions by classification - Còxwell \& Mortimer 37

Figure 10: Collisions by class of collision- Coxwell \& Mortimer 37

Figure 11: Collisions by year - Coxwell \& Cosburn 38

Figure 12: Collisions by hour - Coxwell \& Cosburn 38

Figure 13: Collisions by classification- Coxwell \& Cosburn 39

Figure 14: Collisions by class of collision- Coxwell \& Cosburn 39

Figure 15: Collisions by year - Coxwell \& O'Connor $\quad{ }^{\prime} \quad 40$

Figure 16: Collisions by hour - Coxwell \& O'Connor 40

Figure 17: Collisions by classification - Coxwell \& O'Connor 41

Figure 18: Collisions by class of collision - Coxwell \& O'Connor 41

Figure 19: Collisions by year - O'Connor \& Don Mills 42

Figure 20: Collisions by hour - O'Connor \& Don Mills 42

Figure 21: Collisions by classification -O'Connor \& Don Mills 43

Figure 22: Collisions by class of collision - O'Connor \& Don Mills 43

Figure 23: Collisions by year - O' Connor \& Donlands 44

Figure 24: Collisions by hour - O' Connor \& Donlands 44

Figure 25: Collisions by classification - O' Connor \& Donlands 45

Figure 26: Collisions by class of collision - O' Connor \& Donlands 45

Figure 27: Collisions by hour- Cosburn \& Donlands 46

Figure 28: Collisions by hour - Cosburn \& Donlands 46

Figure 29: Collisions by classification - Cosburn \& Donlands . 47

Figure 30: Collisions by class of collision - Cosburn \& Donlands 47

Figure 31: Road side parking at Coxwell \& Mortimer 49

Figure 32: Road side parking at Coxwell \& Mortimer $\quad 49$

Figure 33: No provision for bus bay - Coxwell \& Mortimer 49

Figure 34: No provision for bus bay - Coxwell \& Mortimer 49

Figure 35: Sign hidden by overgrown tree - Coxwell \& Cosburn $\quad 50$

Figure 36: Student pick up \& drop-off area at Coxwell \& Cosburn 50

Figure 37: View of Coxwell \& Cosbum intersection 51

Figure 38: No bus bay provided at Coxwell \& Cosburn 51

Figure 39: Speed limit sign needs relocation- Coxwell \& O'Connor 52

Figure 40: No bus bay at Coxwell \& O'Connor 52

Figure 41: Entry/ Exit from gas station at Coxwell \& O'Connor 53

Figure 42: Entry/ Exit from gas station at Coxwell \& O'Connor 53

Figure 43: Queues at O'Connor \& Don Mills 54

Figure 44: O'Connor \& Don Mills 54 
Figure 45: Pavement needs reconditioning at O'Connor \& Don Mills 55

Figure 46: Signs need relocation - O'Connor \& Don Mills 55

Figure 47: Signs need to be relocated - O' Connor \& Donlands 56

Figure 48: Pot holes and cracks at $O^{\prime}$ Connor \& Donlands $\quad 56$

Figure 49: Congestion - O' Connor \& Donlands 57

Figure 50: Long queues close to O' Connor \& Donlands $\quad 57$

Figure 51: No bus bays at Cosburn \& Donlands $\quad 58$

Figure 52: View of Cosburn \& Donlands intersection $\quad 58$ 
Table 1: Eastbound Speed Profile $\quad 8$

Table 2: Westbound Speed Profile $\quad 8$

Table 3: Transyt Results- Network Performance Summary 19

Table 4: Base Case Analysis Results- Coxwell \& Mortimer 20

Table 5: Results after Split Optimization- Coxwell \& Mortimer 20

Table 6: Results after Cycle Length Optimization- Coxwell \& Mortimer 20

Table 7: Base Case Analysis Results- Coxwell \& Cosburn 21

Table 8: Results after Split Optimization- Coxwell \& Cosburn 21

Table 9: Results after Cycle Length Optimization- Coxwell \& Cosburn 21

Table 10: Base Case Analysis Results - Coxwell \& Plains 22

Table 11: Results after Split Optimization- Coxwell \& Plains 22

Table 12: Results after Cycle Length Optimization- Coxwell \& Plains 22

Table 13: Base Case Analysis Results- Coxwell \& O' Connor 23

Table 14: Results after Split Optimization- Coxwell \& O' Connor 23

Table 15: Results after Cycle Length Optimization- Coxwell \& O' Connor 23

Table 16: Results after changes in lane configuration- Coxwell \& O' Connor 24

Table 17: Base Case Analysis Results - O' Connor \& Don Mills 25

Table 18: Results after Split Optimization- O' Connor \& Don Mills 25

Table 19: Results after Cycle Length Optimization- O' Connor \& Don Mills 25

Table 20: Base Case Analysis Results - O' Connor \& Donlands 26

Table 21: Results after Split Optimization- O' Connor \& Donlands 26

Table 22: Results after Cycle Length Optimization- O' Connor \& Donlands $\quad 26$

Table 23: Results after changes in lane configuration- O' Connor \& Donlands 27

Table 24: Base Case Analysis Results- Cosburn \& Donlands 28

Table 25: Results after Split Optimization- Cosburn \& Donlands 28

Table 26: Results after Cycle Length Optimization- Cosburn \& Donlands 28

Table 27: Base Case Analysis Results- Cosburn \& Greenwood 29

Table 28: Results after Split Optimization- Cosburn \& Greenwood 29

Table 29: Results after Cycle Length Optimization- Cosburn \& Greenwood 29

Table 30: Base Case Analysis Results - Mortimer \& Greenwood $\quad 30$

Table 31: Results after Split Optimization- Mortimer \& Greenwood 30

Table 32: Results after Cycle Length Optimization- Mortimer \& Greenwood 30

Table 33: Results after changes in lane configuration- Mortimer \& Greenwood 31

Table 34: Synchro Results- Network Performance Summary 32 


\section{List of Appendices}

$\begin{array}{ll}\text { Appendix A } & \text { Transyt Report } \\ \text { Appendix B } & \text { Synchro Report }\end{array}$ 


\subsection{Introduction}

Transportation infrastructure represents one of the largest and most critical investments made today. The movement of goods and people is vital to social and economic development of a nation. The number of vehicles on the roads is ever increasing whereas the roads and the land available for building new roads is very limited. Managing, redirecting and decongesting traffic within the existing roads is a challenging task that traffic engineers and planners face today.

To streamline traffic-flow, reduce delays and make travel safer and more convenient for the daily road-user, we first need to identify the problem. Performance measurement provides critical information that helps detect potential problems and forms the basis for enforcing corrective measures ${ }^{(1)}$.

The process of safety improvement involves network screening, site visits, collision and operational analysis. Delays at intersections are a major source of traffic congestion and in order to minimize vehicular delays at intersections, signal timings and phasing needs to be properly designed. Since manual analysis of traffic networks is a cumbersome and monotonous task, use of software is common in this era of technological advancement.

Traffic operations' software tools available today have a wide range of applications; encompassing highway capacity analysis procedures, simulation for evaluation of the impact of changing traffic patterns, network optimization, traffic impact studies and geometric designs.

For the road network, infrastructure performance is measured in three main categories: physical condition, operational efficiency and safety. 


\section{$1.1 \quad$ Objective}

The aim of this project is to perform operational analysis and safety audit of a congested network of nine intersections in the East York region. The major area of interest in the network is the heavily congested Don Mills and O'Connor intersection where large inflow and outflow of traffic occurs to and from Don Valley Parkway. Commuters from outskirts of Toronto mostly use Don Valley Parkway to reach the Central Business District/ Centreville of city of Toronto. As a result Don Valley Parkway and the Don Mills - O'Connor intersection is mostly found to be operating at capacity.

In this research I have made an effort to enhance safety and improve upon the level of service which will in turn provide reduction in fuel consumption and average delays faced by daily commuters. Transyt and Synchro software have been used for simulation and optimization of the network. Site visits and analysis of collision data for last five years was performed in an effort to improve safety.

\subsection{Site Selection}

The selected road network comprises of nine signalized intersections situated in the congested area of East York, Toronto.

East York is a former suburb of Toronto. The area is populated with middle-class and workingclass homes and is a major arrival point for immigrants, who have established their first Canadian residence in the Thorncliffe Park apartments ${ }^{(6)}$.

The chosen network is bounded by Donlands Avenue on West, Coxwell Avenue on East, Mortimer Avenue on South and O'Connor on North.

This site evolves a lot of interest for research because of its proximity to Don Valley Parkway. Don Valley Parkway is one of Toronto's busiest commuter routes which was built as part of a : grand plan initiated in the 1950 s. The highways plan never got completed because of downtown 
objections to several of the expressway routes, leaving DVP and the Gardiner Expressway to carry the bulk of highway traffic into the core ${ }^{(5)}$.

The population of the suburbs has grown tremendously eversince and has had a toll on the volumes on the DVP, resulting in frequent congestion. Don Valley Parkway and the Don Mills O'Connor intersection in our network is a focus area where bottlenecks are common and large scale attention is required for alleviating the situation.

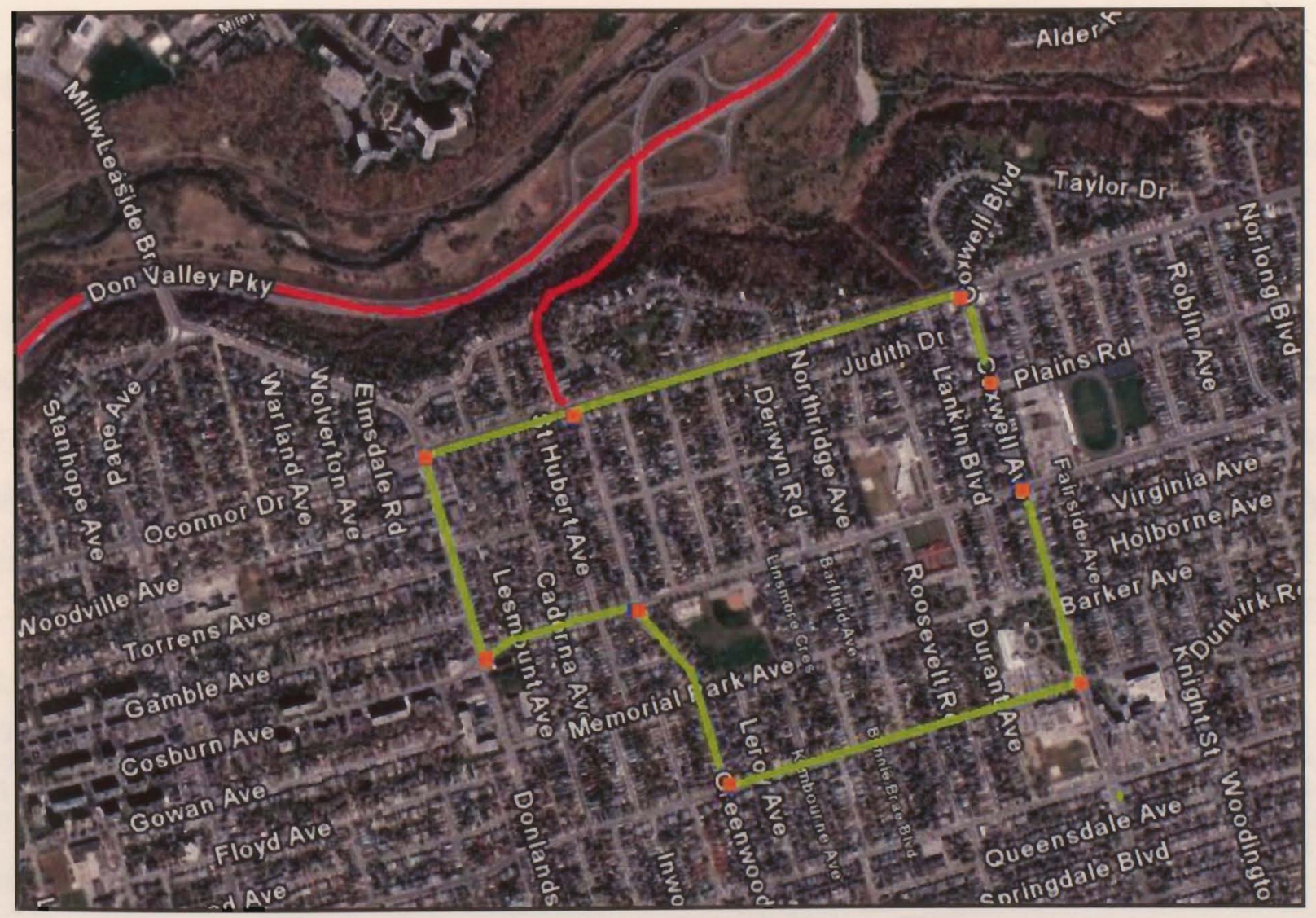

Figure 1: Network representation (www.earth.google.com)

Figure shows the proximity of Don Valley Parkway to the network. The DVP has been marked in red, the network in green and the intersections have been shown in orange. 


\subsection{Report Organization}

This report is organized in four chapters; Introduction, Literature Review, Network Analysis and Optimization, Audit Report and Observations; followed by Conclusion and References.

Chapter 1 provides a brief introduction to the project, its purpose and the relevance of taking up the East York traffic network as the area of interest.

Chapter 2 summarizes the literature reviewed for the research. It includes case studies that consolidate the significance of providing treatments to the network, followed by overview of traffic operations and safety audit and brief introduction of software used in the project.

Chapter 3 discuses site features, presents volume data and collision data provided by the City of Toronto and details the methodology adopted. Network Analysis follows which provides summary of simulation and optimization results. Software analysis showed that little could be done to improve existing level of service as the traffic volumes are very high. Options for improvement included changing lane configuration, signal timings and phases; the results for which are summarized individually in the result analysis section of this report.

Chapter 4 presents the details of site investigation, backed up with pictures and graphical analysis of collision data. The section also describes findings of the safety audit followed by recommendations.

The report finishes with the conclusions and references. 


\subsection{Literature review}

The purpose of measuring performance is to improve transportation services for customers ${ }^{(9)}$. It is a useful tool that can help inform the public as well as decision makers and legislators regarding the importance and the merits of making appropriate investments in the transportation system ${ }^{(8)}$. For the road network, the traffic engineers measure highway infrastructure performance in three main categories: physical condition, functional adequacy and utilization ${ }^{(9)}$.

To support the purpose of this research, following cases were studied and it was concluded that optimization techniques when employed at appropriate sites result in improving level of service and corrective measures at accident prone sites improve safety of commuters.

The first portion of this report focuses on Level of Service analysis for which simulation modeling and optimization programs have been employed. Simulation techniques replicate conditions of the road and thus help in analyzing variety of complex vehicle interactions and evaluation of alternative treatments. The outputs obtained from the software are interpreted by traffic engineers and improvements are implemented.

\subsection{Case Studies}

In 2003, Al-Ghamdi ${ }^{(2)}$ investigated a total of 1774 police-reported traffic accidents that occurred in the period 1996-1998 in Riyadh. Analysis depicted that about 50\% of severe accidents involved a pedestrian, indicating the need for protecting pedestrians in Riyadh.

The analysis of accident causes in this study revealed that excess speed, failing to yield and disregarding the red light accounted for majority of all accident causes. The author concluded that reviewing and correcting existing intersection geometry for defects at the crash sites backed by strict law enforcement strategies and public education campaigns would help bring a reduction in crashes. 
In 1997, Persaud et al. ${ }^{(10)}$ studied the impact of converting signalised one-way street intersections in Philadelphia to all-way stop sign intersections. Empirical Bayesian procedure was used to estimate what would have been the impact on number of crashes if the intersections were not converted. On comparison of the Empirical Bayesian estimates with actual crash numbers after conversion, a $24 \%$ reduction in crashes was achieved. For all crash types, significant reductions were achieved for both day \& night and percentage reduction in crashes involving severe injuries was substantially larger than those resulting in minor injuries.

In 2006 , Sudani ${ }^{(11)}$ conducted a road safety audit for a high risk corridor in the region of Waterloo, Ontario. The audit involved a detailed study of the accidents in that corridor for years 2000-2005. Site investigation and in depth examination revealed the shortcomings in the geometric design and suggested relocation of traffic signs, improvement of lighting and installation of traffic control devices like red light cameras be done to improve safety.

In 2002, Abbas ${ }^{(1)}$ provided an assessment of traffic safety conditions for rural roads in Egypt. The author presented an analysis of accidents' causes which were categorized under six main categories, namely; driver related, pedestrian related, vehicle related, road related, environmentrelated causes and other causes. In the course of conducting the traffic safety assessment of rural roads in Egypt, the researcher suggested the lack of past sustainable and detailed accident data collection programs as well as a lack of accident prediction models. The author in his research developed a number of statistical models that could be utilized for prediction of the expected number of accidents, injuries, fatalities and casualties on the rural roads in Egypt.

In 2001 , Eccles and Hummer ${ }^{(7)}$ evaluated the safety effects of replacing existing yellow signs with fluorescent yellow warning signs at seven hazardous locations. A before and after study that used surrogate measures like signal violations, stopping behavior and speed, was conducted and it was found that use of fluorescent yellow sheeting in place of standard yellow sheeting provides an inexpensive method that increases conspicuity of the signs and helps increase safety. A 
substantial reduction in crashes was achieved at four of the seven sites while at the other three, little change was observed. However, since surrogate measures were used, actual collision savings was unknown. The authors recommended further research to find collision savings and long term effects.

In 2007, Ourston ${ }^{(4)}$ conducted a case study on roundabouts constructed at Meadowbrook Drive/Hamilton Drive in the village of Ancaster (Hamilton) Ontario, Canada.

The objective was to reduce speeds on Wilson Street; the roundabout was constructed, between the $50 \mathrm{~km} / \mathrm{h}$ (30 mph) speed limit in the village and the $80 \mathrm{~km} / \mathrm{h}(50 \mathrm{mph})$ approach from Highway 403 to the west. A spot speed study was conducted to determine speed profiles eastbound and westbound on Wilson Street at six locations before and after the roundabout was opened.

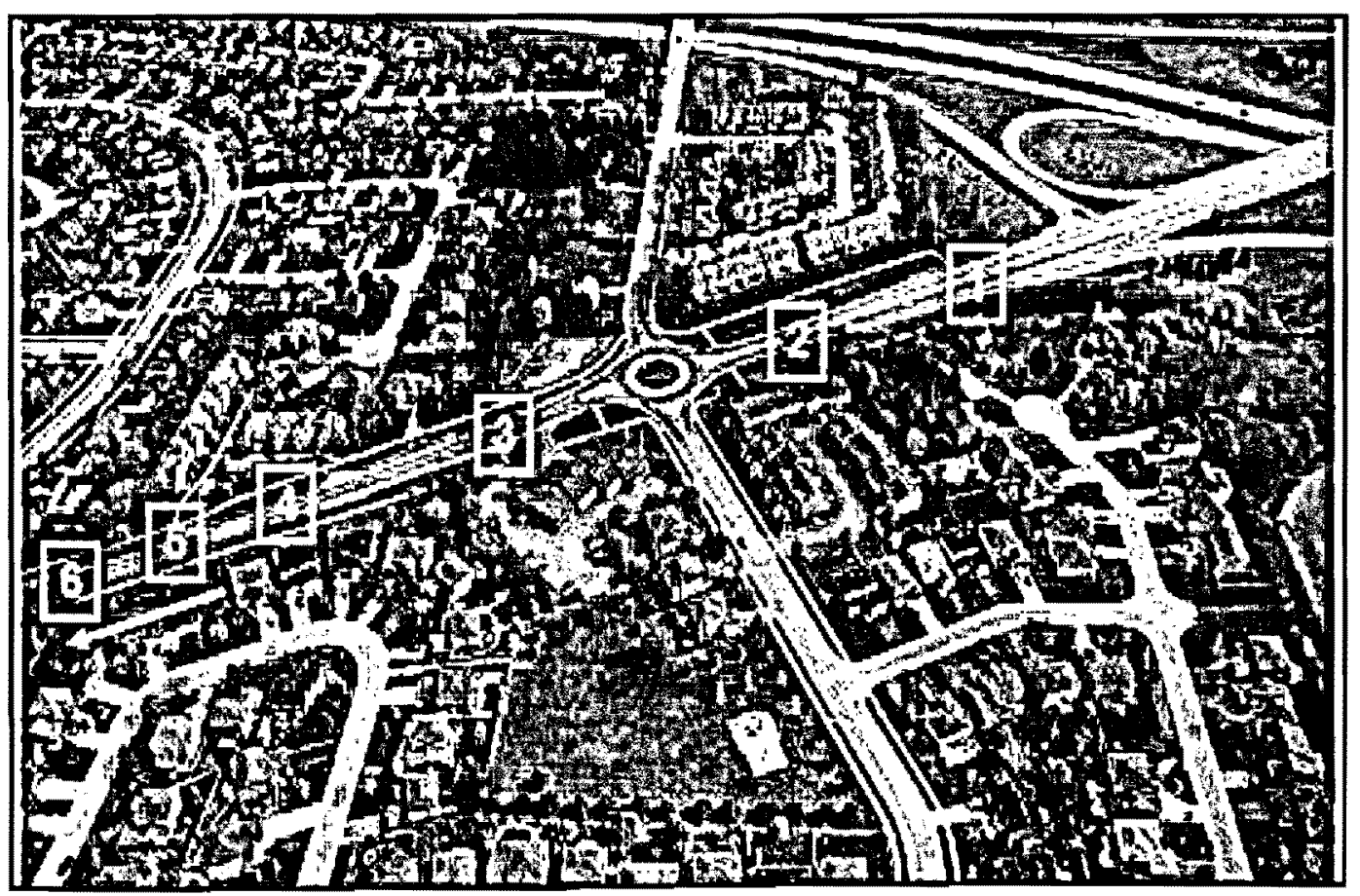

Figure 2: Roundabout Case Study 


\begin{tabular}{|c|c|c|c|}
\hline Location & Before Speed $(\mathrm{km} / \mathrm{h})$ & After Speed $(\mathrm{km} / \mathrm{h})$ & Difference $(\mathrm{km} / \mathrm{h}) \mid$ \\
\hline Sput 1 & 78 & 63 & -15 \\
\hline Spot 2 & 76 & -32 & -34 \\
\hline & \multicolumn{3}{|c|}{ Roundabout } \\
\hline Spot 3 & 74 & -45 & -29 \\
\hline Spot 4 & 70 & 60 & -10 \\
\hline Spot 5 & 67 & 68 & $+!$ \\
\hline Spot 6 & 67 & 67 & 0 \\
\hline
\end{tabular}

Table 1: Eastbound Speed Profile

\begin{tabular}{|c|c|c|c|}
\hline Spot 6 & 69 & 66 & -3 \\
\hline Spot 5 & 70 & 68 & -2 \\
\hline Spot 4 & 76 & 58 & -18 \\
\hline Spot 3 & 80 & 39 & -41 \\
\hline \multicolumn{4}{|c|}{ Roundabout } \\
\hline Spot 2 & 77 & 52 & -25 \\
\hline Spot 1 & 77 & 58 & -19 \\
\hline
\end{tabular}

Table 2: Westbound Speed Profile

The study demonstrated that roundabout significantly reduced speeds eastbound on the highspeed approach before motorists reached the village and westbound as they leave it. Roundabouts eliminate the potential for hazardous conflicts such as right-angle and left-turn head-on crashes and have fewer conflict points compared to conventional intersections. Lower speeds in roundabouts allow drivers more time to react to potential conflicts, helping to make roundabouts safer. Highly visible signs and markings, conspicuous central landscaping, and adequate illumination are also recommended to reduce approach speeds and crash potential at roundabouts. 
In 2005, Bauer et al. ${ }^{(3)}$ conducted a before/after study of same-direction, four- or five-lane urban freeways in California using the Empirical Bayes Method. The authors inferred that relieving congestion on an urban freeway by widening the existing roadbed to add an additional lane is a difficult and expensive option, however, re-striping the existing lanes, converting all or part of the shoulder to a travel lane, or a combination of both options is more practical. The study found that the accident frequency in four- to five-lane conversions was 10 to 11 percent, while smaller increases were demonstrated in five- to six-lane conversions. The authors attributed the increases in accident frequency to accident migration caused by relocation of traffic operational bottlenecks. 


\subsection{Traffic Operations}

Traffic operations imply management of traffic on the road which encompasses controlling vehicles moving in conflicting directions by means of traffic signals, at the same time ensuring that no unnecessary delay occurs.

Traffic signals are control devices which can alternately direct the traffic to stop and proceed at intersections using red, yellow and green traffic light signals. The signals provide for orderly movement of traffic, increase the traffic handling capacity of intersections, reduce crashes, and increase the safety of pedestrian crossings.

The main objective of signal timing at the intersections is to reduce the average delays of all vehicles and the probability of crashes. The objective of reducing delay sometimes conflicts with the idea of crash prevention because number of phases need to be minimized for reduction of average delay, however, many additional distinct phases are required to eliminate conflicting movements of vehicles. Hence engineering judgment and experience needs to be resorted to at these times.

Traffic signals can operate in several different modes:

- Pre-timed Signal Control

- Traffic Actuated Signal Control

- Semi-Actuated Control

- Fully Actuated Control

Pre-timed signals are the simplest type of traffic signals in which the cycle length, the phases and all of the intervals are predetermined. Traffic-actuated control of intersections attempts to adjust green time continuously to handle fluctuating volumes. These adjustments occur in accordance with real-time measures of traffic demand obtained from vehicle detectors placed on the approaches to the intersection. Fully-actuated signals have detectors on all of the approaches while semi-actuated signals have detectors on only some of the approaches. 
To elaborate on the mechanism of actuated signal control; when the detector registers a vehicle it makes a call to the controller. The controller then adjusts the phase lengths to meet the requirements of the prevailing traffic condition. When the detector is activated, it retains the right of way for a minimum time and allots additional time, if more cars are detected during the green light. Extensions are added to the phase, till it reaches the maximum green time. However, if no call is received during the green time, the phase ends. The cycle then progresses based on calls received and changes to the next phase in the phase sequence that has a call.

Another important aspect in traffic control is that of signal coordination. Coordinating signal timing allows a system of signals to work together so that vehicles are able to move through the signals without stopping. Good signal coordination can generate measurable safety benefits in two main ways. Firstly; coordinated signals produce platoons of vehicles that can proceed without stopping at multiple consecutive signalized intersections. Reducing the number and . frequency of required stops and maintaining constant speeds for all vehicles reduce rear-end conflicts. Secondly; signal coordination can improve the operation of turning movements. Increased platooning can create more gaps of greater lengths for vehicle movements at intersections and can result in improved intersection operation which means reduced energy consumption and lesser pollution. 


\subsection{Transyt}

TRANSYT-7F (TRAffic Network StudY Tool, version 7, Federal) is complete traffic signal timing optimization software for traffic networks which can cater extremely complex traffic conditions. TRANSYT has the capability of optimizing cycle length, phasing sequence, splits, and offsets. The program accepts user inputs on signal timing and phase sequences, geometric conditions, operational parameters, and traffic volumes ${ }^{(13)}$.

To elaborate the data input procedure; we divide roads and lanes into links with shared signals and shared traffic movements, assign a saturation flow to each link, indicate the maximum traffic flow over the stop line during the green time and enter the signal timings that apply to each link. The output includes the maximum capacity, the degree of saturation, the maximum queue length during the cycle for each of the links.

For simulation, the program takes the inputs as fixed variables and reports the performance measures in terms of stops, delay, fuel consumption, and queuing.

Transyt can also optimise signal timings over the network to reduce delays, stops, and fuel consumption and total operating cost. When optimizing, TRANSYT-7F minimizes or maximizes an objective function, called the Performance Index (PI). The PI may be a combination of delay and stops; fuel consumption; and/or optionally selected excessive maximum back of queue, excess operating costs, or progression opportunities ${ }^{(13,16)}$.

TRANSYT-7F is also available in both DOS and Windows 95/NT versions. 


\subsection{Synchro}

Synchro is a traffic signal timing tool designed to optimize cycle lengths, splits, offsets, and phase orders. Synchro requires mostly the same traffic flow and geometric data as Transyt-7F. The program can be used to evaluate existing traffic signal timing or to optimize settings for individual intersections, arteries, or a network.

The program performance measures include average approach delay, intersection delay, volumeto-capacity ratio, intersection level of service, total stops, travel time, emissions, and fuel consumption. Synchro offers a variety of user-specified reports, including capacity analysis, LOS, delay, stops, fuel consumption and signal timing settings ${ }^{(13)}$.

Synchro's unique visual displays, including an interactive platoon dispersion diagram allow the user to can change the offsets and splits with a mouse, and observe the impacts on delay, stops, and LOS for the individual intersections, as well as the entire network.

Further, the program also optimizes multiple cycle lengths and performs coordination analysis in which Synchro determines which intersections need to be coordinated and those that should run free. The decision process is based on an analysis of each pair of adjacent intersections to determine what is called the "Coordinatability Factor" (CF) for the links between them. Synchro's "Coordinatability Analysis Report" shows each factor that affects CF along with the effect it has on the CF. CF ranges from 0 to 100 or more. Any value above 50 means that coordination is recommended. The higher the $\mathrm{CF}$, the more likely that segment will benefit from coordination.

Another significant strength of Synchro is its ability to create data input streams for Transyt-7F and CORSIM. Once the user has entered the data to run Synchro successfully, it is possible to run any one of these programs without using any of their pre-processors ${ }^{(13)}$.

Synchro runs under Windows 95/NT and OS/2. 


\subsection{Safety Audit}

Traffic accidents are a cause of concern world-wide. Massive sums of money are being spent on emergency care, rehabilitation, and other costs that result from traffic collisions (Health Canada estimates that Canada spends $\$ 25$ billion every year on costs related to road collisions). Besides the monetary expense, road crashes cause loss of life and suffering as many are left crippled for rest of their lives. The World Health Organization reports that an estimated million people are killed and over forty million injured around the world, every year due to traffic collisions.

Policy makers, road safety professionals and engineers are working to reduce the risk and cost of collisions and are conducting road safety audits that aim at identification of safety problems and suggestion of corrective measures.

Road Safety Audit entails an in-depth engineering study of a road using road safety principles with the purpose of identifying cost-effective countermeasures that would improve safety and operations for all road users ${ }^{(15)}$. Safety audits are generally most effective when conducted at locations where a high collision risk has been identified.

To elaborate the methodology of conducting an audit; it includes a comprehensive review of collision history, geometric characteristics, and traffic operational efficiency, and could also include traffic conflict observations and a human factors assessment. 


\subsection{Network Analysis \& Optimization}

Network Analysis includes determination of traffic operational efficiency. Simulation and Optimization software like Transyt and Synchro have been used in this research to determine Level of Service of the intersections in the network. Before elaborating on the methodology and discussion of analysis's results, I shall first provide a brief overview of the features of the site along with the sources from where the data has been acquired.

\subsection{Site Features}

Land-use in the region is mostly dominated by closely packed residences. Land areas are occupied by apartments, mixed-housing and small-business neighborhoods. Immediately north of the region, there is a huge employment area north of Overlea and south of Eglinton, and there are mixed-use areas south of the region, all along and south of Danforth as depicted in the figure below. More than half the houses built are apartments and rest is a mix of row houses and semidetached units. The region has schools and a hospital. Land use and community design have had an impact on mode choice. Auto remains the preferred mode of travel. As a result of the current scenario, with increasing rate of population and employment growth in the region existing roadway facilities are quite insufficient.

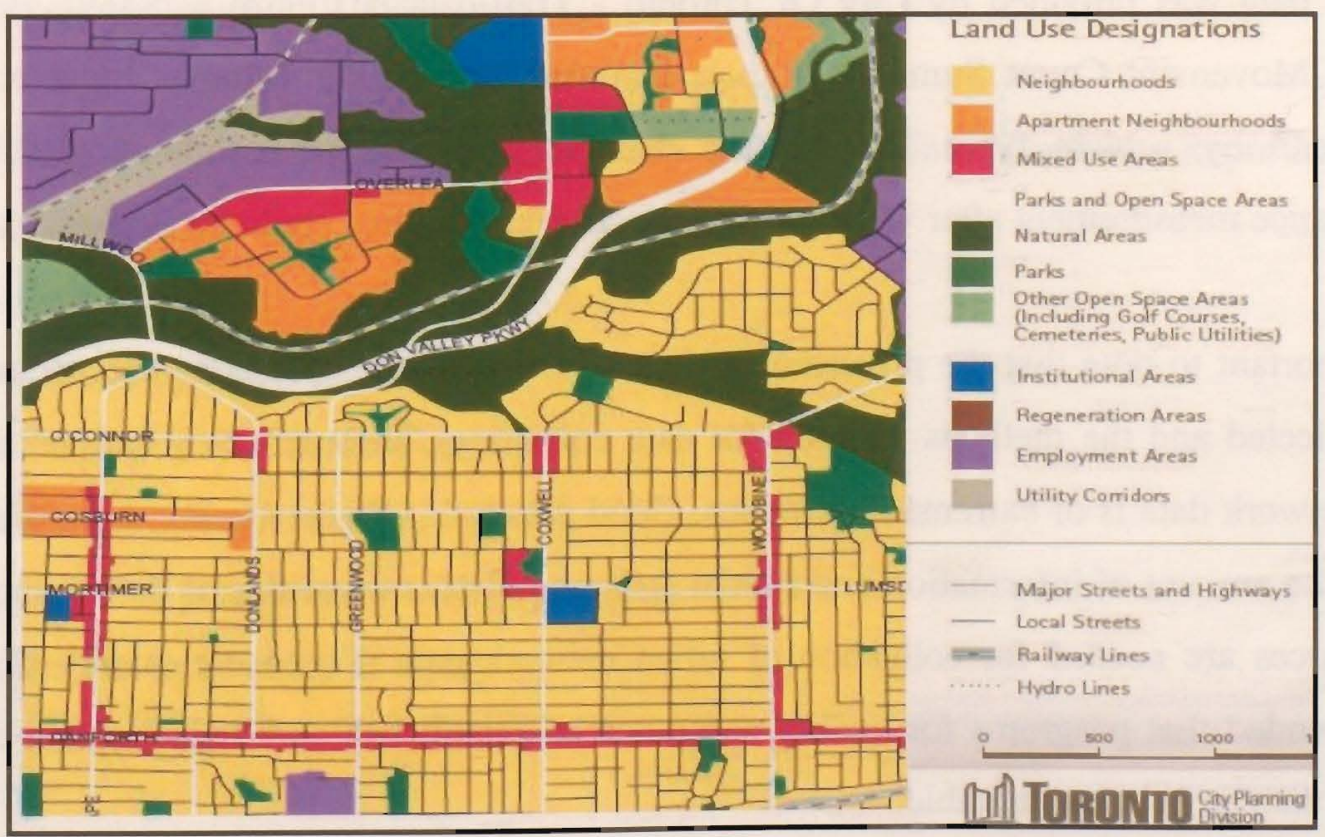

Figure 3: Land Use Designations (City of Toronto- Planning Division) 


\subsection{Data Collection}

The site of selected road network was inspected for collection of signal timing data during P.M. peak period. Road geometry \& lane configurations were also observed in the field. Cycles were observed for each intersection. From the field survey, it was observed that all of the signals are pre-timed with two splits. The signal cycle length was observed to be 100 seconds for all intersections. The traffic data pertaining to the volume, composition of vehicles and pedestrians was taken from the records of City of Toronto.

Distance between intersection to intersection was not field measured and was taken from help online. For speed limit, posted limits on the streets were coded. For major streets $50 \mathrm{~km} / \mathrm{hr}$ was used. For the minor streets, $40 \mathrm{~km} / \mathrm{hr}$. was used. For the unsignalized intersections, traffic volumes were not provided by the City of Toronto. For the purpose of coding, traffic volumes at the unsignalized intersections were calculated by balancing the traffic from the nearest intersections.

\subsubsection{Volume Data}

Volume data was provided by City Of Toronto - Traffic Data Centre \& Safety Bureau. The Turning Movement Count Summary Report included intersection volumes by direction, time period and by vehicle type. The volume data was fed into the simulation software for performance measurement after which corrective countermeasures could be implemented.

It is important to note that the precision of the calculated LOS depends on the accuracy of the data collected and the methods adopted for data collection. Thus accuracy and detail of urban street network data is of extreme importance. Good data holds the key to good decision making; Due to the amount of information and spatial \& temporal characteristics of data, a large amount of resources are needed for collection of urban network data. To ensure quality of data, it is recommended that programs for quality assurance be established and agencies that collect and manage data be held accountable. 


\subsubsection{Collision Data}

Traffic collisions involve complex interaction between vehicles, human behavior and environmental conditions. Factors responsible for a collision can be many; such as error in judgment, geometrical deficiency, roadway condition or environment condition. Corrective measures can only be exercised upon careful analysis of collision data and conclusions should be drawn upon appropriate qualification and supporting information.

The collision data are categorized into five types, namely;

- General collision,

- Angle -collision,

- Rear-end collision,

- Left-turn collision and

- Pedestrian collision

These collisions are also categorized into three levels of severity;

- Fatal,

- Non-fatal injury and

- Property damage only (PDO)

- Collision Data for the research was provided by City of Toronto- Collision Reporting System and included details of:

- Collisions by year

- Collisions by month of the year

- Collisions by road surface condition

- Collisions by impact type

- Category of person involved by age group

- Collisions by severity of injury 


\subsection{Methodology}

Level of service is measured in reference to delay at an intersection taking all turning volumes into account. It is the fundamental factor that contributes to the congestion and overall network performance. Transportation engineers determine measures to minimize the delay by analyzing and designing proper phasing, signal timing and lane configuration at the intersections. There are various tools and software available today to assist engineers in their effort to enhance the road network performance. This project represents the analysis of a typical heavily congested East York area network by using Transyt and Synchro with real facts and figures.

The methodology adopted for traffic operational analysis by use of software is as follows: At first, field measured data is fed to the network in the software package and performance of the existing network evaluated, this is referred to as base condition. The signal offset optimization is then performed to improve the current level of service. To elaborate the procedure; first the lane configurations are input, then traffic volumes, followed by signal timing and phase configuration of each intersection.

Simulation for the network resulted in errors because the volume data input in the software did not cater the traffic coming from unsignalized intersections in the network. Volume balancing was done for both routes of the network and simulation was run.

The file was then executed for optimization in order to optimize the splits and cycle lengths. The optimized parametric data was again fed into the software to get the modified or optimized system performance.

The next section would elaborate software analysis along with the results and recommended countermeasures. 


\subsection{Analysis}

A detailed analysis was performed with different strategies to enhance the LOS for the network for which traffic volume data was obtained from the City of Toronto, and signal timing plans, lane configurations, were determined from the field. This study is performed in two different stages. The first approach is to assess the existing condition of the network and problem identification. The second approach involves assessment of changes in the level of service using optimized signal splits and optimized cycle lengths followed by development of effective alternatives to resolve the problem.

\subsubsection{Transyt Analysis}

Base Case Assessment and Optimization Results for Transyt:

The base case evaluation showed O'Connor Avenue was the most poorly performing arterial with 2 signalized intersections at LOS F and 1 intersection at LOS E. The optimization improved the average delays to an extent with appreciable improvement only at Coxwell \& O'Connor where the LOS changed from $\mathrm{F}$ to $\mathrm{E}$. Two other failing intersections in the network remained at $\mathrm{F}$; however, the average delay times were improved.

\begin{tabular}{|l|l|c|c|c|c|c|c|c|c|}
\hline No & \multicolumn{1}{|c|}{ Name } & \multicolumn{2}{c|}{$\begin{array}{c}\text { Fuel } \\
\text { Consumption } \\
\text { (lit) }\end{array}$} & \multicolumn{2}{c|}{$\begin{array}{c}\text { Average } \\
\text { Delay } \\
\text { (sec/veh) }\end{array}$} & \multicolumn{2}{c|}{$\begin{array}{c}\text { Disutility } \\
\text { Index }\end{array}$} & \multicolumn{2}{c|}{$\begin{array}{c}\text { Level of } \\
\text { Service }\end{array}$} \\
\hline & & Sim. & Opt. & Sim. & Opt. & Sim. & Opt. & Sim. & Opt. \\
\hline 1 & Coxwell-Mortimer & 245 & 243 & 40 & 40 & 32 & 31 & D & D \\
\hline 2 & Coxwell-Cosburn & 100 & 100 & 13 & 12 & 9 & 9 & B & B \\
\hline 3 & Coxwell-Plains & 76 & 90 & 13 & 20 & 9 & 12 & B & C \\
\hline 4 & Coxwell-O'Connor & 607 & 508 & 94 & 75 & 102 & 74 & F & E \\
\hline 5 & O'Connor-Don Mills & 484 & 484 & 68 & 69 & 79 & 79 & E & E \\
\hline 6 & O'Connor-Donlands & 1001 & 905 & 348 & 314 & 245 & 219 & F & F \\
\hline 7 & Donlands-Cosburn & 144 & 143 & 14 & 14 & 12 & 12 & B & B \\
\hline 8 & Cosburn-Greenwood & 100 & 98 & 20 & 19 & 11 & 11 & C & B \\
\hline 9 & Greenwood-Mortimer & 228 & 239 & 84 & 92 & 42 & 45 & F & F \\
\hline
\end{tabular}

Table 3: Transyt Results- Network Performance Summary

Table above shows Transyt simulation and split optimization results which lead us to development of rectification techniques. As seen from the results, split optimization alone does little to improve level of service so I shall now take up individual intersections case by case and try various options to reduce delays and better level of service. 


\section{Coxwell \& Mortimer}

Coxwell \& Mortimer was node 1 of the network and had level of service D which is generally acceptable. Eastbound through traffic had greater delays due to higher traffic volumes and had an LOS of $\mathrm{E}$ but overall intersection was seen to function smoothly.

\begin{tabular}{|c|c|c|c|c|c|c|c|c|c|c|c|c|}
\hline \multirow[b]{2}{*}{ NODE 1} & \multicolumn{3}{|c|}{$\overline{\mathbf{E B}}$} & \multicolumn{3}{|c|}{ WB } & \multicolumn{3}{|c|}{ NB } & \multicolumn{3}{|c|}{ SB } \\
\hline & $\mathbf{L}$ & $\overline{\mathbf{T}}$ & $\mathbf{R}$ & $\mathbf{L}$ & $\mathbf{T}$ & $\mathbf{R}$ & $\mathbf{L}$ & $\mathbf{T}$ & $\mathbf{R}$ & $\mathbf{L}$ & $\mathbf{T}$ & $\mathbf{R}$ \\
\hline \multirow{6}{*}{$\begin{array}{l}\mathrm{V}(\mathrm{vph}) \\
\text { Saturation }(\%) \\
\text { Avg. Delay }(\mathrm{s} / \mathrm{v}) \\
\text { Fuel consumption }(\mathrm{L}) \\
\text { Effective green }(\mathrm{s}) \\
\text { Level of Service }\end{array}$} & & 856 & 107 & 32 & 274 & & & 591 & & 73 & 437 & \\
\hline & & 107 & 12 & 40 & 27 & & & 46 & & 32 & 67 & \\
\hline & & 64 & 17 & 33 & 12 & & & 22 & & 46 & 41 & \\
\hline & & 120 & 10 & 2 & 16 & & & 41 & & 7 & 45 & \\
\hline & & 55 & 55 & 55 & 55 & & & 35 & & 35 & 35 & \\
\hline & & $\mathrm{E}$ & $\bar{B}$ & $\mathrm{C}$ & $\bar{B}$ & & & $\mathrm{C}$ & & $\mathrm{D}$ & $\mathrm{D}$ & \\
\hline & & $\overline{\mathrm{RA}}$ & 7 & & $\mathrm{EC}$ & & & $\bar{S}$ & & & & \\
\hline
\end{tabular}

Table 4: Base Case Analysis Results- Coxwell \& Mortimer

\begin{tabular}{|c|c|c|c|c|c|c|c|c|c|c|c|c|c|}
\hline \multirow[b]{2}{*}{ NODE 1} & \multicolumn{3}{|c|}{ EB } & \multicolumn{3}{|c|}{ WB } & \multicolumn{3}{|c|}{ NB } & \multicolumn{3}{|c|}{$\overline{\text { SB }}$} & \multirow[t]{2}{*}{ INTERS. } \\
\hline & $\mathbf{L}$ & $\mathbf{T}$ & $\mathbf{R}$ & $\mathbf{L}$ & $T$ & $\underline{\mathbf{R}}$ & $\mathbf{L}$ & $\mathrm{T}$ & $\mathbf{R}$ & L & $\mathbf{T}$ & $\mathbf{R}$ & \\
\hline \multirow{4}{*}{$\begin{array}{l}\text { Initial delay }(\mathrm{s} / \mathrm{v}) \\
\text { Final delay }(\mathrm{s} / \mathrm{v}) \\
\text { Initial LOS } \\
\text { Final LOS }\end{array}$} & & 64 & 17 & 33 & 12 & & & 22 & & 46 & 41 & & 40 \\
\hline & & 61 & 17 & 30 & 12 & & & 22 & & 49. & 45 & & 40 \\
\hline & & $E$ & $\mathrm{~B}$ & $\mathrm{C}$ & $\mathrm{B}$ & & & $\mathrm{C}$ & & $\mathrm{D}$ & $\mathrm{D}$ & & D \\
\hline & & $E$ & $\mathrm{~B}$ & $\mathrm{C}$ & B & & & $\mathrm{C}$ & & $\mathrm{D}$ & $\mathrm{D}$ & & $\mathrm{D}$ \\
\hline
\end{tabular}

Table 5: Results after Split Optimization- Coxwell \& Mortimer

\begin{tabular}{|c|c|c|c|c|c|c|c|c|c|c|c|c|c|}
\hline \multirow[b]{2}{*}{ NODE 1} & \multicolumn{3}{|c|}{ EB } & \multicolumn{3}{|c|}{ WB } & \multicolumn{3}{|c|}{ NB } & \multicolumn{3}{|c|}{ SB } & \multirow{2}{*}{ INTERS. } \\
\hline & L & $\mathbf{T}$ & $\mathbf{R}$ & $\mathbf{L}$ & $T$ & $\mathbf{R}$ & $\mathbf{L}$ & $\mathbf{T}$ & $\mathbf{R}$ & $L$ & $\mathbf{T}$ & $\mathbf{R}$ & \\
\hline \multirow{4}{*}{$\begin{array}{l}\text { Initial delay }(\mathrm{s} / \mathrm{v}) \\
\text { Final delay }(\mathrm{s} / \mathrm{v}) \\
\text { Initial LOS } \\
\text { Final LOS } \\
\end{array}$} & & 64 & 17 & 33 & 12 & & & 22 & & 46 & 41 & & 40 \\
\hline & & 61 & 18 & 56 & 15 & & & 29 & & 43 & 48 & & 43 \\
\hline & & $E$ & $\mathrm{~B}$ & $\mathrm{C}$ & B & & & C & & $\mathrm{D}$ & $\mathrm{D}$ & & $\mathrm{D}$ \\
\hline & & $\mathrm{E}$ & B & $E$ & B & & & $\mathrm{C}$ & & $\mathrm{D}$ & $\mathrm{D}$ & & D \\
\hline
\end{tabular}

Table 6: Results after Cycle Length Optimization- Coxwell \& Mortimer 


\section{Coxwell \& Cosburn}

Coxwell \& Cosburn was also observed to function efficiently with a level of service at B.

\begin{tabular}{|c|c|c|c|c|c|c|c|c|c|c|c|c|}
\hline \multirow[b]{2}{*}{ NODE 2} & \multicolumn{3}{|c|}{ EB } & \multicolumn{3}{|c|}{ WB } & \multicolumn{3}{|c|}{ NB } & \multicolumn{3}{|c|}{ SB } \\
\hline & $\mathbf{L}$ & $\mathbf{T}$ & $\mathbf{R}$ & $\mathbf{L}$ & $T$ & $\mathbf{R}$ & $\mathbf{L}$ & $\mathbf{T}$ & $\mathbf{R}$ & $\mathbf{L}$ & $\mathbf{T}$ & $\mathbf{R}$ \\
\hline \multirow{6}{*}{$\begin{array}{l}\mathrm{V}(\mathrm{vph}) \\
\text { Saturation }(\%) \\
\text { Avg. Delay }(\mathrm{s} / \mathrm{v}) \\
\text { Fuel consumption (L) } \\
\text { Effective green (s) } \\
\text { Level of Service }\end{array}$} & & 331 & & 40 & 253 & & & 651 & & & 587 & \\
\hline & & 45 & & 19 & 55 & & & 57 & & & 31 & \\
\hline & & 28 & & 36 & 37 & & & 4 & & & 3 & \\
\hline & & 25 & & 3 & 21 & & & 30 & & & 19 & \\
\hline & & 25 & & 25 & 25 & & & 65 & & & 65 & \\
\hline & & $\mathrm{C}$ & & $\mathrm{D}$ & $\mathrm{D}$ & & & $\mathrm{A}$ & & & $\mathrm{A}$ & \\
\hline
\end{tabular}

Table 7: Base Case Analysis Results- Coxwell \& Cosburn

\begin{tabular}{|c|c|c|c|c|c|c|c|c|c|c|c|c|c|}
\hline \multirow[b]{2}{*}{ NODE 2} & \multicolumn{3}{|c|}{ EB } & \multicolumn{3}{|c|}{ WB } & \multicolumn{3}{|c|}{ NB } & \multicolumn{3}{|c|}{ SB } & \multirow[t]{2}{*}{\begin{tabular}{|l|} 
INTERS. \\
\end{tabular}} \\
\hline & L & $\mathbf{T}$ & $\mathbf{R}$ & L & $\mathbf{T}$ & $\mathbf{R}$ & $\mathbf{L}$ & $\mathbf{T}$ & $\mathbf{R}$ & L & $\mathbf{T}$ & $\mathbf{R}$ & \\
\hline \multirow{4}{*}{$\begin{array}{l}\text { Initial delay }(\mathrm{s} / \mathrm{v}) \\
\text { Final delay }(\mathrm{s} / \mathrm{v}) \\
\text { Initial LOS } \\
\text { Final LOS }\end{array}$} & & 28 & & 36 & 37 & & & 4 & & & 3 & & 13 \\
\hline & & 17 & & 21 & 22 & & & 13 & & & 4 & & 12 \\
\hline & & $\mathrm{C}$ & & D & $\mathrm{D}$ & & & $\mathrm{A}$ & & & $\mathrm{A}$ & & $\mathrm{B}$ \\
\hline & & $\mathrm{B}$ & & $\mathrm{C}$ & $\mathrm{C}$ & & & $\mathrm{B}$ & & & $\mathrm{A}$ & & $\mathrm{B}$ \\
\hline
\end{tabular}

Table 8: Results after Split Optimization- Coxwell \& Cosburn

\begin{tabular}{|c|c|c|c|c|c|c|c|c|c|c|c|c|c|}
\hline \multirow[b]{2}{*}{ NODE 2} & \multicolumn{3}{|c|}{ EB } & \multicolumn{3}{|c|}{ WB } & \multicolumn{3}{|c|}{ NB } & \multicolumn{3}{|c|}{ SB } & \multirow[t]{2}{*}{ INTERS. } \\
\hline & $\bar{L}$ & $T$ & $\overline{\mathbf{R}}$ & $\mathbf{L}$ & $T$ & $\overline{\mathbf{R}}$ & $\mathbf{L}$ & $\mathbf{T}$ & $\overline{\mathbf{R}}$ & $\mathbf{L}$ & $\mathbf{T}$ & $\overline{\mathbf{R}}$ & \\
\hline \multirow{4}{*}{$\begin{array}{l}\text { Initial delay }(\mathrm{s} / \mathrm{v}) \\
\text { Final delay }(\mathrm{s} / \mathrm{v}) \\
\text { Initial LOS } \\
\text { Final LOS }\end{array}$} & & 28 & & 36 & 37 & - & & 4 & & & 3 & & 13 \\
\hline & & 37 & & 46 & 45 & & & 5 & & & 4 & & 15 \\
\hline & & C & & $\mathrm{D}$ & $\mathrm{D}$ & & & $\bar{A}$ & & & $\bar{A}$ & & $\mathrm{~B}$ \\
\hline & & D & & $\mathrm{D}$ & $\mathrm{D}$ & & & A & & & $\bar{A}$ & & B \\
\hline
\end{tabular}

Table 9: Results after Cycle Length Optimization- Coxwell \& Cosburn 


\section{Coxwell \& Plains}

Coxwell \& Plains had an LOS of B which means intersection is running smoothly with minima delays. Optimization done in Transyt actually worsens the LOS from B to $\mathrm{C}$ as delays are seen to increase. Overall the results are acceptable and no corrective action needs to be implemented.

\begin{tabular}{|c|c|c|c|c|c|c|c|c|c|c|c|c|}
\hline \multirow[b]{2}{*}{ NODE 3} & \multicolumn{3}{|c|}{ EB } & \multicolumn{3}{|c|}{ WB } & \multicolumn{3}{|c|}{ NB } & \multicolumn{3}{|c|}{ SB } \\
\hline & $\mathbf{L}$ & $\mathbf{T}$ & $\mathbf{R}$ & $\mathbf{L}$ & $T$ & $\overline{\mathbf{R}}$ & $\mathbf{L}$ & $\mathbf{T}$ & $\mathbf{R}$ & $\mathbf{L}$ & $\mathbf{T}$ & $\mathbf{R}$ \\
\hline \multirow{6}{*}{$\begin{array}{l}\text { V (vph) } \\
\text { Saturation (\%) } \\
\text { Avg. Delay (s/v) } \\
\text { Fuel consumption (L) } \\
\text { Effective green (s) } \\
\text { Level of Service }\end{array}$} & & 251 & & & 164 & & & 650 & & & 470 & \\
\hline & & 59 & & & 42 & & & 41 & & & 22 & \\
\hline & & 27 & & & 22 & & & 10 & & & 6 & \\
\hline & & 18 & & & 11 & & & 23 & & & 22 & \\
\hline & & 36 & & & 36 & & & 54 & & & 54 & \\
\hline & & $\mathrm{C}$ & & & $\mathrm{C}$ & & & B &. & & $\bar{A}$ & \\
\hline \multicolumn{13}{|c|}{ OVERALL INTERSECTION: LOS B } \\
\hline
\end{tabular}

Table 10: Base Case Analysis Results - Coxwell \& Plains

\begin{tabular}{|c|c|c|c|c|c|c|c|c|c|c|c|c|c|}
\hline \multirow[b]{2}{*}{ NODE 3} & \multicolumn{3}{|c|}{ EB } & \multicolumn{3}{|c|}{ WB } & \multicolumn{3}{|c|}{ NB } & \multicolumn{3}{|c|}{ SB } & \multirow[t]{2}{*}{ INTERS. } \\
\hline & $\mathbf{L}$ & $\mathbf{T}$ & $\mathbf{R}$ & $\mathbf{L}$ & $T$ & $\mathbf{R}$ & $\mathbf{L}$ & $\mathrm{T}$ & $\mathbf{R}$ & $\mathbf{L}$ & $\mathbf{T}$ & $\mathbf{R}$ & \\
\hline \multirow{4}{*}{$\begin{array}{l}\text { Initial delay }(\mathrm{s} / \mathrm{v}) \\
\text { Final delay }(\mathrm{s} / \mathrm{v}) \\
\text { Initial LOS } \\
\text { Final LOS } \\
\end{array}$} & & 27 & & & 22 & & & 10 & & & 6 & & 13 \\
\hline & & 27 & & & 22 & & & 27 & & & 7 & & 20 \\
\hline & & $\mathrm{C}$ & & & $\mathrm{C}$ & & & B & & & $\mathrm{A}$ & & $\mathrm{B}$ \\
\hline & & $\mathrm{C}$ & & & C & & & C & & & A & & C \\
\hline
\end{tabular}

Table 11: Results after Split Optimization- Coxwell \& Plains

\begin{tabular}{|c|c|c|c|c|c|c|c|c|c|c|c|c|c|}
\hline \multirow[b]{2}{*}{ NODE 3} & \multicolumn{3}{|c|}{$\overline{E B}$} & \multicolumn{3}{|c|}{ WB } & \multicolumn{3}{|c|}{ NB } & \multicolumn{3}{|c|}{ SB } & \multirow[t]{2}{*}{ INTERS. } \\
\hline & $L$ & $T$ & $\mathbf{R}$ & $L$ & $\bar{T}$ & $\mathbf{R}$ & L & $T$ & $\mathbf{R}$ & $\mathbf{L}$ & $\mathbf{T}$ & $\overline{\mathbf{R}}$ & \\
\hline \multirow{4}{*}{$\begin{array}{l}\text { Initial delay }(\mathrm{s} / \mathrm{v}) \\
\text { Final delay }(\mathrm{s} / \mathrm{v}) \\
\text { Initial LOS } \\
\text { Final LOS }\end{array}$} & & 27 & & & 22 & & & 10 & & & 6 & & 13 \\
\hline & & 32 & & & 28 & & & 37 & & & 7 & & 25 \\
\hline & & C & & & C & & & B & & & $\mathrm{A}$ & & B \\
\hline & & $\mathrm{C}$ & & & $\bar{C}$ & & & $\mathrm{D}$ & & & $\mathrm{A}$ & & $\mathrm{C}$ \\
\hline
\end{tabular}

Table 12: Results after Cycle Length Optimization- Coxwell \& Plains 


\section{Coxwell \& O' Connor}

Coxwell and O'Connor is an intersection that needs immediate attention. This intersection experiences large delays and has large number of crashes as well. The cause of the problem at this intersection is excessive eastbound through traffic which results in an LOS of F. Optimization in Transyt reduced average delays in individual directions and improved LOS of the intersection from $\mathrm{F}$ to $\mathrm{E}$.

\begin{tabular}{|c|c|c|c|c|c|c|c|c|c|c|c|}
\hline \multirow[b]{2}{*}{ NODE 4} & \multicolumn{2}{|l|}{ EB } & \multicolumn{3}{|c|}{ WB } & \multicolumn{3}{|c|}{ NB } & \multicolumn{3}{|c|}{ SB } \\
\hline & $\mathbf{T}$ & $\mathbf{R}$ & $\mathbf{L}$ & $\mathbf{T}$ & $\mathbf{R}$ & $\mathbf{L}$ & $\mathbf{T}$ & $\mathbf{R}$ & $\mathbf{L}$ & $\mathbf{T}$ & $\mathbf{R}$ \\
\hline \multirow{6}{*}{$\begin{array}{l}\text { (vph) } \\
\text { Saturation (\%) } \\
\text { Avg. Delay (s/v) } \\
\text { Fuel consumption (L) } \\
\text { Effective green (s) } \\
\text { Level of Service }\end{array}$} & 2176 & & & 1067 & & & 611 & & & 154 & \\
\hline & 85 & & & 94 & & & 196 & & & 29 & \\
\hline & 12 & & & 21 & & & 530 & & & 32 & \\
\hline & 249 & & & 68 & & & 276 & & & 12 & \\
\hline & 63 & & & 73 & & & 17 & & & 17 & \\
\hline & B & & & $\mathrm{C}$ & & & $\mathrm{F}$ & & & $\mathrm{C}$ & \\
\hline
\end{tabular}

Table 13: Base Case Analysis Results- Coxwell \& O' Connor

\begin{tabular}{|c|c|c|c|c|c|c|c|c|c|c|c|c|c|}
\hline \multirow[b]{2}{*}{ NODE 4} & \multicolumn{3}{|c|}{ EB } & \multicolumn{3}{|c|}{ WB } & \multicolumn{3}{|c|}{ NB } & \multicolumn{3}{|c|}{$\overline{\text { SB }}$} & \multirow[t]{2}{*}{ INTERS. } \\
\hline & $\mathbf{L}$ & $T$ & $\overline{\mathbf{R}}$ & $\mathbf{L}$ & $\mathbf{T}$ & $\overline{\mathbf{R}}$ & $\mathbf{L}$ & $\mathbf{T}$ & $\overline{\mathbf{R}}$ & $\mathbf{L}$ & $T$ & $\mathbf{R}$ & \\
\hline Initial delay $(\mathrm{s} / \mathrm{v})$ & & 12 & & & 21 & & & 530 & & & 32 & & 94 \\
\hline Final delay $(\mathrm{s} / \mathrm{v})$ & & 5 & & & 8 & & & 691 & & & 44 & & 75 \\
\hline Initial LOS & & $\bar{B}$ & & & $\mathrm{C}$ & & & $\bar{F}$ & & & $\mathrm{C}$ & & $\bar{F}$ \\
\hline Final LOS & & $\bar{A}$ & & & $\bar{A}$ & & & $F$ & & & $\mathrm{D}$ & & $\bar{E}$ \\
\hline
\end{tabular}

Table 14: Results after Split Optimization- Coxwell \& O' Connor

\begin{tabular}{|c|c|c|c|c|c|c|c|c|c|c|c|c|c|}
\hline \multirow[b]{2}{*}{ NODE 4} & \multicolumn{3}{|c|}{ EB } & \multicolumn{3}{|c|}{ WB } & \multicolumn{3}{|c|}{ NB } & \multicolumn{3}{|c|}{ SB } & \multirow[t]{2}{*}{ INTERS. } \\
\hline & $\mathbf{L}$ & $\mathbf{T}$ & $\overline{\mathbf{R}}$ & $\mathbf{L}$ & $T$ & $\mathbf{R}$ & I & $\mathbf{T}$ & $\mathbf{R}$ & $\mathbf{L}$ & $\mathbf{T}$ & $\mathbf{R}$ & \\
\hline \multirow{4}{*}{$\begin{array}{l}\text { Initial delay }(\mathrm{s} / \mathrm{v}) \\
\text { Final delay }(\mathrm{s} / \mathrm{v}) \\
\text { Initial LOS } \\
\text { Final LOS }\end{array}$} & & 12 & & & 21 & & & 530 & & & 32 & & 94 \\
\hline & & 25 & & & 24 & & & 314 & & & 42 & & 59 \\
\hline & & $\bar{B}$ & & & $\mathrm{C}$ & & & $F$ & & & $\mathrm{C}$ & & $\bar{F}$ \\
\hline & & $\mathrm{C}$ & & & $\bar{C}$ & & & $F$ & & & $\mathrm{D}$ & & $\mathrm{E}$ \\
\hline
\end{tabular}

Table 15: Results after Cycle Length Optimization- Coxwell \& O' Connor 
Since optimization for splits and cycle length did not help reduce delays significantly, alteration in intersection geometry and lane configuration was resorted to. Options tried were introducing turn bay storage of 8 vehicles to the right turn lane in northbound direction and making northbound left turn exclusive. These directions had excessive traffic which caused congestion at the intersection. These changes made significant improvement in LOS and brought down intersection average delay from 94 seconds per vehicle to 36 seconds per vehicle. LOS achieved after adoption of countermeasures was $D$.

\begin{tabular}{|l|c|l|c|}
\hline \multicolumn{2}{|l|}{} & \multicolumn{2}{l}{} \\
\hline Overall Intersection Results for Simulation & Overall Intersection Results after changes \\
\hline Output Flow (vph) & 4008 & Output Flow (vph) & 3877 \\
\hline Degree of Sat. (\%) & 196 & Degree of Sat. (\%) & 102 \\
\hline Avg. Delay (sec/v) & 94 & Avg. Delay (sec/v) & 36 \\
\hline Fuel Consumpt. (lit) & 607 & Fuel Consumpt. (lit) & $421^{\prime}$ \\
\hline Disutility Index & 102 & Disutility Index & 48 \\
\hline Level of Service & F & Level of Service & D \\
\hline
\end{tabular}

Table 16: Results after changes in lane configuration- Coxwell \& $O$ ' Connor

Effect of these changes bettered LOS for intersection 4; however, it had adverse effect on the adjacent intersection. Intersection 3- Coxwell and Plains's LOS suffered and became D from an initial LOS of B. The fuel consumption increased manifold and became 134 liters from an original 76 liters which is unfortunate from environmental viewpoint. 


\section{O'Connor \& Don Mills}

Simulation results show an LOS of E for the intersection which is generally acceptable. Optimization of existing conditions was done but it did not help reduce delays for the intersection. To improve LOS, intersection parameters were changed and input in Transyt. Factors were considered individually to determine the effect of each in improving the quality of traffic flow. Alterations made were addition of a hypothetical lane in eastbound direction and addition of protected left turning phase. Signal splits were also changed but little was achieved to better the intersection LOS despite all these alterations.

\begin{tabular}{|c|c|c|c|c|c|c|c|c|c|c|c|c|}
\hline \multirow[b]{2}{*}{ NODE 5} & \multicolumn{3}{|c|}{ EB } & \multicolumn{3}{|c|}{ WB } & \multicolumn{3}{|c|}{ NB } & \multicolumn{3}{|c|}{ SB } \\
\hline & $\mathbf{L}$ & $\mathbf{T}$ & $\mathbf{R}$ & $\mathbf{L}$ & $\mathbf{T}$ & $\mathbf{R}$ & $\mathbf{L}$ & $\mathbf{T}$ & $\mathbf{R}$ & $\mathbf{L}$ & $\mathbf{T}$ & $\mathbf{R}$ \\
\hline \multirow{6}{*}{$\begin{array}{l}\mathrm{V}(\mathrm{vph}) \\
\text { Saturation }(\%) \\
\text { Avg. Delay }(\mathrm{s} / \mathrm{v}) \\
\text { Fuel consumption (L) } \\
\text { Effective green (s) } \\
\text { Level of Service }\end{array}$} & 424 & 603 & & & 507 & 520 & & & & 1560 & & 394 \\
\hline & 100 & 49 & & & 137 & 38 & & & & 96 & & 45 \\
\hline & 105 & 31 & & & 278 & 2 & & & & 41 & & 14 \\
\hline & 53 & 48 & & & 171 & 45 & & & & 141 & & 24 \\
\hline & 45 & 35 & & & 35 & 85 & & & & 45 & & 57 \\
\hline & $\overline{\mathrm{F}}$ & $\bar{C}$ & & & $\mathrm{~F}$ & $\bar{A}$ & & & & $\bar{D}$ & & $\mathrm{~B}$ \\
\hline
\end{tabular}

Table 17: Base Case Analysis Results - O' Connor \& Don Mills

\begin{tabular}{|c|c|c|c|c|c|c|c|c|c|c|c|c|}
\hline \multirow[b]{2}{*}{ NODE 5} & \multicolumn{3}{|c|}{ EB } & \multicolumn{3}{|c|}{ WB } & \multicolumn{3}{|c|}{ NB } & \multicolumn{2}{|c|}{ SB } & \multirow[t]{2}{*}{ INTERS. } \\
\hline & $\mathbf{L}$ & $T$ & $\overline{\mathbf{R}}$ & $\mathbf{L}$ & $\mathbf{T}$ & $\mathbf{R}$ & $\mathbf{L}$ & $\mathbf{T}$ & $\mathbf{R}$ & & $\mathbf{R}$ & \\
\hline \multirow{4}{*}{$\begin{array}{l}\text { Initial delay }(\mathrm{s} / \mathrm{v}) \\
\text { Final delay }(\mathrm{s} / \mathrm{v}) \\
\text { Initial LOS } \\
\text { Final LOS }\end{array}$} & 105 & 31 & & & 278 & 2 & & & & 41 & 14 & 68 \\
\hline & 70 & 32 & & & 359 & 2 & & & & 46 & 13 & 69 \\
\hline & $F$ & $\mathrm{C}$ & & & $\bar{F}$ & $\mathrm{~A}$ & & & & $\mathrm{D}$ & $\mathrm{B}$ & $E$ \\
\hline & $\bar{E}$ & $\mathrm{C}$ & & & $\bar{F}$ & $\bar{A}$ & & & & $\bar{D}$ & $\bar{B}$ & $\bar{E}$ \\
\hline
\end{tabular}

Table 18: Results after Split Optimization- $O^{\prime}$ Connor \& Don Mills

\begin{tabular}{|c|c|c|c|c|c|c|c|c|c|c|c|c|c|}
\hline \multirow[b]{2}{*}{ NODE 5} & \multicolumn{3}{|c|}{ EB } & \multicolumn{3}{|c|}{ WB } & \multicolumn{3}{|c|}{ NB } & \multicolumn{3}{|c|}{ SB } & \multirow[t]{2}{*}{ INTERS. } \\
\hline & $\mathbf{L}$ & $T$ & $\overline{\mathbf{R}}$ & $\mathbf{L}$ & $\mathbf{T}$ & $\mathbf{R}$ & $\mathbf{L}$ & $\mathbf{T}$ & $\mathbf{R}$ & & $\mathbf{T}$ & $\mathbf{R}$ & \\
\hline \multirow{4}{*}{$\begin{array}{l}\text { Initial delay }(\mathrm{s} / \mathrm{v}) \\
\text { Final delay }(\mathrm{s} / \mathrm{v}) \\
\text { Initial LOS } \\
\text { Final LOS }\end{array}$} & 105 & 31 & & & 278 & 2 & & & & 41 & & 14 & 68 \\
\hline & 101 & 43 & & & 295 & 1 & & & & 39 & & $\overline{86}$ & 78 \\
\hline & $\mathrm{F}$ & $\mathrm{C}$ & & & $\bar{F}$ & $\overline{\mathrm{A}}$ & & & & $\mathrm{D}$ & & B & $\mathrm{E}$ \\
\hline & $F$ & $\mathrm{D}$ & & & $\bar{F}$ & $\bar{A}$ & & & & D & & $\bar{F}$ & $\mathrm{E}$ \\
\hline
\end{tabular}

Table 19: Results after Cycle Length Optimization- O' Connor \& Don Mills 


\section{O' Connor \& Donlands}

Simulation results show that the intersection is failing as level of service is F. Even with optimization in Transyt, little improvement is seen in reduction of delays and fuel consumption.

\begin{tabular}{|c|c|c|c|c|c|c|c|c|c|c|c|c|}
\hline \multirow[b]{2}{*}{ NODE 6} & \multicolumn{3}{|c|}{ EB } & \multicolumn{3}{|c|}{ WB } & \multicolumn{3}{|c|}{$\widehat{N B}$} & \multicolumn{3}{|c|}{$\overline{\text { SB }}$} \\
\hline & $\bar{L}$ & $\mathbf{T}$ & $\overline{\mathbf{R}}$ & $\bar{L}$ & $T$ & $\overline{\mathbf{R}}$ & $\mathbf{L}$ & $T$ & $\mathbf{R}$ & $\bar{L}$ & $T$ & $\overline{\mathbf{R}}$ \\
\hline \multirow{6}{*}{$\begin{array}{l}\text { V (vph) } \\
\text { Saturation (\%) } \\
\text { Avg. Delay }(\mathrm{s} / \mathrm{v}) \\
\text { Fuel consumption (L) } \\
\text { Effective green (s) } \\
\text { Level of Service }\end{array}$} & & 590 & 44 & & 626 & 48 & & 770 & & & 949 & 20 \\
\hline & & 85 & 8 & & 194 & 7 & & 52 & & & 232 & 3 \\
\hline & & 40 & 23 & & 574 & 44 & & 16 & & & 698 & 14 \\
\hline & & 52 & 3 & & 315 & 8 & & 53 & & & 567 & 1 \\
\hline & & 33 & 33 & & 43 & 43 & & 47 & & & 47 & 47 \\
\hline & & $\mathrm{D}$ & $\mathrm{C}$ & & $\bar{F}$ & $\mathrm{D}$ & & $\mathrm{B}$ & & & $F$ & B \\
\hline \multicolumn{13}{|c|}{ OVERALL INTERSECTION: LOS F } \\
\hline
\end{tabular}

Table 20: Base Case Analysis Results - O' Connor'\& Donlands

\begin{tabular}{|c|c|c|c|c|c|c|c|c|c|c|c|c|}
\hline \multirow[b]{2}{*}{ NODE 6} & \multicolumn{3}{|c|}{$\overline{E B}$} & \multicolumn{3}{|c|}{ WB } & \multicolumn{3}{|c|}{ NB } & \multicolumn{2}{|l|}{ SB } & \multirow{2}{*}{ INTERS. } \\
\hline & $\mathbf{L}$ & $T$ & $\mathbf{R}$ & $\mathbf{L}$ & $\mathbf{T}$ & $\mathbf{R}$ & $\mathbf{L}$ & $T$ & $\mathbf{R}$ & L. & $\mathbf{R}$ & \\
\hline \multirow{4}{*}{$\begin{array}{l}\text { Initial delay }(\mathrm{s} / \mathrm{v}) \\
\text { Final delay }(\mathrm{s} / \mathrm{v}) \\
\text { Initial LOS } \\
\text { Final LOS }\end{array}$} & & 40 & 23 & & 574 & 44 & & 16 & & 698 & 14 & 348 \\
\hline & & 44 & 24 & & 463 & 45 & & 15 & & 672 & 13 & 314 \\
\hline & & $\mathrm{D}$ & $\mathrm{C}$ & & $\mathrm{F}$ & $\mathrm{D}$ & & $\mathrm{B}$ & & $\mathrm{F}$ & $\mathrm{B}$ & $F$ \\
\hline & & $\bar{D}$ & $\mathrm{C}$ & & $\bar{F}$ & $\mathrm{D}$ & & $\bar{B}$ & & $\bar{F}$ & B & $F$ \\
\hline
\end{tabular}

Table 21: Results after Split Optimization- $O$ ' Connor \& Donlands

\begin{tabular}{|c|c|c|c|c|c|c|c|c|c|c|c|c|c|}
\hline \multirow[b]{2}{*}{ NODE 6} & \multicolumn{3}{|c|}{ EB } & \multicolumn{3}{|c|}{ WB } & \multicolumn{3}{|c|}{ NB } & \multicolumn{3}{|c|}{ SB } & \multirow[t]{2}{*}{ INTERS. } \\
\hline & $\mathbf{L}$ & $\mathbf{T}$ & $\mathbf{R}$ & $\mathbf{L}$ & $\mathbf{T}$ & $\mathbf{R}$ & $\mathbf{L}$ & $T$ & $\mathbf{R}$ & $\mathbf{L}$ & $T$ & $\mathbf{R}$ & \\
\hline \multirow{4}{*}{$\begin{array}{l}\text { Initial delay }(\mathrm{s} / \mathrm{v}) \\
\text { Final delay }(\mathrm{s} / \mathrm{v}) \\
\text { Initial LOS } \\
\text { Final LOS }\end{array}$} & & $\overline{40}$ & 23 & & 574 & $\overline{44}$ & & 16 & & & 698 & 14 & 348 \\
\hline & & 43 & 27 & & 494 & 54 & & 22 & & & 717 & 17 & $331^{\circ}$ \\
\hline & & D & C & & $F$ & D & & B & & & F & B & $F$ \\
\hline & & $\mathrm{D}$ & $\mathrm{C}$ & & $\mathrm{F}$ & D & & $\mathrm{C}$ & & & F & B & $\bar{F}$ \\
\hline
\end{tabular}

Table 22: Results after Cycle Length Optimization- $O$ ' Connor \& Donlands 
To alleviate the traffic situation, variety of lane configurations were input and simulation was run for O'Connor and Donlands. Addition of $6 \& 4$ turn bay storage for left \& right turn respectively for southbound traffic and addition of left turn bay storage of 8 vehicles for westbound traffic was done. No changes were made to phasing or signal timings. The results improved LOS as it changed from an initial $F$ to $D$.

\begin{tabular}{|l|c|l|c|}
\hline \multicolumn{2}{|l|}{} & \multicolumn{2}{l|}{} \\
\hline Overall Intersection Results for Simulation & Overall Intersection Results after changes \\
\hline Degree of Sat. (\%) & 3047 & Output Flow (vph) & 2453 \\
\hline Avg. Delay (sec/v) & 232 & Degree of Sat. (\%) & 116 \\
\hline Fuel Consumpt. (lit) & 348 & Avg. Delay (sec/v) & 54 \\
\hline Disutility Index & 1001 & Fuel Consumpt. (lit) & 238 \\
\hline Level of Service & 245 & Disutility Index & 41 \\
\hline
\end{tabular}

Table 23: Results after changes in lane configuration- $O$ ' Connor \& Donlands

Changes made to lane configurations at intersection 6 had favorable results for intersection 5 as well. The LOS at O' Connor \& Don Mills improved from E to D and fuel consumption reduced from an initial 484 liters to 406 liters. 


\section{Cosburn \& Donlands}

The intersection was seen to perform well under existing conditions. The level of service was B and traffic was moving well. This intersection had minimal crashes as well. The results for simulation and optimization have been shown below.

\begin{tabular}{|c|c|c|c|c|c|c|c|c|c|c|c|c|}
\hline \multirow[b]{2}{*}{ NODE 7} & \multicolumn{3}{|c|}{ EB } & \multicolumn{3}{|c|}{ WB } & \multicolumn{3}{|c|}{ NB } & \multicolumn{3}{|c|}{ SB } \\
\hline & $\mathbf{L}$ & $\mathbf{T}$ & $\mathbf{R}$ & $\mathbf{L}$ & $\bar{T}$ & $\mathbf{R}$ & $\mathbf{L}$ & $\mathbf{T}$ & $\mathbf{R}$ & $\mathbf{L}$ & $T$ & $\mathbf{R}$ \\
\hline \multirow{6}{*}{$\begin{array}{l}\text { V (vph) } \\
\text { Saturation }(\%) \\
\text { Avg. Delay }(\mathrm{s} / \mathrm{v}) \\
\text { Fuel consumption }(\mathrm{L}) \\
\text { Effective green }(\mathrm{s}) \\
\text { Level of Service }\end{array}$} & 42 & 349 & 49 & 68 & 337 & & & 708 & & & 486 & \\
\hline & 15 & 47 & 8 & 23 & 48 & & & 36 & & & 36 & \\
\hline & 25 & 24 & 19 & 14 & 16 & & & 12 & & & 7 & \\
\hline & 3 & 25 & 3 & 3 & 19 & & & 41 & & & 47 & \\
\hline & 39 & 39 & 39 & 39 & 39 & & & 51 & & & 51 & \\
\hline & $\mathrm{C}$ & $\mathrm{C}$ & $\mathrm{B}$ & $\bar{B}$ & $\bar{B}$ & & & $\bar{B}$ & & & $\bar{A}$ & \\
\hline \multicolumn{13}{|c|}{ OVERALL INTERSECTION: LOS B } \\
\hline
\end{tabular}

Table 24: Base Case Analysis Results- Cosburn \& Donlands

\begin{tabular}{|c|c|c|c|c|c|c|c|c|c|c|c|c|c|}
\hline \multirow[b]{2}{*}{ NODE 7} & \multicolumn{3}{|c|}{ EB } & \multicolumn{3}{|c|}{ WB } & \multicolumn{3}{|c|}{ NB } & \multicolumn{3}{|c|}{ SB } & \multirow[t]{2}{*}{ INTERS. } \\
\hline & $\mathbf{L}$ & $\mathbf{T}$ & $\mathbf{R}$ & $\mathbf{L}$ & $\mathbf{T}$ & $\mathbf{R}$ & $\mathbf{L}$ & $\mathbf{T}$ & $\mathbf{R}$ & & $\mathbf{T}$ & $\mathbf{R}$ & \\
\hline Initial delay $(\mathrm{s} / \mathrm{v})$ & 25 & 24 & 19 & 14 & 16 & & & 12 & & & 7 & & 14 \\
\hline Final delay $(\mathrm{s} / \mathrm{v})$ & 24 & 24 & 19 & 14 & 16 & & & 12 & & & 7 & & 14 \\
\hline Initial LOS & $\mathrm{C}$ & $\bar{C}$ & $\mathrm{~B}$ & $\mathrm{~B}$ & $\bar{B}$ & & & $\mathrm{~B}$ & & & $\bar{A}$ & & $\bar{B}$ \\
\hline Final LOS & C & C & B & B & B & & & B & & & A & & B \\
\hline
\end{tabular}

Table 25: Results after Split Optimization- Cosburn \& Donlands

\begin{tabular}{|c|c|c|c|c|c|c|c|c|c|c|c|c|c|}
\hline \multirow[b]{2}{*}{ NODE 7} & \multicolumn{3}{|c|}{ EB } & \multicolumn{3}{|c|}{ WB } & \multicolumn{3}{|c|}{ NB } & \multicolumn{3}{|c|}{ SB } & \multirow[t]{2}{*}{ INTERS. } \\
\hline & $\mathbf{L}$ & $\mathbf{T}$ & $\mathbf{R}$ & $\bar{L}$ & $\mathbf{T}$ & $\overline{\mathbf{R}}$ & $\mathbf{L}$ & $\mathbf{T}$ & $\mathbf{R}$ & $\mathbf{L}$ & $T$ & $\overline{\mathbf{R}}$ & \\
\hline Initial delay $(\mathrm{s} / \mathrm{v})$ & 25 & 24 & 19 & 14 & 16 & & & 12 & & & 7 & & 14 \\
\hline Final delay $(\mathrm{s} / \mathrm{v})$ & 38 & 31 & 25 & 18 & 21 & & & 15 & & & 16 & & 20 \\
\hline Initial LOS & $\mathrm{C}$ & $\mathrm{C}$ & B & B & B & & & B & & & A & & $\bar{B}$ \\
\hline Final LOS & $\mathrm{D}$ & $\mathrm{C}$ & $\mathrm{C}$ & B & C & & & B & & & $\bar{B}$ & & $\bar{C}$ \\
\hline
\end{tabular}

Table 26: Results after Cycle Length Optimization- Cosburn \& Donlands 


\section{Cosburn \& Greenwood}

Traffic at Cosburn and Greenwood was observed to be moving well. Overall intersection had an LOS of $\mathrm{C}$ which was improved to B after split optimization in Transyt.

\begin{tabular}{|c|c|c|c|c|c|c|c|c|c|c|c|c|}
\hline \multirow[b]{2}{*}{ NODE 8} & \multicolumn{3}{|c|}{ EB } & \multicolumn{3}{|c|}{ WB } & \multicolumn{3}{|c|}{ NB } & \multicolumn{3}{|c|}{$\overline{\text { SB }}$} \\
\hline & $\mathbf{L}$ & $T$ & $\bar{R}$ & $\mathbf{L}$ & $\mathbf{T}$ & $\mathbf{R}$ & $\mathbf{L}$ & $T$ & $\mathbf{R}$ & $\mathbf{L}$ & $\mathbf{T}$ & $\mathbf{R}$ \\
\hline \multirow{6}{*}{$\begin{array}{l}\mathrm{V}(\mathrm{vph}) \\
\text { Saturation }(\%) \\
\text { Avg. Delay }(\mathrm{s} / \mathrm{v}) \\
\text { Fuel consumption }(\mathrm{L}) \\
\text { Effective green }(\mathrm{s}) \\
\text { Level of Service }\end{array}$} & 33 & 489 & & 46 & 307 & & 99 & 133 & & & 396 & \\
\hline & 7 & 50 & & 14 & 31 & & 41 & 21 & & & 58 & \\
\hline & 11 & 15 & & 19 & 14 & & 36 & 28 & & & 24 & \\
\hline & 1 & 29 & & 3 & 18 & & 7 & 10 & & & 28 & \\
\hline & 52 & 52 & & 52 & 52 & & 38 & 38 & & & 38 & \\
\hline & $\mathrm{B}$ & $\mathrm{B}$ & & $\mathrm{B}$ & $\mathrm{B}$ & & $\mathrm{D}$ & $\mathrm{C}$ & & & $\mathrm{C}$ & \\
\hline
\end{tabular}

Table 27: Base Case Analysis Results- Cosburn \& Greenwood

\begin{tabular}{|c|c|c|c|c|c|c|c|c|c|c|c|c|c|}
\hline \multirow[b]{2}{*}{ NODE 8} & \multicolumn{3}{|c|}{ EB } & \multicolumn{3}{|c|}{ WB } & \multicolumn{3}{|c|}{ NB } & \multicolumn{3}{|c|}{ SB } & \multirow[t]{2}{*}{ INTERS. } \\
\hline & $\mathbf{L}$ & $\mathbf{T}$ & $\mathbf{R}$ & $\mathbf{L}$ & $\mathbf{T}$ & $\mathbf{R}$ & $\mathbf{L}$ & $\mathbf{T}$ & $\mathbf{R}$ & $\mathbf{L}$ & $T$ & $\mathbf{R}$ & \\
\hline Initial delay (s/v) & 11 & 15 & & 19 & 14 & & 36 & 28 & & & 24 & & 20 \\
\hline Final delay (s/v) & 13 & 20 & & 25 & 19 & & 19 & 18 & & & 18 & & 19 \\
\hline Initial LOS & B & B & & B & B & & D & C & & & C & & $\mathrm{C}$ \\
\hline Final LOS & B & $\mathrm{C}$ & & $\mathrm{C}$ & B & & B & $\mathrm{B}$ & & & $\mathrm{B}$ & & B \\
\hline
\end{tabular}

Table 28: Results after Split Optimization- Cosburn \& Greenwood

\begin{tabular}{|c|c|c|c|c|c|c|c|c|c|c|c|c|c|}
\hline \multirow[b]{2}{*}{ NODE 8} & \multicolumn{3}{|c|}{ EB } & \multicolumn{3}{|c|}{ WB } & \multicolumn{3}{|c|}{ NB } & \multicolumn{3}{|c|}{ SB } & \multirow[t]{2}{*}{ INTERS } \\
\hline & $\mathbf{L}$ & $\mathbf{T}$ & $\overline{\mathbf{R}}$ & $\mathbf{L}$ & $\mathbf{T}$ & $\overline{\mathbf{R}}$ & $\mathbf{L}$ & $\mathbf{T}$ & $\mathbf{R}$ & $\mathbf{L}$ & $\mathbf{T}$ & $\mathbf{R}$ & \\
\hline Initial delay (s/v) & 11 & 15 & & 19 & 14 & & 36 & 28 & & & 24 & & 20 \\
\hline Final delay (s/v) & 9 & 15 & & 22 & 17 & & 30 & 25 & & & 31 & & 22 \\
\hline Initial LOS & $\bar{B}$ & $\mathrm{~B}$ & & $\mathrm{~B}$ & $\mathrm{~B}$ & & D & $\mathrm{C}$ & & & $\mathrm{C}$ & & $\mathrm{C}$ \\
\hline Final LOS & $\mathrm{A}$ & $\mathrm{B}$ & & $\mathrm{C}$ & B & & $\mathrm{C}$ & $\mathrm{C}$ & & & $\mathrm{C}$ & & $\bar{C}$ \\
\hline
\end{tabular}

Table 29: Results after Cycle Length Optimization- Cosburn \& Greenwood 


\section{Mortimer \& Greenwood}

Traffic volumes were not very high but the intersection was seen to fail because only one lane catered for left, through and right turning movements. The Level of Service was acceptable in all directions but for southbound where it was failing. Changing signal timings did not improve the delays and thus LOS remained at $F$ even after optimization.

\begin{tabular}{|c|c|c|c|c|c|c|c|c|c|c|c|c|}
\hline \multirow[b]{2}{*}{ NODE 9} & \multicolumn{3}{|c|}{ EB } & \multicolumn{3}{|c|}{ WB } & \multicolumn{3}{|c|}{ NB } & \multicolumn{3}{|c|}{ SB } \\
\hline & $\mathbf{L}$ & $\mathbf{T}$ & $\mathbf{R}$ & $\mathbf{L}$ & $\mathbf{T}$ & $\mathbf{R}$ & $\mathbf{L}$ & $\mathbf{T}$ & $\mathbf{R}$ & $\mathbf{L}$ & $T$ & $\mathbf{R}$ \\
\hline \multirow{6}{*}{$\begin{array}{l}V(\mathrm{vph}) \\
\text { Saturation }(\%) \\
\text { Avg. Delay }(\mathrm{s} / \mathrm{v}) \\
\text { Fuel consumption }(\mathrm{L}) \\
\text { Effective green }(\mathrm{s}) \\
\text { Level of Service }\end{array}$} & & 753 & & & 316 & & & 374 & & & 391 & \\
\hline & & 69 & & & 31 & & & 68 & & & 159 & \\
\hline & & 14 & & & 8 & & & 32 & & & 331 & \\
\hline & & 46 & & & 28 & & & 30 & & & 123 & \\
\hline & & 59 & & & 59 & & & 31 & & & 31 & \\
\hline & & $\bar{B}$ & & & $\mathrm{~A}$ & & & $\mathrm{C}$ & & & $\bar{F}$ & \\
\hline \multicolumn{13}{|c|}{ OVERALL INTERSECTION: LOS F } \\
\hline
\end{tabular}

Table 30: Base Case Analysis Results - Mortimer \& Greenwood

\begin{tabular}{|c|c|c|c|c|c|c|c|c|c|c|c|c|c|}
\hline \multirow[b]{2}{*}{ NODE 9} & \multicolumn{3}{|c|}{ EB } & \multicolumn{3}{|c|}{ WB } & \multicolumn{3}{|c|}{ NB } & & \multicolumn{2}{|l|}{ SB } & \multirow[t]{2}{*}{ INTERS. } \\
\hline & $\bar{L}$ & $T$ & $\overline{\mathbf{R}}$ & $\overline{\mathbf{L}}$ & $\mathbf{T}$ & $\overline{\mathbf{R}}$ & $\bar{L}$ & $T$ & $\overline{\mathbf{R}}$ & & $\mathbf{T}$ & $\overline{\mathbf{R}}$ & \\
\hline \multirow{4}{*}{$\begin{array}{l}\text { Initial delay }(\mathrm{s} / \mathrm{v}) \\
\text { Final delay }(\mathrm{s} / \mathrm{v}) \\
\text { Initial LOS } \\
\text { Final LOS }\end{array}$} & & 14 & & & 8 & & & 32 & & & 331 & & $\overline{84}$ \\
\hline & & 13 & & & 8 & & & 34 & & & 364 & & 92 \\
\hline & & $\mathrm{B}$ & & & $\bar{A}$ & & & $\mathrm{C}$ & & & $\bar{F}$ & & $\bar{F}$ \\
\hline & & B & & & $\bar{A}$ & & & $\mathrm{C}$ & & & $\bar{F}$ & & $F$ \\
\hline
\end{tabular}

Table 31: Results after Split Optimization- Mortimer \& Greenwood

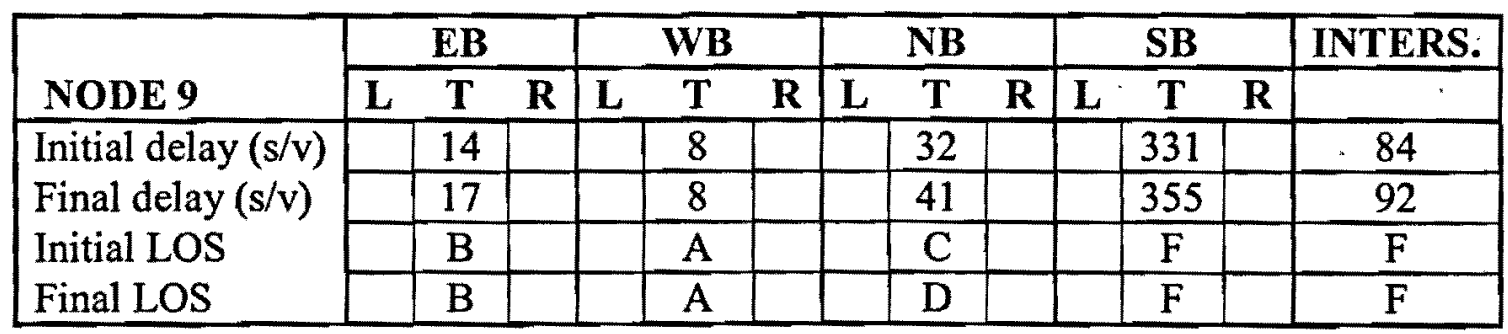

Table 32: Results after Cycle Length Optimization- Mortimer \& Greenwood 
Changes in lane configuration were made in an attempt to better LOS. An exclusive left turn was provided for southbound traffic which eased congestion in the intersection and improved level of service to D. Fuel consumption and delays reduced remarkably.

\begin{tabular}{|l|c|l|c|}
\hline \multicolumn{2}{|l|}{} & \multicolumn{2}{l|}{} \\
\hline & & & \\
\hline Output Flow (vph) & 1834 & Output Flow (vph) & 1836 \\
\hline Degree of Sat. (\%) & 159 & Degree of Sat. (\%) & 114 \\
\hline Avg. Delay (sec/v) & 84 & Avg. Delay (sec/v) & 38 \\
\hline Fuel Consumpt. (lit) & 228 & Fuel Consumpt. (lit) & 162 \\
\hline Disutility Index & 42 & Disutility Index & 22 \\
\hline Level of Service & F & Level of Service & D \\
\hline
\end{tabular}

Table 33: Results after changes in lane configuration- Mortimer \& Greenwood

No repercussions were observed at the adjacent intersections and level of service remained normal for intersection numbers 8 and 1 . 


\subsubsection{Synchro Analysis}

Synchro analysis shows similar results to the Transyt analysis. There are few differences in between the two packages as made clear by the figure.

\begin{tabular}{|c|c|c|c|c|c|c|c|c|c|c|}
\hline \multirow{2}{*}{ Prograx } & \multicolumn{2}{|l|}{. } & \multicolumn{3}{|c|}{ Aptiontoxs } & \multirow[b]{2}{*}{ 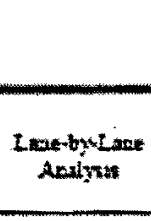 } & \multicolumn{2}{|c|}{ Arimatton } & \multicolumn{2}{|c|}{$\begin{array}{l}\text { Mexisures of } \\
\text { Effecnieness }\end{array}$} \\
\hline & $\begin{array}{l}\text { Evainat } \\
\text { Eximat } \\
\text { Tumane }\end{array}$ & $\begin{array}{c}\text { Opaniz: } \\
\text { Cyd: } \\
\text { Leapin }\end{array}$ & Openise & $\begin{array}{l}\text { Opodinise } \\
\text { splin }\end{array}$ & $\begin{array}{l}\text { Optinises } \\
\text { ECSLIOS }\end{array}$ & & Dypurate & seate & 105 & Connual \\
\hline TEENSYT-TH & $\sqrt{ }$ & $\sqrt{ }$ & $\checkmark$ & 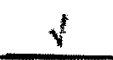 & No & $\sqrt{ }$ & $\mathrm{No}$ & $\sqrt{ }$ & $\sqrt{ }$ & $\sqrt{ }$ \\
\hline STIVHRO & $\sqrt{ }$ & $\sqrt{t}$ & $\sqrt{ }$ & $\sqrt{ }$ & $\forall$ & No & No & $\sqrt{ }$ & $\sqrt{ }$ & $\sqrt{ }$ \\
\hline corsm & $\sqrt{4}$ & No & No & No & No & $\mathrm{No}$ & $\sqrt{ }$ & $\mathrm{Na}$ & No & $\checkmark$ \\
\hline
\end{tabular}

Figure 4: Synchro \& Transyt comparison chart $^{(13)}$

\begin{tabular}{|c|l|c|c|c|}
\hline No. & \multicolumn{1}{|c|}{ Name } & $\begin{array}{c}\text { Intersection } \\
\text { Signal Delay } \\
\text { (secs) }\end{array}$ & $\begin{array}{c}\text { Intersection } \\
\text { Capacity Utilization } \\
\text { (\%) }\end{array}$ & $\begin{array}{c}\text { Intersection } \\
\text { LOS }\end{array}$ \\
\hline 1 & Coxwell-Mortimer & 255.9 & 114.8 & $\mathrm{~F}$ \\
\hline 2 & Coxwell-Cosburn & 17.5 & 98.3 & $\mathrm{~B}$ \\
\hline 3 & Coxwell-Plains & 13.8 & 69.5 & $\mathrm{~B}$ \\
\hline 4 & Coxwell-O'Connor & 323.5 & 135.1 a & $\mathrm{F}$ \\
\hline 5 & O'Connor-DonMills & 187 & 120.5 & $\mathrm{~F}$ \\
\hline 6 & O'Connor-Donlands & 688.5 & 167.2 & $\mathrm{~F}$ \\
\hline 7 & Donlands-Cosburn & 25.7 & 87.2 & $\mathrm{C}$ \\
\hline 8 & Cosburn-Greenwood & 21.3 & 78.2 & $\mathrm{C}$ \\
\hline 9 & Greenwood-Mortimer & 86.5 & 102.4 & $\mathrm{~F}$ \\
\hline
\end{tabular}

\section{Table 34: Synchro Results- Network Performance Summary}

Synchro implements Intersection Capacity Utilization method for determining intersection capacity. It compares current volumes to the intersection's maximum capacity. Intersection Capacity Utilization is similar to but not the same as intersection volume to capacity ratio. A value less than $100 \%$ indicates that the intersection has extra capacity. A value greater than $100 \%$ indicates the intersection is over capacity ${ }^{(12)} .5$ intersections in the network are seen to operate over $100 \%$ ICU. 
Measures of effectiveness in Synchro include delays, stops, fuel consumption and emissions and a variety of reports can be printed for analysis of simulation/optimization results. Majority of intersections in the network experience huge delays and are seen to have an LOS of F which is the lowest measurement of efficiency for a road's performance with every vehicle moving in lockstep with the vehicle in front of it.

The results in Transyt and Synchro analysis are similar and hence same treatment for the failing intersections is recommended. Note that ICU 2003 includes additional levels past F to further differentiate congested operation ${ }^{(12)}$.

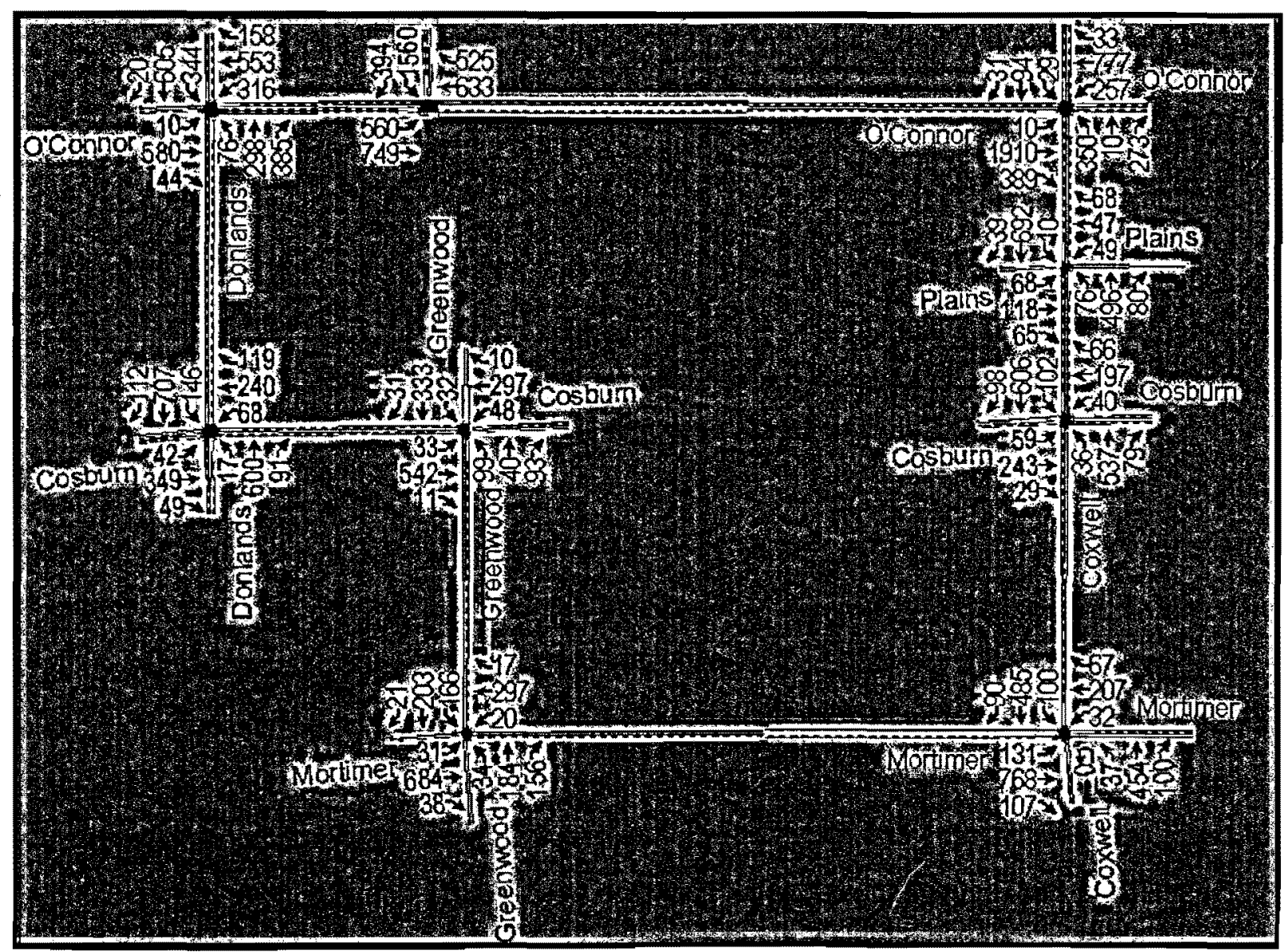

Figure 5: Synchro street network showing traffic volumes 


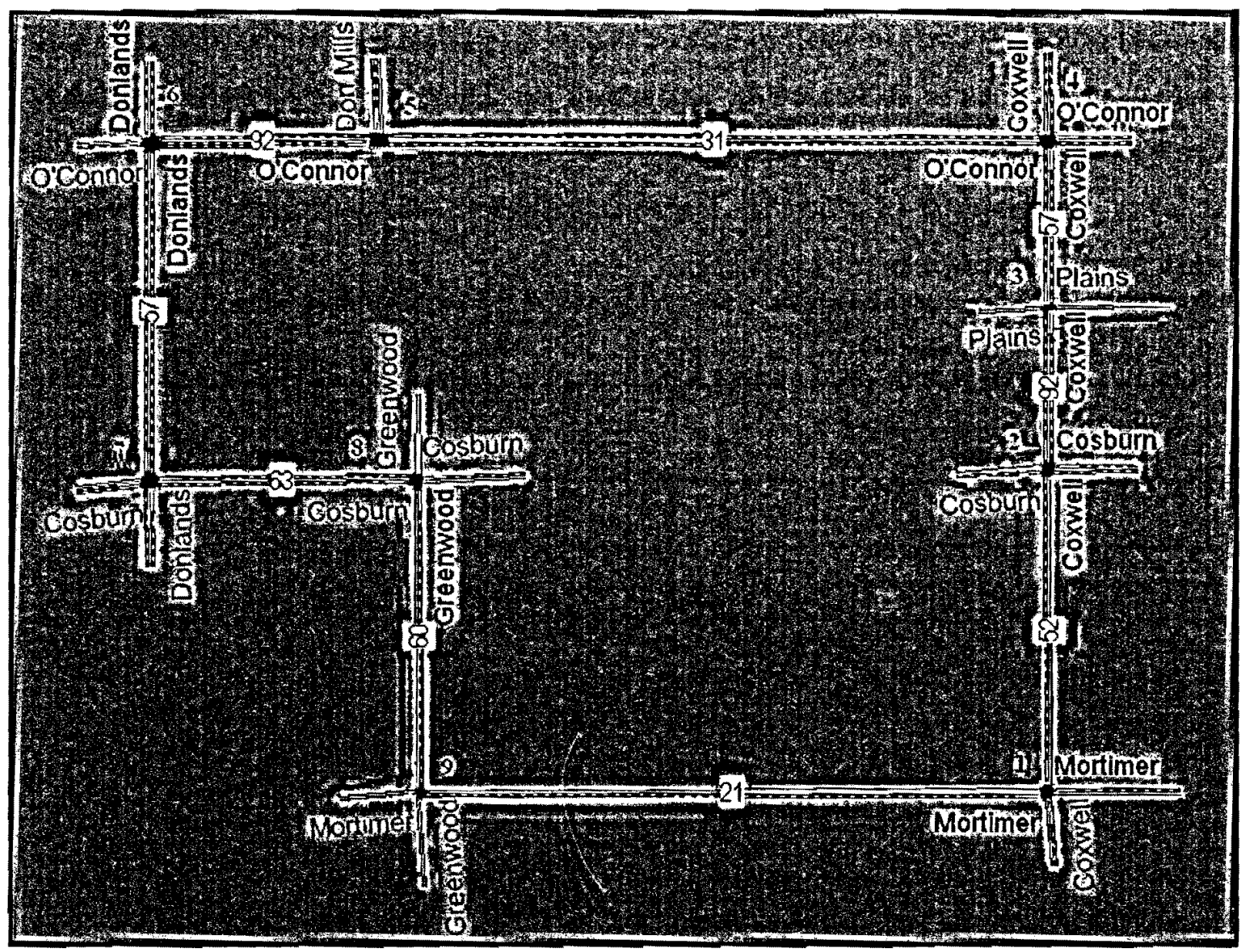

Figure 6: Synchro street network showing CF and street names

Synchro software calculates a factor to determine whether or not coordination between intersections should be done. This factor is called Coordinability Factor $(\mathrm{CF})$, which ranges from 0 to 100 or more. A CF value over 50 means that coordination is recommended. The higher the $\mathrm{CF}$, the more likely it is that segment will benefit from coordination.

CF takes travel times between intersections into account. Synchro recommends coordination if the travel time between intersections is less than 30 seconds ${ }^{(12)}$. Figure 6 shows CF for the streets on the intersection and it is clearly shown that streets joining intersections $1 \& 9$ and $4 \&$ 5 have CF equal to 21 and 31 respectively; thus Synchro does not recommend coordination for them. 


\subsection{Audit Report \& Observations}

This audit report shall include analysis of collision data for each of the intersections showing high number of collisions. Only after detailed study of conditions and factors that have contributed to the crashes, recommendations for treatment shall be given. I would start by giving a brief introduction of safety audit and enumerate the steps involved in the exercise.

A road safety audit is an in-depth engineering study of an existing road with the objective of identifying cost-effective countermeasures that shall improve traffic operations and road safety (15).

It generally has a standard operating procedure which is as follows:

- Network screening for identification of high risk corridor

- Data collection

- Detailed study of collision data for previous years

- Level of Service analysis

- Identification of safety concerns by site visits and comprehensive investigations

- Providing recommendations to better safety

Having reviewed the collision data (courtesy-City of Toronto Collision Reporting System) for the intersections in the chosen network, it is observed that Coxwell, O'Connor and Donlands are three streets that have high number of collisions. So before conducting a detailed safety audit for the same, I shall provide a graphical analysis of the collision data to understand the factors and conditions that have contributed to the frequency of collisions and accordingly appropriate countermeasures are enforced. 


\subsection{Collision Analysis}

1. Coxwell \& Mortimer:

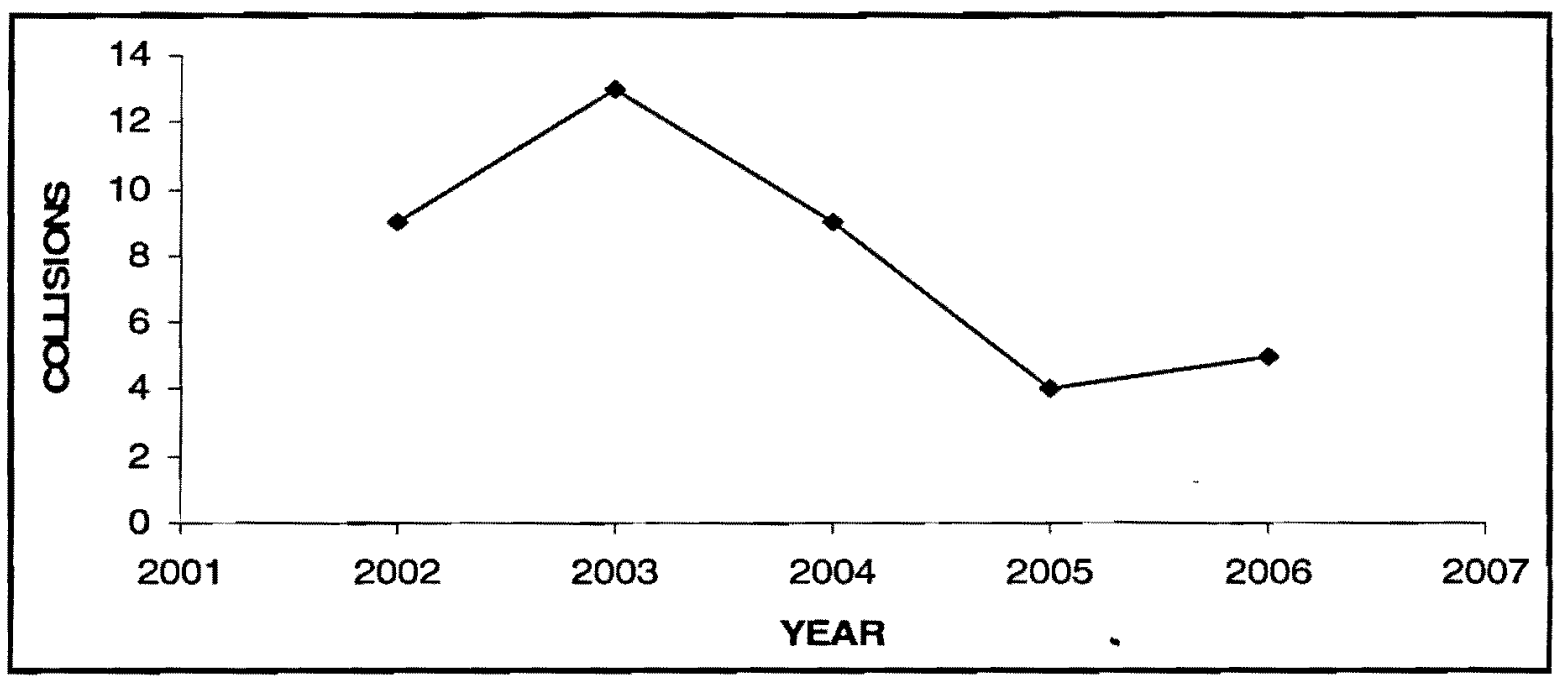

Figure 7: Collisions by year - Coxwell \& Mortimer

Figure 7 shows the historical trend for motor vehicle collisions at Coxwell \& Mortimer intersection. Data for years 2002-2006 has been provided by City of Toronto.

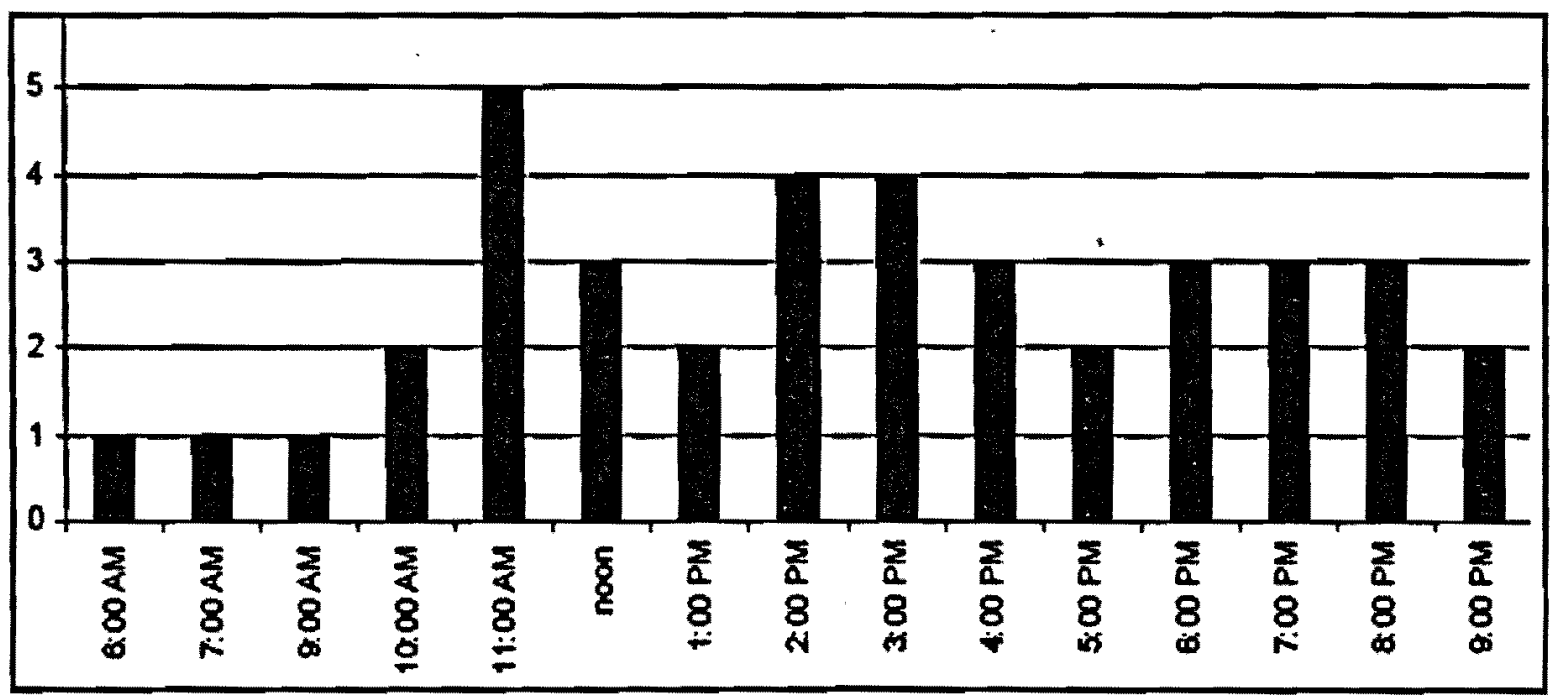

Figure 8: Collisions by hour- Coxwell \& Mortimer

Figure 8 shows times at which greater than normal collisions have taken place. 11 am and 2 to $3 \mathrm{pm}$ have been identified as times where as many as 13 collisions have taken place in last 5 years. 


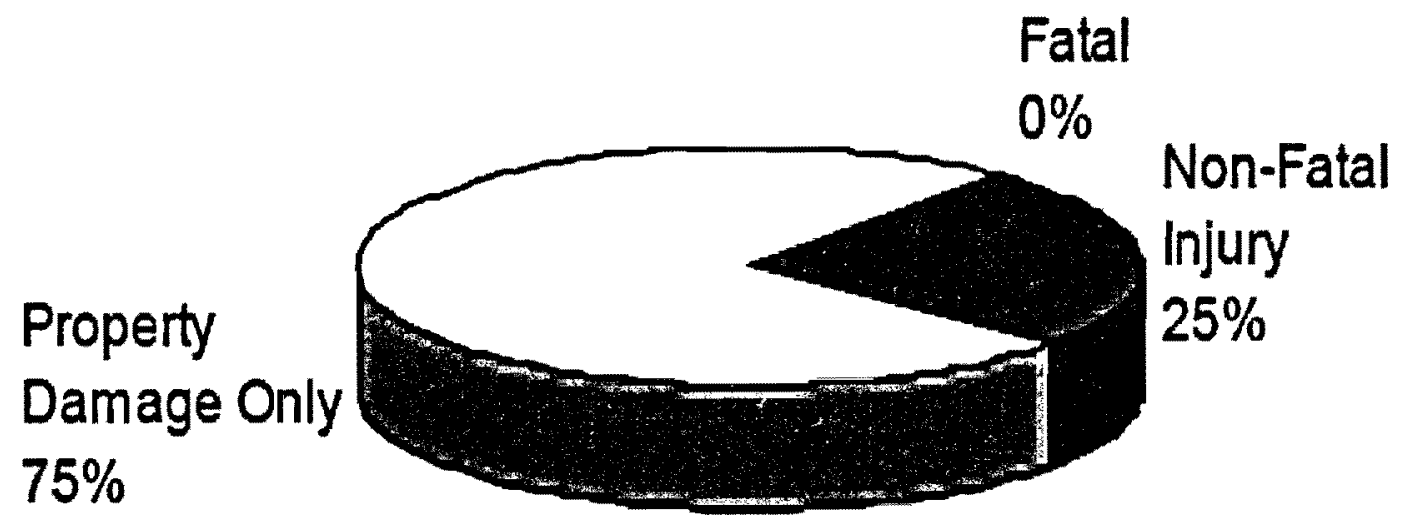

Figure 9: Collisions by classification - Coxwell \& Mortimer

This pie-diagram indicates that majority of crashes at Coxwell \& Mortimer have been property damage only and one-fourth have been non-fatal injury crashes. No fatalities have been recorded at this intersection.

Also observed is that majority of collisions have been rear end and turning movement and have taken place in dry conditions.

\begin{tabular}{|c|c|c|c|c|}
\hline \multicolumn{5}{|c|}{ Initial Impact by Class of Collision } \\
\hline \multirow[b]{2}{*}{ Initial Impact Type } & \multicolumn{3}{|c|}{ Class of Collision } & \multirow[b]{2}{*}{ Total } \\
\hline & Fatal & Personal Injury & Property Damage & \\
\hline Rear End & $\mathbf{0}$ & 3 & 13 & 16 \\
\hline Tuming Movement & 0 & 2 & 10 & 12 \\
\hline Angle & 0 & 2 & 3 & 5 \\
\hline Sideswipe & 0 & 0 & 4 & 4 \\
\hline Pedestrian Collision & 0 & 2 & 0 & 2 \\
\hline SMV Other & 0 & 1 & 0 & 1 \\
\hline Uncoded & 0 & 0 & 0 & 0 \\
\hline SMV Unattended Vehicle & 0 & 0 & 0 & 0 \\
\hline Other & 0 & 0 & 0 & 0 \\
\hline Cyclist Collision & 0 & 0 & 0 & 0 \\
\hline Approaching & 0 & 0 & 0 & 0 \\
\hline Total & 0 & 10 & 30 & 40 \\
\hline
\end{tabular}

Figure 10: Collisions by class of collision- Coxwell \& Mortimer 


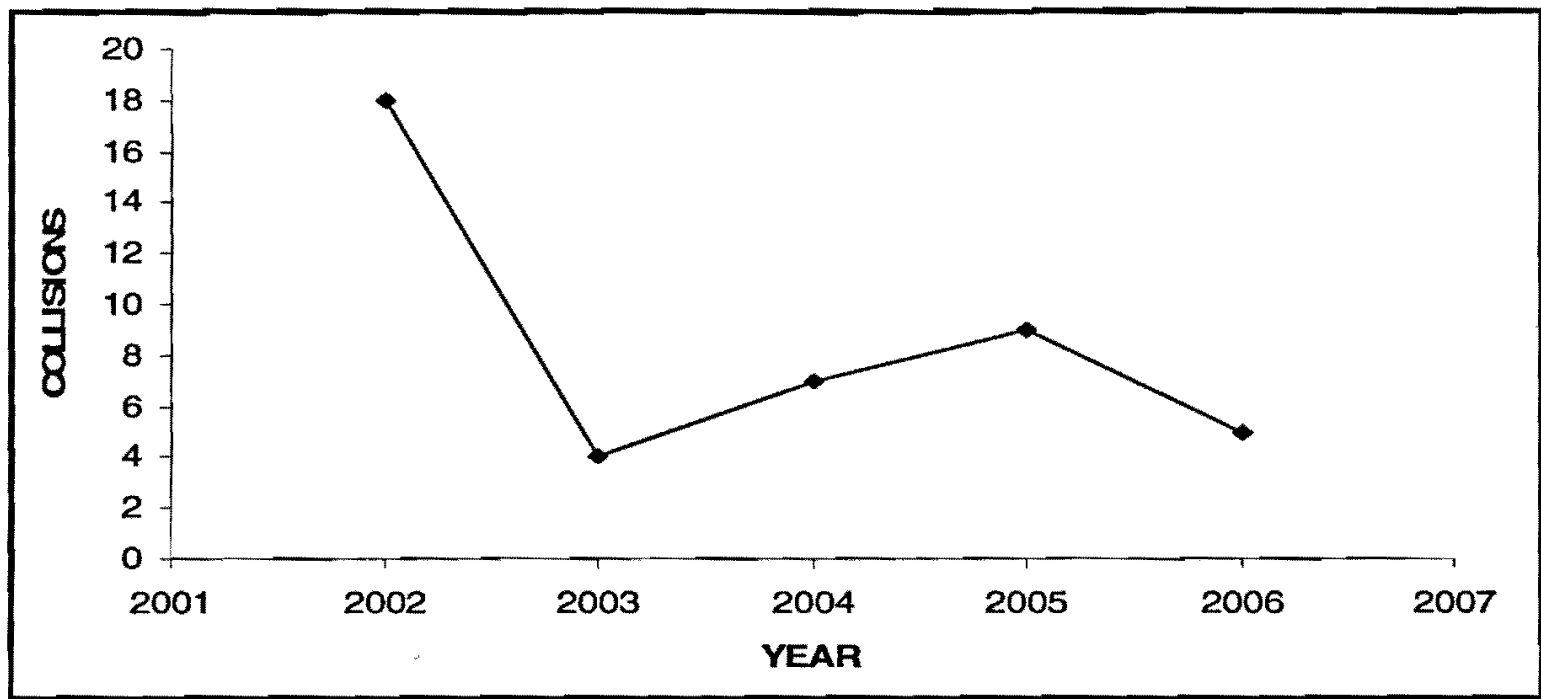

Figure 11: Collisions by year - Coxwell \& Cosburn

Figure 11 shows the historical trend for motor vehicle collisions at Coxwell \& Cosburn intersection from 2002 to 2006 inclusive.

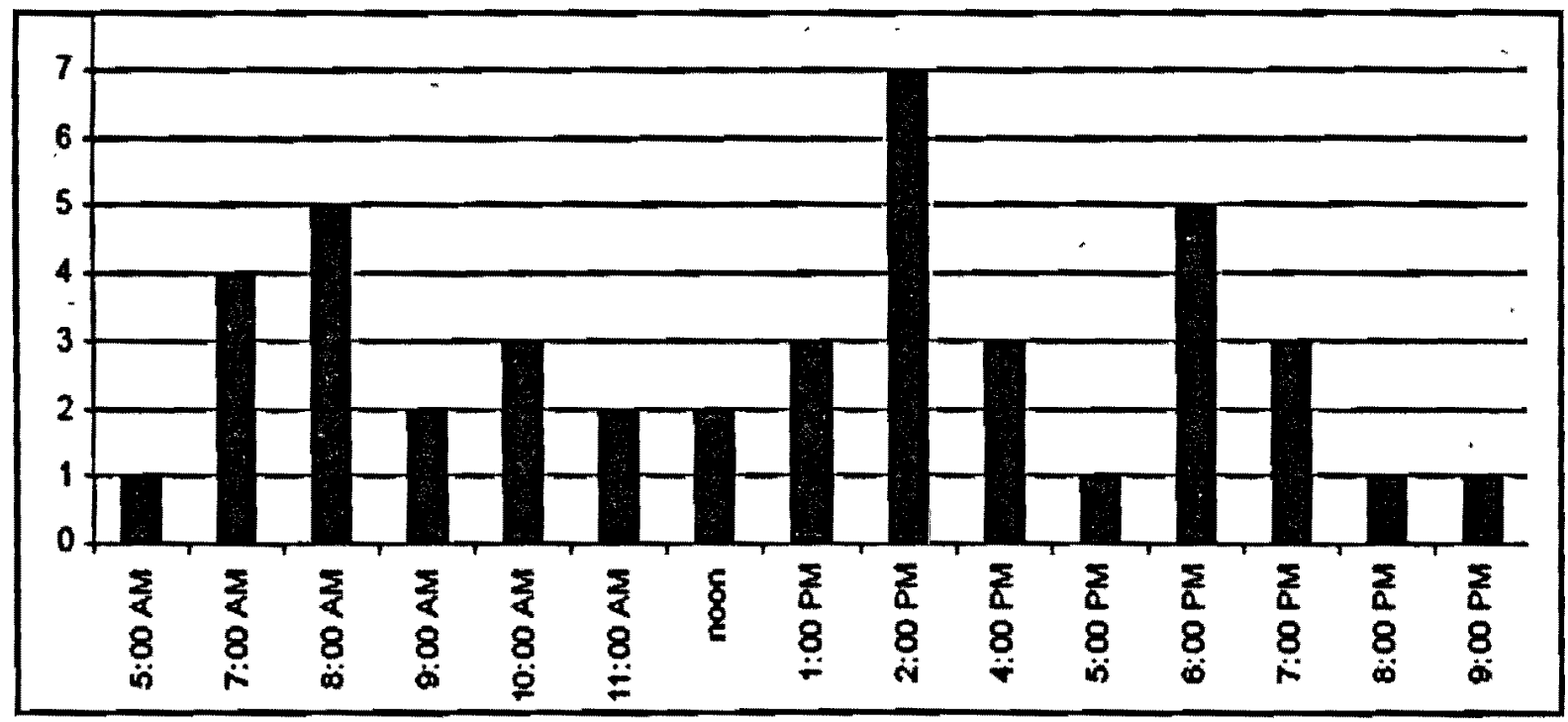

Figure 12: Collisions by hour - Coxwell \& Cosburn

Figure 12 shows that large portion of collisions have taken place in the morning and evening rush hour. A unique observation is that 7 collisions have taken place at $2 \mathrm{pm}$ in last 5 years.

This number could be attributed to the existence of East York Collegiate Institute right at the junction where student drop-off and pick up has been permitted at the road side. 


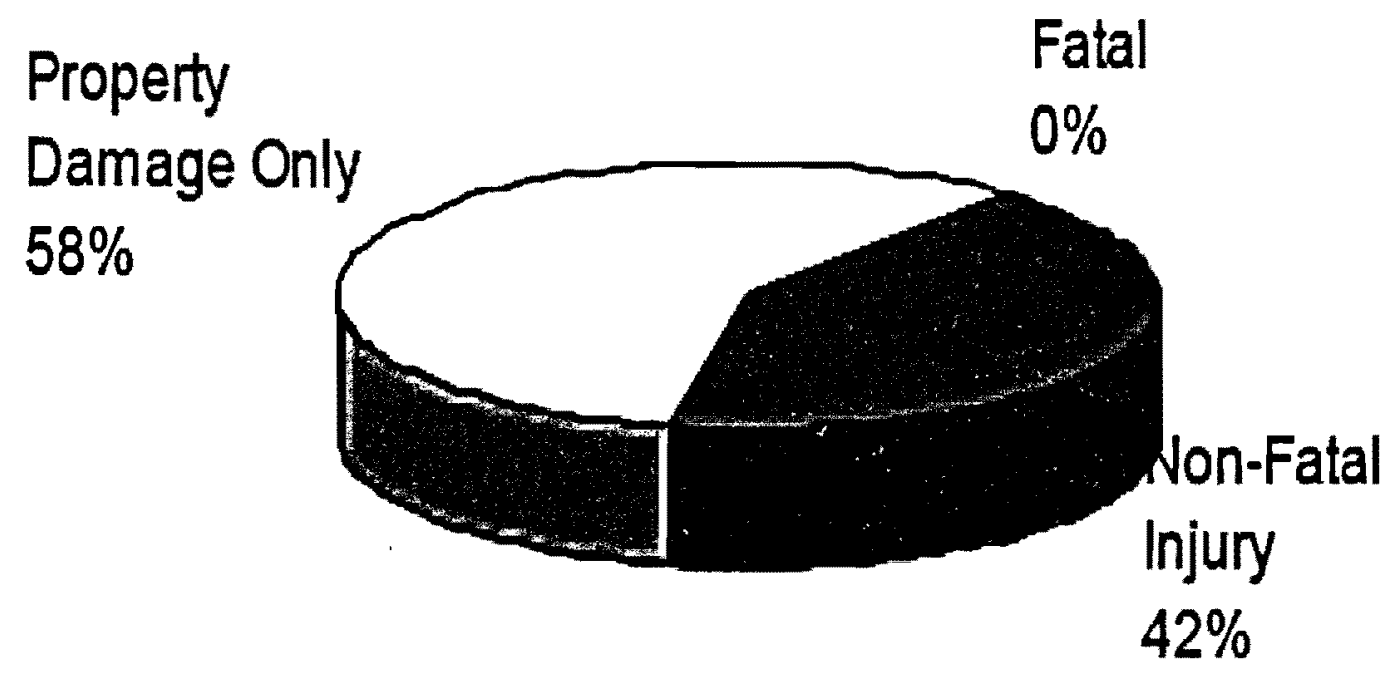

Figure 13: Collisions by classification- Coxwell \& Cosburn

Analysis shows that angle, rear end and turning movement accidents contribute heavily to the total number of accidents at this location. $58 \%$ and $42 \%$ collisions are property damage only and non-fatal injury respectively.

\begin{tabular}{|l|c|cc|c|}
\hline \multicolumn{3}{|l|}{ Initial Impact by Class of Collision } \\
\hline \multirow{2}{*}{ Initial Impact Type } & \multicolumn{3}{|c|}{ Class of Collision } & \multirow{2}{*}{ Total } \\
\cline { 2 - 5 } & Fatal & Personal Injury & Property Damage & 14 \\
Angle & 0 & 7 & 7 & 13 \\
Rear End & 0 & 5 & 8 & 10 \\
Turning Movement & 0 & 4 & 6 & 4 \\
Sideswipe & 0 & 0 & 4 & 1 \\
SMV Other & 0 & 1 & 0 & 1 \\
Cyclist Collision & 0 & 1 & 0 & 0 \\
Uncoded & 0 & 0 & 0 & 0 \\
SMV Unattended Vehicle & 0 & 0 & 0 & 0 \\
Pedestrian Collision & 0 & 0 & 0 & 0 \\
Other & 0 & 0 & 0 & 0 \\
Approaching & 0 & 0 & 0 & 43 \\
Total & 0 & 18 & 25 & \\
\hline
\end{tabular}

Figure 14: Collisions by class of collision- Coxwell \& Cosburn 
4. Coxwell \& O'Connor:

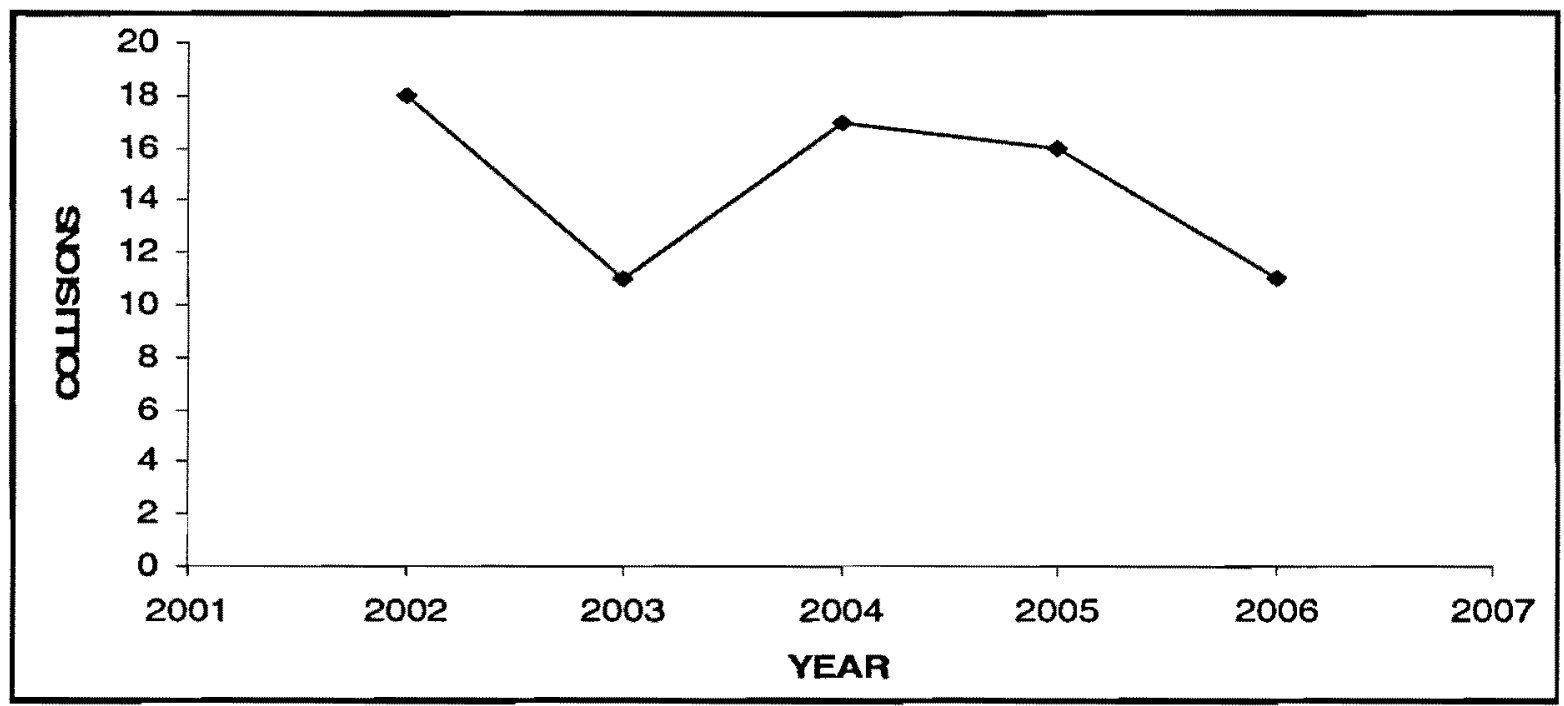

Figure 15: Collisions by year - Coxwell \& O'Connor

As many as 73 accidents have taken place at this intersection between years 2002 to 2006.52 out of those 73 have taken place in dry conditions.

Figure 16 shows that the morning rush hour has caused majority of crashes at the site.

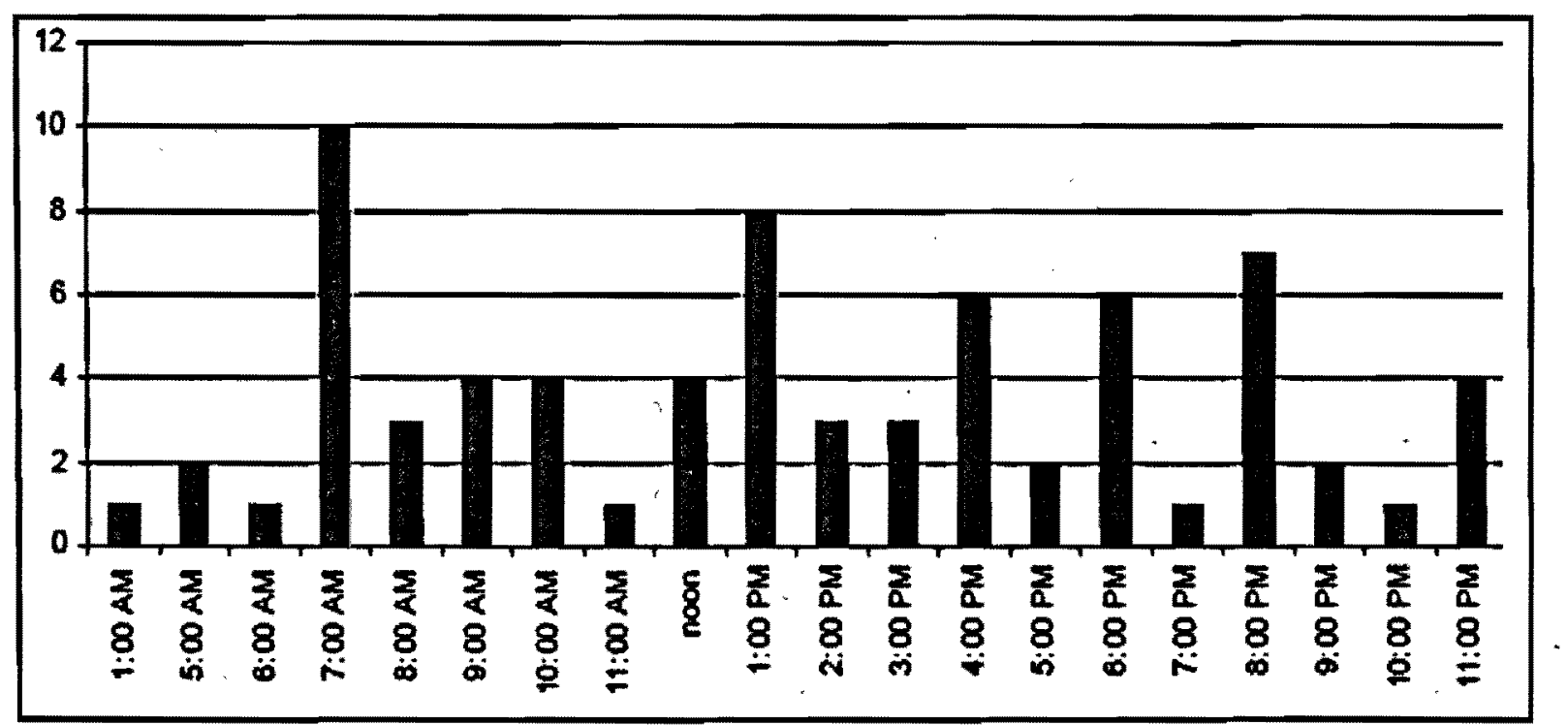

Figure 16: Collisions by hour - Coxwell \& O'Connor 


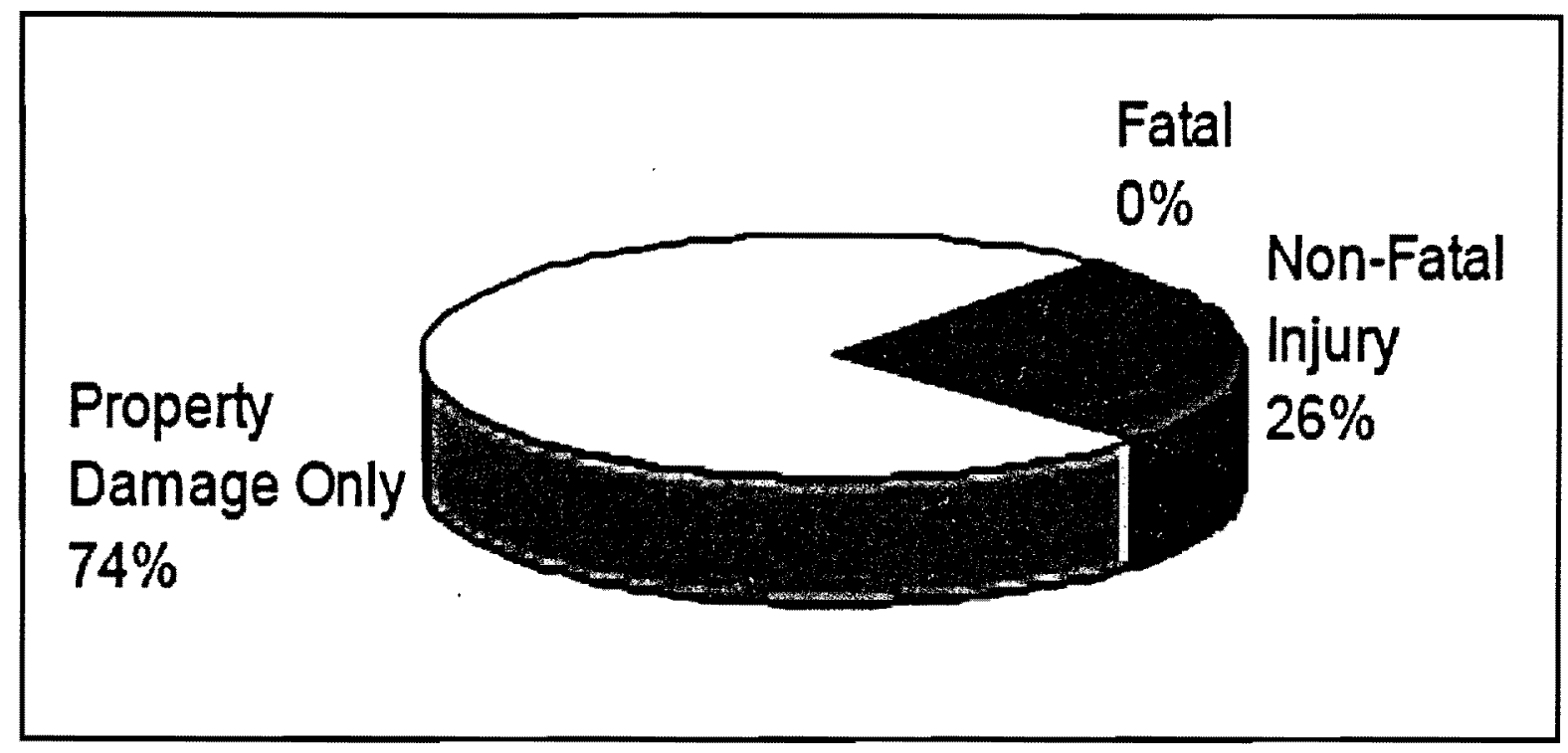

Figure 17: Collisions by classification - Coxwell \& O'Connor

There have been $74 \%$ and $26 \%$ PDO and non-fatal accidents respectively. Majority of these have been rear-end and turning movement accidents.

\begin{tabular}{|l|c|cc|c|}
\hline \multicolumn{3}{|l|}{ Initial Impact by Class of Collision } \\
\hline \multirow{2}{*}{ Initial Impact Type } & \multicolumn{3}{|c|}{ Class of Collision } & \multirow{2}{*}{ Total } \\
\cline { 2 - 5 } & Fatal & Personal Injury & Property Damage & 36 \\
\hline Rear End & 0 & 10 & 26 & 20 \\
Tuming Movement & 0 & 5 & 15 & 6 \\
Angle & 0 & 1 & 5 & 5 \\
Sideswipe & 0 & 0 & 5 & 2 \\
Pedestrian Collision & 0 & 2 & 0 & 2 \\
Approaching & 0 & 0 & 2 & 1 \\
SMV Other & 0 & 0 & 1 & 1 \\
Cyclist Collision & 0 & 1 & 0 & 0 \\
Uncoded & 0 & 0 & 0 & 0 \\
SMV Unattended Vehicle & 0 & 0 & 0 & 0 \\
Other & 0 & 0 & 0 & 73 \\
Total & 0 & 19 & 54 & \\
\hline
\end{tabular}

Figure 18: Collisions by class of collision - Coxwell \& O'Connor 


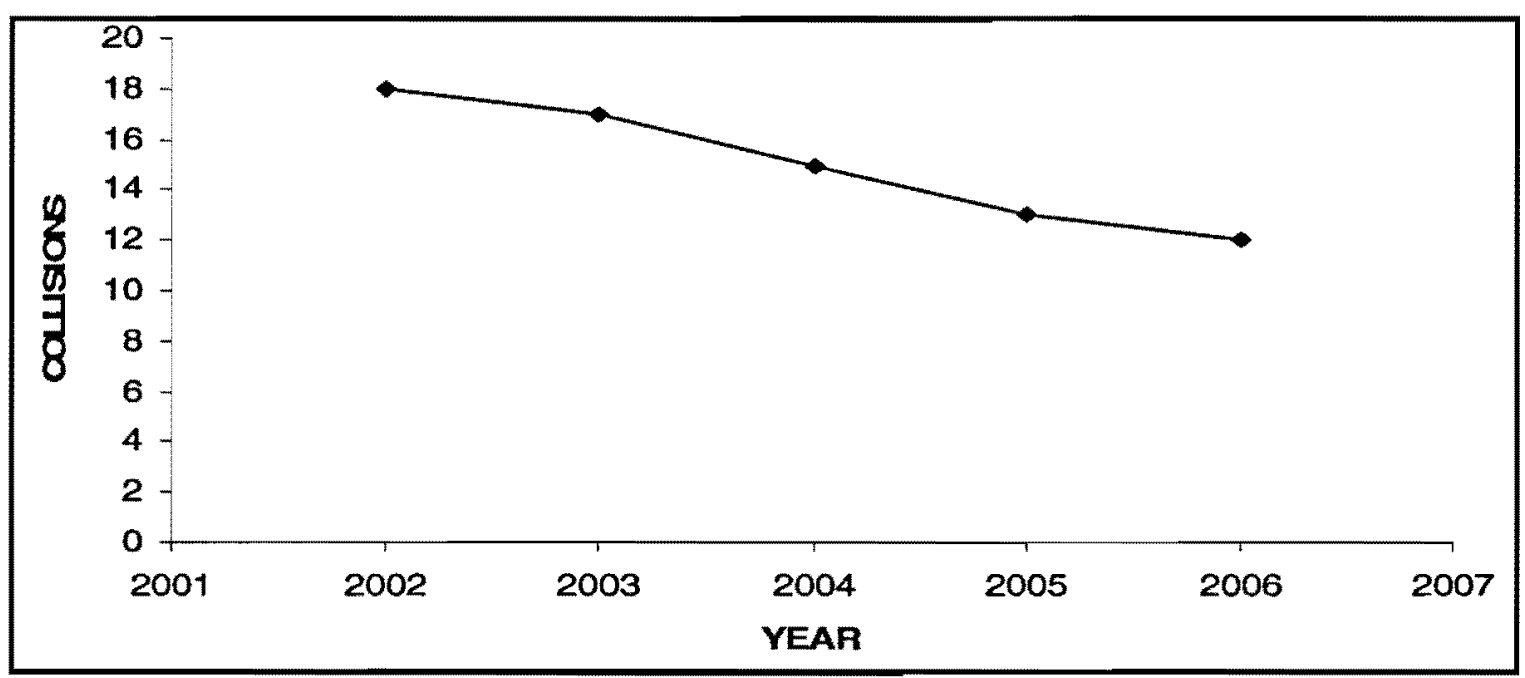

Figure 19: Collisions by year - O'Connor \& Don Mills

Figure 19 shows the historical trend for motor vehicle collisions at O'Connor \& Don Mills intersection from 2002 to 2006 inclusive. The graph shows a study decline in the number of collisions. A total of 75 accidents have taken place at this intersection.

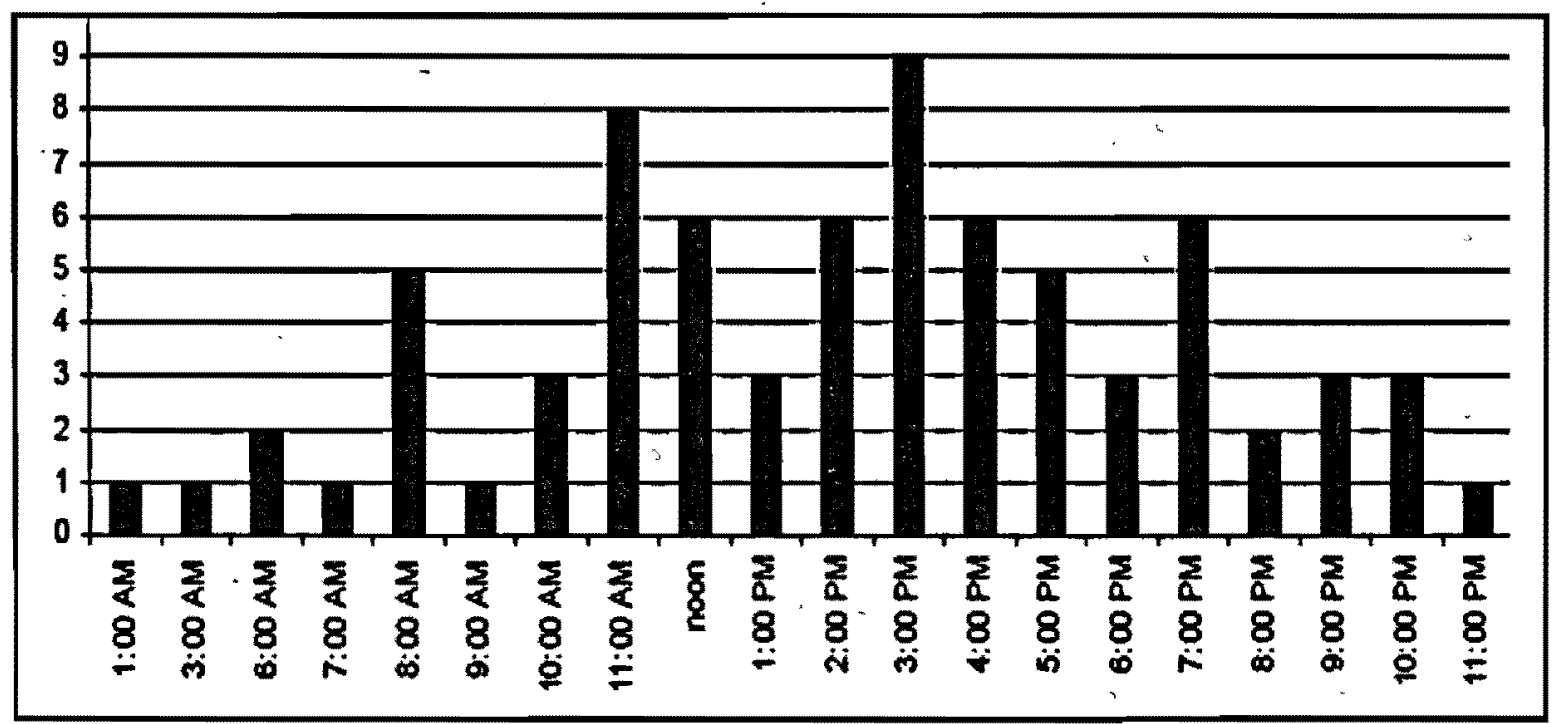

Figure 20: Collisions by hour - O'Connor \& Don Mills

Bar graph shows that the accidents are fairly distributed. The number reduces only between $8 \mathrm{pm}$ to morning $7 \mathrm{pm}$. 


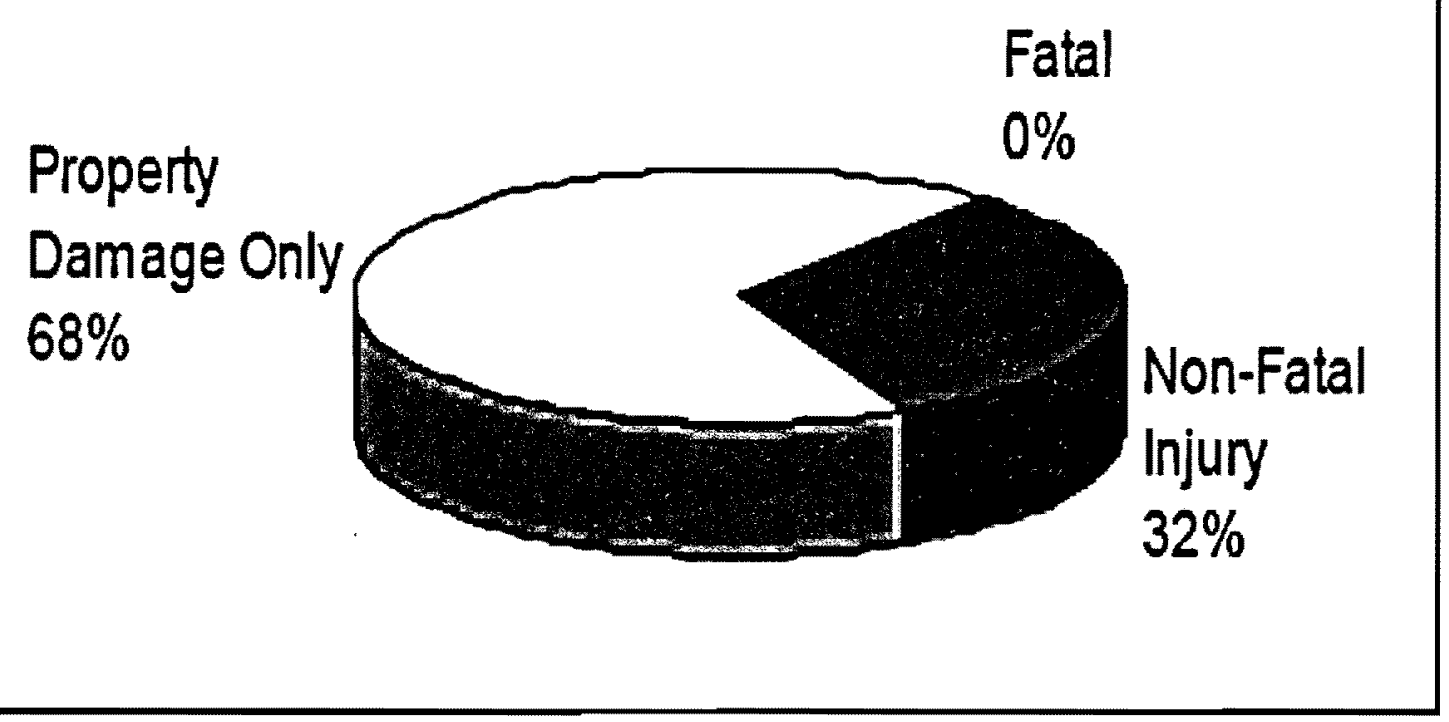

Figure 21: Collisions by classification -O'Connor \& Don Mills

PDO contribute to $68 \%$ of all the accidents and remaining $32 \%$ are non-fatal injury accidents. This intersection also has a high proportion of rear-end and turning movement collisions.

\begin{tabular}{|l|c|cc|c|}
\hline \multicolumn{3}{|l|}{ Initial Impact by Class of Collision } \\
\hline \multirow{2}{*}{ Initial Impact Type } & \multicolumn{3}{c|}{ Class of Collision } & \multirow{2}{*}{ Total } \\
\cline { 2 - 5 } & Fatal & Personal Injury & Property Damage & Tor \\
\hline Rear End & 0 & 18 & 26 & 44 \\
Turning Movement & 0 & 4 & 14 & 18 \\
Angle & 0 & 1 & 7 & 8 \\
Sideswipe & 0 & 0 & 4 & 4 \\
Approaching & 0 & 1 & 0 & 1 \\
Uncoded & 0 & 0 & 0 & 0 \\
SMV Unattended Vehicle & 0 & 0 & 0 & 0 \\
SMV Other & 0 & 0 & 0 & 0 \\
Pedestrian Collision & 0 & 0 & 0 & 0 \\
Other & 0 & 0 & 0 & 0 \\
Cyclist Collision & 0 & 0 & 0 & 0 \\
Total & 0 & 24 & 51 & 75 \\
\hline
\end{tabular}

Figure 22: Collisions by class of collision - O'Connor \& Don Mills 


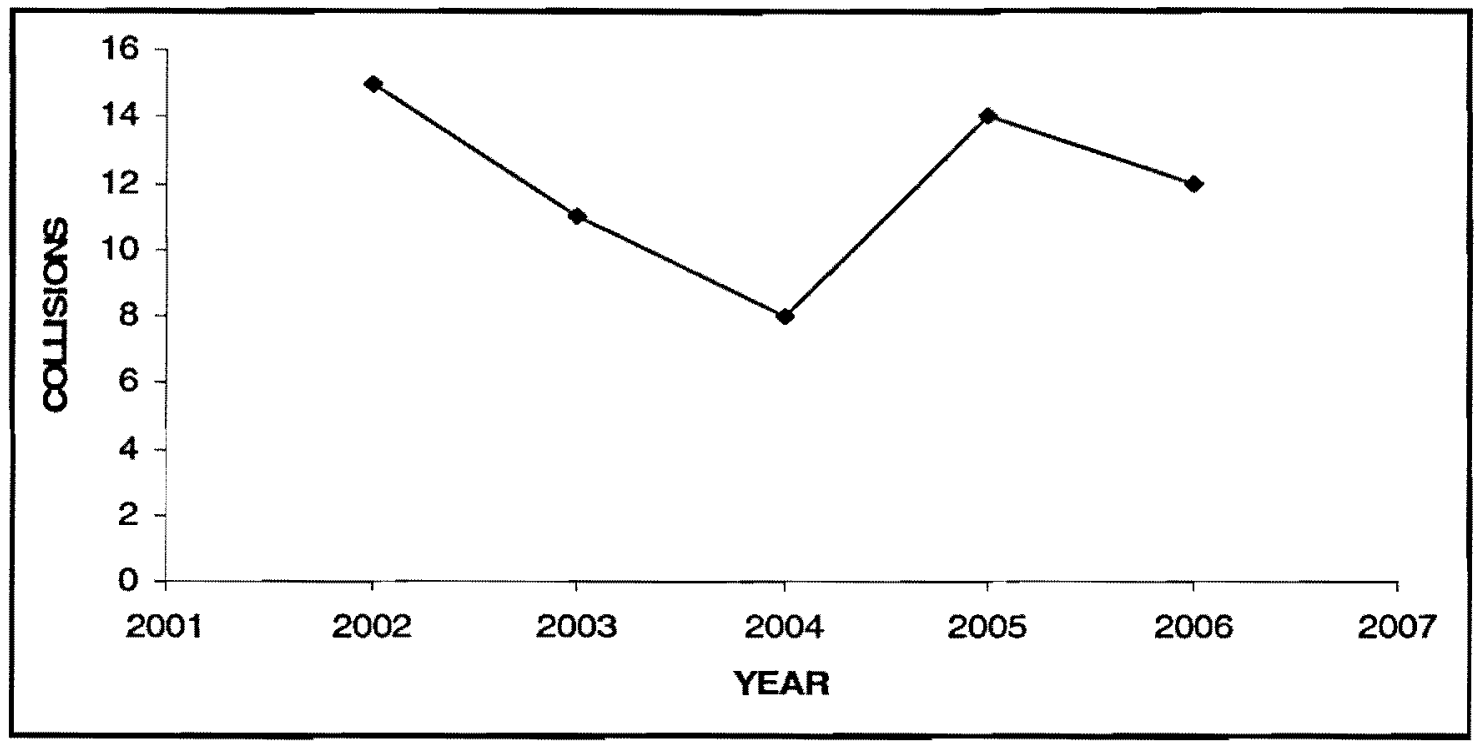

Figure 23: Collisions by year - O' Connor \& Donlands

A total of 60 crashes have taken place in years 200-2006 at O' Connor \& Donlands. 49 of those 60 have been in dry conditions. The trend of crashes is as depicted in the graph.

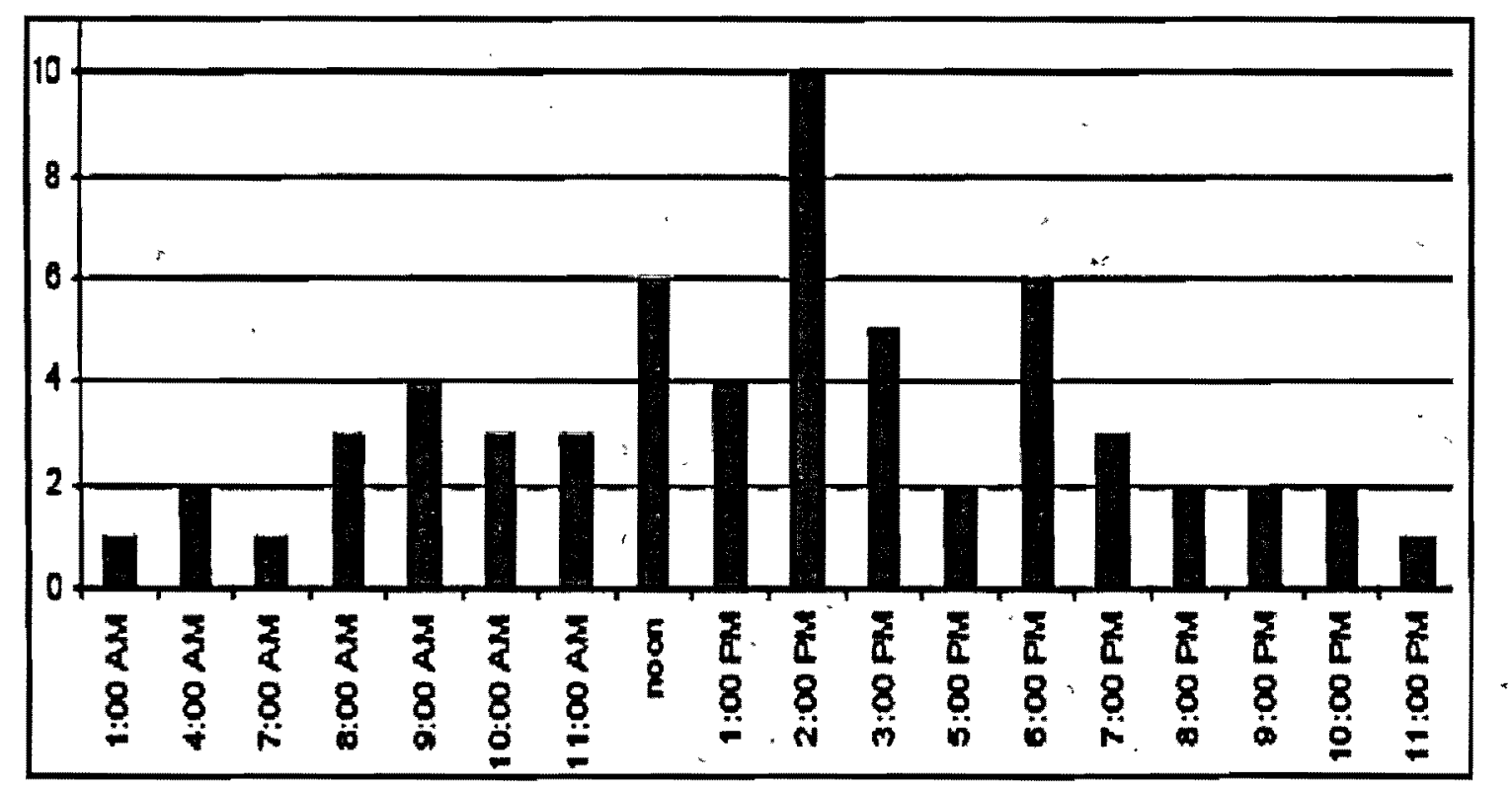

Figure 24: Collisions by hour - O' Connor \& Donlands

The bar graph in Figure 24 shows that extremely high number of crashes have occurred in the afternoon. 


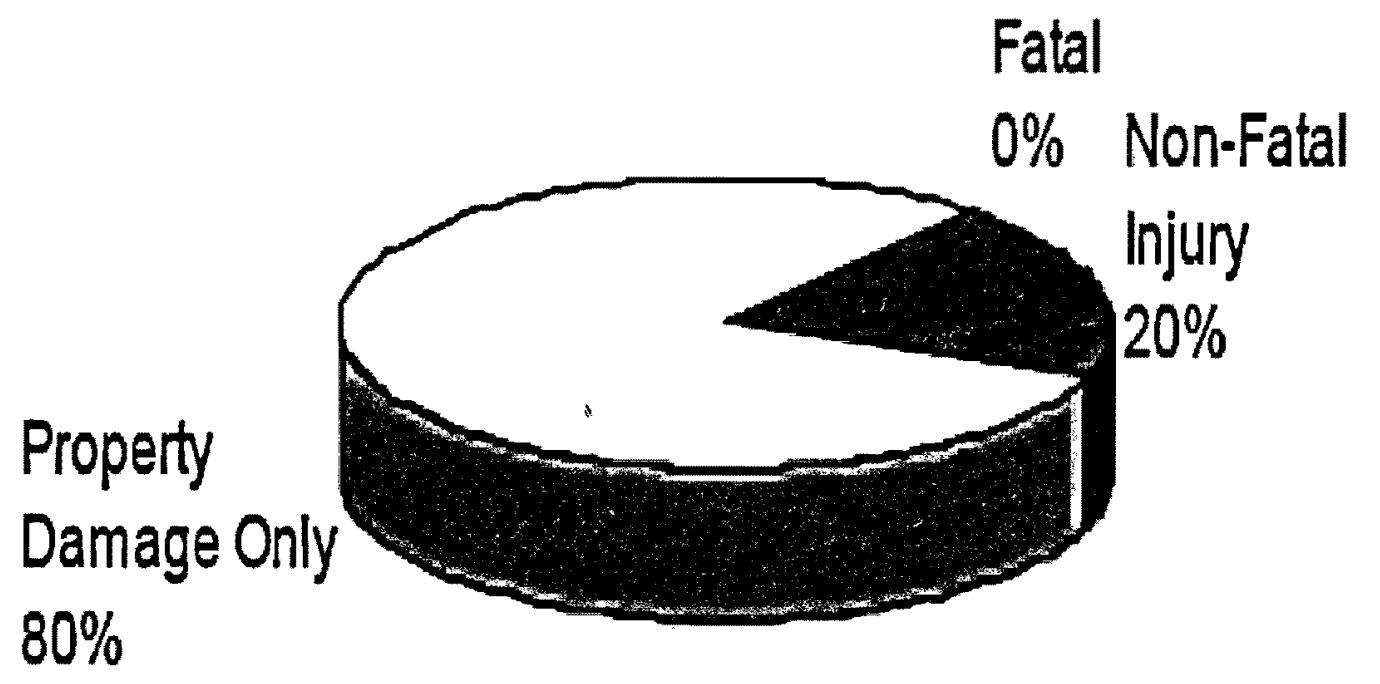

Figure 25: Collisions by classification - O' Connor \& Donlands

There have been $80 \%$ and $20 \%$ PDO and non-fatal accidents at $O^{\prime}$ Connor \& Donlands respectively. Majority of these have been rear-end and turning movement accidents.

\begin{tabular}{|c|c|c|c|c|}
\hline nitial Impact by Class of & ion & & & \\
\hline \multirow[b]{2}{*}{ Initial Impact Type } & \multicolumn{3}{|c|}{ Class of Collision } & \multirow[b]{2}{*}{ Total } \\
\hline & Fatal & Personal Injury & Property Damage & \\
\hline Rear End & $\overline{0}$ & $\overline{3}$ & $\overline{22}$ & 25 \\
\hline Tuming Movement & 0 & 4 & 16 & 20 \\
\hline Sideswipe & 0 & 0 & 6 & 6 \\
\hline Pedestrian Collision & 0 & 4 & 0 & 4 \\
\hline Angle & 0 & 1 & 2 & 3 \\
\hline SMV Other & 0 & 0 & 1 & 1 \\
\hline Approaching & 0 & 0 & 1 & 1 \\
\hline Uncoded & 0 & 0 & 0 & 0 \\
\hline SMV Unattended Vehicle & 0 & 0 & 0 & $\mathbf{0}$ \\
\hline Other & 0 & 0 & 0 & 0 \\
\hline Cyclist Collision & 0 & 0 & 0 & $\mathbf{0}$ \\
\hline Total & $\mathbf{0}$ & 12 & 48 & 60 \\
\hline
\end{tabular}

Figure 26: Collisions by class of collision - O' Connor \& Donlands 


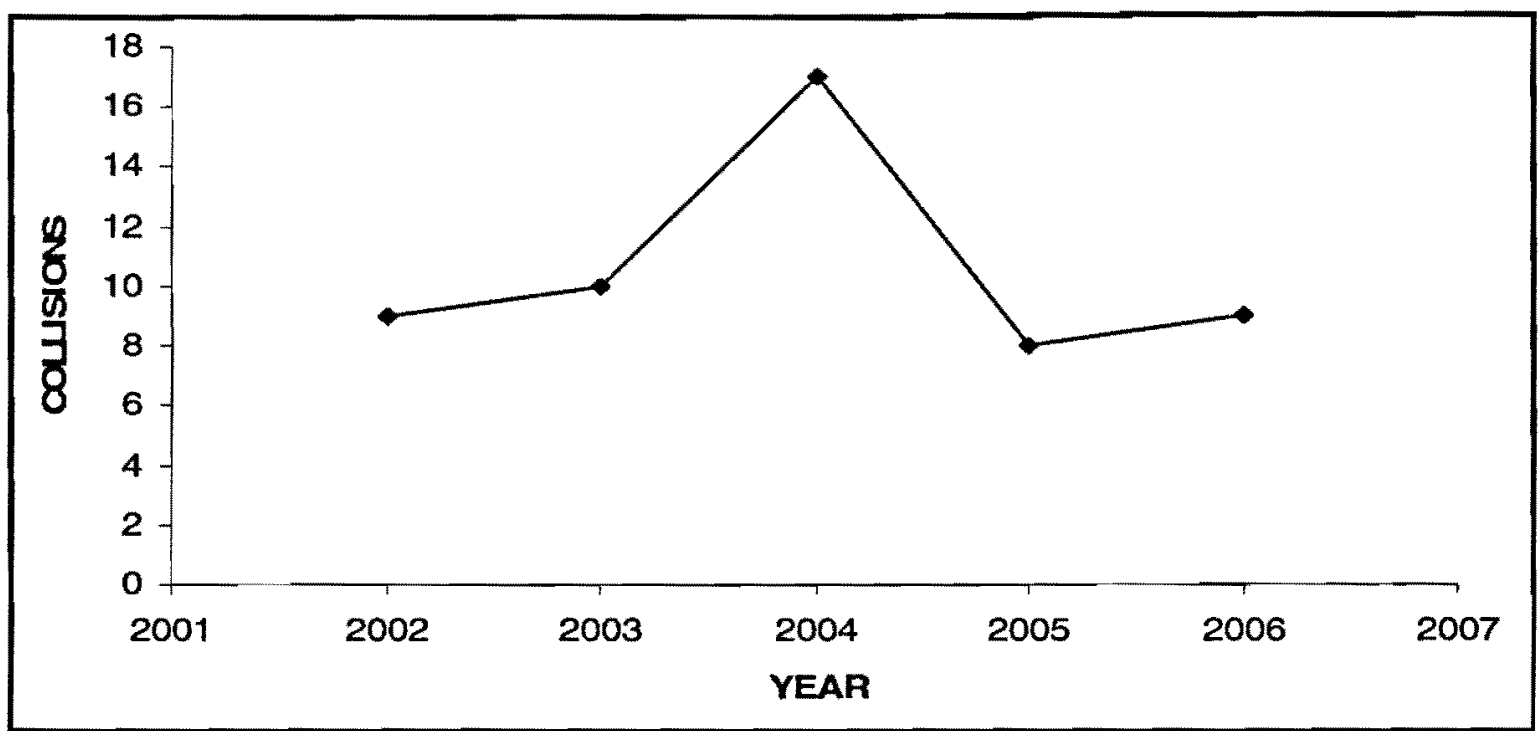

Figure 27: Collisions by hour- Cosburn \& Donlands

As many as 53 accidents have taken place at this intersection between years 2002 to 2006.13 and 15 out of the total 53 have taken place in dry and wet conditions respectively.

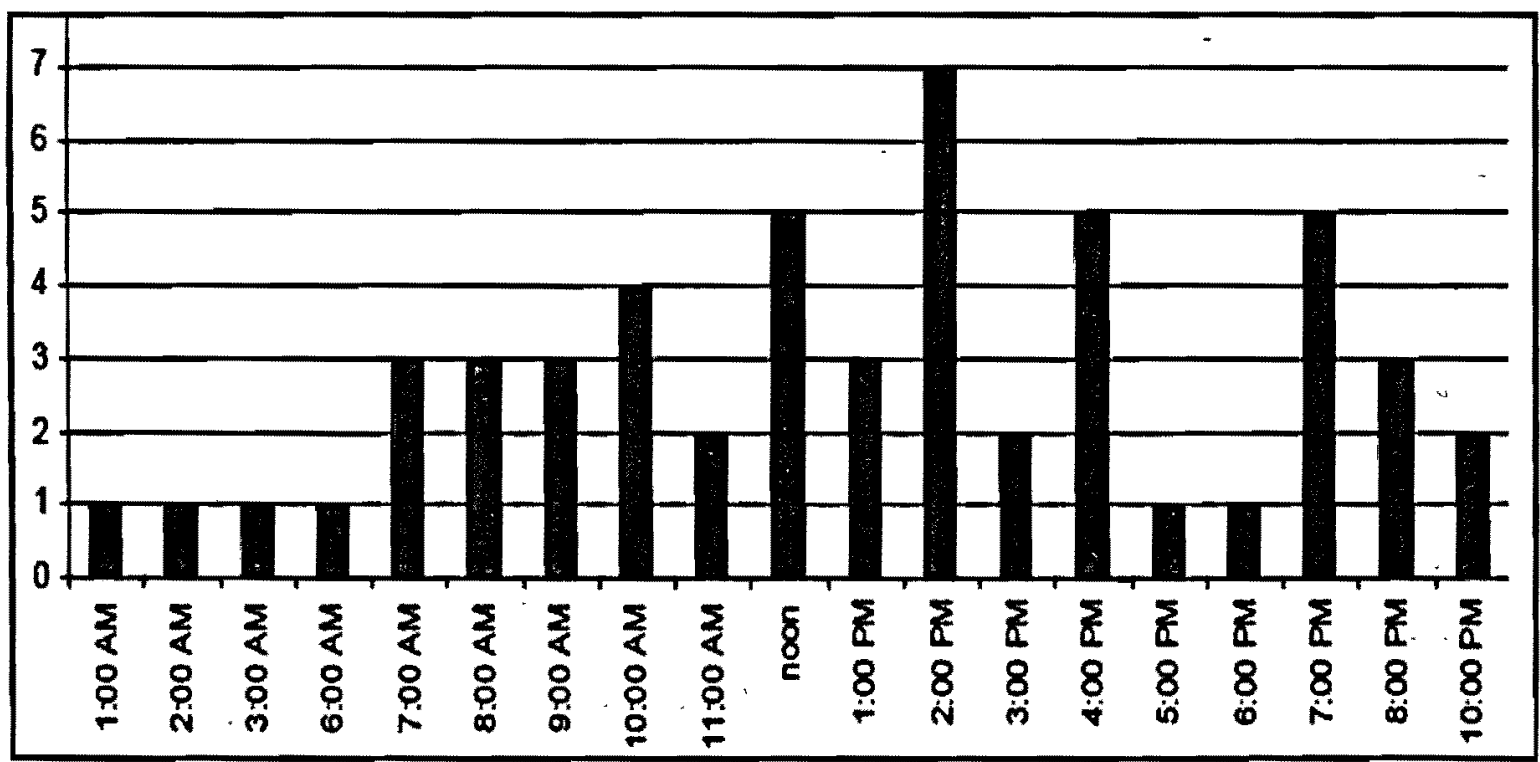

Figure 28: Collisions by hour - Cosburn \& Donlands

Figure 28 shows a fairly distributed accident pattern for the whole day, the number of accidents going up as the day progresses with a maximum of 7 accidents occurring at $2 \mathrm{pm}$. 


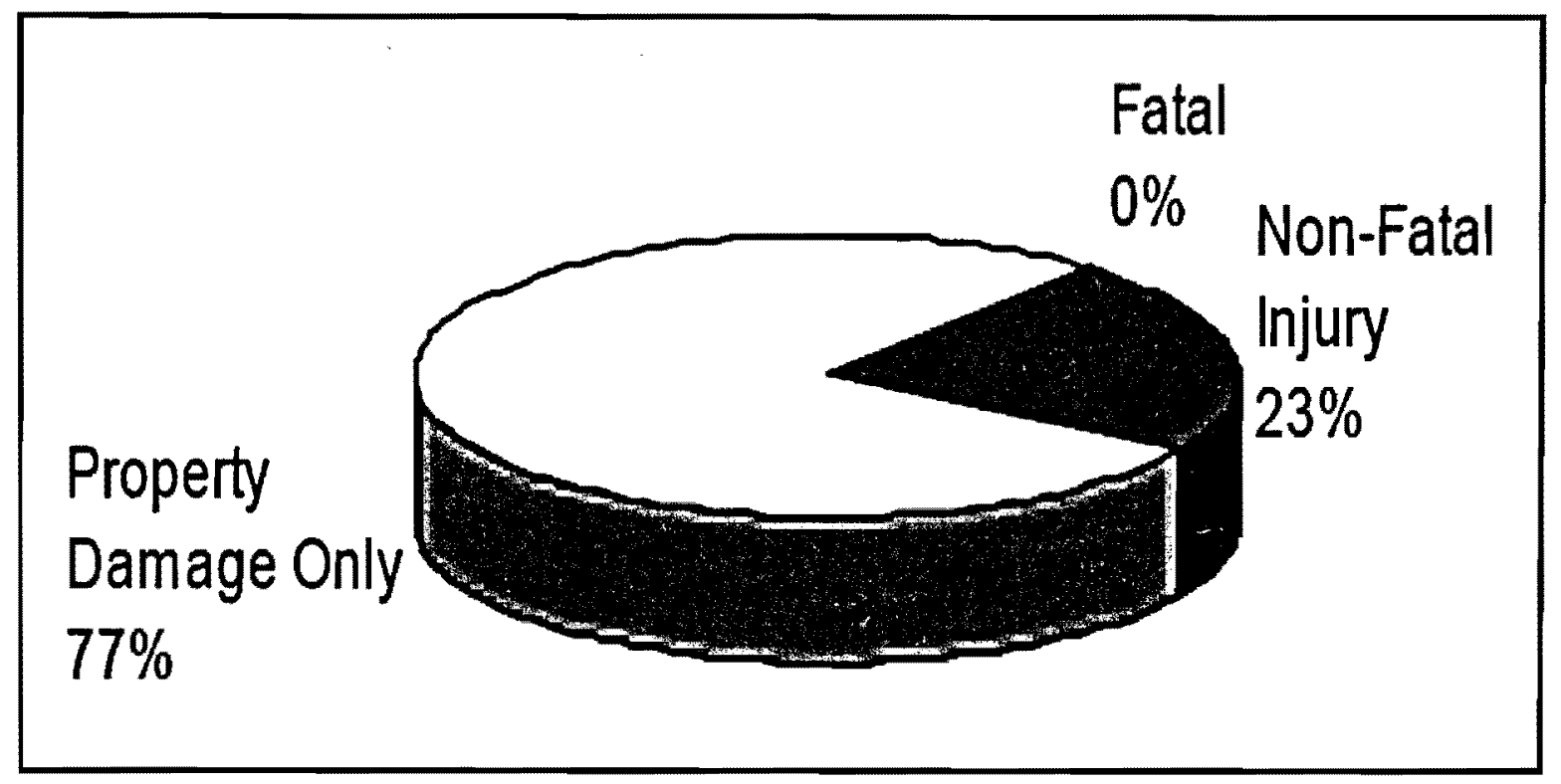

Figure 29: Collisions by classification - Cosburn \& Donlands

PDO contribute to $77 \%$ of all the accidents and remaining $23 \%$ are non-fatal injury accidents.

This intersection has a high proportion of rear-end turning movement and angle collisions.

\begin{tabular}{|c|c|c|c|c|}
\hline \multicolumn{5}{|c|}{ Initial Impact by Class of Collision } \\
\hline \multirow[b]{2}{*}{ Initial Impact Type } & \multicolumn{3}{|c|}{ Class of Collision } & \multirow[b]{2}{*}{ Total } \\
\hline & Fatal & Personal Injury & Property Damage & \\
\hline Tuming Movement & 0 & 4 & 13 & 17 \\
\hline Rear End & 0 & 5 & 12 & 17 \\
\hline Angle & 0 & 3 & 10 & 13 \\
\hline Sideswipe & 0 & 0 & 3 & 3 \\
\hline SMV Other & 0 & 0 & 2 & 2 \\
\hline Pedestrian Collision & 0 & $\mathbf{0}$ & 1 & 1 \\
\hline Uncoded & 0 & 0 & 0 & $\mathbf{0}$ \\
\hline SMV Unattended Vehicle & 0 & 0 & 0 & $\mathbf{0}$ \\
\hline Other & 0 & 0 & 0 & 0 \\
\hline Cyclist Collision & 0 & 0 & 0 & 0 \\
\hline Approaching & 0 & 0 & 0 & $\mathbf{0}$ \\
\hline Total & $\mathbf{0}$ & 12 & 41 & 53 \\
\hline
\end{tabular}

Figure 30: Collisions by class of collision - Cosburn \& Donlands 


\subsection{Site visit:}

The first site visit took place on March 2, 2007 in the evening peak hour. The purpose of the visit was to collect physical data, lane configuration, speed limits and to conduct a general reconnaissance of the region.

The second visit was made on April 6, 2007 from 12 noon to 4 PM. Detailed investigation of road conditions was done and potential safety hazards were recorded. Pictures of the site were taken so that careful analysis could be done and fitting corrections could be put into effect.

The safety audit site is in a mixed residential and a commercial area with ever increasing traffic growth because of proximity to Don Valley Parkway and the existence of a hospital and school in the area. Poor level of service and starvation delays at most of the intersections especially on the $\mathrm{O}^{\prime}$ Connor avenue is a cause for concern.

\subsection{General Findings of the Audit:}

The site investigation found that the following were the most common safety concerns in the area audited:

- Inadequate pavement markings

- Insufficient lighting

- Improper land development (property lines extended right up to the pavement edge obstructing turning vehicle's sight at the junctions)

- Too many driveways close to intersection

- Poor location of signs

These along with poorly performing signals were the deficiencies found in the survey; delays and congestion contributed to erratic driver behavior and increased safety risks as it was observed that large number of collisions occurred in the morning rush hour.

I shall now provide intersection specific findings and recommendations based on software analysis and site investigation. The hazards have been shown clearly in the pictures that follow. 


\section{Coxwell \& Mortimer:}

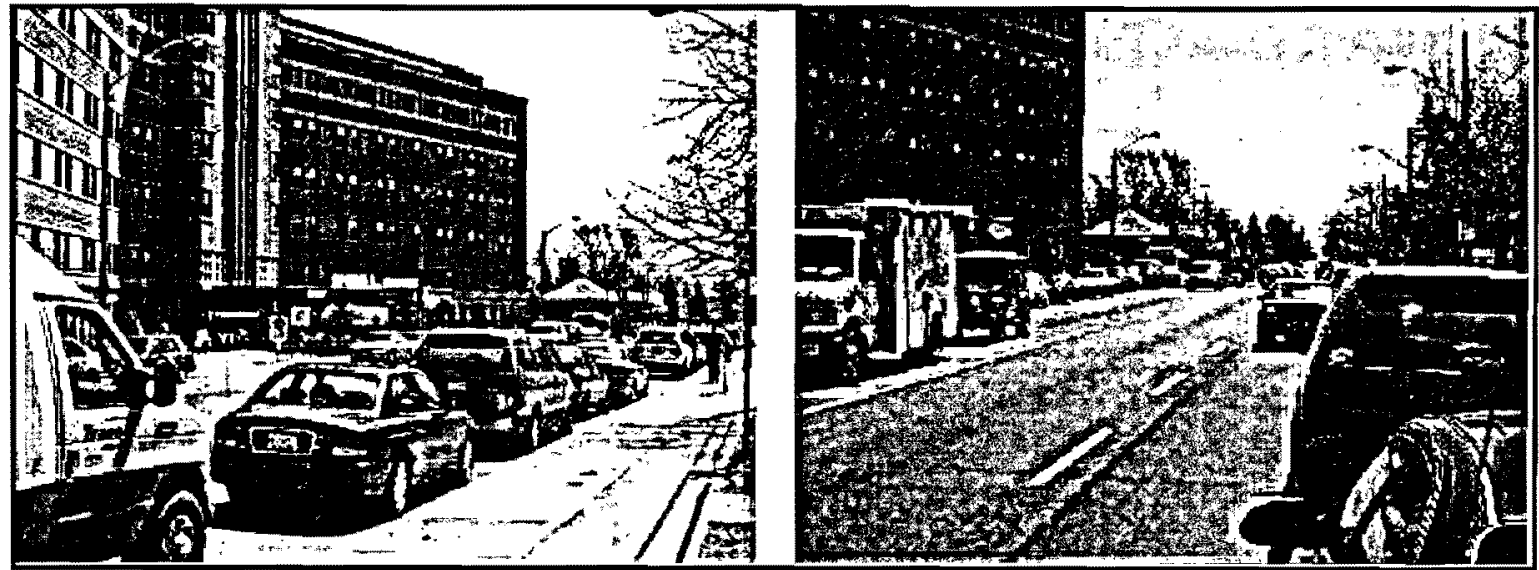

Figures 31 \& 32: Road side parking at Coxwell \& Mortimer

Figures 31 and 32 show that there is parking allowed on both sides of the road which limits the capacity and because there is no center lane provided for vehicle entry to the hospital, turning vehicles block the road for through traffic.
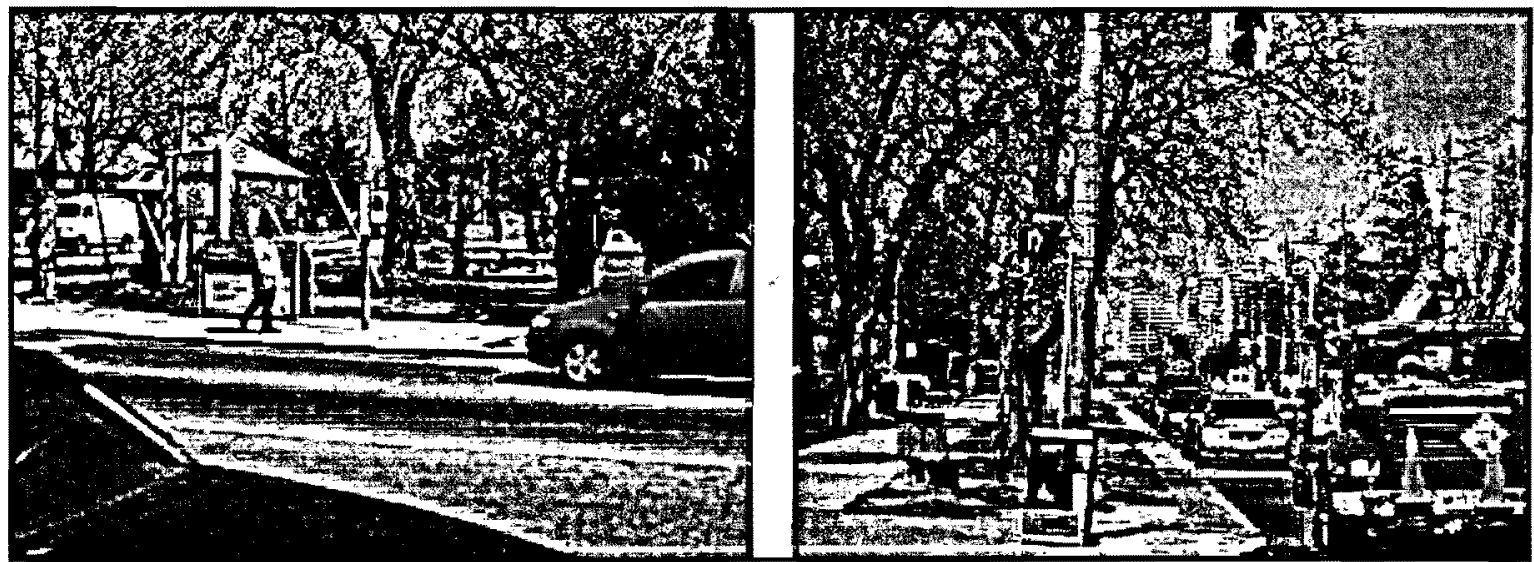

Figures 33 \& 34: No provision for bus bay - Coxwell \& Mortimer

Figure 33 shows bus stop right at the intersection and no bus bay provided whereas there is plenty of available space, also made clear by Figure 34 . Recommendations for this intersection are provision of bus bays to allow free movement of through traffic. This shall increase throughput and reduce delays. Also provision of center lane for entry and exit from the hospital shall better safety as conflicts shall be reduced. 


\section{Coxwell \& Cosburn:}

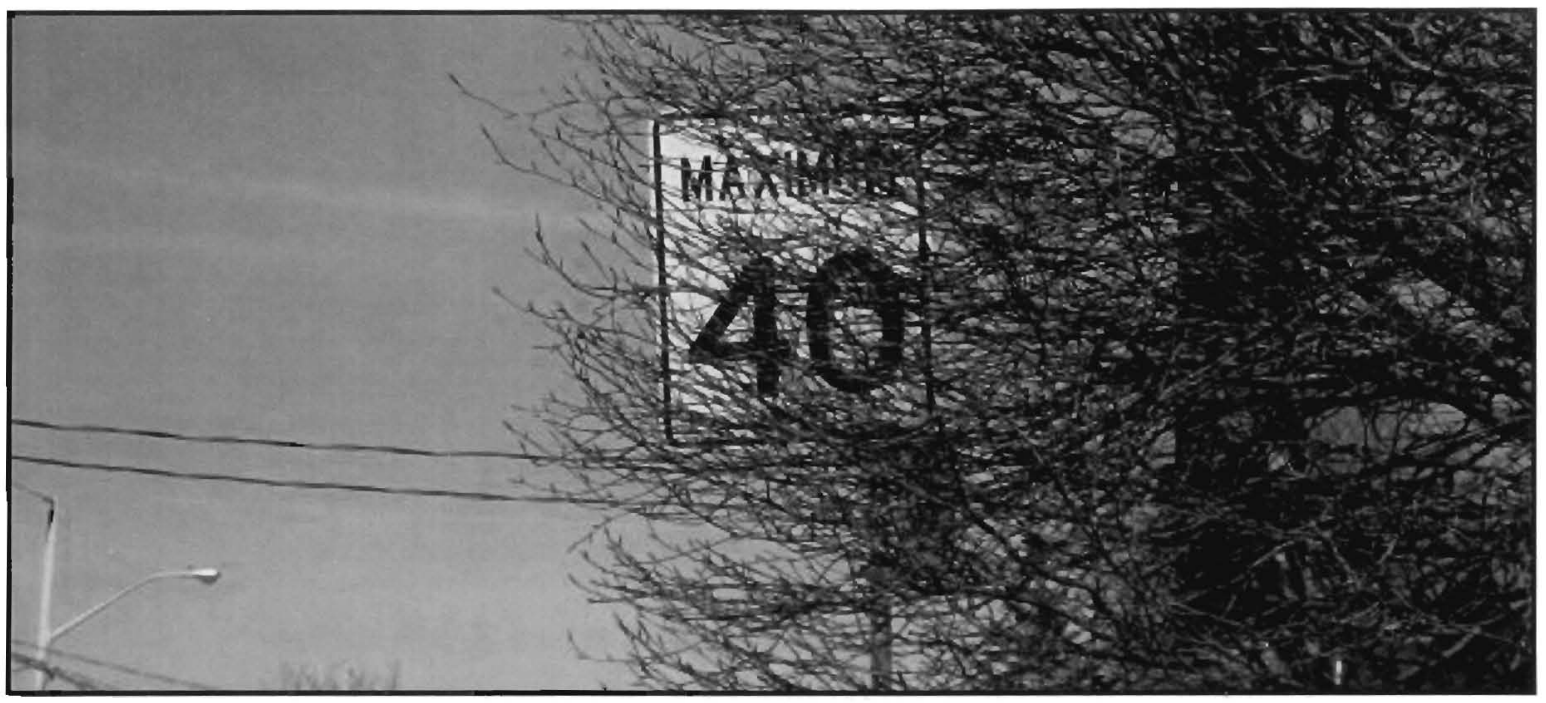

Figure 35: Sign hidden by overgrown tree - Coxwell \& Cosburn

A number of recommendations can be suggested to reduce collisions at this intersection Signs need to be made more conspicuous; Figure 35 shows that speed limit sign has been blocked by an overgrown tree. A big flaw observed at the intersection was student pick up and drop-off area at the major street which blocks traffic and causes unnecessary delays (Refer Figure 36). The East York Collegiate Institute has a big parking lot which remains underutilized. Road side stopping poses a potential safety hazard which can be done away with easily.

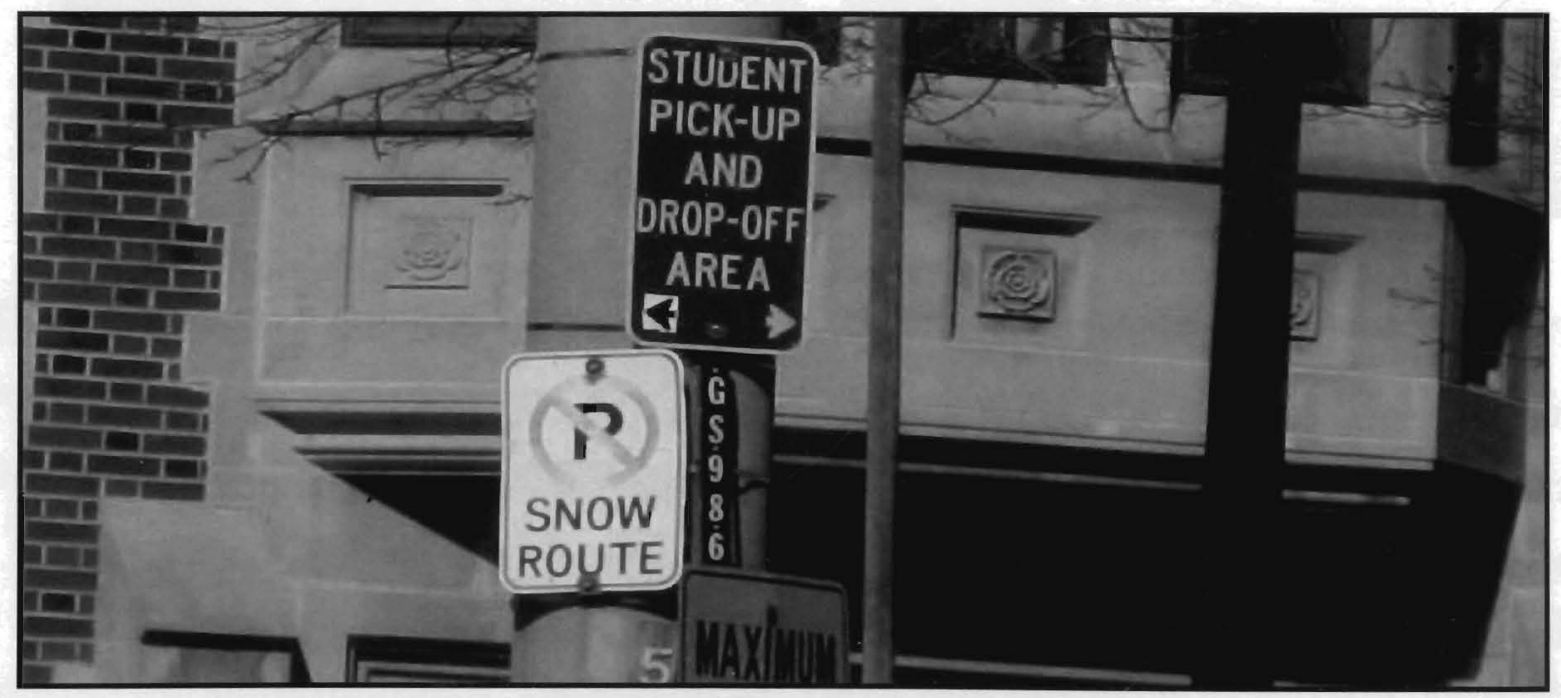

Figure 36: Student pick up \& drop-off area at Coxwell \& Cosburn 


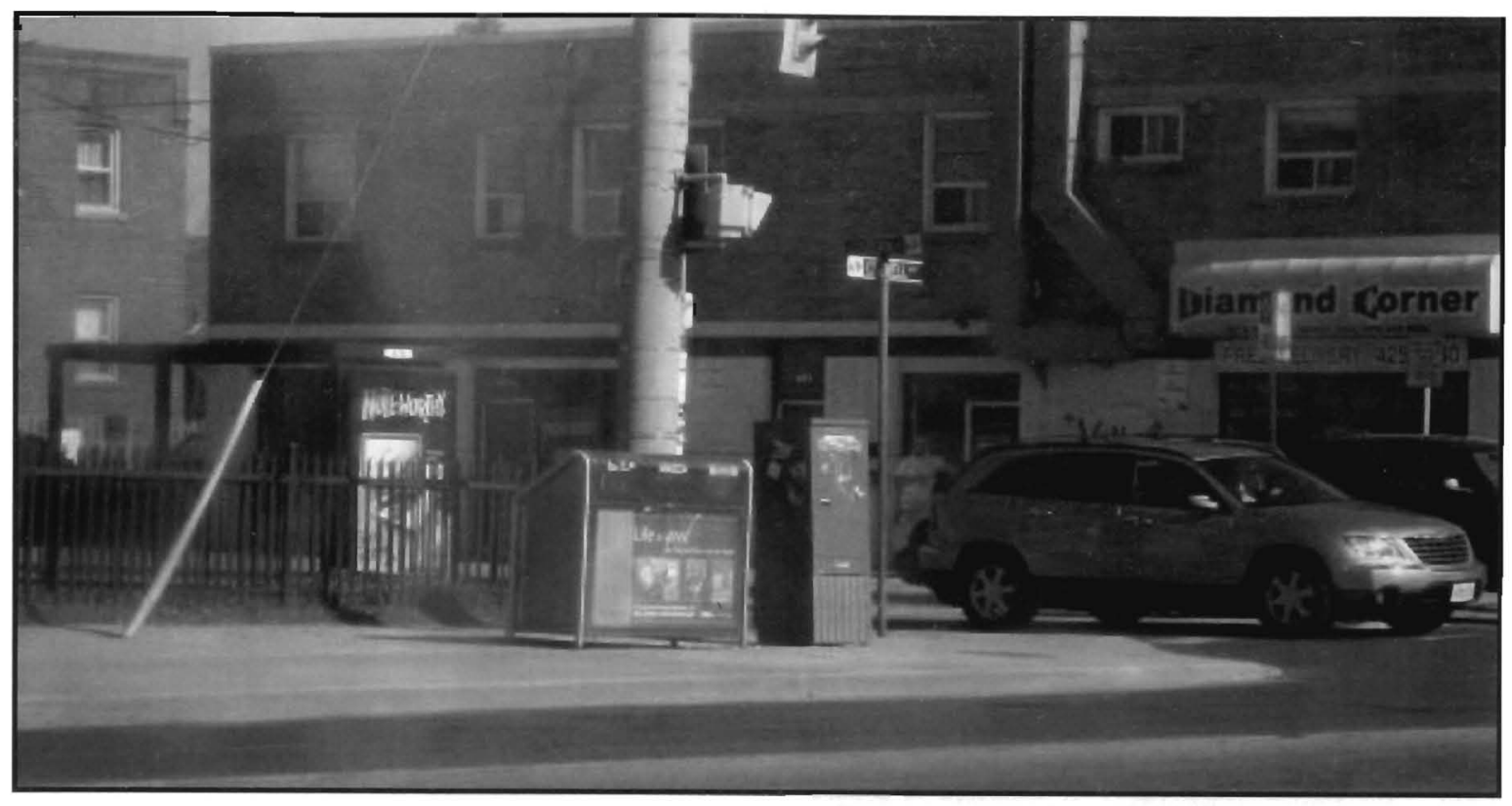

Figure 37: View of Coxwell \& Cosburn intersection

Dust bin and Canada Post mail collection box have been placed very close to the edge of pavement which block the right turning traffic's view and are therefore a potential hazard for pedestrian collisions

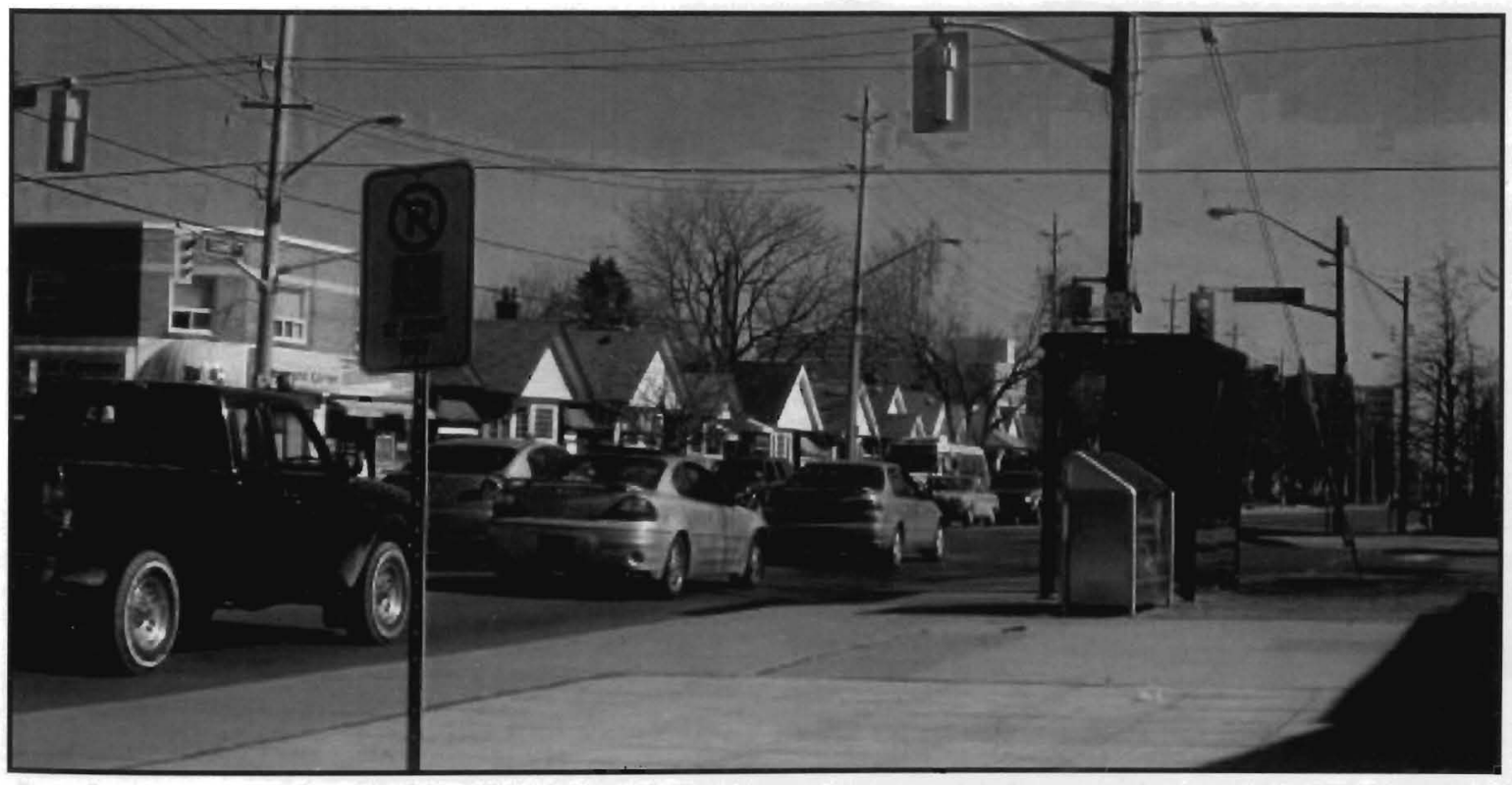

Figure 38: No bus bay provided at Coxwell \& Cosburn

Traffic queuing occurs due to the bus stop location; either the bus stop should be located further back or a bus bay should be provided to better safety and reduce delays. Plenty of space is available for bus bay provision. 


\section{Coxwell \& O'Connor:}

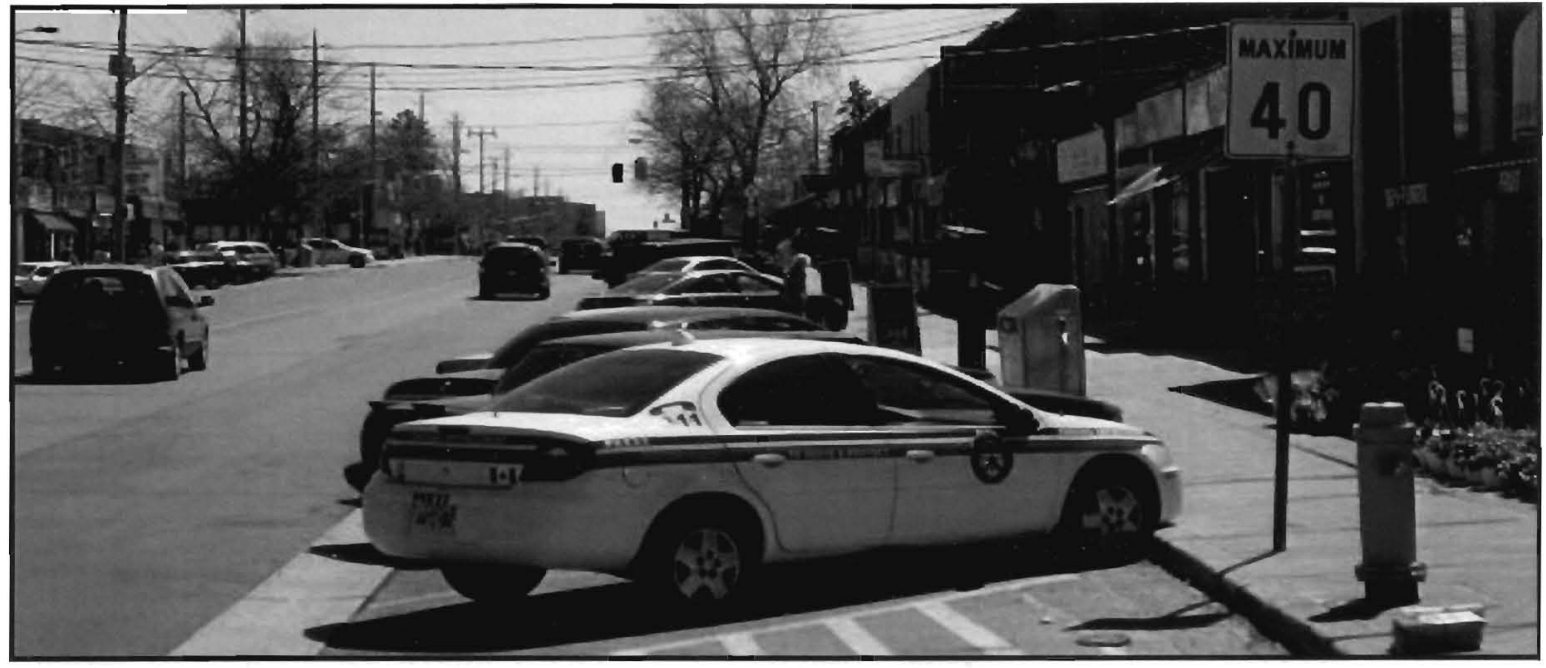

Figure 39: Speed limit sign needs relocation- Coxwell \& O'Connor

The maximum speed sign is awkwardly positioned; relocating the sign to improve visibility would help better safety for the intersection. Reverse parking is being used which should be replaced by parallel parking as the reversing vehicles block the roadway increasing delays and also increasing conflicts.

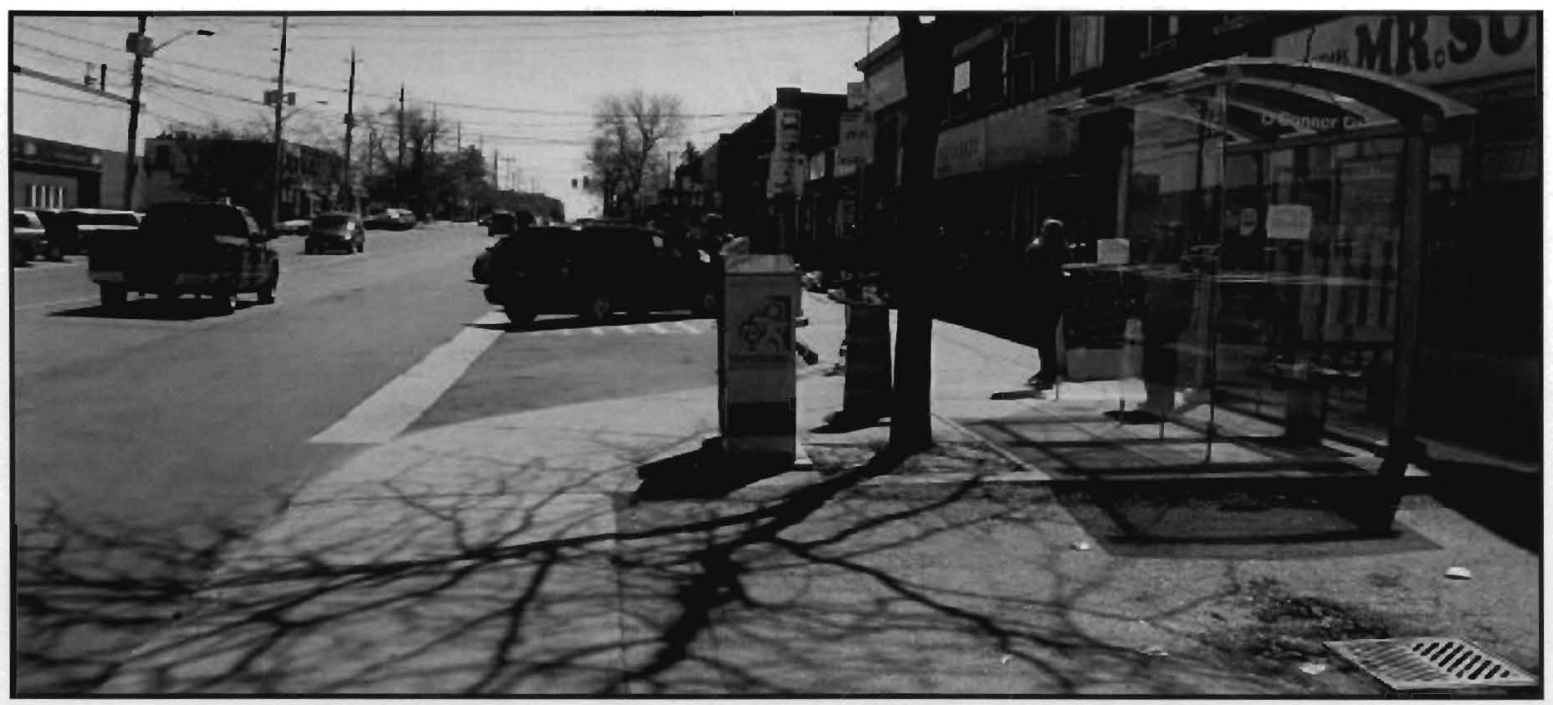

Figure 40: No bus bay at Coxwell \& O'Connor

Figure 40 also shows the available space just next to the bus stop (located south of O'Connor- Coxwell intersection) which should be ideally utilized by providing a bus bay. Due to proximity of the bus stop to the intersection, the bus bay would alleviate queuing and improve flow of traffic. 
Figure 42 shows oncoming traffic from gas station which creates conflicts and increases probability of collisions. While there is space available as shown in Figure 41, an extra center lane would have bettered safety by accommodating transition of vehicles from gas station with the street flow.

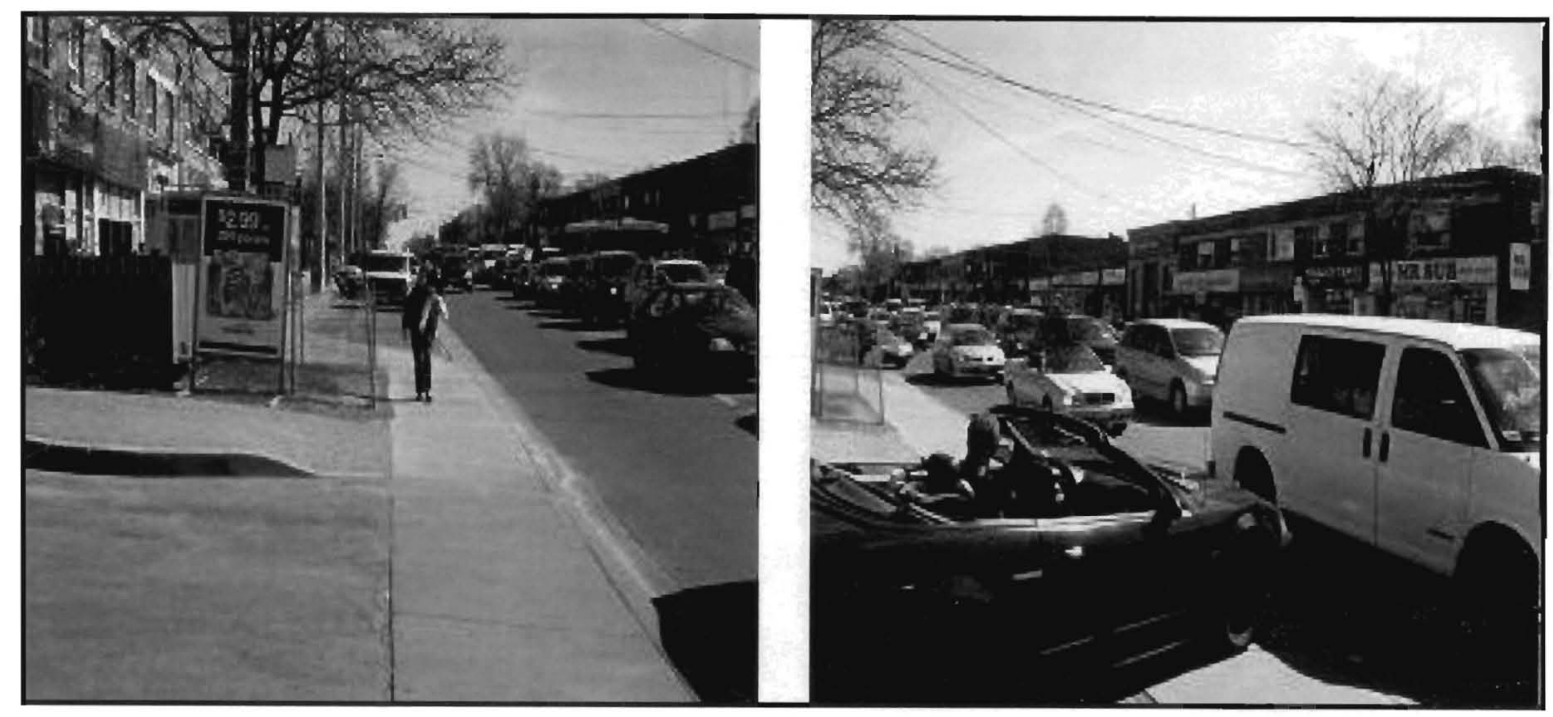

Figures $41 \& 42$ : Entry/ Exit from gas station at Coxwell \& O'Connor 


\section{O'Connor \& Don Mills:}

This is one of the major focus areas in the network. It accommodates outgoing and incoming traffic to and from the Don Valley Parkway.

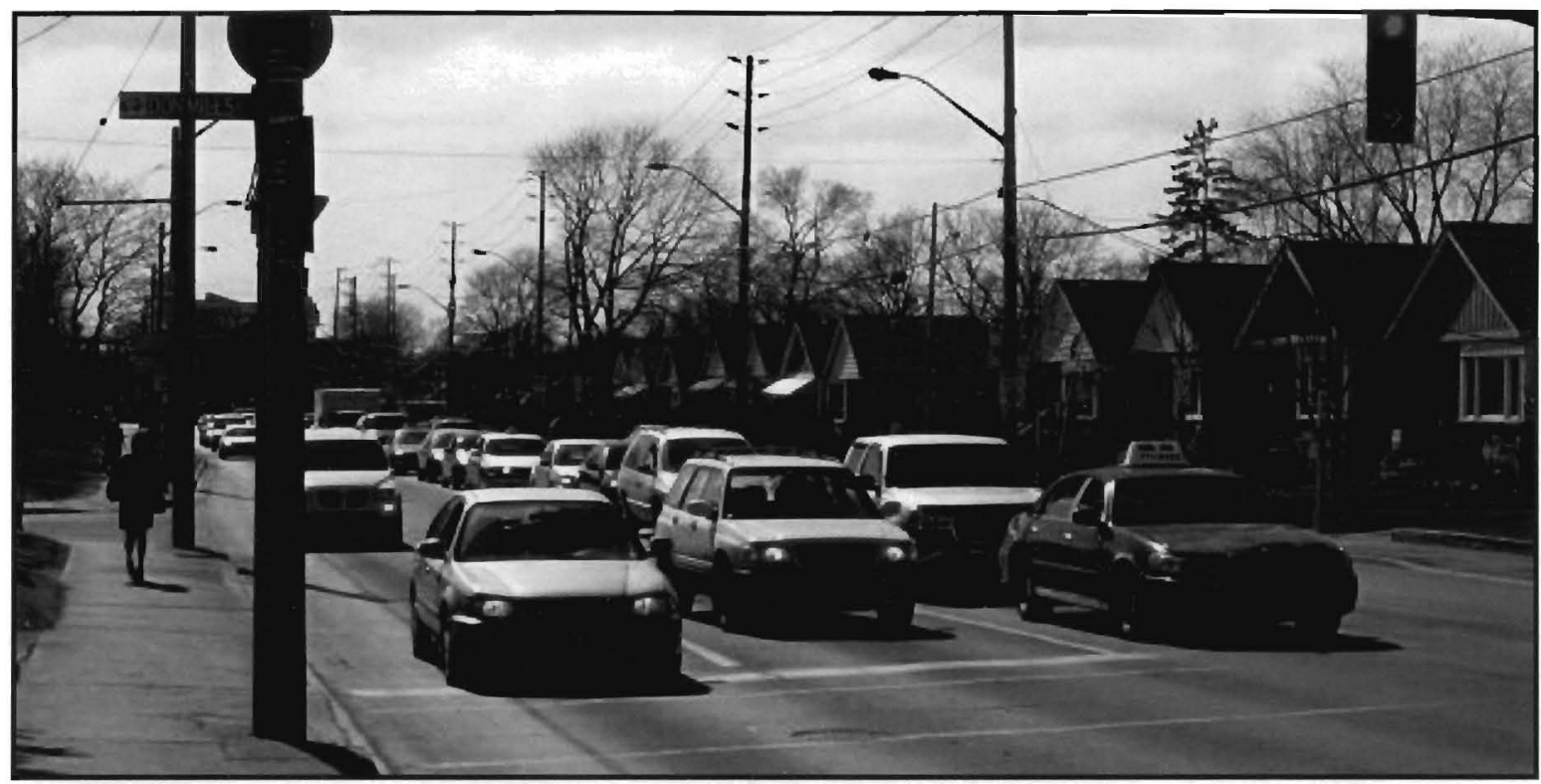

\section{Figure 43: Queues at O'Connor \& Don Mills}

As evident from the Figure 43 and 44, long queues hold traffic from getting on to and from the DVP. Queues line up as far as the adjacent intersection causing starvation delays. Figure 44 shows presence of an access point right next to the junction which causing conflicting movements.

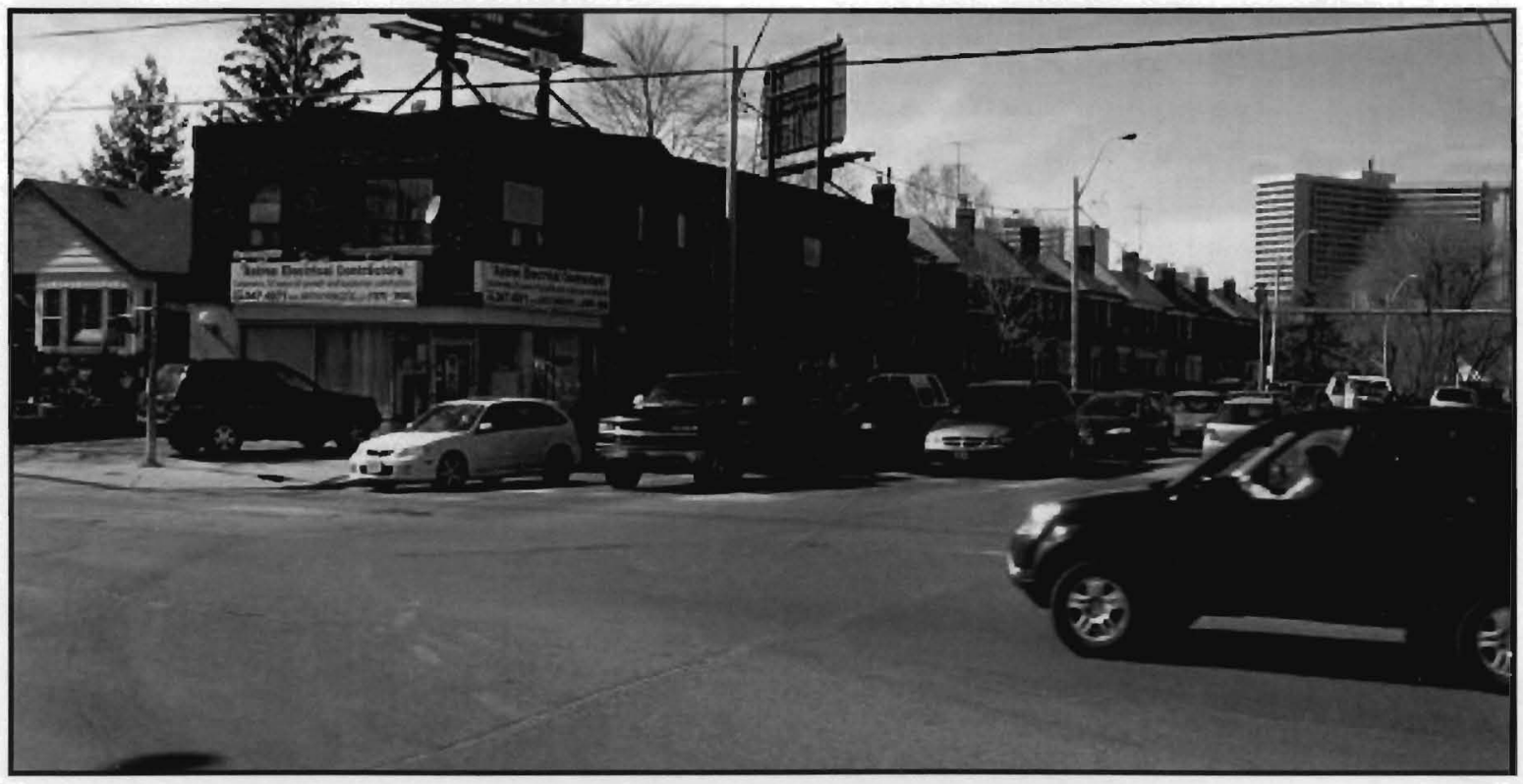

Figure 44: O’Connor \& Don Mills 


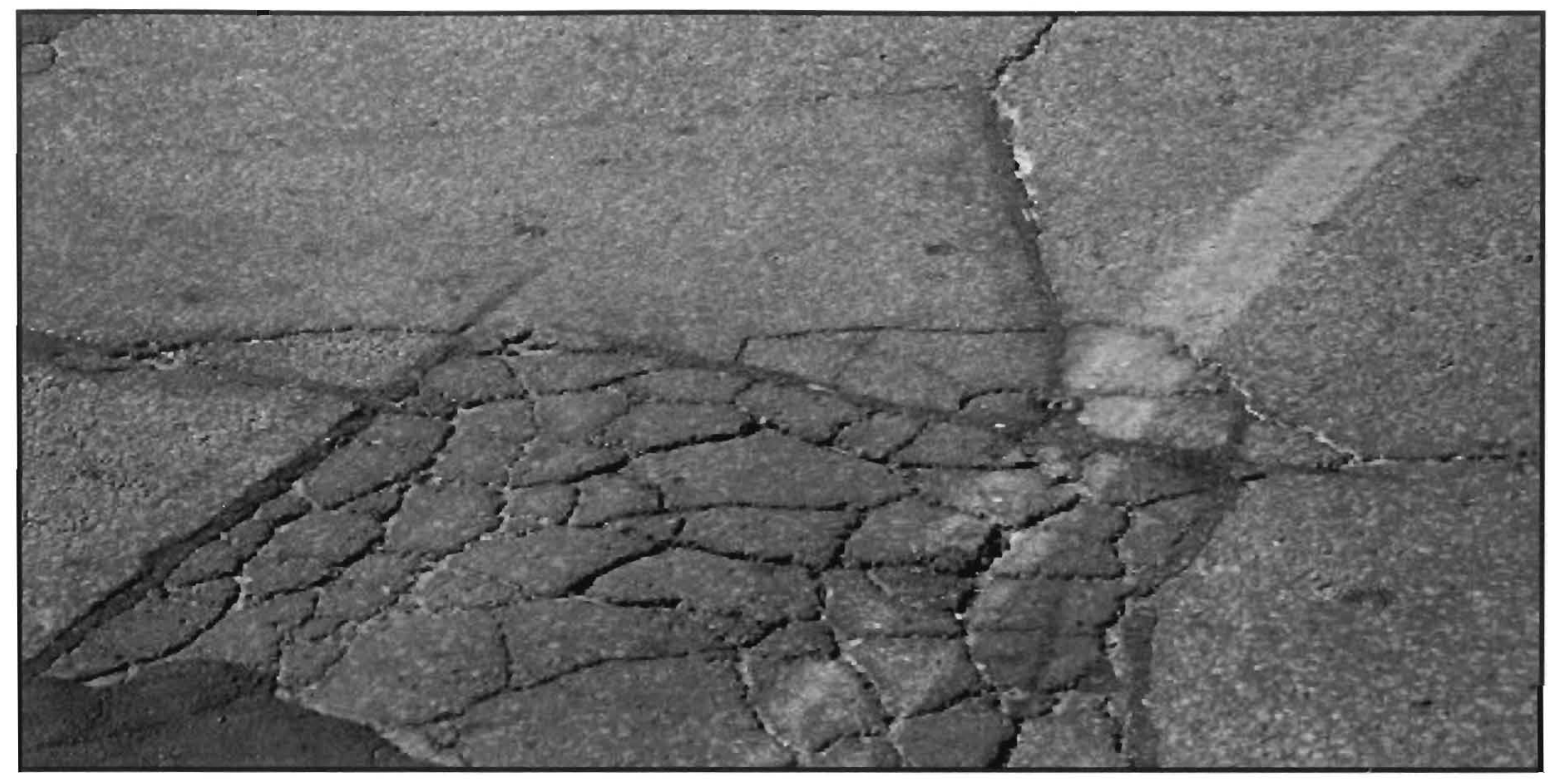

Figure 45: Pavement needs reconditioning at O’Connor \& Don Mills

Pavement quality needs to be upgraded Cracks and potholes in pavement are commonly found on approaches to the intersection. Pavement markings need to be repainted as well. The signs need to be re-located to a place where they are more visible. Also some of the signs need re-painting like the one above the driveways sign. Regulations on these signs are hardly visible. Presence of large number of driveways on intersection approach also increases chances of side swipe and angle collisions.

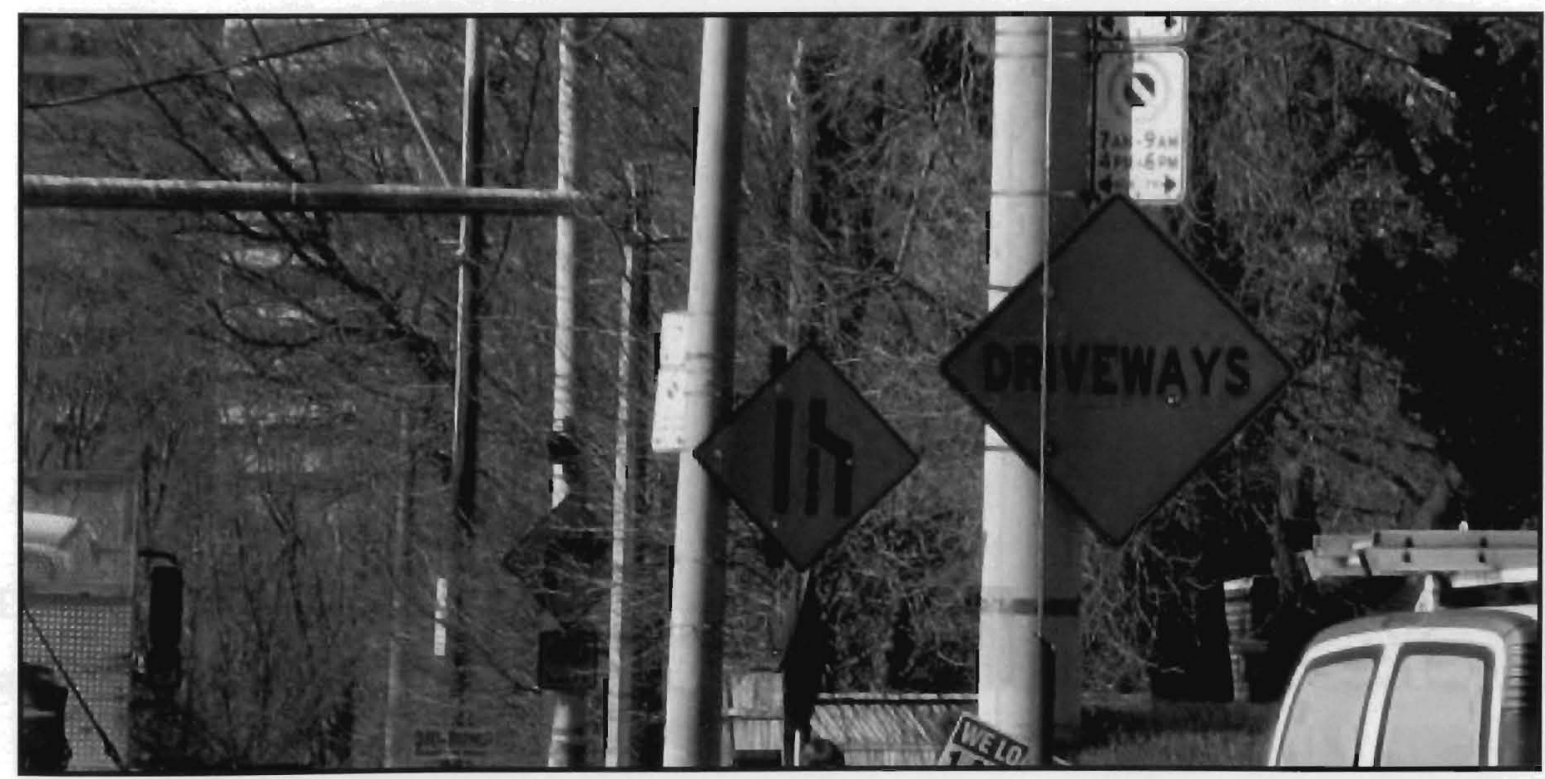

Figure 46: Signs need relocation - O’Connor \& Don Mills 


\section{O' Connor \& Donlands:}

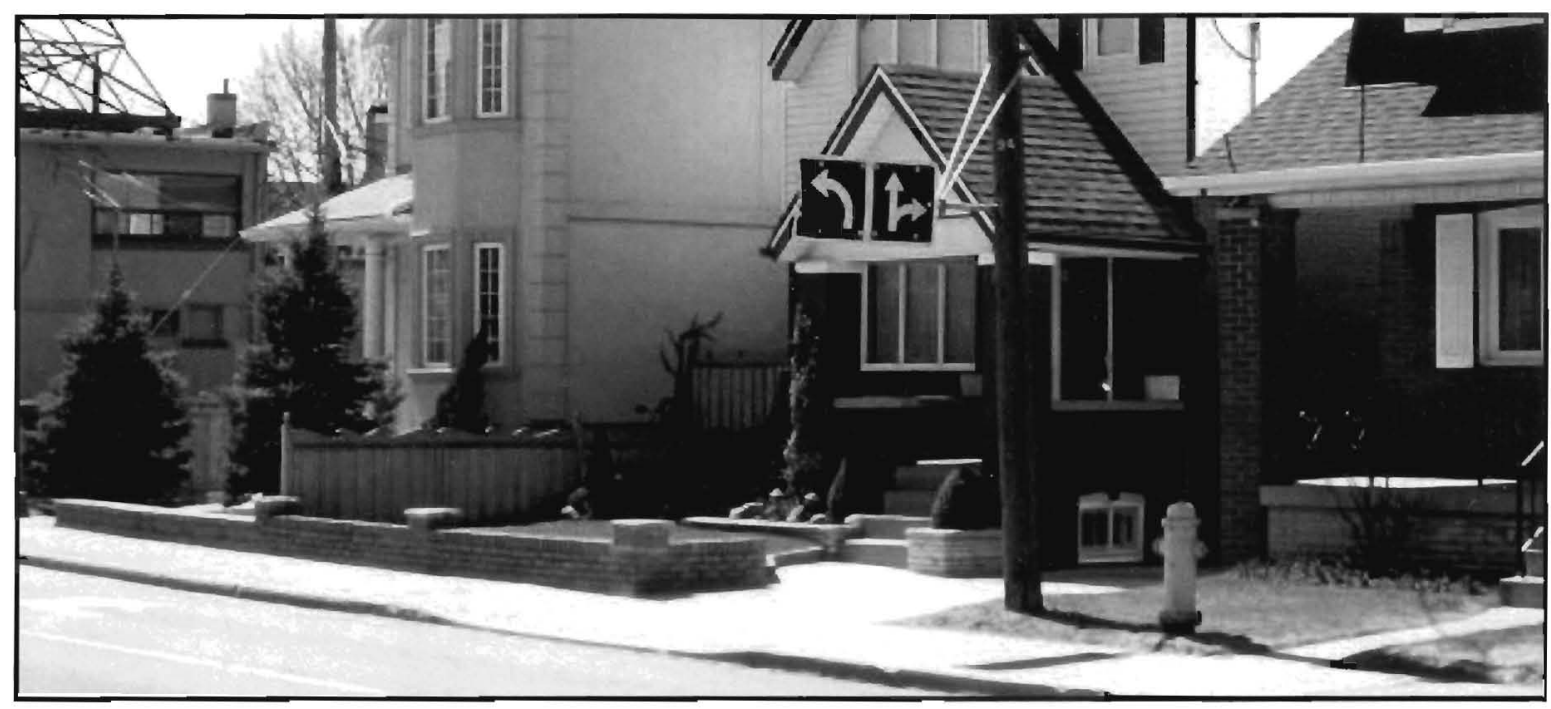

Figure 47: Signs need to be relocated - O' Connor \& Donlands

O' Connor \& Donlands recorded a total of 60 accidents in a 5 year period (2002-2006) which shows the need for a comprehensive safety analysis for the site The signs need to be relocated to a conspicuous and safe place; the direction sign due to its low height has been struck by a truck as shown in Figure 47 . Figure 47 shows existence of too many driveways close to the junction which increases probability of potential side swipe collisions. Reverse parking is in effect (Refer Figure 48); if replaced by parallel parking it would better safety. Pavement needs to be reconditioned; pot holes and cracks are plentiful.

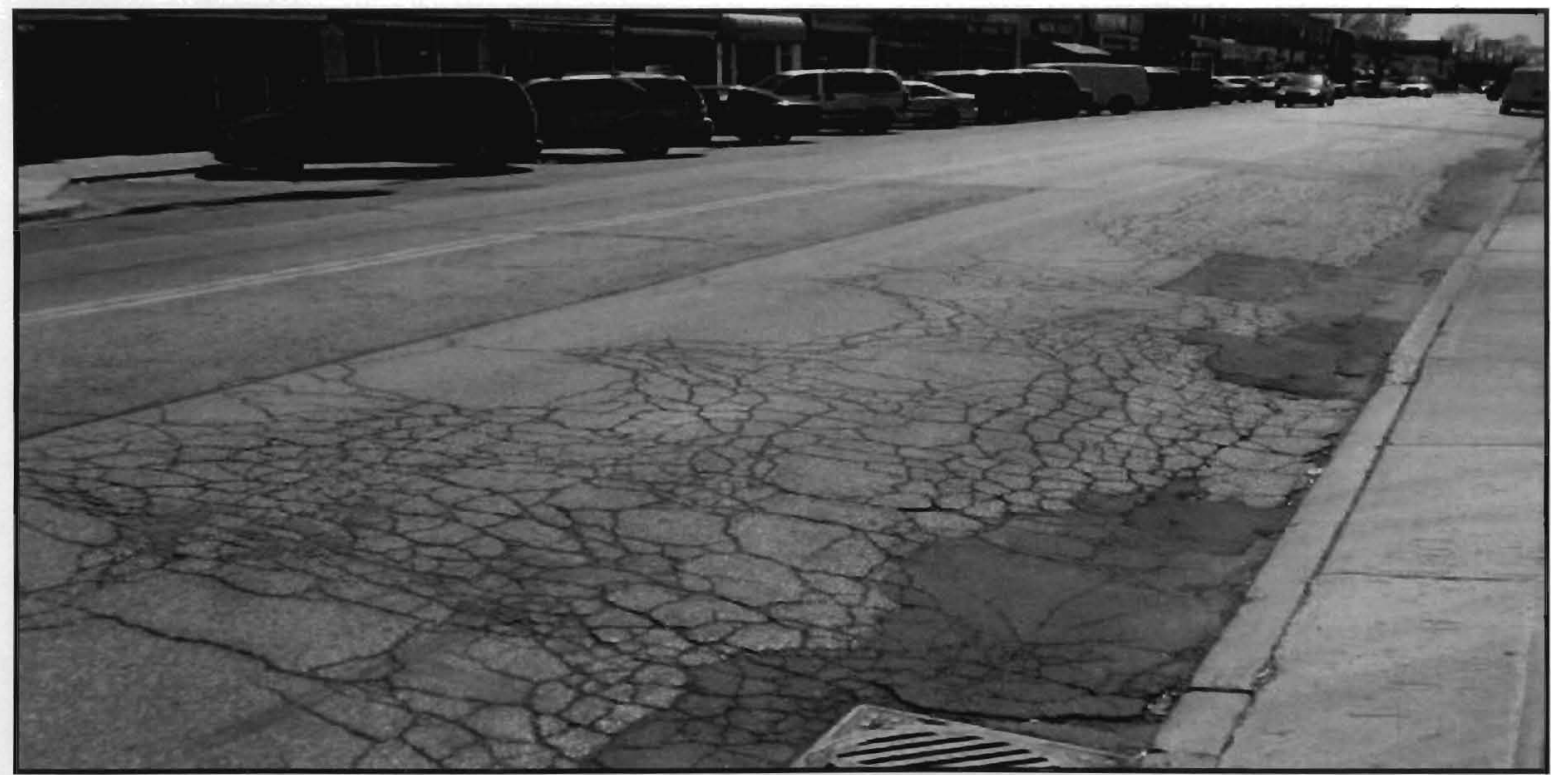

Figure 48: Pot holes and cracks at O’ Connor \& Donlands 


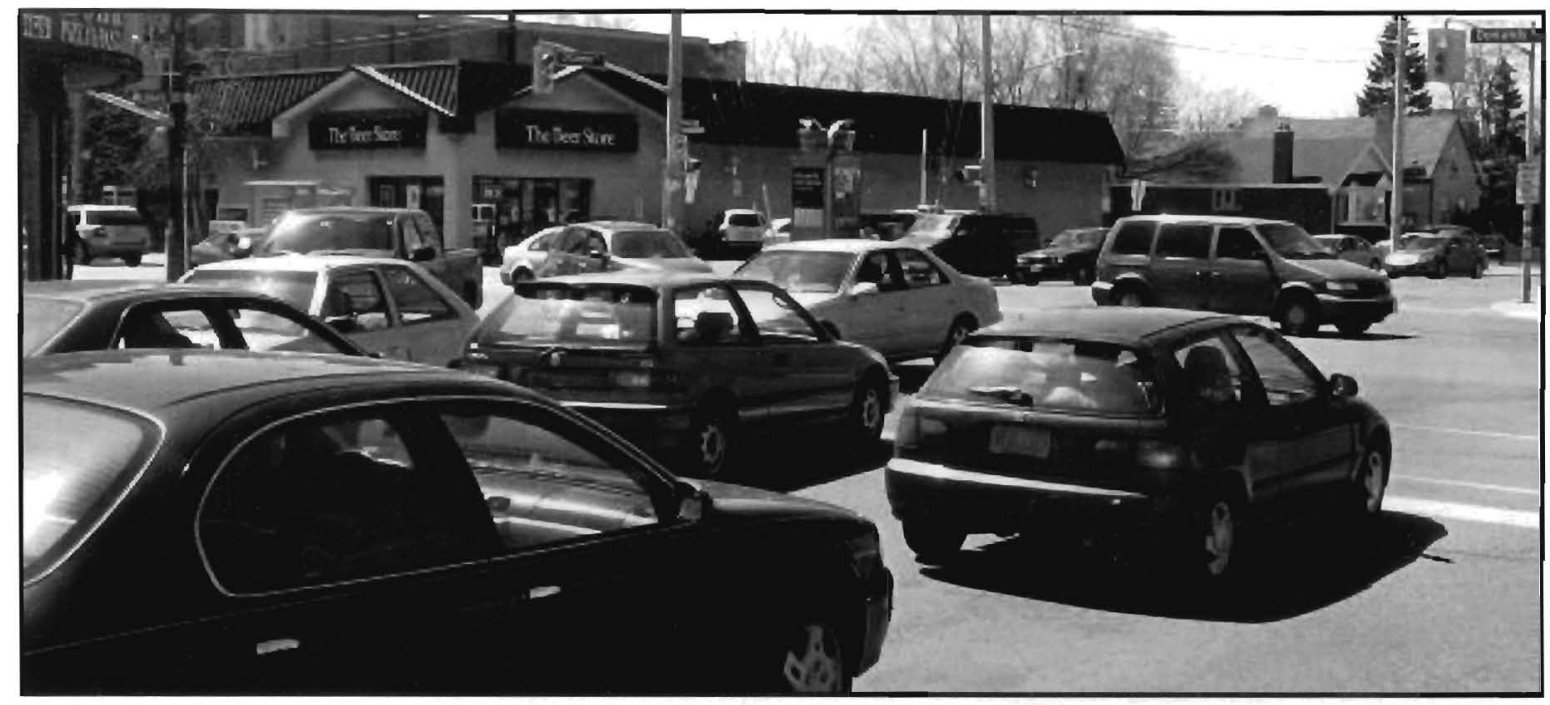

Figure 49: Congestion - O’ Connor \& Donlands

The stop line should be pulled back as the vehicles on the stop line block the turning traffic (Refer Figure 49). As many 20 turning movement accidents have been recorded at this intersection in years 2002-2006 (Refer Collision Data graphical analysis).

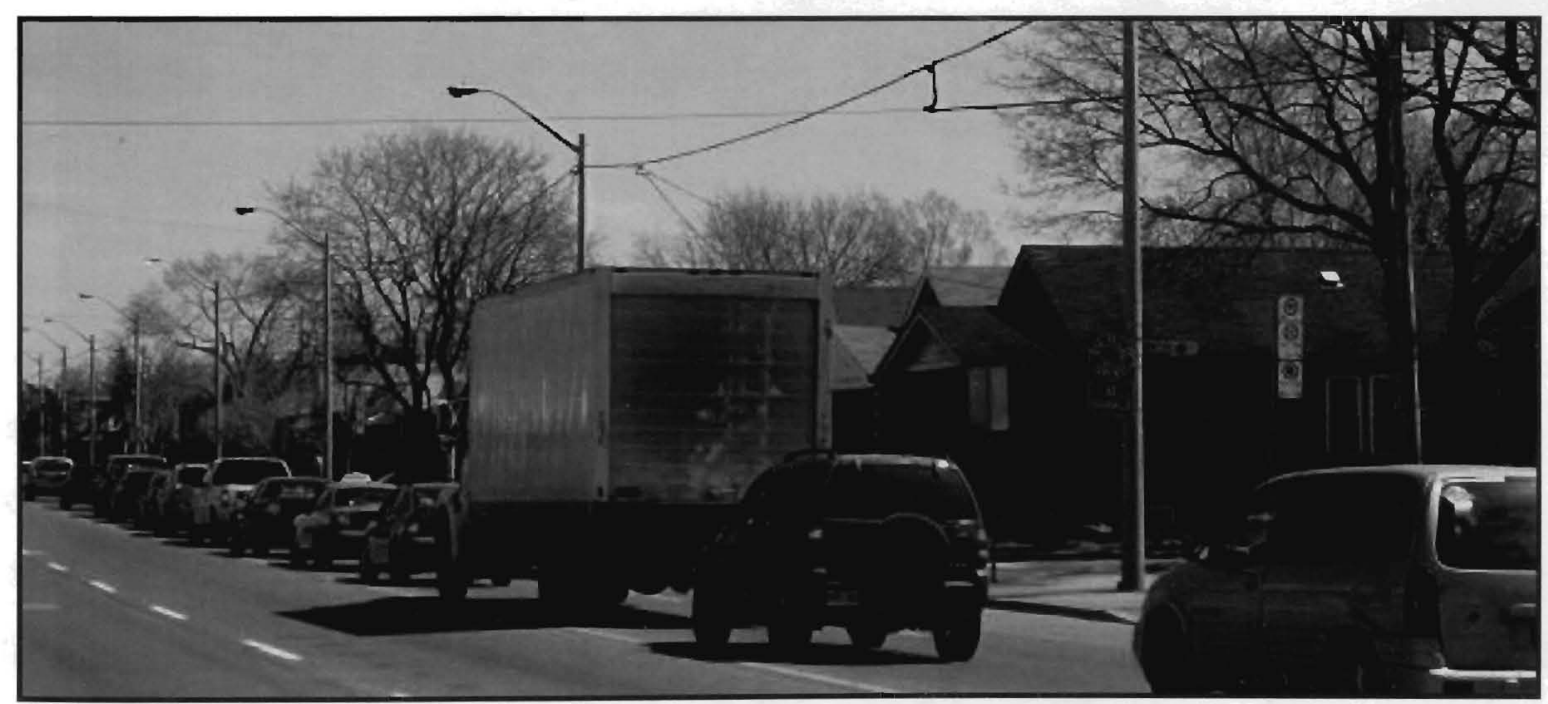

Figure 50: Long queues close to $\mathrm{O}^{\prime}$ Connor \& Donlands

Due to proximity of adjacent intersection and high volumes, starvation delays are frequent and queuing extends as far as the next intersection. 


\section{Cosburn \& Donlands:}

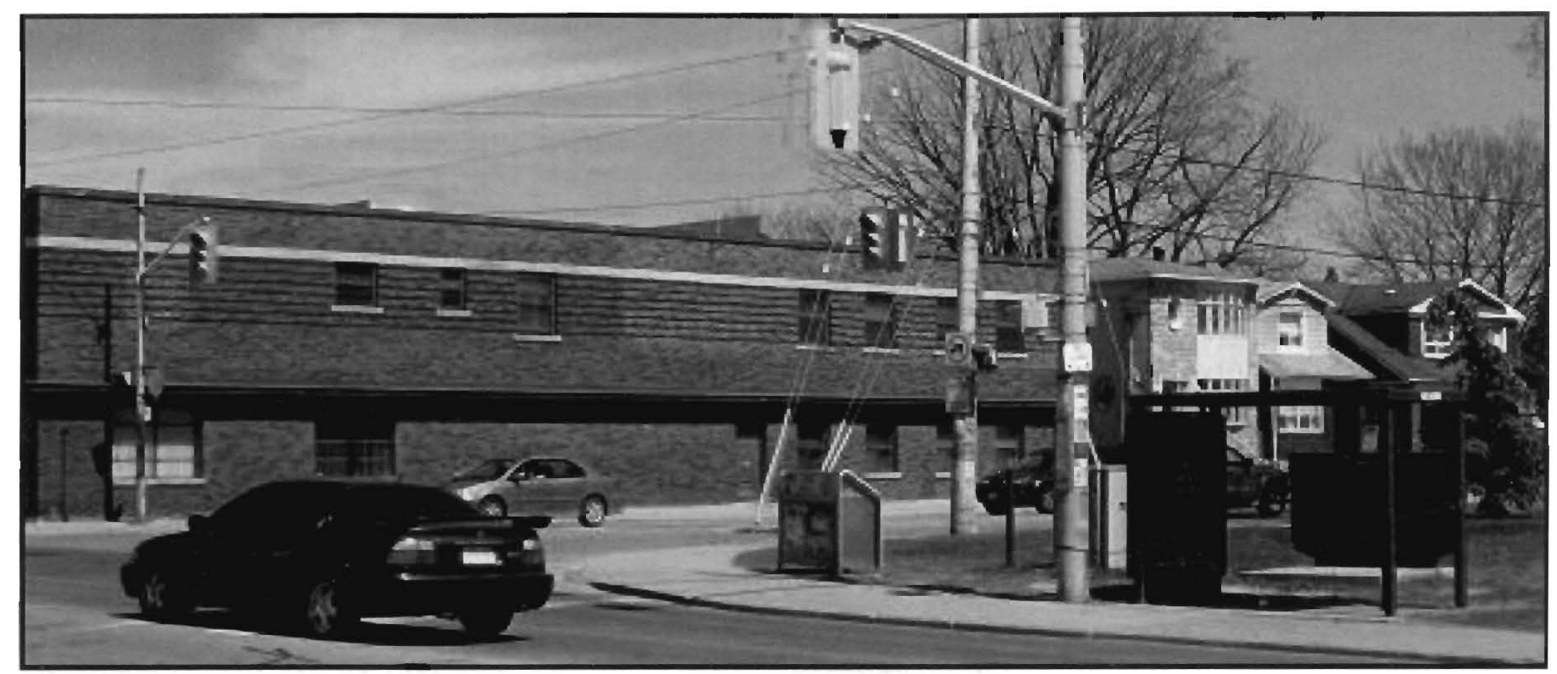

Figure 51: No bus bays at Cosburn \& Donlands

As found in many of the other intersections inspected, no bus bays have been provided here as well. The stop is very close to the junction and hence blocks traffic flow and increases delays for auto users. The electric pole and its supporting cables (yellow color) block pedestrian walkway and could be a potential hazard to pedestrian safety.

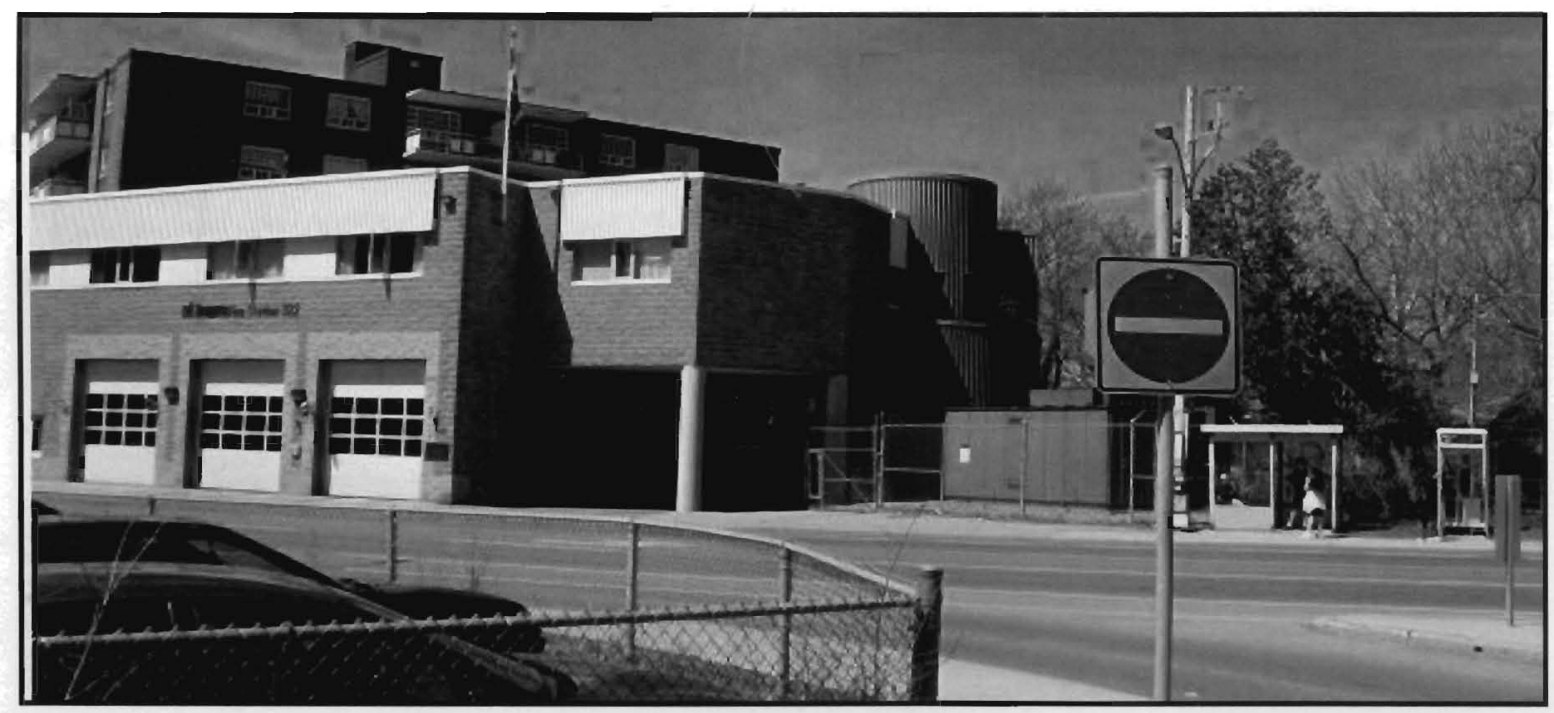

Figure 52: View of Cosburn \& Donlands intersection

As evident from Figure 52 the fire station is extended right up to the edge of pavement and there exists a bus stop very close to the access of the fire station. This poses a safety threat as it could lead to delays and congestion close to the junction. The bus stop should either be moved further away from the intersection or a bus bay should be installed so it does not block the roadway. 


\section{Conclusions}

In this study, a network of nine intersections in East York was selected for review for potential operational and safety improvement. This review was desirable because geometric and operational characteristics had become incompatible with present travel demands that have grown dramatically due to rapid growth in population, employment and land use. Simulation and optimization techniques were used to assess and improve existing level of service (LOS) for the intersections. For the safety review, the adopted approach was based on the "Road Safety Audit Guide" developed by Transportation Association of Canada (TAC). Site visits were performed to investigate lane configuration, phasing sequence and signal timing. Traffic volumes and collision data were provided by City of Toronto.

The data were analysed using Transyt and Synchro software to establish the level of service of the nine intersections in the network. Simulation and optimization in Transyt-7F revealed that, during the morning rush period, three out of the nine intersections in the network were failing with a LOS of F, while there was one intersection each with LOS of D and E, respectively. LOS of $\mathrm{F}$ was obtained for five intersections in Synchro simulation of the same data period. Although a reduction in delays was obtained through optimization, no significant change in level of service was achieved without altering lane configuration and geometry.

An analysis of collision data revealed three intersections- Coxwell Avenue, O'Connor Road and Donlands Avenue- as the most poorly performing streets in the entire network and pointed to a need for a detailed investigation of these intersections for possible safety improvement. The investigation revealed numerous safety problems and potential treatments such as relocation of traffic signs, re-timing signals, addition of bus bays, installation of active signals and replacing intersections with roundabouts. Further to these treatments, enhancing programs of law enforcement with public information and education campaigns would facilitate safety improvement in the network. 


\section{References}

1. Abbas, K., Traffic safety assessment and development of predictive models for accidents on rural roads in Egypt, 2002.

2. Al-Ghamdi, Ali S., Analysis of traffic accidents at urban intersections in Riyadh, Accident Analysis and Prevention, Pergamon, Riyadh, 2003.

3. Bauer, K., Harwood, D., Richard, R. and Hughes, W., Safety Effects of Narrow Lanes and Shoulder-Use Lanes to Increase Capacity of Urban Freeways, Transportation Research Board, 2005.

4. Case Study on roundabouts; Wilson St/Meadowbrook Dr, Hamilton, ON, http://www.roundabouts.ca/orc_highspeedroads.htm (accessed April 12, 2007).

5. Don Valley Parkway, www.wikipedia.com (accessed March 2, 2007).

6. East York, Ontario, www.wikipedia.com (accessed March 2, 2007).

7. Eccles, K., Hummer, J., Safety Effects of fluorescent yellow warning signs at hazardous sites in daylight, Transportation Research Board, 2001.

8. Federal Highway Administration, Transportation Performance Measures in Australia, Canada, Japan, and New Zealand, US Department of Transportation, Washington, D.C., 2004.

9. Kane, T., Performance Measures to Improve Transportation Systems, National Academy Press, Washington, D.C., 2005.

10. Persaud, B., Hauer, E., Retting, R., Vallurupalli, R., Mucsi, K., Crash Reductions related to Traffic Signal Removal in Philadelphia. Transportation Research Board, 1997.

11. Sudani, D., Road Safety Audit for a regional corridor, Ryerson University, 2006.

12. Synchro Studio 7 User Guide, Trafficware, 2007.

13. Traffic Analysis Software Tools, Transportation Research Board, 2000. www.nationalacademies.org/trb (accessed March 11, 2007).

14. Transportation Association of Canada, Performance Measures for Road Networks: A Survey of Canadian Use, TAC, 2006.

15. Transportation Association of Canada, The Canadian Guide to In-Service Road Safety Reviews, TAC, 2004.

16. Transyt, http://en.wikipedia.org/wiki/Transyt (accessed March 22, 2007). 
Appendices 
Appendix A

Transyt Report 
Simulation Results 
TRANSYT-7F Release 10.2 -- Node Output Summary (Detailed) SIMULATION RESULTS

Node Number: 1

Output Flow (vph)

Degree of Sat. ( $(z)$

Tot. Travel (veh-km)

Tot.TravTime (veh-h)

Avg.TravTime (sec/v)

Unif. Delay (veh-h)

Rand. Delay (veh-h)

Total Delay (veh-h)

Avg. Delay (sec/v)

Unif. Stops (vph)

Unif. Stops (8)

Rand. Stops (vph)

Rand. Stops (8)

Total Stops (vph)

Total Stops (8)

Unif. MBOQ (veh)

Unif. $M B O Q$ ( $m /$ lane)

Rand. MBOQ (veh)

Rand. $M B O Q$ ( $m /$ lane)

Total MBOQ (veh)

Total MBOQ ( $\mathrm{m} / \mathrm{lane}$ )

Q. Capacity (veh)

Q.Capacity (m/lane)

Time Full ( 8$)$

Critical Link ( $Y / N)$

Fuel Consumpt. (lit)

EffectiveGreen (sec)

Arrival Type (1-6)

Level of Service

\begin{tabular}{|c|c|c|c|c|c|c|c|c|c|}
\hline \multicolumn{2}{|c|}{ Eastbound } & \multicolumn{3}{|c|}{ Westbound } & \multicolumn{2}{|c|}{ Northbound } & \multicolumn{3}{|c|}{ Southbound } \\
\hline TH & RT & $\mathrm{LT}$ & $\mathrm{TH}$ & $\mathrm{RT}$ & LT & RT & LT & TH & $\mathrm{RT}$ \\
\hline 856 & 107 & 32 & 274 & & 591 & & 73 & 437 & \\
\hline 107 & 12 & 40 & 27 & & 46 & & 32 & 67 & \\
\hline 674 & 80 & 12 & 110 & & 237 & & 41 & 238 & \\
\hline 28.8 & 2.1 & 0.5 & 3.1 & & 8.4 & & 1.7 & 9.8 & \\
\hline 121 & 71 & 62 & 41 & & 51 & & 87 & 80 & \\
\hline 4.3 & 0.5 & 0.1 & 0.9 & & 3.4 & & 0.8 & 4.3 & \\
\hline 10.9 & 0.0 & 0.1 & 0.0 & & 0.1 & & 0.0 & 0.6 & \\
\hline 15.3 & 0.5 & 0.2 & 0.9 & & 3.6 & & 0.9 & 5.0 & \\
\hline 64 & 17 & $: 33$ & 12 & & 22 & & 46 & 41 & \\
\hline 632 & 72 & 27 & 135 & & 401 & & 62 & 379 & \\
\hline 74 & 68 & 85 & 50 & & 68 & & 85 & 87 & \\
\hline 330 & 1 & 10 & 6 & & 13 & & 7 & 31 & \\
\hline 39 & 2 & 33 & 3 & & 3 & & 10 & 8 & \\
\hline 963 & 73 & 37 & 141 & & 415 & & 69 & 410 & \\
\hline 113 & 69 & 118 & 52 & & 71 & & 96 & 94 & \\
\hline 13.9 & 2.1 & 0.4 & 3.7 & & 10.7 & & 1.7 & 10.2 & \\
\hline 107 & 15 & 0 & 30 & & 42 & & 15 & 76 & \\
\hline 10.2 & 0.0 & 0.3 & 0.2 & & 0.4 & & 0.2 & 1.0 & \\
\hline 78 & 0 & 2 & 1 & & 2 & & 2 & 7 & \\
\hline 24.1 & 2.1 & 0.7 & 3.9 & & 11.1 & & 1.9 & 11.2 & \\
\hline 185 & 15 & 2 & 31 & & 44 & & 17 & 83 & \\
\hline 93.0 & 3.0 & 3.0 & 53.0 & & 106.0 & & 3.0 & 49.0 & \\
\hline 709 & 23 & 23 & 404 & & 404 & & 23 & 373 & \\
\hline 0.0 & 0.0 & 0.0 & 0.0 & & 0.0 & & 0.0 & 0.0 & \\
\hline $\mathrm{N}$ & $\mathrm{N}$ & $\mathrm{N}$ & $\mathrm{N}$ & & $\mathrm{N}$ & & $\mathrm{N}$ & $\mathrm{N}$ & \\
\hline 120 & 10 & 2 & 16 & & 41 & & 7 & 45 & \\
\hline 55.0 & 55.0 & 55.0 & 55.0 & & 35.0 & & 35.0 & 35.0 & \\
\hline 1 & 2 & 3 & 3 & & 3 & & 1 & 1 & \\
\hline $\mathrm{E}$ & B & $\mathrm{C}$ & B & & C & & D & D & \\
\hline
\end{tabular}

Overall Intersection Results

Output Flow (vph) 2370

Degree of Sat. (8) 107

Tot. Travel (veh-km) 1395

Tot.TravTime (veh-h) 54

Unif. Delay (veh-h) 14.7

Rand. Delay $(v e h-h) 12.0$

Total Delay (veh-h) 26.7

Avg. Delay (sec/v) 40

Unif. Stops (vph) 1710

Unif. Stops (8) 72

Rand. Stops (vph) 400

Rand. Stops (8) 17

Total Stops (vph) 2111

Total Stops $(8) \quad 89$

Time Full (8) 0

Fuel Consumpt. (lit) 245

Disutility Index 32

Level of Service 
Node Number: 2

Output Flow (vph)

Degree of Sat. (z)

Tot. Travel (veh-km)

Tot.TravTime (veh-h)

Avg.TravTime (sec/v)

Unif. Delay (veh-h)

Rand. Delay (veh-h)

Total Delay (veh-h)

Avg. Delay (sec/v)

Unif. Stops (vph)

Unif. Stops (8)

Rand. Stops (vph)

Rand. Stops $(z)$

Total Stops (vph)

Total Stops (q)

Unif. MBOQ (veh)

Unif. MBOQ (m/lane)

Rand. MBOQ (veh)

Rand. $M B O Q$ ( $m /$ lane)

Total MBOQ (veh)

Total MBOQ (m/lane)

Q.Capacity (veh)

Q. Capacity (m/lane)

Time Full (z)

Critical Link ( $Y / N)$

Fuel Consumpt. (1it)

EffectiveGreen (sec)

Arrival Type (1-6)

Level of Service

\begin{tabular}{|c|c|c|c|c|c|c|c|c|c|c|}
\hline \multicolumn{2}{|c|}{ Eastbound } & \multicolumn{3}{|c|}{ Westbound } & \multicolumn{3}{|c|}{ Northbound } & \multicolumn{3}{|c|}{ Southbound } \\
\hline $\mathrm{TH}$ & $\mathrm{RT}$ & LT & $\mathrm{TH}$ & $\mathrm{RT}$ & $\mathrm{LT}$ & $\mathrm{TH}$ & RT & LT & $\mathrm{TH}$ & RT \\
\hline 331 & & 40 & 253 & & & 651 & & & 587 & \\
\hline 45 & & 19 & 55 & & & 57 & & & 31 & \\
\hline 133 & & 16 & 101 & & & 270 & & & 164 & \\
\hline 5.3 & & 0.7 & 4.6 & & & 6.3 & & & 3.8 & \\
\hline 57 & & 65 & 66 & & & 34 & & & 23 & \\
\hline 2.4 & & 0.3 & 2.2 & & & 0.5 & & & 0.5 & \\
\hline 0.1 & & 0.0 & 0.3 & & & 0.3 & & & 0.0 & \\
\hline 2.6 & & 0.4 & 2.6 & & & 0.8 & & & 0.5 & \\
\hline 28 & & 36 & 37 & & & 4 & & & 3 & \\
\hline 246 & & 32 & 211 & & & 119 & & & 96 & \\
\hline 75 & & 81 & 84 & & & 18 & & & 16 & \\
\hline 13 & & 3 & 19 & & & 17 & & & 7 & \\
\hline 4 & & 10 & 8 & & & 3 & & & 2 & \\
\hline 259 & & 36 & 230 & & & 136 & & & 103 & \\
\hline 79 & & 91 & 92 & & & 22 & & & 18 & \\
\hline 6.7 & & 0.9 & 5.8 & & & 3.3 & & & 2.7 & \\
\hline 27 & & 8 & 46 & & & 23 & & & 11 & \\
\hline 0.4 & & 0.1 & 0.6 & & & 0.5 & & & 0.2 & \\
\hline 2 & & 1 & 5 & & & 4 & & & 1 & \\
\hline 7.1 & & 1.0 & 6.4 & & & 3.9 & & & 2.9 & \\
\hline 29 & & 9 & 51 & & & 27 & & & 12 & \\
\hline 106.0 & & 3.0 & 52.0 & & & 53.0 & & & 54.0 & \\
\hline 404 & & 23 & 396 & & & 404 & & & 206 & \\
\hline 0.0 & & 0.0 & 0.0 & & & 0.0 & & & 0.0 & \\
\hline $\mathrm{N}$ & & $\mathrm{N}$ & $\mathrm{N}$ & & & $N$ & & & $\mathrm{~N}$ & \\
\hline 25 & & 3 & 21 & & & 30 & & & 19 & \\
\hline 25.0 & & 25.0 & 25.0 & & & 65.0 & & & 65.0 & \\
\hline 3 & & 3 & 3 & & & 1 & & & 1 & \\
\hline $\mathrm{C}$ & & D & D & & & $A$ & & & A & \\
\hline
\end{tabular}

Overall Intersection Results

$\begin{array}{lr}\text { Output Flow (vph) } & 1862 \\ \text { Degree of Sat. (8) } & 57 \\ \text { Tot. Travel (veh-km) } & 685 \\ \text { Tot.TravTime (veh-h) } & 20 \\ \text { Unif. Delay (veh-h) } & 6.2 \\ \text { Rand. Delay (veh-h) } & 0.9 \\ \text { Total Delay (veh-h) } & 7.1 \\ \text { Avg. Delay (sec/v) } & 13 \\ \text { Unif. Stops (vph) } & 706 \\ \text { Unif. Stops (8) } & 38 \\ \text { Rand. Stops (vph) } & 60 \\ \text { Rand. Stops (8) } & 3 \\ \text { Total Stops (vph) } & 767 \\ \text { Total Stops (8) } & 41 \\ \text { Time Eull (8) } & 0 \\ \text { Fuel Consumpt. (lit) } & 100 \\ \text { Disutility Index } & 9 \\ \text { Level of Service } & \text { B }\end{array}$


Node Number: 3

Output Flow (vph)

Degree of Sat. (\%)

Tot. Travel (veh-km)

Tot.TravTime (veh-h)

Avg. TravTime $(\mathrm{sec} / \mathrm{v})$

Unif. Delay (veh-h)

Rand. Delay (veh-h)

Total Delay $(v e h-h)$

Avg. Delay (sec/v)

Unif. Stops (vph)

Unif. Stops (8)

Rand. Stops (vph)

Rand. Stops ( 8 )

Total Stops (vph)

Total Stops ( 8 )

Unif. MBOQ (veh)

Unif. MBOQ ( $\mathrm{m} / \mathrm{lane}$ )

Rand. MBOQ (veh)

Rand. MBOQ (m/lane)

Total MBOQ (veh)

Total MBOQ (m/lane)

Q.Capacity (veh)

Q. Capacity (m/lane)

Time Full ( 8$)$

Critical Link $(Y / N)$

Fuel Consumpt. (lit)

EffectiveGreen (sec)

Arrival Type (1-6)

Level of Service

\begin{tabular}{|c|c|c|c|c|c|c|c|c|c|c|}
\hline \multicolumn{2}{|c|}{ Eastbound } & \multicolumn{3}{|c|}{ Westbound } & \multicolumn{3}{|c|}{ Northbound } & \multicolumn{3}{|c|}{ Southbound } \\
\hline IT & $\mathrm{RT}$ & LT & $T H$ & $\mathrm{RT}$ & $\mathrm{LT}$ & $\mathrm{TH}$ & RT & LT & $\mathrm{TH}$ & $\mathrm{RT}$ \\
\hline 251 & & & 164 & . & & 650 & & & 470 & \\
\hline 59 & & & 42 & & & 41 & & & 22 & \\
\hline 100 & & & 65 & & & 133 & & & 157 & \\
\hline 3.9 & & & 2.3 & & & 4.5 & & & 3.9 & \\
\hline 56 & & & 51 & & & 24 & & & 30 & \\
\hline 1.4 & & & 0.8 & & & 1.7 & & & 0.8 & \\
\hline 0.4 & & & 0.1 & & & 0.1 & & & 0.0 & \\
\hline 1.8 & & & 1.0 & & & 1.8 & & & 0.8 & \\
\hline 27 & & : & 22 & & & 10 & & & 6 & \\
\hline 172 & & & 104 & & & 254 & & & 248 & \\
\hline 69 & & & 64 & & & 39 & & & 53 & \\
\hline 22 & & & 11 & & & 9 & & & 1 & \\
\hline 9 & & & 7 & & & 2 & & & 1 & \\
\hline 194 & & & 116 & & & 263 & & & 249 & \\
\hline 78 & & & 71 & & & 41 & & & 54 & \\
\hline 4.4 & & & 2.8 & & & 6.5 & & & 7.0 & \\
\hline 30 & & & 23 & & & 27 & & & 27 & \\
\hline 0.7 & & & 0.4 & & & 0.3 & & & 0.1 & \\
\hline 5 & & & 3 & & & 1 & & & 0 & \\
\hline 5.1 & & & 3.1 & & & 6.8 & & & 7.0 & \\
\hline 35 & & & 26 & & & 28 & & & 27 & \\
\hline 53.0 & & & 53.0 & & & 53.0 & & & 56.0 & \\
\hline 404 & & & 404 & & & 202 & & & 213 & \\
\hline 0.0 & & & 0.0 & & & 0.0 & & & 0.0 & \\
\hline $\mathrm{N}$ & & & $N$ & & & $\mathrm{~N}$ & & & $\mathrm{~N}$ & \\
\hline 18 & & & 11 & & & 23 & & & 22 & \\
\hline 36.0 & & & 36.0 & & & 54.0 & & & 54.0 & \\
\hline 3 & & & 3 & & & 1 & & & 1 & \\
\hline C & & & $\mathrm{C}$ & & & B & & & A & \\
\hline
\end{tabular}

Overall Intersection Results

$\begin{array}{lr}\text { Output Flow (vph) } & 1535 \\ \text { Degree of Sat. (8) } & 59 \\ \text { Tot. Travel (veh-km) } & 457 \\ \text { Tot.TravTime (veh-h) } & 14 \\ \text { Unif. Delay (veh-h) } & 4.9 \\ \text { Rand. Delay (veh-h) } & 0.6 \\ \text { Total Delay (veh-h) } & 5.6 \\ \text { Avg. Delay (sec/v) } & 13 \\ \text { Unif. Stops (vph) } & 779 \\ \text { Unif. Stops (8) } & 51 \\ \text { Rand. Stops (vph) } & 44 \\ \text { Rand. Stops (8) } & 3 \\ \text { Total Stops (vph) } & 824 \\ \text { Total Stops (8) } & 54 \\ \text { Time Full (8) } & 0 \\ \text { Fuel Consumpt. (lit) } & 76 \\ \text { Disutility Index } & 9 \\ \text { Level of Service } & \text { B }\end{array}$ 
Node Number: 4

Output Flow (vph)

Degree of Sat. (z)

Tot. Travel (veh-km)

Tot.TravTime (veh-h)

Avg.TravTime ( $\mathrm{sec} / \mathrm{v}$ )

Unif. Delay (veh-h)

Rand. Delay (veh-h)

Total Delay (veh-h)

Avg. Delay (sec/v)

Unif. Stops (vph)

Unif. Stops (8)

Rand. Stops (vph)

Rand. Stops (\&)

Total Stops (vph)

Total Stops (\&)

Unif. MBOQ (veh)

Unif. $M B O Q$ ( $m /$ lane)

Rand. $M B O Q$ (veh)

Rand. $M B O Q$ ( $m /$ lane)

Total MBOQ (veh)

Total MBOQ (m/lane)

Q. Capacity (veh)

Q. Capacity (m/lane)

Time Full (z)

Critical Link $(Y / N)$

Fuel Consumpt. (1it)

EffectiveGreen (sec)

Arrival Type $(1-6)$

Level of Service

\begin{tabular}{|c|c|c|c|c|c|}
\hline \multicolumn{2}{|c|}{ Eastbound } & \multicolumn{2}{|c|}{ Westbound } & \multicolumn{2}{|c|}{ Northbound } \\
\hline TH & $\mathrm{RT}$ & $\mathrm{TH}$ & $\mathrm{RT}$ & $\mathrm{TH}$ & $\mathrm{RT}$ \\
\hline 2176 & & 1067 & & 611 & \\
\hline 85 & & 94 & & 196 & \\
\hline 1967 & & 428 & & 134 & \\
\hline 47.0 & & 14.9 & & 92.7 & \\
\hline 77 & & 50 & & 546 & \\
\hline 6.8 & & 1.6 & & 14.5 & \\
\hline 0.8 & & 4.7 & & 75.4 & \\
\hline 7.6 & & 6.3 & & 90.0 & \\
\hline 12 & & 21 & & 530 & \\
\hline 1836 & & 455 & & 611 & \\
\hline 84 & & 43 & & 100 & \\
\hline 33 & & 163 & & 1000 & \\
\hline 2 & & 16 & & 164 & \\
\hline 1869 & & 619 & & 1611 & \\
\hline 86 & & 59 & & 264 & \\
\hline 31.5 & & 7.6 & & 16.5 & \\
\hline 122 & & 30 & & 65 & \\
\hline 1.0 & & 5.0 & & 38.4 & \\
\hline 4 & & 19 & & 146 & \\
\hline 32.5 & & 12.7 & & 54.9 & \\
\hline 126 & & 49 & & 211 & \\
\hline 221.0 & & 105.0 & & 56.0 & \\
\hline 842 & & 400 & & 213 & \\
\hline 0.0 & & 0.0 & & 0.0 & \\
\hline$N$ & & $\mathrm{~N}$ & & $\mathrm{~N}$ & \\
\hline 249 & & 68 & & 276 & \\
\hline 63.0 & & 73.0 & & 17.0 & \\
\hline 1 & & 3 & & 1 & \\
\hline B & & C & & $F$ & \\
\hline
\end{tabular}

Southbound

LT TH RT

154

29

61

2.6

61

1.3

0.0

1.4

32

120

79

6

5

127

83

3.4

11

0.2

1

3.6

12

106.0

404

0.0

$\mathrm{N}$

12

17.0

Overali Intersection Results

Output Flow (vph) 4008

Degree of Sat. ( 8 ) 196

Tot. Travel (veh-km) 2592

Tot.Travime (veh-h) 157

Unif. Delay (veh-h) 24.3

Rand. Delay (veh-h) 81.1

Total Delay (veh-h) 105.5

Avg. Delay (sec/v) 94

Unif. Stops (vph) 3023

Unif. Stops (8) 75

Rand. Stops (vph) 1203

Rand. Stops ( 8 ) 30

Total Stops (vph) 4227

Total Stops (8) 105

Time Full (8) 0

Fuel Consumpt. (lit) 607

Disutility Index 102

Level of Service F 
TRANSYT-7F Release 10.2 -- Node Output Summary (Detailed)

SIMULATION RESULTS

Node Number: 5

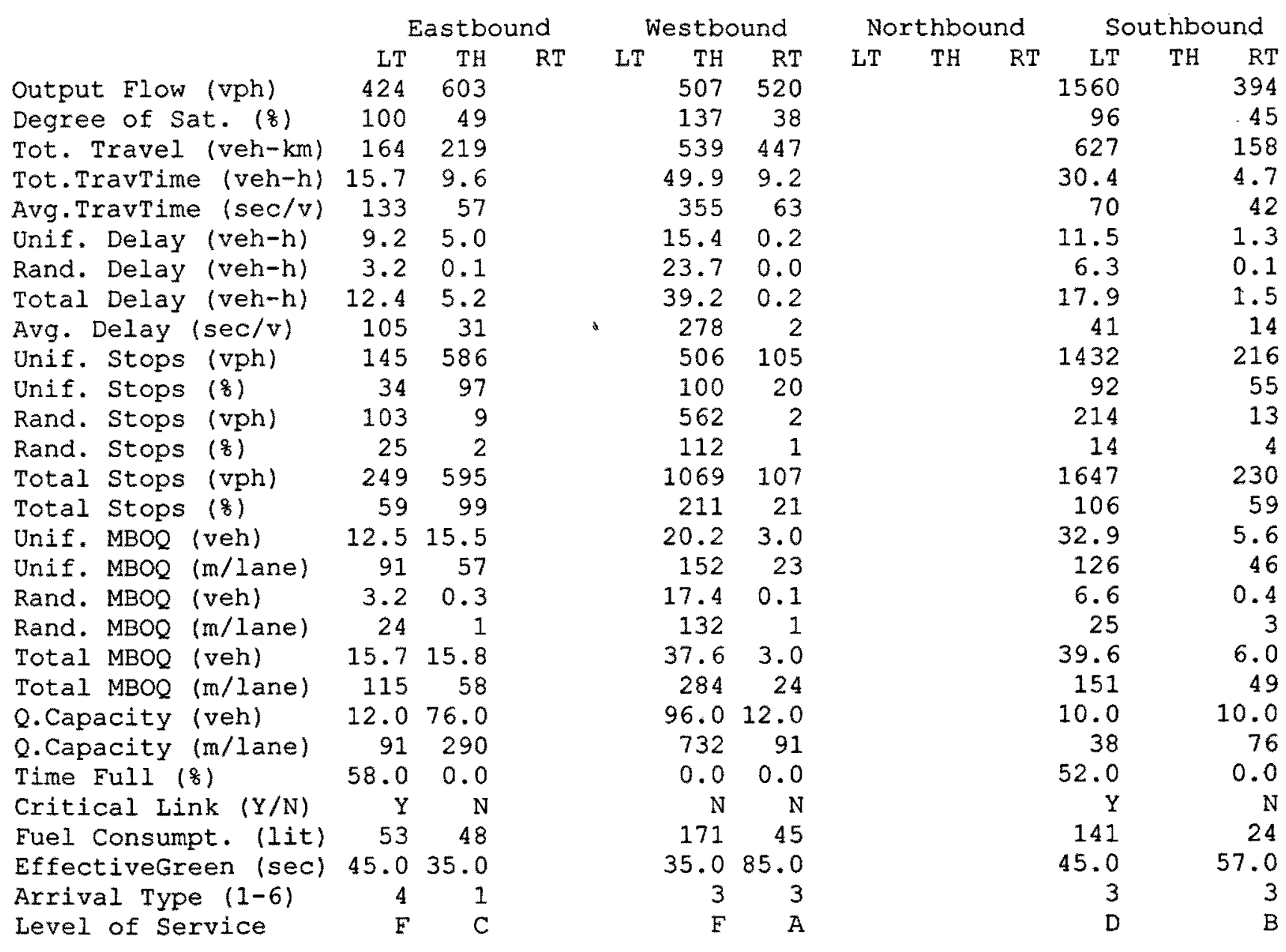

Overall Intersection Results

Output Flow (vph) 4008

Degree of Sat. (8) 137

Tot. Travel (veh-km) 2155

Tot.TravTime (veh-h) 119

Unif. Delay $(v e h-h) 42.9$

Rand. Delay (veh-h) 33.6

Total Delay (veh-h) 76.6

Avg. Delay $(\mathrm{sec} / \mathrm{v}) \quad 68$

Unif. Stops (vph) 2993

Unif. Stops (8) 75

Rand. Stops (vph) 906

Rand. Stops ( 8 ) 23

Total Stops (vph) 3899

Total Stops $(8) \quad 97$

Time Full (z) 110

Euel Consumpt. (1it) 484

$\begin{array}{lr}\text { Disutility Index } & 79 \\ \text { Level of Service } & E\end{array}$ 
TRANSYT-7F Release 10.2 -- Node Output Summary (Detailed)

SIMULATION RESULTS

Node Number: 6

\begin{tabular}{|c|c|c|c|}
\hline \multicolumn{2}{|c|}{ Northbound } & \multicolumn{2}{|c|}{ Southbound } \\
\hline LT & $\mathrm{RT}$ & LT & $\mathrm{RT}$ \\
\hline 770 & & 949 & 20 \\
\hline 52 & & 232 & 3 \\
\hline 329 & & 381 & 8 \\
\hline 10.1 & & 191.6 & 0.2 \\
\hline 47 & & 727 & 43 \\
\hline 3.2 & & 25.1 & 0.0 \\
\hline 0.2 & & 158.8 & 0.0 \\
\hline 3.5 & & 184.0 & 0.0 \\
\hline 16 & & 698 & 14 \\
\hline 548 & & 949 & 10 \\
\hline 71 & & 100 & 51 \\
\hline 16 & & 1000 & 0 \\
\hline 3 & & 106 & 3 \\
\hline 564 & & 1949 & 10 \\
\hline 74 & & 206 & 53 \\
\hline 14.2 & & 33.6 & 0.3 \\
\hline 53 & & 259 & 0 \\
\hline 0.5 & & 68.4 & 0.0 \\
\hline 2 & & 521 & 0 \\
\hline 14.7 & & 102.0 & 0.3 \\
\hline 55 & & 780 & 0 \\
\hline 114.0 & & 40.0 & 3.0 \\
\hline 434 & & 305 & 23 \\
\hline 0.0 & & 0.0 & 0.0 \\
\hline $\mathrm{N}$ & & $N$ & $\mathrm{~N}$ \\
\hline 53 & & 567 & 1 \\
\hline 47.0 & & 47.0 & 47.0 \\
\hline 1 & & 3 & 3 \\
\hline B & & $\mathrm{E}$ & B \\
\hline
\end{tabular}

Overall Intersection Results

$\begin{array}{lr}\text { Output Flow (vph) } & 3047 \\ \text { Degree of Sat. (q) } & 232 \\ \text { Tot. Travel (veh-km) } & 1274 \\ \text { Tot. TravTime (veh-h) } & 320 \\ \text { Unif. Delay (veh-h) } & 58.7 \\ \text { Rand. Delay (veh-h) } & 236.3 \\ \text { Total Delay (veh-h) } & 295.0 \\ \text { Avg. Delay (sec/v) } & 348 \\ \text { Unif. Stops (vph) } & 2779 \\ \text { Unif. Stops (8) } & 91 \\ \text { Rand. Stops (vph) } & 2097 \\ \text { Rand. Stops (8) } & 69 \\ \text { Total Stops (vph) } & 4877 \\ \text { Total Stops (8) } & 160 \\ \text { Time Eull (8) } & 17 \\ \text { Fuel Consumpt. (lit) } & 1001 \\ \text { Disutility Index } & 245 \\ \text { Level of Service } & \text { F }\end{array}$

\begin{tabular}{rr}
\multicolumn{3}{c}{ Westbound } \\
LT TH & RT \\
626 & 48 \\
194 & 7 \\
254 & 46 \\
104.9 & 1.5 \\
603 & 113 \\
24.7 & 0.5 \\
75.1 & 0.0 \\
99.8 & 0.5 \\
574 & 44 \\
626 & 108 \\
100 & 226 \\
1000 & 0 \\
160 & 1 \\
1626 & 108 \\
260 & 227 \\
28.1 & 3.0 \\
213 & 23 \\
38.7 & 0.0 \\
295 & 0 \\
66.8 & 3.0 \\
508 & 23 \\
26.0 & 3.0 \\
198 & 23 \\
11.0 & 6.0 \\
$Y$ & $\mathrm{~N}$ \\
315 & 8 \\
43.0 & 43.0 \\
1 & 1 \\
$\mathrm{~F}$ & $\mathrm{D}$
\end{tabular}

\begin{tabular}{lrr} 
& \multicolumn{3}{c}{ Eastbound } \\
& LT & RT \\
Output Flow (vph) & 590 & 44 \\
Degree of Sat. (q) & 85 & 8 \\
Tot. Travel (veh-km) & 237 & 17 \\
Tot.TravTime (veh-h) & 11.4 & 0.6 \\
Avg.TravTime (sec/v) & 69 & 52 \\
Unif. Delay (veh-h) & 4.5 & 0.2 \\
Rand. Delay (veh-h) & 2.1 & 0.0 \\
Total Delay (veh-h) & 6.6 & 0.2 \\
Avg. Delay (sec/v) & 40 & 23 \\
Unif. Stops (vph) & 507 & 28 \\
Unif. Stops (g) & 86 & 66 \\
Rand. Stops (vph) & 79 & 1 \\
Rand. Stops (8) & 14 & 4 \\
Total Stops (vph) & 587 & 30 \\
Total Stops (q) & 100 & 69 \\
Unif. MBOQ (veh) & 12.6 & 0.8 \\
Unif. MBOQ (m/lane) & 99 & 8 \\
Rand. MBOQ (veh) & 2.5 & 0.0 \\
Rand. MBOQ (m/lane) & 19 & 0 \\
Total MBOQ (veh) & 15.0 & 0.9 \\
Total MBOQ (m/lane) & 118 & 8 \\
Q.Capacity (veh) & 49.0 & 3.0 \\
Q.Capacity (m/lane) & 373 & 23 \\
Time Full (q) & 0.0 & 0.0 \\
Critical Link (Y/N) & $\mathrm{N}$ & $\mathrm{N}$ \\
Fuel Consumpt. (lit) & 52 & 3 \\
EffectiveGreen (sec) & 33.0 & 33.0 \\
Arrival Type (1-6) & 3 & 3 \\
Level of Service & $\mathrm{D}$ & $\mathrm{C}$ \\
& &
\end{tabular}

\begin{tabular}{|c|c|c|}
\hline & \multicolumn{2}{|c|}{ Eastbound } \\
\hline & $\mathrm{LT}$ & RT \\
\hline Output Flow (vph) & 590 & 44 \\
\hline Degree of Sat. (z) & 85 & 8 \\
\hline Tot. Travel (veh-km) & 237 & 17 \\
\hline Tot.TravTime (veh-h) & 11.4 & 0.6 \\
\hline Avg.TravTime (sec/v) & 69 & 52 \\
\hline Unif. Delay (veh-h) & 4.5 & 0.2 \\
\hline Rand. Delay (veh-h) & 2.1 & 0.0 \\
\hline Total Delay (veh-h) & 6.6 & 0.2 \\
\hline Avg. Delay (sec/v) & 40 & 23 \\
\hline Unif. Stops (vph) & 507 & 28 \\
\hline Unif. Stops $(8)$ & 86 & 66 \\
\hline Rand. Stops (vph) & 79 & 1 \\
\hline Rand, Stops (8) & 14 & 4 \\
\hline Total Stops (vph) & 587 & 30 \\
\hline Total Stops $(8)$ & 100 & 69 \\
\hline Unif. MBOQ (veh) & 12.6 & 0.8 \\
\hline Unif. MBOQ (m/lane) & 99 & 8 \\
\hline Rand. MBOQ (veh) & 2.5 & 0.0 \\
\hline Rand. MBOQ (m/lane) & 19 & 0 \\
\hline Total MBOQ (veh) & 15.0 & 0.9 \\
\hline Total MBOQ (m/lane) & 118 & 8 \\
\hline Q.Capacity (veh) & 49.0 & 3.0 \\
\hline Q. Capacity (m/lane) & 373 & 23 \\
\hline Time Full (8) & 0.0 & 0.0 \\
\hline Critical Link $(Y / N)$ & $\mathrm{N}$ & $\mathrm{N}$ \\
\hline Euel Consumpt. (1it) & 52 & 3 \\
\hline EffectiveGreen (sec) & 33.0 & 33.0 \\
\hline Arrival Type $(1-6)$ & 3 & 3 \\
\hline Level of Service & $\mathrm{D}$ & c \\
\hline
\end{tabular}

\author{
政
}


TRANSYT-7F Release 10.2 - Node Output Summary (Detailed) SIMULATION RESULTS

Node Number: 7

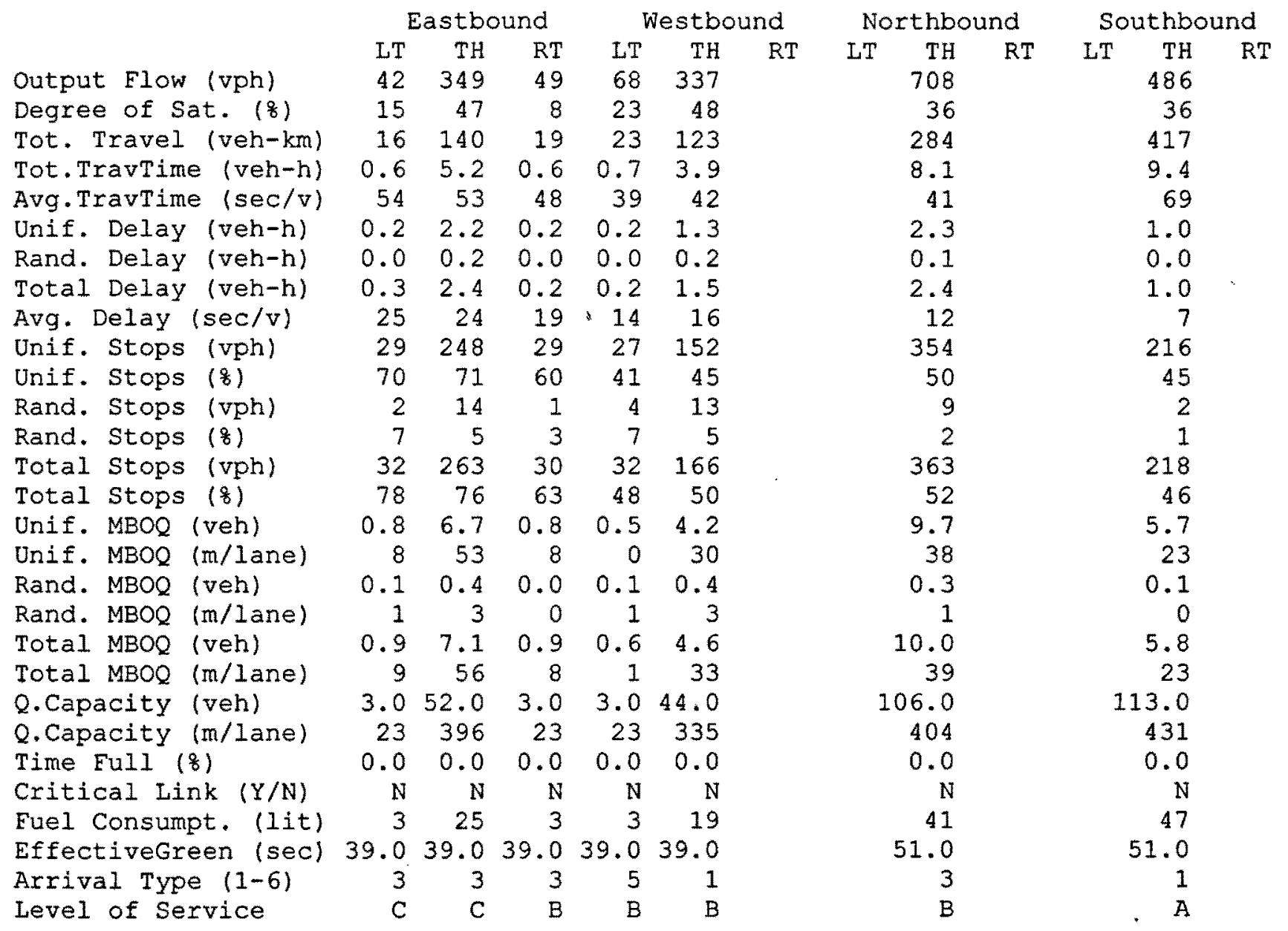

Overall Intersection Results

Output Flow (vph) 2039

Degree of Sat. ( 8 ) 48

Tot. Travel (veh-km) 1025

Tot.TravTime (veh-h) 28

Unif. Delay (veh-h) 7.6

Rand. Delay (veh-h) 0.5

Total Delay $($ veh-h) 8.2

Avg. Delay $(\mathrm{sec} / \mathrm{v}) \quad 14$

Unif. Stops (vph) 1059

Unif. Stops (8) 52

Rand. Stops (vph) 47

Rand. Stops (8) 2

Total Stops (vph) 1106

Total stops (8) 54

Time Full (z) 0

Euel Consumpt. (1it) 144

Disutility Index 12

Level of Service $B$ 
TRANSYT-7F Release 10.2 -- Node Output Summary (Detailed)

SIMULATION RESULTS

Node Number: 8

\begin{tabular}{|c|c|c|c|c|c|c|c|c|c|c|c|c|}
\hline & \multicolumn{3}{|c|}{ Eastbound } & \multicolumn{3}{|c|}{ Westbound } & \multicolumn{3}{|c|}{ Northbound } & \multicolumn{3}{|c|}{ Southbound } \\
\hline & LT & TH & $\mathrm{RT}$ & LT & $\mathrm{TH}$ & RT & LT & $\mathrm{TH}$ & $\mathrm{RT}$ & $\mathrm{LT}$ & $\mathrm{TH}$ & 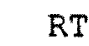 \\
\hline Output Flow (vph) & 33 & 489 & & 46 & 307 & & 99 & 133 & & & 396 & \\
\hline Degree of Sat. (8) & 7 & 50 & & 14 & 31 & & 41 & 21 & & & 58 & \\
\hline Tot. Travel (veh-km) & 11 & 189 & & 18 & 123 & & 41 & 55 & & & 159 & \\
\hline Tot.TravTime (veh-h) & 0.3 & 5.9 & & 0.6 & 3.7 & & 1.8 & 2.1 & & & 5.8 & \\
\hline Avg.TravTime (sec/v) & 35 & 43 & & 47 & 43 & & 66 & 58 & & & 53 & \\
\hline Unif. Delay (veh-h) & 0.1 & 1.9 & & 0.2 & 1.1 & & 0.9 & 1.0 & & & 2.2 & \\
\hline Rand. Delay (veh-h) & 0.0 & 0.2 & & 0.0 & 0.0 & & 0.1 & 0.0 & & & 0.4 & \\
\hline Total Delay (veh-h) & 0.1 & 2.1 & & 0.2 & 1.2 & & 1.0 & 1.0 & & & 2.6 & \\
\hline Avg. Delay (sec/v) & 11 & 15 & & 19 & 14 & & 36 & 28 & & & 24 & \\
\hline Unif. Stops (vph) & 10 & 254 & & 27 & 165 & & 55 & 87 & & & 274 & \\
\hline Unif. Stops (8) & 32 & 52 & & 59 & 54 & & 56 & 66 & & & 69 & \\
\hline Rand. Stops (vph) & 1 & 14 & & 2 & 7 & & 7 & 3 & & & 22 & \\
\hline Rand. Stops (8) & 4 & 3 & & 6 & 3 & & 8 & 3 & & & 6 & \\
\hline Total Stops (vph) & 11 & 268 & & 29 & 173 & & 63 & 90 & & & 296 & \\
\hline Total Stops (8) & 35 & 55 & & 65 & 57 & & 64 & 69 & & & 75 & \\
\hline Unif. MBOQ (veh) & 0.3 & 6.9 & & 0.8 & 4.5 & & 1.5 & 2.4 & & & 7.1 & \\
\hline Unif. $\mathrm{MBOQ}$ (m/lane) & 0 & 53 & & 8 & 38 & & 15 & 15 & & & 53 & \\
\hline Rand. MBOQ (veh) & 0.0 & 0.4 & & 0.1 & 0.2 & & 0.2 & 0.1 & & & 0.7 & \\
\hline Rand. MBOQ (m/lane) & 0 & 3 & & 1 & 2 & & 2 & 1 & & & 5 & \\
\hline Total MBOQ (veh) & 0.3 & 7.3 & & 0.8 & 4.7 & & 1.8 & 2.5 & & & 7.8 & \\
\hline Total MBOQ (m/lane) & 0 & 56 & & 9 & 40 & & 17 & 16 & & & 58 & \\
\hline Q.Capacity (veh) & 3.0 & 44.0 & & 3.0 & 53.0 & & 3.0 & 54.0 & & & 52.0 & \\
\hline Q.Capacity (m/lane) & 23 & 335 & & 23 & 404 & & 23 & 411 & & & 396 & \\
\hline Time Full (8) & 0.0 & 0.0 & & 0.0 & 0.0 & & 0.0 & 0.0 & & & 0.0 & \\
\hline Critical Link $(\mathrm{Y} / \mathrm{N})$ & $\mathbf{N}$ & $\mathbb{N}$ & & $\mathrm{N}$ & $\mathrm{N}$ & & $\mathrm{N}$ & $\mathrm{N}$ & & & $\mathrm{N}$ & \\
\hline Fuel Consumpt. (lit) & 1 & 29 & & 3 & 18 & & 7 & 10 & & & 28 & \\
\hline EffectiveGreen (sec) & 52.0 & 52.0 & & 52.0 & 52.0 & & 38.0 & 38.0 & & & 38.0 & \\
\hline Arrival Type $(1-6)$ & 4 & 1 & & 3 & 3 & & 3 & 1 & & & 3 & \\
\hline Level of Service & B & B & & B & B & & $\mathrm{D}$ & $\mathrm{C}$ & & & C & \\
\hline
\end{tabular}

Overall Intersection Results

$\begin{array}{lr}\text { Output Flow (vph) } & 1503 \\ \text { Degree of Sat. ( } 8) & 58 \\ \text { Tot. Travel (veh-km) } & 598 \\ \text { Tot.TravTime (veh-h) } & 20 \\ \text { Unif. Delay (veh-h) } & 7.6 \\ \text { Rand. Delay (veh-h) } & 0.8 \\ \text { Total Delay (veh-h) } & 8.5 \\ \text { Avg. Delay (sec/v) } & 20 \\ \text { Unif. Stops (vph) } & 874 \\ \text { Unif. Stops (8) } & 58 \\ \text { Rand. Stops (vph) } & 58 \\ \text { Rand. Stops (8) } & 4 \\ \text { Total Stops (vph) } & 933 \\ \text { Total Stops (8) } & 62 \\ \text { Time Eull (8) } & 0 \\ \text { Fuel Consumpt. (lit) } & 100 \\ \text { Disutility Index } & 11 \\ \text { Level of Service } & \mathrm{C}\end{array}$


Node Number: 9

Output Flow (vph)

Degree of Sat. (z)

Tot. Travel (veh-km)

Tot.TravTime (veh-h)

Avg.TravTime ( $\mathrm{sec} / \mathrm{v})$

Unif. Delay (veh-h)

Rand. Delay (veh-h)

Total Delay (veh-h)

Avg. Delay (sec/v)

Unif. Stops (vph)

Unif. Stops ( 8 )

Rand. Stops (vph)

Rand. Stops ( $(z)$

Total Stops (vph)

Total Stops (z)

Unif. MBOQ (veh)

Unif. MBOQ (m/lane)

Rand. MBOQ (veh)

Rand. $M B O Q$ (m/lane)

Total MBOQ (veh)

Total MBOQ (m/lane)

Q.Capacity (veh)

Q.Capacity (m/lane)

Time Full (o)

Critical Link $(\mathrm{Y} / \mathrm{N})$

Fuel Consumpt. (lit)

EffectiveGreen (sec)

Arrival Type $(1-6)$

Level of Service

\begin{tabular}{|c|c|c|c|c|c|c|c|c|c|c|}
\hline \multicolumn{2}{|c|}{ Eastbound } & \multicolumn{3}{|c|}{ Westbound } & \multicolumn{3}{|c|}{ Northbound } & \multicolumn{3}{|c|}{ Southbound } \\
\hline $\mathrm{TH}$ & RT & LT & $\mathrm{TH}$ & RT & $\mathrm{LT}$ & $\mathrm{TH}$ & RT & LT & TH & RT \\
\hline 753 & & & 316 & & & 374 & & & 391 & \\
\hline 69 & & & 31 & & & 68 & & & 159 & \\
\hline 302 & & & 250 & & & 150 & & & 161 & \\
\hline 9.0 & & & 5.7 & & & 6.3 & & & 39.2 & \\
\hline 43 & & & 65 & & & 61 & & & 361 & \\
\hline 2.3 & & & 0.7 & & & 2.7 & & & 5.0 & \\
\hline 0.7 & & & 0.0 & & & 0.6 & & & 30.9 & \\
\hline 3.0 & & & 0.7 & & & 3.3 & & & 35.9 & " \\
\hline 14 & s & & 8 & & & 32 & & & 331 & \\
\hline 424 & & & 114 & & & 290 & & & 365 & \\
\hline 56 & & & 36 & & & 78 & & & 93 & \\
\hline 34 & & & 6 & & & 32 & & & 625 & \\
\hline 5 & & & 3 & & & 9 & & & 161 & \\
\hline 459 & & & 121 & & & 322 & & & 991 & \\
\hline 61 & & & 39 & & & 87 & & & 254 & \\
\hline 10.0 & & & 3.3 & & & 7.5 & & & 7.7 & \\
\hline 76 & & & 23 & & & 61 & & & 61 & \\
\hline 1.1 & & & 0.2 & & & 1.0 & & & 19.3 & \\
\hline 8 & & & 2 & & & 8 & & & 147 & \\
\hline 11.0 & & & 3.5 & & & 8.5 & & & 27.1 & \\
\hline 84 & & & 25 & & & 69 & & & 208 & \\
\hline 52.0 & & & 98.0 & & & 53.0 & & & 52.0 & \\
\hline 396 & & & 747 & & & 404 & & & 396 & \\
\hline 0.0 & & & 0.0 & & & 0.0 & & & 0.0 & \\
\hline $\mathrm{N}$ & & & $\mathrm{N}$ & & & $\mathrm{N}$ & & & $\mathrm{N}$ & \\
\hline 46 & & & 28 & & & 30 & & & 123 & \\
\hline 59.0 & & & 59.0 & & & 31.0 & & & 31.0 & \\
\hline 3 & & & 1 & & & 3 & & & 1 & \\
\hline B & & & A & & & c & & & $F$ & \\
\hline
\end{tabular}

Overall Intersection Results

Output Flow (vph) 1834

Degree of Sat. (8) 159

Tot. Travel (veh-km) 865

Tot. TravTime (veh-h) 60

Unif. Delay (veh-h) 10.7

Rand. Delay (veh-h) 32.3

Total Delay (veh-h) 43.1

Avg. Delay (sec/v) 84

Unif. Stops (vph) 1194

Unif. Stops $(8) \quad 65$

Rand. Stops (vph) 699

Rand. Stops (8) 38

Total Stops (vph) 1894

Total Stops (8) 103

Time Full (8) 0

Fuel Consumpt. (lit) 228

Disutility Index 42

Level of Service 
Optimization for Splits Results 
Node Number: 1

Output Flow (vph)

Degree of Sat. ( $(8)$

Tot. Travel (veh-km)

Tot.TravTime (veh-h)

Avg.TravTime (sec/v)

Unif. Delay (veh-h)

Rand. Delay (veh-h)

Total Delay (veh-h)

Avg. Delay $(\mathrm{sec} / \mathrm{v})$

Unif. Stops (vph)

Unif. Stops ( $(8)$

Rand. Stops (vph)

Rand. Stops (8)

Total Stops (vph)

Total Stops $(z)$

Unif. MBOQ (veh)

Unif. MBOQ (m/lane)

Rand. MBOQ (veh)

Rand. MBOQ (m/lane)

Total MBOQ (veh)

Total MBOQ (m/lane)

Q. Capacity (veh)

Q.Capacity (m/lane)

Time Full (8)

Critical Link ( $Y / N)$

Fuel Consumpt. (lit)

EffectiveGreen (sec)

Arrival Type (1-6)

Level of Service

\begin{tabular}{|c|c|c|c|c|c|c|c|c|c|}
\hline \multicolumn{2}{|c|}{ Eastbound } & \multicolumn{3}{|c|}{ Westbound } & \multicolumn{2}{|c|}{ Northbound } & \multicolumn{3}{|c|}{ Southbound } \\
\hline LT & RT & LT & $\mathrm{TH}$ & $\mathrm{RT}$ & LT & RT & LT & $\mathrm{TH}$ & $\mathrm{RT}$ \\
\hline 848 & 107 & 32 & 274 & & 591 & & 74 & 442 & \\
\hline 106 & 12 & 38 & 27 & & 46 & & 33 & 68 & \\
\hline 674 & 80 & 12 & 110 & & 237 & & 41 & 238 & \\
\hline 27.8 & 2.1 & 0.5 & 3.1 & & 8.4 & & 1.8 & 10.4 & \\
\hline 118 & 71 & 59 & 41 & & 51 & & 89 & 84 & \\
\hline 4.1 & 0.5 & 0.1 & 0.9 & & 3.4 & & 0.9 & 4.9 & \\
\hline 10.1 & 0.0 & 0.1 & 0.0 & & 0.1 & & 0.0 & 0.6 & \\
\hline 14.3 & 0.5 & 0.2 & 0.9 & & 3.6 & & 1.0 & 5.6 & \\
\hline 61 & 17 & 30 & 12 & & 22 & & 49 & 45 & \\
\hline 609 & 70 & 26 & 135 & & 401 & & 61 & 383 & \\
\hline 72 & 66 & 83 & 50 & & 68 & & 84 & 87 & \\
\hline 309 & 1 & 9 & 6 & & 13 & & 7 & 31 & \\
\hline 37 & 2 & 30 & 3 & & 3 & & 10 & 8 & \\
\hline 919 & 72 & 35 & 141 & & 415 & & 69 & 414 & \\
\hline 109 & 68 & 113 & 52 & & 71 & & 94 & 94 & \\
\hline 13.4 & 2.0 & 0.4 & 3.7 & & 10.7 & & 1.7 & 10.5 & \\
\hline 99 & 15 & 0 & 30 & & 42 & & 15 & 84 & \\
\hline 9.6 & 0.0 & 0.3 & 0.2 & & 0.4 & & 0.2 & 1.0 & \\
\hline 73 & 0 & 2 & 1 & & 2 & & 2 & 7 & \\
\hline 23.0 & 2.0 & 0.7 & 3.9 & & 11.1 & & 1.9 & 11.5 & \\
\hline 172 & 15 & 2 & 31 & & 44 & & 17 & 91 & \\
\hline 93.0 & 3.0 & 3.0 & 53.0 & & 106.0 & & 3.0 & 50.0 & \\
\hline 709 & 23 & 23 & 404 & & 404 & & 23 & 381 & \\
\hline 0.0 & 0.0 & 0.0 & 0.0 & & 0.0 & & 0.0 & 0.0 & \\
\hline $\mathrm{N}$ & $\mathrm{N}$ & $\mathrm{N}$ & $\mathrm{N}$ & & $\mathbf{N}$ & & $\mathrm{N}$ & $\mathrm{N}$ & \\
\hline 117 & 10 & 2 & 16 & & 41 & & 8 & 46 & \\
\hline 55.0 & 55.0 & 55.0 & 55.0 & & 35.0 & & 35.0 & 35.0 & \\
\hline 1 & 2 & 3 & 3 & & 3 & & 1 & 1 & \\
\hline $\mathrm{E}$ & B & C & B & & C & & D & D & \\
\hline
\end{tabular}

Overall Intersection Results

Output Flow (vph) 2368

Degree of Sat. (8) 106

Tot. Travel (veh-km) 1395

Tot.TravTime (veh-h) 54

Unif. Delay (veh-h) 15.1

Rand. Delay (veh-h) 11.2

Total Delay (veh-h) 26.4

Avg. Delay (sec/v) 40

Unif. Stops (vph) 1689

Unif. Stops ( 8 ) 71

Rand. Stops (vph) 378

Rand. Stops (8) 16

Total Stops (vph) 2067

Total Stops (8) 87

Time Full ( 8$) \quad 0$

Fuel Consumpt. (lit) 243

Disutility Index 31

Level of Service 
Node Number: 2

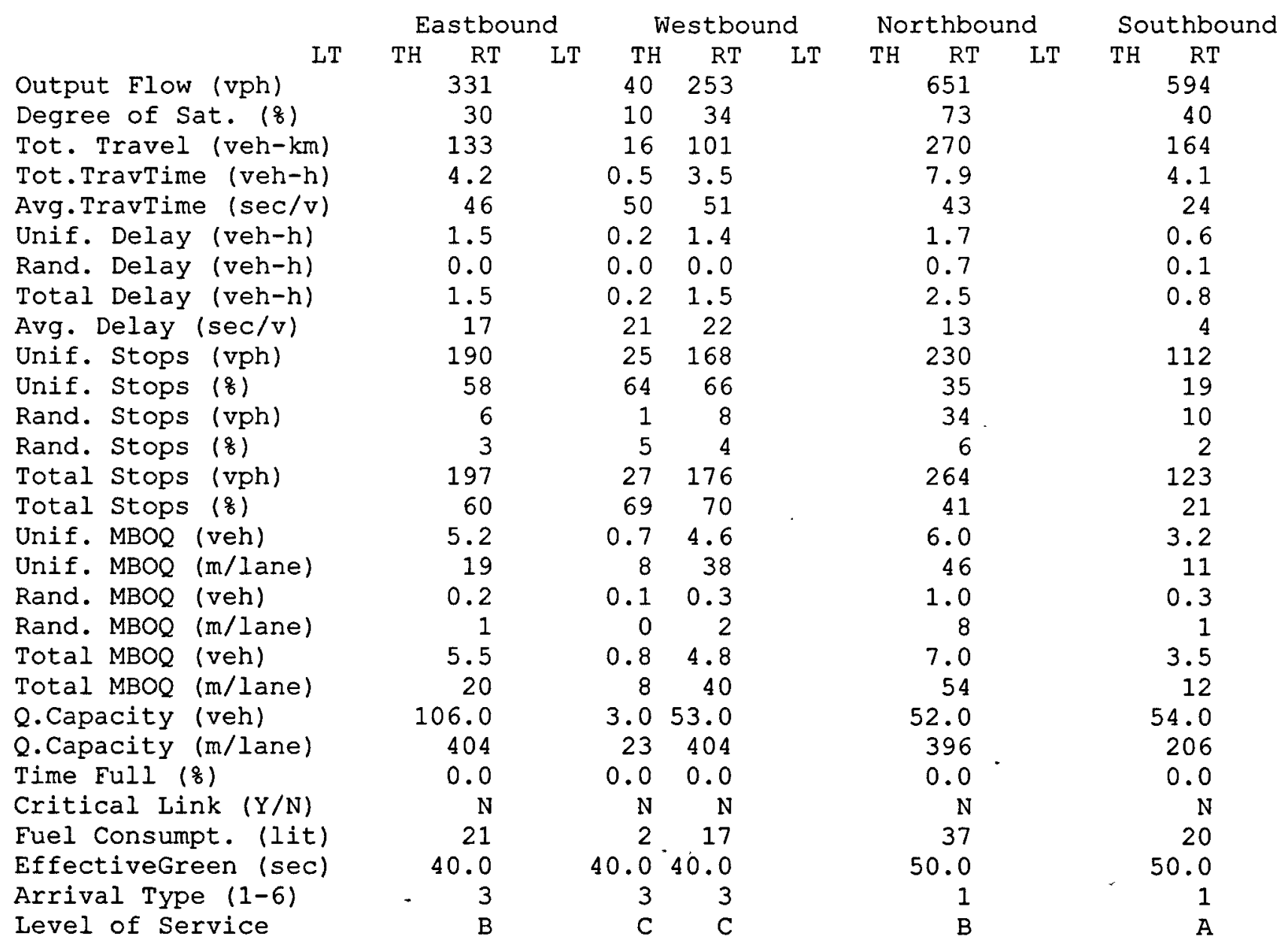

Overall Intersection Results

$\begin{array}{lr}\text { Output Flow (vph) } & 1869 \\ \text { Degree of Sat. ( } 8) & 73 \\ \text { Tot. Travel (veh-km) } & 685 \\ \text { Tot. TravTime (veh-h) } & 20 \\ \text { Unif. Delay (veh-h) } & 5.6 \\ \text { Rand. Delay (veh-h) } & 1.0 \\ \text { Total Delay (veh-h) } & 6.7 \\ \text { Avg. Delay (sec/v) } & 12 \\ \text { Unif. Stops (vph) } & 727 \\ \text { Unif. Stops (8) } & 39 \\ \text { Rand. Stops (vph) } & 61 \\ \text { Rand. Stops ( } 8) & 3 \\ \text { Total Stops (vph) } & 788 \\ \text { Total Stops ( } 8) & 42 \\ \text { Time Full (8) } & 0 \\ \text { Fuel Consumpt. (lit) } & 100 \\ \text { Disutility Index } & 9 \\ \text { Level of Service } & \text { B }\end{array}$


TRANSYT-7F Release 10.2 -- Node Output Summary (Detailed) OPTIMIZATION FOR SPLITS

Node Number: 3

Output Flow (vph)

Degree of Sat. ( $(8)$

Tot. Travel (veh-km)

Tot. TravTime (veh-h)

Avg. TravTime ( $\mathrm{sec} / \mathrm{v})$

Unif. Delay (veh-h)

Rand. Delay (veh-h)

Total Delay (veh-h)

Avg. Delay (sec/v)

Unif. Stops (vph)

Unif. Stops $(8)$

Rand. Stops (vph)

Rand. Stops ( $(8)$

Total Stops (vph)

Total Stops (q)

Unif. MBOQ (veh)

Unif. MBOQ ( $\mathrm{m} /$ lane)

Rand. $M B O Q$ (veh)

Rand. $M B O Q$ ( $m /$ lane)

Total MBOQ (veh)

Total MBOQ (m/lane)

Q.Capacity (veh)

Q. Capacity (m/lane)

Time Full (z)

Critical Link ( $Y / N)$

Fuel Consumpt. (lit)

EffectiveGreen (sec)

Arrival Type $(1-6)$

Level of Service

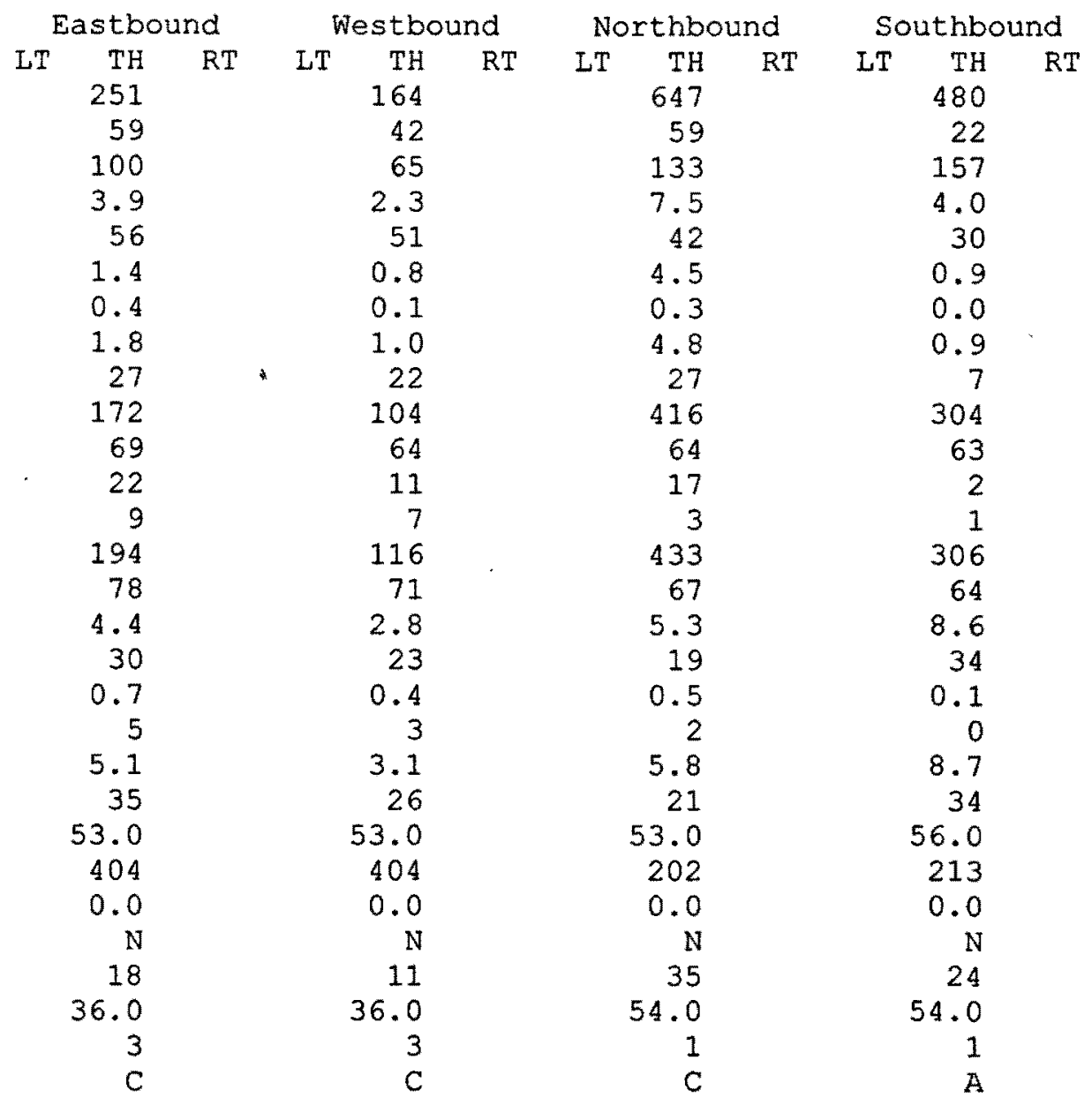

Overall Intersection Results

Output Flow (vph) 1542

Degree of Sat. ( 8$) \quad 59$

Tot. Travel (veh-km) 457

Tot.TravTime (veh-h) 17

Unif. Delay (veh-h) 7.8

Rand. Delay (veh-h) 0.8

Total Delay (veh-h) 8.7

Avg. Delay (sec/v) 20

Unif. Stops (vph) 997

Unif. Stops (8) 65

Rand. Stops (vph) 53

Rand. Stops (8) 3

Total Stops (vph) 1050

Total Stops (8) 68

Time Eull $(8) \quad 0$

Fuel Consumpt. (1it) 90

Disutility Index a 12

Level of Service $C$ 
TRANSYT-7F Release 10.2 -- Node Output Summary (Detailed)

OPTIMIZATION FOR SPLITS

Node Number: 4

Output Flow (vph)

Degree of Sat. ( $(8)$

Tot. Travel (veh-km)

Tot.TravTime (veh-h)

Avg.TravTime ( $\mathrm{sec} / \mathrm{v})$

Unif. Delay (veh-h)

Rand. Delay (veh-h)

Total Delay (veh-h)

Avg. Delay (sec/v)

Unif. Stops (vph)

Unif. Stops ( $(8)$

Rand. Stops (vph)

Rand. Stops ( 8 )

Total Stops (vph)

Total Stops (8)

Unif. MBOQ (veh)

Unif. MBOQ (m/lane)

Rand. MBOQ (veh)

Rand. MBOQ (m/lane)

Total MBOQ (veh)

Total MBOQ (m/lane)

Q.Capacity (veh)

Q. Capacity (m/lane)

Time Full (8)

Critical Link ( $Y / N)$

Fuel Consumpt. (lit)

EffectiveGreen (sec)

Arrival Type (1-6)

Level of Service

\begin{tabular}{|c|c|c|c|c|c|c|c|c|c|}
\hline \multicolumn{2}{|c|}{ Eastbound } & \multicolumn{2}{|c|}{ Westbound } & \multicolumn{3}{|c|}{ Northbound } & \multicolumn{3}{|c|}{ Southbound } \\
\hline TH & RT & LT & RT & LT & $\mathrm{TH}$ & RT & LT & $\mathrm{TH}$ & RT \\
\hline 2186 & & 1067 & & & 372 & & & 154 & \\
\hline 76 & & 84 & & & 213 & & & 49 & \\
\hline 1967 & & 428 & & & 134 & & & 61 & \\
\hline 42.3 & & 11.2 & & & 74.1 & & & 3.1 & \\
\hline 69 & & 37 & & & 717 & & & 73 & \\
\hline 2.6 & & 0.5 & & & 17.0 & & & 1.6 & \\
\hline 0.3 & & 2.0 & & & 54.3 & & & 0.2 & \\
\hline 3.0 & & 2.6 & & & 71.4 & & & 1.9 & \\
\hline 5 & & 8 & & & 691 & & & 44 & \\
\hline 1174 & & 237 & & & 371 & & & 135 & \\
\hline 54 & & 22 & & & 100 & & & 88 & \\
\hline 16 & & 79 & & & 823 & & & 15 & \\
\hline 1 & & 8 & & & 222 & & & 10 & \\
\hline 1191 & & 316 & & & 1195 & & & 150 & \\
\hline 55 & & 30 & & & 322 & & & 98 & \\
\hline 16.8 & & 4.4 & & & 17.3 & & & 3.8 & \\
\hline 65 & & 15 & & & 65 & & & 15 & \\
\hline 0.5 & & 2.4 & & & 25.4 & & & 0.5 & \\
\hline 2 & & 9 & & & 97 & & & 2 & \\
\hline 17.4 & & 6.8 & & & 42.7 & & & 4.2 & \\
\hline 67 & & 24 & & & 162 & & & 17 & \\
\hline 222.0 & & 105.0 & & & 56.0 & & & 16.0 & \\
\hline 846 & & 400 & & & 213 & & & 404 & \\
\hline 0.0 & & 0.0 & & & 0.0 & & & 0.0 & \\
\hline $\mathrm{N}$ & & $\mathrm{N}$ & & & $\mathrm{N}$ & & & $\mathrm{N}$ & \\
\hline 221 & & 53 & & & 219 & & & 14 & \\
\hline 72.0 & & 82.0 & & & 8.0 & & & 8.0 & \\
\hline 1 & & 3 & & & 1 & & 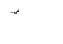 & 3 & \\
\hline A & & A & & & $F$ & & & D & \\
\hline
\end{tabular}

Overall Intersection Results

Output Flow (vph) 3779

Degree of Sat. (8) 213

Tot. Travel (veh-km) 2592

Tot.TravTime (veh-h) 130

Unif. Delay (veh-h) 21.9

Rand. Delay (veh-h) 57.0

Total Delay (veh-h) 78.9

Avg. Delay (sec/v) 75

Unif. Stops (vph) 1918

Unif. Stops $(8) \quad 51$

Rand. Stops (vph) 934

Rand. Stops $(z) \quad 25$

Total Stops (vph) 2853

Total stops ( 8 ) 76

Time Full (8) 0

Fuel Consumpt. (Iit) 508

Disutility Index 74

Level of Service $E$ 
TRANSYT-7F Release 10.2 -- Node Output Summary (Detailed) OPTIMIZATION FOR SPLITS

Node Number: 5

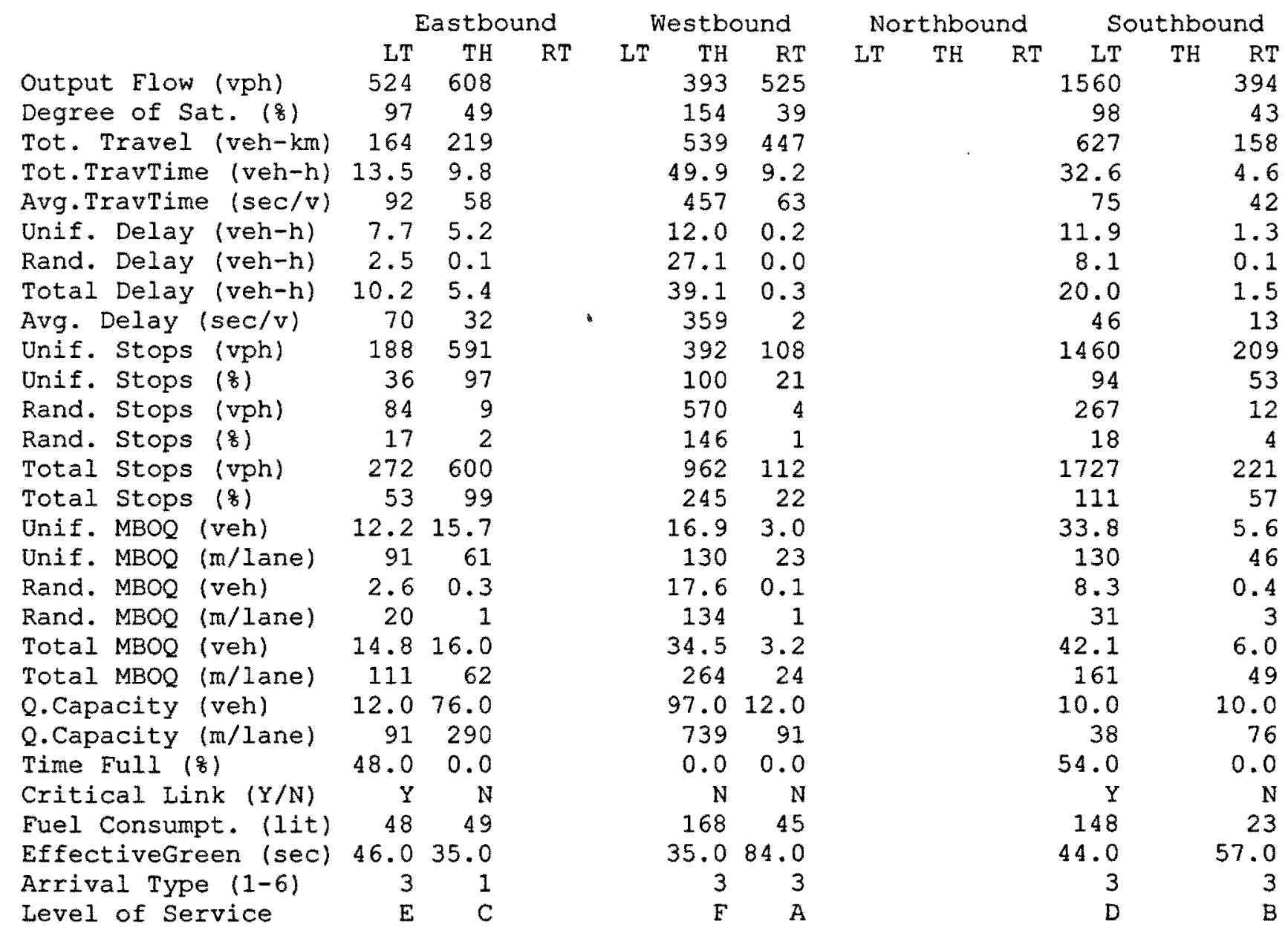

Overall Intersection Results

Output Flow (vph) 4004

Degree of Sat. (o 154

Tot. Travel (veh-km) 2155

Tot.TravTime (veh-h) 119

Unif. Delay (veh-h) 38.6

Rand. Delay (veh-h) 38.1

Total Delay (veh-h) 76.7

Avg. Delay ( $\mathrm{sec} / \mathrm{v}) \quad 69$

Unif. Stops (vph) 2950

Unif. Stops $(8) \quad 74$

Rand. Stops (vph) 947

Rand. Stops (8) 24

Total Stops (vph) 3898

Total stops (8) 97

Time Full (8) 102

Fuel Consumpt. (1it) 484

Disutility Index 79

Level of Service $E$ 
TRANSYT-7F Release 10.2 -- Node Output Summary (Detailed)

OPTIMIZATION FOR SPLITS

Node Number: 6

Output Flow (vph)

Degree of Sat. ( 8 )

Tot. Travel (veh-km)

Tot.TravTime (veh-h)

Avg.TravTime (sec/v)

Unif. Delay (veh-h)

Rand. Delay (veh-h)

Total Delay (veh-h)

Avg. Delay (sec/v)

Unif. Stops (vph)

Unif. Stops (o)

Rand. Stops (vph)

Rand. Stops ( 8 )

Total Stops (vph)

Total Stops ( 8$)$

Unif. MBOQ (veh)

Unif. $M B O Q$ ( $\mathrm{m} /$ lane)

Rand. MBOQ (veh)

Rand. $M B O Q$ ( $\mathrm{m} /$ lane)

Total MBOQ (veh)

Total MBOQ (m/lane)

Q.Capacity (veh)

Q.Capacity (m/lane)

Time Full (z)

Critical Link $(Y / N)$

Fuel Consumpt. (lit)

EffectiveGreen (sec)

Arrival Type (1-6)

Level of Service

\begin{tabular}{|c|c|c|c|}
\hline \multicolumn{2}{|c|}{ Eastbound } & \multicolumn{2}{|c|}{ Westbound } \\
\hline $\mathrm{LT}$ & RT & $\mathrm{LT}$ & $\mathrm{RT}$ \\
\hline 590 & 44 & 557 & 62 \\
\hline 88 & 9 & 166 & 9 \\
\hline 237 & 17 & 254 & 46 \\
\hline 11.9 & 0.6 & 76.7 & 1.7 \\
\hline 73 & 53 & 496 & 98 \\
\hline 4.7 & 0.2 & 23.9 & 0.7 \\
\hline 2.4 & 0.0 & 47.7 & 0.0 \\
\hline 7.2 & 0.2 & 71.6 & 0.7 \\
\hline 44 & 24 & 463 & 45 \\
\hline 516 & $29^{\circ}$ & 556 & 109 \\
\hline 87 & 67 & 100 & 177 \\
\hline 91 & 1 & 928 & 0 \\
\hline 16 & 4 & 167 & 1 \\
\hline 607 & 30 & 1484 & 109 \\
\hline 103 & 71 & 267 & 177 \\
\hline 12.9 & 0.8 & 26.2 & 3.0 \\
\hline 99 & 8 & 198 & 23 \\
\hline 2.8 & 0.0 & 28.6 & 0.0 \\
\hline 22 & 0 & 218 & 0 \\
\hline 15.7 & 0.9 & 54.8 & 3.0 \\
\hline 121 & 8 & 416 & 23 \\
\hline 49.0 & 3.0 & 26.0 & 3.0 \\
\hline 373 & 23 & 198 & 23 \\
\hline 0.0 & 0.0 & 19.0 & 6.0 \\
\hline $\mathrm{N}$ & $\mathrm{N}$ & $Y$ & $\mathrm{~N}$ \\
\hline 54 & 3 & 235 & 9 \\
\hline 32.0 & 32.0 & 42.0 & 42.0 \\
\hline 3 & 3 & 1 & 1 \\
\hline D & C & F & \\
\hline
\end{tabular}

Northbound

Southbound

LT TH RT LT TH RT

762

94920

50

$227 \quad 3$

329

$381 \quad 8$

9.9

46

$185.0 \quad 0.2$

$701 \quad 42$

$24.2 \quad 0.0$

$\begin{array}{ll}153.1 & 0.0\end{array}$

0.2

3.3

15

545

72

15

2

561

74

14.1

53

0.5

2

14.5

55

114.0

434

$0.0^{\circ}$

N

52

48.0

177.3

0.0

$672 \quad 13$

$949 \quad 9$

$100 \quad 50$

$1000 \quad 0$

1063

$1949 \quad 10$

$206 \quad 52$

$\begin{array}{ll}31.6 & 0.3\end{array}$

2440

$\begin{array}{ll}67.3 & 0.0\end{array}$

$513 \quad 0$

$\begin{array}{lll}98.9 & 0.3\end{array}$

$757 \quad 0$

$40.0 \quad 3.0$

30523

$0.0 \quad 0.0$

549

$48.0 \quad 48.0$

Overall Intersection Results

Output Flow (vph) 2984

Degree of Sat. (8) 227

Tot. Travel (veh-km) 1274

Tot.TravTime (veh-h) 286

Unif. Delay (veh-h) 57.1

Rand. Delay (veh-h) 203.6

Total Delay (veh-h) 260.7

Avg. Delay (sec/v) 314

Unif. Stops (vph) 2716

Unif. Stops $(8) \quad 91$

Rand. Stops (vph) 2036

Rand. Stops (8) 68

Total Stops (vph) 4752

Total Stops (8) 159

Time Full $(8) \quad 25$

Fuel Consumpt. (lit) 905

Disutility Index 219

Level of Service F 
TRANSYT-7F Release 10.2 -- Node Output Summary (Detailed) OPTIMIZATION FOR SPLITS

Node Number: 7

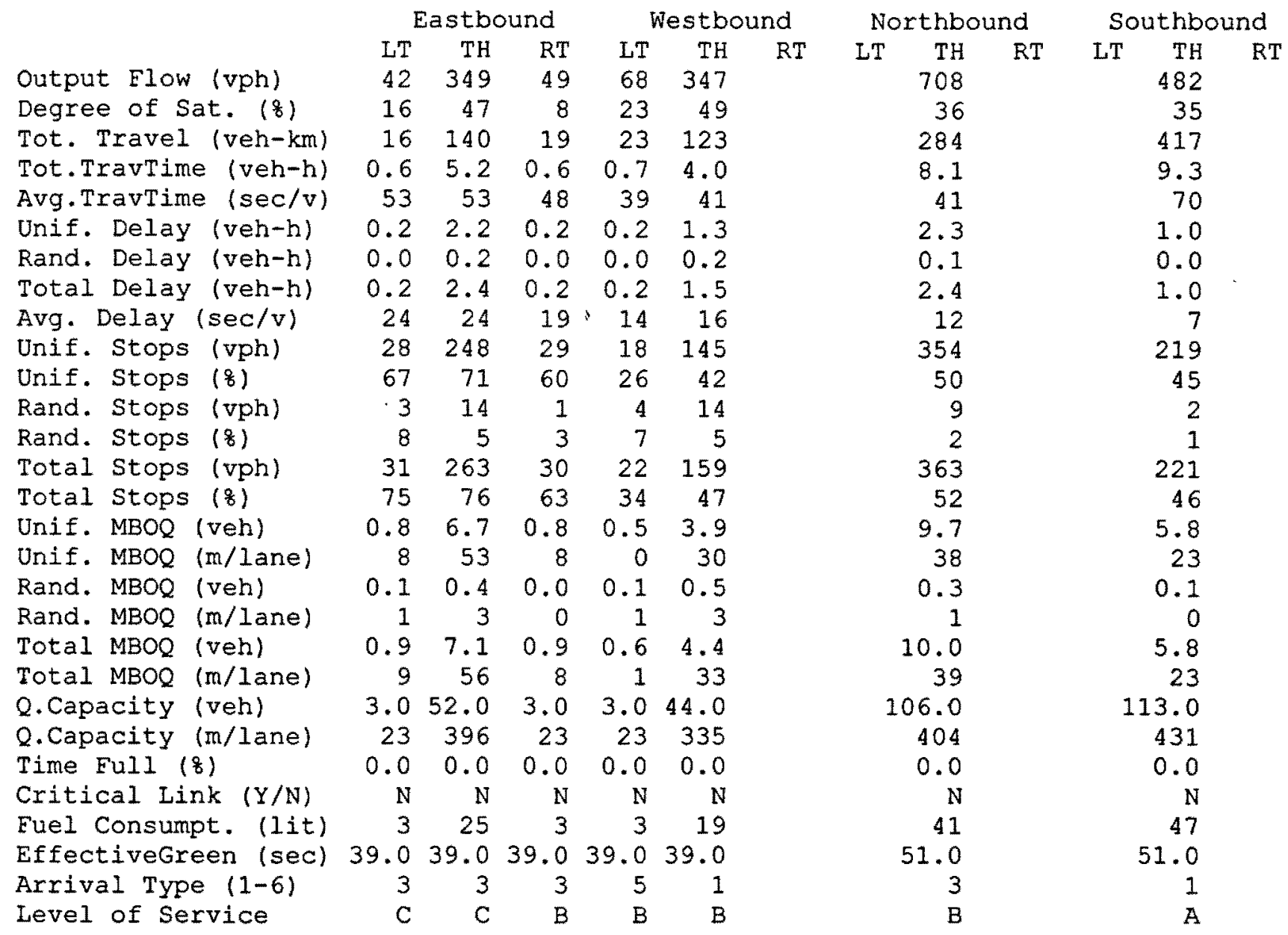

Overall Intersection Results

Output Flow (vph) 2045

Degree of Sat. (8) 49

Tot. Travel (veh-km) 1025

Tot.TravTime (veh-h) 28

Unif. Delay (veh-h) 7.6

Rand. Delay (veh-h) 0.6

Total Delay (veh-h) 8.2

Avg. Delay (sec/v) 14

Unif. Stops (vph) 1042

Unif. Stops $\left(\frac{8}{8}\right) \quad 51$

Rand. Stops (vph) 49

Rand. Stops $(8) \quad 2$

Total Stops (vph) 1091

Total stops $(8) \quad 53$

Time Full (8) 0

Fuel Consumpt. (1it) 143

Disutility Index 12

Level of Service B 
TRANSYT-7F Release 10.2 -- Node Output Summary (Detailed) OPTIMIZATION FOR SPLITS

Node Number: 8

Overall Intersection Results

$\begin{array}{lr}\text { Output Flow (vph) } & 1504 \\ \text { Degree of Sat. (8) } & 58 \\ \text { Tot. Travel (veh-km) } & 598 \\ \text { Tot. TravTime (veh-h) } & 20 \\ \text { Unif. Delay (veh-h) } & 7.3 \\ \text { Rand. Delay (veh-h) } & 0.7 \\ \text { Total Delay (veh-h) } & 8.1 \\ \text { Avg. Delay (sec/v) } & 19 \\ \text { Unif. Stops (vph) } & 842 \\ \text { Unif. Stops ( } 8 \text { ) } & 56 \\ \text { Rand. Stops (vph) } & 57 \\ \text { Rand. Stops ( } 8) & 4 \\ \text { Total Stops (vph) } & 899 \\ \text { Total Stops (8) } & 60 \\ \text { Time Full (8) } & 0 \\ \text { Fuel Consumpt. (lit) } & 98 \\ \text { Disutility Index } & 11 \\ \text { Level of Service } & \text { B }\end{array}$


TRANSYT-7F Release 10.2 -- Node Output Summary (Detailed)

OPTIMIZATION FOR SPLITS

Node Number: 9

Output Flow (vph)

Degree of Sat. (8)

Tot. Travel (veh-km)

Tot.TravTime (veh-h)

Avg.TravTime (sec/v)

Unif. Delay (veh-h)

Rand. Delay (veh-h)

Total Delay (veh-h)

Avg. Delay (sec/v)

Unif. Stops (vph)

Unif. Stops $(z)$

Rand. Stops (vph)

Rand. Stops ( 8 )

Total Stops (vph)

Total Stops (8)

Unif. MBOQ (veh)

Unif. MBOQ (m/lane)

Rand. $M B O Q$ (veh)

Rand. MBOQ ( $\mathrm{m} /$ lane)

Total MBOQ (veh)

Total MBOQ (m/lane)

Q.Capacity (veh)

Q.Capacity (m/lane)

Time Full (z)

Critical Link ( $Y / N)$

Fuel Consumpt. (Iit)

EffectiveGreen (sec)

Arrival Type $(1-6)$

Level of Service

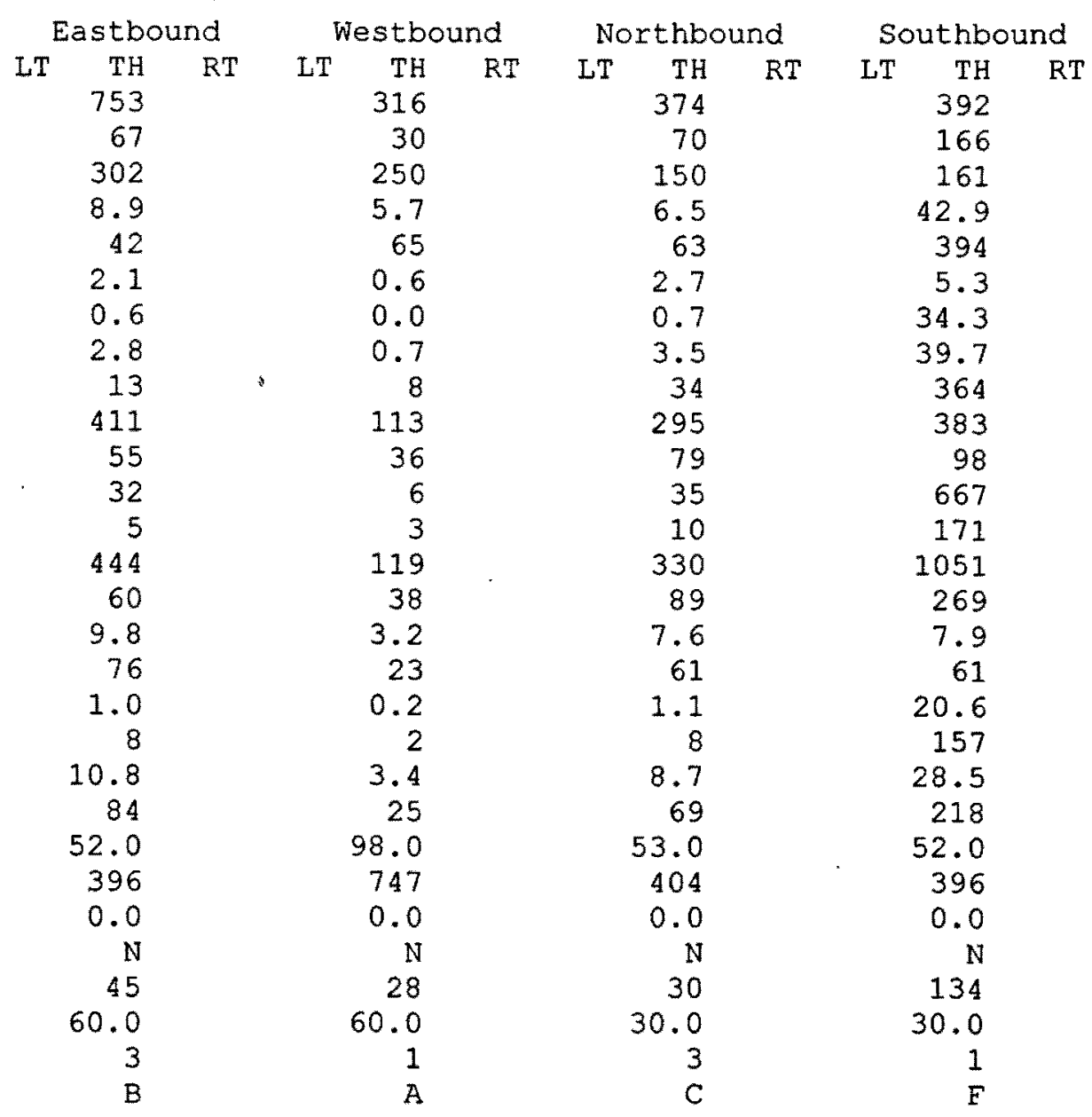

Overall Intersection Results

Output Flow (vph) 1835

Degree of Sat. (8) 166

Tot. Travel (veh-km) 865

Tot.TravTime (veh-h) 64

Unif. Delay (veh-h) 10.9

Rand. Delay (veh-h) 35.9

Total Delay (veh-h) 46.8

Avg. Delay (sec/v) 92

Unif. Stops (vph) 1203

Unif. Stops ( 8 ) 66

Rand. Stops (vph) 742

Rand. Stops (8) 40

Total Stops (vph) 1945

Total Stops $(8) \quad 106$

Time Full (8) 0

Fuel Consumpt. (lit) 239

Disutility Index 45

Level of Service F 


\section{Optimization for Cycle Length Results}


TRANSYT-7F Release 10.2 -- Node Output Summary (Detailed) OPTIMIZATION FOR CYCLE IENGTH

Node Number: 1

Output Flow (vph)

Degree of Sat. (z)

Tot. Travel (veh-km)

Tot.TravTime (veh-h)

Avg.TravTime (sec/v)

Unif. Delay (veh-h)

Rand. Delay (veh-h)

Total Delay (veh-h)

Avg. Delay (sec/v)

Unif. Stops (vph)

Unif. Stops $(8)$

Rand. Stops (vph)

Rand. Stops ( 8$)$

Total Stops (vph)

Total Stops (z)

Unif. MBOQ (veh)

Unif. MBOQ (m/lane)

Rand. $M B O Q$ (veh)

Rand. MBOQ (m/lane)

Total MBOQ (veh)

Total MBOQ (m/lane)

Q. Capacity (veh)

Q. Capacity (m/lane)

Time Full (8)

Critical Link ( $Y / N$ )

Fuel Consumpt. (lit)

EffectiveGreen (sec)

Arrival Type $(1-6)$

Level of Service
Eastbound

LT

$\begin{array}{rrrrr}\text { TH } & \text { RT } & \text { LT } & \text { TH } & \text { RT } \\ 850 & 107 & 32 & 274 & \\ 107 & 12 & 52 & 27 \\ 674 & 80 & 12 & 110 \\ 28.0 & 2.1 & 0.7 & 3.3 \\ 118 & 72 & 85 & 44 \\ 4.1 & 0.5 & 0.2 & 1.1 \\ 10.3 & 0.0 & 0.2 & 0.0 \\ 14.5 & 0.5 & 0.5 & 1.1 \\ 61 & 18 & 56 & 15 \\ 540 & 56 & 46 & 133 \\ 64 & 53 & 144 & 49 \\ 242 & 1 & 11 & 4 \\ 29 & 2 & 38 & 2 \\ 783 & 58 & 58 & 138 \\ 93 & 55 & 182 & 51 \\ 14.5 & 2.1 & 0.5 & 4.7 \\ 114 & 15 & 0 & 38 \\ 9.7 & 0.0 & 0.5 & 0.2 \\ 74 & 0 & 4 & 1 \\ 24.2 & 2.1 & 1.0 & 4.9 \\ 188 & 15 & 4 & 39 \\ 93.0 & 3.0 & 3.0 & 53.0 \\ 709 & 23 & 23 & 404 \\ 0.0 & 0.0 & 0.0 & 0.0 \\ \mathrm{~N} & \mathrm{~N} & \mathrm{~N} & \mathrm{~N} \\ 116 & 10 & 3 & 16 \\ 73.0 & 73.0 & 73.0 & 73.0 \\ 1 & 2 & 3 & 3 \\ \mathrm{E} & \mathrm{B} & \mathrm{E} & \mathrm{B}\end{array}$

\begin{tabular}{rrrrr}
\multicolumn{2}{c}{ Northbound } & \multicolumn{3}{c}{ Southbound } \\
LT TH & RT & LT & TH & RT \\
591 & & 97 & 561 & \\
46 & & 42 & 84 & \\
237 & & 41 & 238 &
\end{tabular}

9.5

57

4.5

0.1

4.7

29

407

69

10

2

417

71

14.0

53

0.4

2

14.4

55

106.0

404

0.0

$\mathrm{N}$

44

47.0

3

C
2.012 .3

$74 \quad 79$

$1.0 \quad 5.8$

0.11 .7

1.17 .5

4348

$\begin{array}{ll}69 & 447\end{array}$

7280

850

910

$77 \quad 497$

8189

2.514 .3

23107

0.32 .0

215

2.816 .3

$25 \quad 122$

3.050 .0

23381

$0.0 \quad 0.0$

$\mathrm{N} \quad \mathrm{N}$

853

$47.0 \quad 47.0$

21

Overall Intersection Results

$\begin{array}{lr}\text { Output Flow (vph) } & 2512 \\ \text { Degree of Sat. (8) } & 107 \\ \text { Tot. Travel (veh-km) } & 1395 \\ \text { Tot. TravTime (veh-h) } & 58 \\ \text { Unif. Delay (veh-h) } & 17.5 \\ \text { Rand. Delay (veh-h) } & 12.7 \\ \text { Total Delay (veh-h) } & 30.2 \\ \text { Avg. Delay (sec/v) } & 43 \\ \text { Unif. Stops (vph) } & 1701 \\ \text { Unif. Stops (8) } & 68 \\ \text { Rand. Stops (vph) } & 329 \\ \text { Rand. Stops (8) } & 13 \\ \text { Total Stops (vph) } & 2031 \\ \text { Total Stops (8) } & 81 \\ \text { Time Full (8) } & 0 \\ \text { Fuel Consumpt. (lit) } & 254 \\ \text { Disutility Index } & 34 \\ \text { Level of Service } & \text { D }\end{array}$ 


\section{TRANSYT-7F Release 10.2 -- Node Output Summary (Detailed) OPTIMIZATION FOR CYCLE LENGTH}

Node Number: 2

Output Flow (vph)

Degree of Sat. (f)

Tot. Travel (veh-km)

Tot.TravTime (veh-h)

Avg. TravTime ( $\mathrm{sec} / \mathrm{v})$

Unif. Delay (veh-h)

Rand. Delay (veh-h)

Total Delay (veh-h)

Avg. Delay (sec/v)

Unif. Stops (vph)

Unif. Stops (8)

Rand. Stops (vph)

Rand. Stops ( $(8)$

Total Stops (vph)

Total Stops $\{8\}$

Unif. MBOQ (veh)

Unif. MBOQ (m/lane)

Rand. MBOQ (veh)

Rand. $M B O Q$ ( $m /$ lane)

Total MBOQ (veh)

Total MBOQ (m/lane)

Q. Capacity (veh)

Q.Capacity (m/lane)

Time Full ( 8 )

Critical Link $(\mathrm{Y} / \mathrm{N})$

Fuel Consumpt. (lit)

EffectiveGreen (sec)

Arrival Type (1-6)

Level of Service

\begin{tabular}{|c|c|c|c|c|c|c|c|c|c|}
\hline \multicolumn{2}{|c|}{ Eastbound } & \multicolumn{3}{|c|}{ Westbound } & \multicolumn{2}{|c|}{ Northbound } & \multicolumn{3}{|c|}{ Southbound } \\
\hline $\mathrm{TH}$ & $\mathrm{RT}$ & LT & $T H$ & $\mathrm{RT}$ & LT & $\mathrm{RT}$ & LT & $\mathrm{TH}$ & RT \\
\hline 331 & & 40 & 253 & & 652 & & & 812 & \\
\hline 45 & & 19 & 53 & & 57 & & & 43 & \\
\hline 133 & & 16 & 101 & & 270 & & & 164 & \\
\hline 6.0 & & 0.8 & 5.2 & & 6.4 & & & 4.2 & \\
\hline 66 & & 74 & 74 & & 35 & & & 18 & \\
\hline 3.2 & & 0.4 & 2.8 & & 0.7 & & & 0.7 & \\
\hline 0.1 & & 0.0 & 0.2 & & 0.3 & & & 0.1 & \\
\hline 3.4 & & 0.5 & 3.1 & & 1.0 & & & 0.9 & \\
\hline 37 & & 46 & 45 & & 5 & & & 4 & \\
\hline 250 & & 32 & 210 & & 127 & & & 101 & \\
\hline 76 & & 81 & 83 & & 20 & & & 13 & \\
\hline 10 & & 2 & 13 & & 13 & & & 9 & \\
\hline 4 & & 8 & 6 & & 3 & & & 2 & \\
\hline 260 & & 35 & 224 & & 141 & & & 110 & \\
\hline 79 & & 89 & 89 & & 22 & & & 14 & \\
\hline 8.7 & & 1.2 & 7.4 & & 3.9 & & & 3.5 & \\
\hline 34 & & 8 & 53 & & 30 & & & 11 & \\
\hline 0.4 & & 0.1 & 0.5 & & 0.5 & & & 0.4 & \\
\hline 2 & & 1 & 4 & & 4 & & & 1 & \\
\hline 9.1 & & 1.3 & 7.9 & & 4.4 & & & 3.8 & \\
\hline 36 & & 9 & 57 & & 34 & & & 12 & \\
\hline 106.0 & & 3.0 & 52.0 & & 53.0 & & & 54.0 & \\
\hline 404 & & 23 & 396 & & 404 & & & 206 & \\
\hline 0.0 & & 0.0 & 0.0 & & 0.0 & & & 0.0 & \\
\hline $\mathbf{N}$ & & $\mathbf{N}$ & $\mathrm{N}$ & & $\mathrm{N}$ & & & $\mathrm{N}$ & \\
\hline 27 & & 3 & 23 & & 31 & & & 20 & \\
\hline 34.0 & & 34.0 & 34.0 & & 86.0 & & & 36.0 & \\
\hline 3 & & 3 & 3 & & 1 & & & 1 & \\
\hline D & & D & D & & A & & & A & \\
\hline
\end{tabular}

Overall Intersection Results

$\begin{array}{lr}\text { Output Flow (vph) } & 2088 \\ \text { Degree of Sat. ( } 8) & 57 \\ \text { Tot. Travel (veh-km) } & 685 \\ \text { Tot.TravTime (veh-h) } & 22 \\ \text { Unif. Delay (veh-h) } & 8.0 \\ \text { Rand. Delay (veh-h) } & 0.9 \\ \text { Total Delay (veh-h) } & 9.0 \\ \text { Avg. Delay (sec/v) } & 15 \\ \text { Unif. Stops (vph) } & 723 \\ \text { Unif. Stops ( } 8) & 35 \\ \text { Rand. Stops (vph) } & 48 \\ \text { Rand. Stops ( } 8) & 2 \\ \text { Total Stops (vph) } & 772 \\ \text { Total Stops ( } 8) & 37 \\ \text { Time Full ( } 8 \text { ) } & 0 \\ \text { Fuel Consumpt. (lit) } & 106 \\ \text { Disutility Index } & 11 \\ \text { Level of Service } & \text { B }\end{array}$


Node Number: 3

Output Flow (vph)

Degree of Sat. ( 8 )

Tot. Travel (veh-km)

Tot.TravTime (veh-h)

Avg.TravTime ( $\mathrm{sec} / \mathrm{v})$

Unif. Delay (veh-h)

Rand. Delay (veh-h)

Total Delay (veh-h)

Avg. Delay (sec/v)

Unif. Stops (vph)

Unif. Stops (\%)

Rand. Stops (vph)

Rand. Stops ( $(8)$

Total Stops (vph)

Total Stops $(8)$

Unif. MBOQ (veh)

Unif. $\mathrm{MBOQ}$ ( $\mathrm{m} /$ lane)

Rand. MBOQ (veh)

Rand. MBOQ ( $\mathrm{m} /$ lane)

Total MBOQ (veh)

Total MBOQ (m/lane)

Q.Capacity (veh)

Q. Capacity (m/lane)

Time Full (o)

Critical Link $(\mathrm{Y} / \mathrm{N})$

Fuel Consumpt. (lit)

EffectiveGreen (sec)

Arrival Type $(1-6)$

Level of Service

\begin{tabular}{|c|c|c|c|c|c|c|c|c|c|}
\hline \multicolumn{2}{|c|}{ Eastbound } & \multicolumn{2}{|c|}{ Westbound } & \multicolumn{3}{|c|}{ Northbound } & \multicolumn{3}{|c|}{ Southbound } \\
\hline LT & $\mathrm{RT}$ & $\mathrm{LT}$ & $\mathrm{RT}$ & $\mathrm{LT}$ & $\mathrm{TH}$ & $\mathrm{RT}$ & $\mathrm{LT}$ & TH & RT \\
\hline 251 & & 164 & & & 651 & & & 584 & \\
\hline 58 & & 41 & & & 63 & & & 27 & \\
\hline 100 & & 65 & & & 133 & & & 157 & \\
\hline 4.3 & & 2.6 & & & 9.4 & & & 4.3 & \\
\hline 61 & & 57 & & & 52 & & & 26 & \\
\hline 1.9 & & 1.1 & & & 6.3 & & & 1.1 & \\
\hline 0.3 & & 0.1 & & & 0.4 & & & 0.0 & \\
\hline 2.2 & & 1.2 & & & 6.8 & & & 1.2 & \\
\hline 32 & " & 28 & & & 37 & & & 7 & \\
\hline 173 & & 105 & & & 451 & & & 278 & \\
\hline 69 & & 64 & & & 69 & & & 48 & \\
\hline 16 & & 8 & & & 16 & & & 1 & \\
\hline 7 & & 6 & & & 3 & & & 1 & \\
\hline 190 & & 114 & & & 468 & & & 279 & \\
\hline 76 & & 70 & & & 72 & & & 48 & \\
\hline 5.7 & & 3.6 & & & 7.3 & & & 10.0 & \\
\hline 46 & & 30 & & & 27 & & & 38 & \\
\hline 0.7 & & 0.3 & & & 0.7 & & & 0.1 & \\
\hline 5 & & 3 & & & 3 & & & 0 & \\
\hline 6.4 & & 3.9 & & & 8.0 & $\therefore$ & & 10.1 & \\
\hline 51 & & 33 & & & 30 & & & 38 & \\
\hline 53.0 & & 53.0 & & & 53.0 & & & 56.0 & \\
\hline 404 & & 404 & & & 202 & & & 213 & \\
\hline 0.0 & & 0.0 & & & 0.0 & & & 0.0 & \\
\hline $\mathrm{N}$ & & $\mathrm{N}$ & & & $\mathrm{N}$ & & & $\mathrm{N}$ & \\
\hline 19 & & 12 & & & 41 & & & 24 & \\
\hline 49.0 & & 49.0 & & & 71.0 & & & 71.0 & \\
\hline 3 & & 3 & & & 1 & & & 1 & \\
\hline C & & $\mathrm{C}$ & & & D & & & A & \\
\hline
\end{tabular}

Overall Intersection Results

Output Flow (vph) 1650

Degree of Sat. (8) 63

Tot. Travel (veh-km) 457

Tot.TravTime (veh-h) 20

Unif. Delay (veh-h) 10.6

Rand. Delay (veh-h) 0.9

Total Delay (veh-h) 11.6

Avg. Delay $(\mathrm{sec} / \mathrm{v}) \quad 25$

Unif. Stops (vph) 1008

Unif. Stops (8) 61

Rand. Stops (vph) 44

Rand. Stops $(8) \quad 3$

Total Stops (vph) 1052

Total Stops (8) 64

Time Eull $(8) \quad 0$

Euel Consumpt. (1it) 98

Disutility Index 14

Level of Service C 
TRANSYT-7F Release 10.2 -- Node Output Summary (Detailed) OPTIMIZATION FOR CYCLE IENGTH

Node Number: 4

Output Flow (vph)

Degree of Sat. (q)

Tot. Travel (veh-km)

Tot.Travime (veh-h)

Avg.TravTime (sec/v)

Unif. Delay (veh-h)

Rand. Delay (veh-h)

Total Delay (veh-h)

Avg. Delay (sec/v)

Unif. Stops (vph)

Unif. Stops (8)

Rand. Stops (vph)

Rand. Stops ( 8 )

Total Stops (vph)

Total Stops ( 8$)$

Unif. MBOQ (veh)

Unif. $M B O Q$ ( $m /$ lane)

Rand. $M B O Q$ (veh)

Rand. $M B O Q$ ( $m /$ lane)

Total MBOQ (veh)

Total MBOQ (m/lane)

Q.Capacity (veh)

Q.Capacity (m/lane)

Time Full (8)

Critical Link ( $Y / N)$

Fuel Consumpt. (lit)

EffectiveGreen (sec)

Arrival Type (1-6)

Level of Service

\begin{tabular}{|c|c|c|c|c|c|}
\hline \multicolumn{2}{|c|}{ Eastbound } & \multicolumn{2}{|c|}{ Westbound } & \multicolumn{2}{|c|}{ Northbound } \\
\hline TH & RT & $\mathrm{TH}$ & $\mathrm{RT}$ & $\operatorname{LT}$ & $\mathrm{RT}$ \\
\hline 2251 & & 1067 & & 461 & \\
\hline 86 & & 95 & & 143 & \\
\hline 1967 & & 428 & & 134 & \\
\hline 55.2 & & 15.7 & & 42.9 & \\
\hline 88 & & 53 & & 335 & \\
\hline 14.7 & & 2.1 & & 13.2 & \\
\hline 1.1 & & 5.0 & & 26.9 & \\
\hline 15.9 & & 7.1 & & 40.2 & \\
\hline 25 & & 24 & & 314 & \\
\hline 2402 & & 481 & & 461 & \\
\hline 107 & & 45 & & 100 & \\
\hline 33 & & 132 & & 466 & \\
\hline 2 & & 13 & & 102 & \\
\hline 2436 & & 613 & & 927 & \\
\hline 109 & & 58 & & 202 & \\
\hline 74.0 & & 10.3 & & 16.3 & \\
\hline 282 & & 38 & & 61 & \\
\hline 1.4 & & 5.3 & & 18.7 & \\
\hline 5 & & 20 & & 71 & \\
\hline 75.4 & & 15.6 & & 35.1 & \\
\hline 287 & & 58 & & 132 & \\
\hline 221.0 & & 105.0 & & 56.0 & \\
\hline 842 & & 400 & & 213 & \\
\hline 0.0 & & 0.0 & & 0.0 & \\
\hline $\mathbf{N}$ & & $\mathrm{N}$ & & $\mathrm{N}$ & \\
\hline 285 & & 71 & & 134 & \\
\hline 86.0 & & 96.0 & & 24.0 & \\
\hline 1 & & 3 & & 1 & \\
\hline C & & $\mathrm{C}$ & & $F$ & \\
\hline
\end{tabular}

Southbound

LT TH RT

154

29

61

3.0

70

1.7

0.0

1.8

42

121

79

5

4

126

83

4.4

15

0.2

1

4.6

16

106.0

404

0.0

$\mathrm{N}$

13

24.0

3

Overall Intersection Results

$\begin{array}{lr}\text { Output Flow (vph) } & 3933 \\ \text { Degree of Sat. (8) } & 143 \\ \text { Tot. Travel (veh-km) } & 2592 \\ \text { Tot.TravTime (veh-h) } & 116 \\ \text { Unif. Delay (veh-h) } & 31.9 \\ \text { Rand. Delay (veh-h) } & 33.2 \\ \text { Total Delay (veh-h) } & 65.1 \\ \text { Avg. Delay (sec/v) } & 59 \\ \text { Unif. Stops (vph) } & 3466 \\ \text { Unif. Stops (8) } & 88 \\ \text { Rand. Stops (vph) } & 637 \\ \text { Rand. Stops ( } 8) & 16 \\ \text { Total Stops (vph) } & 4104 \\ \text { Total Stops (8) } & 104 \\ \text { Time Eull (8) } & 0 \\ \text { Fuel Consumpt. (lit) } & 505 \\ \text { Disutility Index } & 72 \\ \text { Level of Service } & \mathrm{E}\end{array}$


TRANSYT-7F Release 10.2 -- Node Output Summary (Detailed)

OPTIMIZATION FOR CYCLE LENGTH

Node Number: 5

\begin{tabular}{|c|c|c|c|c|c|c|c|c|}
\hline & \multicolumn{3}{|c|}{ Eastbound } & \multicolumn{2}{|c|}{ Westbound } & Northbound & \multicolumn{2}{|c|}{ Southbound } \\
\hline & IT & TH & $\mathrm{RT}$ & $\mathrm{LT}$ & $\mathrm{RT}$ & $\mathrm{TH}$ & $\mathrm{RT}$ & $\mathrm{TH}$ \\
\hline Output Flow (vph) & 407 & 606 & & & 523 & & 1560 & 394 \\
\hline Degree of Sat. (8) & 97 & 43 & & & 140 & & 91 & 97 \\
\hline Tot. Travel (veh-km) & 164 & 219 & & & 447 & & 627 & 158 \\
\hline Tot.TravTime (veh-h) & 14.7 & 11.7 & & & 52.6 & & 29.7 & 12.6 \\
\hline Avg.TravTime (sec/v) & 130 & 69 & & & 371 & & 68 & 115 \\
\hline Unif. Delay (veh-h) & 8.7 & 7.2 & & & 16.0 & & 13.5 & 5.4 \\
\hline Rand. Delay (veh-h) & 2.7 & 0.1 & & & 25.8 & & 3.6 & 4.0 \\
\hline Total Delay (veh-h) & 11.4 & 7.3 & & & 41.8 & & 17.2 & 9.4 \\
\hline Avg. Delay $(\mathrm{sec} / v)$ & 101 & 43 & 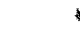 & & 295 & & 39 & 86 \\
\hline Unif. Stops (vph) & 27 & 575 & & & 510 & & 1377 & 385 \\
\hline Unif. Stops $(z)$ & 7 & 95 & & & 100 & & 88 & 98 \\
\hline Rand. Stops (vph) & 70 & 5 & & & 460 & & 100 & 104 \\
\hline Rand. Stops (q) & 18 & 1 & & & 91 & & 7 & 27 \\
\hline Total Stops (vph) & 98 & 581 & & & 970 & & 1477 & 490 \\
\hline Total stops $(\%)$ & 25 & 96 & & & 191 & & 95 & 125 \\
\hline Unif. MBOQ (veh) & 12.1 & 20.7 & & & 26.8 & & 41.2 & 12.9 \\
\hline Unif. MBOQ (m/lane) & 91 & 80 & & & 206 & & 156 & 99 \\
\hline Rand. $M B O Q$ (veh) & 2.8 & 0.2 & & & 18.5 & & 4.0 & 4.2 \\
\hline Rand. $\mathrm{MBOQ}$ (m/lane) & 22 & 1 & & & 141 & & 15 & 32 \\
\hline Total MBOQ (veh) & 15.0 & 20.9 & & & 45.3 & & 45.2 & 17.1 \\
\hline Total MBOQ (m/lane) & 113 & 81 & & & 347 & & 171 & 131 \\
\hline Q. Capacity (veh) & 12.0 & 76.0 & & & $96.0 \quad 12.0$ & & 10.0 & 10.0 \\
\hline Q. Capacity (m/lane) & 91 & 290 & & & 732 & & 38 & 76 \\
\hline Time Full (o) & 49.2 & 0.0 & & & 0.0 & & 54.6 & 20.0 \\
\hline Critical Link $(Y / N)$ & $Y$ & $\mathrm{~N}$ & & & $\mathrm{~N}$ & & $Y$ & $\mathrm{~N}$ \\
\hline Fuel Consumpt. (lit) & 48 & 54 & & & 178 & & 138 & 50 \\
\hline EffectiveGreen (sec) & 58.0 & 48.0 & & & 48.0115 .0 & & 62.0 & 74.0 \\
\hline Arrival Type $(1-6)$ & 6 & 1 & & & 3 & & 3 & 3 \\
\hline Level of Service & F & D & & & A & & D & E \\
\hline
\end{tabular}

Overall Intersection Results

Output Flow (vph) 4000

Degree of Sat. (8) 140

Tot. Travel (veh-km) 2155

Tot.TravTime (veh-h) 130

Unif. Delay (veh-h) 51.2

Rand. Delay (veh-h) 36.3

Total Delay (veh-h) 87.6

Avg. Delay $(\mathrm{sec} / \mathrm{v}) \quad 78$

Unif. Stops (vph) 2952

Unif. Stops (o) 74

Rand. Stops (vph) 742

Rand. Stops (8) 19

Total Stops (vph) 3694

Total stops $(8) \quad 92$

Time Full $(8) \quad 123$

Fuel Consumpt. (lit) 514

Disutility Index 86

Level of Service $E$ 
TRANSYT-7F Release 10.2 -- Node Output Summary (Detailed) OPTIMIZATION FOR CYCLE LENGTH

Node Number: 6

Output Flow (vph)
Degree of Sat. ( 8$)$
Tot. Travel (veh-km)
Tot.TravTime (veh-h)
Avg.TravTime (sec/v)
Unif. Delay (veh-h)
Rand. Delay (veh-h)
Total Delay (veh-h)
Avg. Delay (sec/v)
Unif. Stops (vph)
Unif. Stops ( 8$)$
Rand. Stops (vph)
Rand. Stops ( 8$)$
Total Stops (vph)
Total Stops ( 8$)$
Unif. MBOQ (veh)
Unif. MBOQ (m/lane)
Rand. MBOQ (veh)
Rand. MBOQ (m/lane)
Total MBOQ (veh)
Total MBOQ (m/lane)
Q.Capacity (veh)
Q.Capacity (m/lane)
Time Full (z)
Critical Link (Y/N)
Fuel Consumpt. (lit)
EffectiveGreen (sec)
Arrival Type (1-6)
Level of Service

\begin{tabular}{|c|c|c|c|c|c|c|}
\hline \multicolumn{2}{|c|}{ Eastbound } & \multicolumn{3}{|c|}{ Westbound } & \multicolumn{2}{|c|}{ Northbound } \\
\hline $\mathrm{TH}$ & RT & LT & $\mathrm{TH}$ & RT & $L T$ & $\mathrm{RT}$ \\
\hline 590 & 44 & & 523 & 92 & 763 & \\
\hline 81 & 8 & & 168 & 13 & 51 & \\
\hline 237 & 17 & & 254 & 46 & 329 & \\
\hline 11.9 & 0.6 & & 76.9 & 2.3 & 11.3 & \\
\hline 72 & 56 & & 529 & 90 & 53 & \\
\hline 5.5 & 0.3 & & 26.8 & 1.3 & 4.5 & \\
\hline 1.5 & 0.0 & & 44.9 & 0.0 & 0.2 & \\
\hline 7.1 & 0.3 & & 71.8 & 1.3 & 4.7 & \\
\hline 43 & 27 & & 494 & 54 & 22 & \\
\hline 496 & 28 & & 523 & 83 & 545 & \\
\hline 84 & 64 & & 100 & 91 & 71 & \\
\hline 48 & 1 & & 665 & 0 & 12 & \\
\hline 9 & 3 & & 128 & 1 & 2 & \\
\hline 545 & 29 & & 1188 & 83 & 557 & \\
\hline 93 & 66 & & 228 & 91 & 74 & \\
\hline 16.0 & 1.0 & & 26.3 & 3.0 & 18.5 & \\
\hline 122 & 8 & & 198 & 23 & 72 & \\
\hline 1.9 & 0.0 & & 26.7 & 0.0 & 0.5 & \\
\hline 15 & 0 & & 203 & 0 & 2 & \\
\hline 17.9 & 1.1 & & 52.9 & 3.0 & 19.0 & \\
\hline 137 & 8 & & 401 & 23 & 74 & \\
\hline 50.0 & 3.0 & & 26.0 & 3.0 & 114.0 & \\
\hline 381 & 23 & & 198 & 23 & 434 & \\
\hline 0.0 & 0.0 & & 50.8 & 8.5 & 0.0 & \\
\hline $\mathrm{N}$ & $\mathrm{N}$ & & $Y$ & $\mathrm{~N}$ & $\mathrm{~N}$ & \\
\hline 53 & 3 & & 235 & 10 & 56 & \\
\hline 47.0 & 47.0 & - & 57.0 & 57.0 & 63.0 & \\
\hline 3 & 3 & & 1 & 1 & 1 & \\
\hline D & C & & $E$ & D & C & \\
\hline
\end{tabular}

Southbound

IT TH RT

94920

$230 \quad 3$

$381 \quad 8$

$\begin{array}{ll}196.7 & 0.2\end{array}$

$746 \quad 46$

32.20 .1

$156.8 \quad 0.0$

189.10 .1

$717 \quad 17$

$949 \quad 9$

$100 \quad 50$

$1000 \quad 0$

$106 \quad 2$

$1949 \quad 10$

$206 \quad 52$

$\begin{array}{ll}42.5 & 0.4\end{array}$

$328 \quad 0$

$68.0 \quad 0.0$

$518 \quad 0$

$\begin{array}{ll}110.6 & 0.4\end{array}$

$846 \quad 0$

$40.0 \quad 3.0$

30523

$0.0 \quad 0.0$

$581 \quad 1$

63.063 .0

$3 \quad 3$

Overall Intersection Results

Output Flow (vph) 2981

Degree of Sat. ( $(8) \quad 230$

Tot. Travel (veh-km) 1274

Tot.TravTime (veh-h) 300

Unif. Delay (veh-h) 71.0

Rand. Delay (veh-h) 203.6

Total Delay (veh-h) 274.6

Avg. Delay $(\mathrm{sec} / \mathrm{v}) \quad 331$

Unif. Stops (vph) 2635

Unif. Stops ( 8 ) 88

Rand. Stops (vph) 1727

Rand. Stops (8) 58

Total Stops (vph) 4362

Total Stops $(8) \quad 146$

Time Full $(8) \quad 59$

Fuel Consumpt, (lit) 941

Disutility Index 227

Level of Service $\mathbf{F}$ 
TRANSYT-7F Release 10.2 -- Node Output Summary (Detailed) OPTIMIZATION FOR CYCLE IENGTH

Node Number: 7

\begin{tabular}{|c|c|c|c|c|c|c|c|c|c|c|c|c|}
\hline & \multicolumn{3}{|c|}{ Eastbound } & \multicolumn{3}{|c|}{ Westbound } & \multicolumn{3}{|c|}{ Northbound } & \multicolumn{3}{|c|}{ Southbound } \\
\hline & LT & TH & RT & LT & $\mathrm{TH}$ & $\mathrm{RT}$ & LT & $\mathrm{TH}$ & RT & LT & $\mathrm{TH}$ & RT \\
\hline Output Flow (vph) & 42 & 349 & 49 & 68 & 348 & & & 708 & & & 489 & \\
\hline Degree of Sat. (8) & 15 & 47 & 8 & 23 & 49 & & & 35 & & & 35 & \\
\hline Tot. Travel (veh-km) & 16 & 140 & 19 & 23 & 123 & & & 284 & & & 417 & \\
\hline Tot.TravTime (veh-h) & 0.7 & 5.8 & 0.7 & 0.8 & 4.5 & & & 8.6 & & & 10.5 & \\
\hline Avg.TravTime $(\mathrm{sec} / \mathrm{v})$ & 67 & 60 & 54 & 43 & 47 & & & 44 & & & 77 & \\
\hline Unif. Delay (veh-h) & 0.4 & 2.8 & 0.3 & 0.3 & 1.8 & & & 2.8 & & & 2.1 & \\
\hline Rand, Delay (veh-h) & 0.0 & 0.2 & 0.0 & 0.0 & 0.2 & & & 0.1 & & & 0.0 & \\
\hline Total Delay (veh-h) & 0.4 & 3.0 & 0.3 & 0.3 & 2.0 & & & 2.9 & & & 2.1 & \\
\hline Avg. Delay $(\mathrm{sec} / \mathrm{v})$ & 38 & 31 & 25 & 18 & 21 & & & 15 & & & 16 & \\
\hline Unif. Stops (vph) & 31 & 250 & 29 & 45 & 255 & & & 353 & & & 297 & \\
\hline Unif. Stops $(8)$ & 75 & 72 & 61 & 66 & 73 & & & 50 & & & 61 & \\
\hline Rand. Stops (vph) & 2 & 10 & 1 & 3 & 11 & & & 6 & & & 1 & \\
\hline Rand. Stops $(8)$ & 6 & 4 & 3 & 6 & 4 & & & 1 & & & 1 & \\
\hline Total Stops (vph) & 33 & 261 & 30 & 48 & 266 & & & 360 & & & 298 & \\
\hline Total stops (8) & 81 & 75 & 63 & 72 & 77 & & & 51 & & & 62 & \\
\hline Unif. MBOQ (veh) & 1.1 & 8.6 & 1.1 & 1.1 & 7.6 & & & 12.3 & & & 9.9 & \\
\hline Unif. $M B O Q$ (m/lane) & 8 & 69 & 8 & 8 & 61 & & & 46 & & & 38 & \\
\hline Rand. MBOQ (veh) & 0.1 & 0.4 & 0.0 & 0.1 & 0.4 & & & 0.3 & & & 0.1 & \\
\hline Rand. $\mathrm{MBOQ}$ (m/lane) & 1 & 3 & 0 & 1 & 3 & & & 1 & & & 0 & \\
\hline Total MBOQ (veh) & 1.2 & 9.1 & 1.1 & 1.3 & 8.0 & & & 12.5 & & & 10.0 & \\
\hline Total MBOQ (m/lane) & 9 & 72 & 8 & 9 & 64 & & & 47 & & & 38 & \\
\hline Q. Capacity (veh) & 3.0 & 52.0 & 3.0 & 3.0 & 44.0 & & & 06.0 & & & 13.0 & \\
\hline Q. Capacity (m/lane) & 23 & 396 & 23 & 23 & 335 & & & 404 & & & 431 & \\
\hline Time Full (\&) & 0.0 & 0.0 & 0.0 & 0.0 & 0.0 & & & 0.0 & & & 0.0 & \\
\hline Critical Link $(Y / N)$ & $\mathrm{N}$ & $\mathrm{N}$ & $\mathrm{N}$ & $\mathrm{N}$ & $\mathrm{N}$ & & & $\mathrm{N}$ & & & $\mathrm{N}$ & \\
\hline Fuel Consumpt. (lit) & 3 & 27 & 3 & 4 & 23 & & & 43 & & & 52 & \\
\hline EffectiveGreen (sec) & 51.0 & 51.0 & 51.0 & 51.0 & 51.0 & & & 69.0 & & & 69.0 & \\
\hline Arrival Type $(1-6)$ & 3 & 3 & 3 & 5 & 1 & & & 3 & & & 1 & \\
\hline Level of Service & D & $\mathrm{C}$ & c & $\mathrm{B}$ & $\mathrm{C}$ & & & B & & & $\mathrm{B}$ & \\
\hline
\end{tabular}

Overall Intersection Results

$\begin{array}{lr}\text { Output Flow (vph) } & 2053 \\ \text { Degree of Sat. ( } 8) & 49 \\ \text { Tot. Travel (veh-km) } & 1025 \\ \text { Tot.TravTime (veh-h) } & 31 \\ \text { Unif. Delay (veh-h) } & 10.8 \\ \text { Rand. Delay (veh-h) } & 0.5 \\ \text { Total Delay (veh-h) } & 11.4 \\ \text { Avg. Delay (sec/v) } & 20 \\ \text { Unif. Stops (vph) } & 1262 \\ \text { Unif. Stops ( } 8) & 62 \\ \text { Rand. Stops (vph) } & 37 \\ \text { Rand. Stops ( } 8) & 2 \\ \text { Total Stops (vph) } & 1299 \\ \text { Total Stops ( } 8) & 63 \\ \text { Time Full (z) } & 0 \\ \text { Fuel Consumpt. (lit) } & 157 \\ \text { Disutility Index } & 16 \\ \text { Level of Service } & \mathrm{C}\end{array}$


TRANSYT-7F Release 10.2 -- Node Output Summary (Detailed) OPTIMIZATION FOR CYCLE LENGTH

Node Number: 8

\begin{tabular}{|c|c|c|c|c|c|c|c|c|c|c|c|}
\hline & \multicolumn{3}{|c|}{ Eastbound } & \multicolumn{2}{|c|}{ Westbound } & \multicolumn{3}{|c|}{ Northbound } & \multicolumn{3}{|c|}{ Southbound } \\
\hline & LT & $\mathrm{TH}$ & RT & $\mathrm{TH}$ & RT & $\mathrm{LT}$ & TH & $\mathrm{RT}$ & LT & $\mathrm{TH}$ & $\mathrm{RT}$ \\
\hline Output Flow (vph) & 33 & 489 & 46 & 307 & & 99 & 133 & & & 396 & \\
\hline Degree of Sat. ( 8 ) & 6 & 48 & 13 & 30 & & 42 & 20 & & & 59 & \\
\hline Tot. Travel (veh-km) & 11 & 189 & 18 & 123 & & 41 & 55 & & & 159 & \\
\hline Tot.TravTime (veh-h) & 0.3 & 5.9 & 0.6 & 3.9 & & 1.6 & 2.0 & & & 6.6 & \\
\hline Avg.TravTime (sec/v) & 34 & 43 & 51 & 46 & & 60 & 55 & & & 60 & \\
\hline Unif. Delay (veh-h) & 0.0 & 1.9 & 0.2 & 1.4 & & 0.7 & 0.9 & & & 3.0 & \\
\hline Rand. Delay (veh-h) & 0.0 & 0.2 & 0.0 & 0.0 & & 0.1 & 0.0 & & & 0.4 & \\
\hline Total Delay (veh-h) & 0.0 & 2.1 & 0.2 & 1.4 & & 0.8 & 0.9 & & & 3.4 & \\
\hline Avg. Delay $(\mathrm{sec} / \mathrm{v})$ & 9 & 15 & 22 & 17 & & 30 & 25 & & & 31 & \\
\hline Unif. Stops (vph) & 6 & 221 & 27 & 161 & & 33 & 62 & & & 282 & \\
\hline Unif. Stops (z) & 21 & 45 & 59 & 53 & & 34 & 47 & & & 71 & \\
\hline Rand. Stops (vph) & 0 & 10 & 1 & 5 & & 6 & 2 & & & 17 & \\
\hline Rand. Stops $(8)$ & 3 & 3 & 5 & 2 & & 7 & 2 & & & 5 & \\
\hline Total Stops (vph) & 7 & 232 & 28 & 166 & & 39 & 65 & & & 300 & \\
\hline Total Stops $(8)$ & 24 & 48 & 63 & 55 & & 40 & 49 & & & 76 & \\
\hline Unif. MBOQ (veh) & 0.2 & 7.8 & 0.9 & 5.7 & & 1.2 & 2.2 & & & 9.4 & \\
\hline Unif. MBOQ (m/lane) & 0 & 61 & 8 & 46 & & 8 & 15 & & & 69 & \\
\hline Rand. MBOQ (veh) & 0.0 & 0.4 & 0.1 & 0.2 & & 0.2 & 0.1 & & & 0.7 & \\
\hline Rand. $\mathrm{MBOQ}$ (m/lane) & 0 & 3 & 1 & 2 & & 2 & 1 & & & 5 & \\
\hline Total MBOQ (veh) & 0.3 & 8.3 & 1.0 & 5.9 & & 1.4 & 2.3 & & & 10.1 & \\
\hline Total MBOQ (m/lane) & 0 & 64 & 9 & 48 & & 10 & 16 & & & 74 & \\
\hline Q.Capacity (veh) & 3.0 & 44.0 & 3.0 & 53.0 & & 3.0 & 54.0 & & & 52.0 & \\
\hline Q.Capacity (m/lane) & 23 & 335 & 23 & 404 & & 23 & 411 & & & 396 & \\
\hline Time Full (8) & 0.0 & 0.0 & 0.0 & 0.0 & & 0.0 & 0.0 & & & 0.0 & \\
\hline Critical Link (Y/N) & $\mathrm{N}$ & $\mathbf{N}$ & $\mathrm{N}$ & $\mathrm{N}$ & & N & $\mathbf{N}$ & & & $\mathrm{N}$ & \\
\hline Fuel Consumpt. (lit) & 1 & 28 & 3 & 19 & & 6 & 9 & & & 31 & \\
\hline EffectiveGreen (sec) & 70.0 & 70.0 & 70.0 & 70.0 & & 50.0 & 50.0 & & & 50.0 & \\
\hline Arrival Type $(1-6)$ & 4. & 1 & 3 & 3 & & 5 & 1 & & $\therefore$ & 3 & \\
\hline Level of Service & A & $B$ & C & $\mathrm{B}$ & & $\mathrm{C}$ & $\mathrm{C}$ & & & $\mathrm{C}$ & \\
\hline
\end{tabular}

Overall Intersection Results

$\begin{array}{lr}\text { Output Flow (vph) } & 1503 \\ \text { Degree of Sat. (8) } & 59 \\ \text { Tot. Travel (veh-km) } & 598 \\ \text { Tot. TravTime (veh-h) } & 21 \\ \text { Unif. Delay (veh-h) } & 8.4 \\ \text { Rand. Delay (veh-h) } & 0.8 \\ \text { Total Delay (veh-h) } & 9.2 \\ \text { Avg. Delay (sec/v) } & 22 \\ \text { Unif. Stops (vph) } & 795 \\ \text { Unif. Stops (8) } & 53 \\ \text { Rand. Stops (vph) } & 44 \\ \text { Rand. Stops (8) } & 3 \\ \text { Total Stops (vph) } & 839 \\ \text { Total Stops (8) } & 56 \\ \text { Time Full (8) } & 0 \\ \text { Fuel Consumpt. (lit) } & 100 \\ \text { Disutility Index } & 11 \\ \text { Level of Service } & \text { C }\end{array}$


TRANSYT-7F Release 10.2 -- Node Output Summary (Detailed)

OPTIMIZATION FOR CYCLE LENGTH

Node Number: 9

Output Flow (vph)

Degree of Sat. (\%)

Tot. Travel (veh-km)

Tot.TravTime (veh-h)

Avg.TravTime ( $\mathrm{sec} / \mathrm{v})$

Unif. Delay (veh-h)

Rand. Delay (veh-h)

Total Delay (veh-h)

Avg. Delay (sec/v)

Unif. Stops (vph)

Unif. Stops (z)

Rand. Stops (vph)

Rand. Stops ( $(8)$

Total Stops (vph)

Total Stops $(8)$

Unif. MBOQ (veh)

Unif. $M B O Q$ (m/lane)

Rand. MBOQ (veh)

Rand. $M B O Q$ ( $m /$ lane)

Total MBOQ (veh)

Total MBOQ ( $\mathrm{m} / \mathrm{lane})$

Q. Capacity (veh)

Q.Capacity (m/lane)

Time Full (8)

Critical Link $(Y / N)$

Fuel Consumpt. (lit)

EffectiveGreen (sec)

Arrival Type $(1-6)$

Level of Service

\begin{tabular}{|c|c|c|c|c|c|c|c|c|c|c|}
\hline \multicolumn{2}{|c|}{ Eastbound } & \multicolumn{3}{|c|}{ Westbound } & \multicolumn{3}{|c|}{ Northbound } & \multicolumn{3}{|c|}{ Southbound } \\
\hline $\mathrm{TH}$ & $\mathrm{RT}$ & LT & $\mathrm{TH}$ & RT & $\mathrm{LT}$ & $\mathrm{TH}$ & $\mathrm{RT}$ & LT & $\mathrm{TH}$ & RT \\
\hline 753 & & & 317 & & & 374 & & & 390 & \\
\hline 68 & & & 31 & & & 69 & & & 163 & \\
\hline 302 & & & 250 & & & 150 & & & 161 & \\
\hline 9.6 & & & 5.7 & & & 7.3 & & & 41.7 & \\
\hline 46 & & & 65 & & & 70 & & & 385 & \\
\hline 2.9 & & & 0.6 & & & 3.5 & & & 5.8 & \\
\hline 0.7 & & & 0.0 & & & 0.7 & & & 32.6 & \\
\hline 3.6 & & & 0.7 & & & 4.3 & & & 38.5 & \\
\hline 17 & * & & 8 & & & 41 & & & 355 & \\
\hline 426 & & & 102 & & & 297 & & & 364 & \\
\hline 57 & & & 32 & & & 79 & & & 93 & \\
\hline 25 & & & 4 & & & 26 & & & 497 & \\
\hline 4 & & & 2 & & & 8 & & & 128 & \\
\hline 452 & & & 107 & & & 323 & & & 861 & \\
\hline 61 & & & 35 & & & 87 & & & 221 & \\
\hline 12.9 & & & 3.7 & & & 9.9 & & & 7.5 & \\
\hline 99 & & & 30 & & & 76 & & & 53 & \\
\hline 1.0 & & & 0.2 & & & 1.1 & & & 20.0 & \\
\hline 8 & & & 1 & & & 8 & & & 152 & \\
\hline 13.9 & & & 3.9 & & & 11.0 & & & 27.4 & \\
\hline 107 & & & 31 & & & 84 & & & 205 & \\
\hline 52.0 & & & 98.0 & & & 53.0 & & & 52.0 & \\
\hline 396 & & & 747 & & & 404 & & & 396 & \\
\hline 0.0 & & & 0.0 & & & 0.0 & & & 0.0 & \\
\hline $\mathrm{N}$ & & & $\mathrm{N}$ & & & $\mathrm{N}$ & & & $\mathrm{N}$ & \\
\hline 48 & & & 28 & & & 32 & & & 130 & \\
\hline 79.0 & & & 79.0 & & & 41.0 & & & 41.0 & \\
\hline 3 & & & 1 & & & 3 & & & 1 & \\
\hline B & & & A & & & D & & & F & \\
\hline
\end{tabular}

Overall Intersection Results

Output Flow (vph) 1834

Degree of Sat. ( 8$) \quad 163$

Tot. Travel (veh-km) 865

Tot.TravTime (veh-h) 64

Unif. Delay (veh-h) 13.0

Rand. Delay (veh-h) 34.1

Total Delay (veh-h) 47.2

Avg. Delay $(\mathrm{sec} / \mathrm{v}) \quad 92$

Unif. Stops (vph) 1191

Unif. Stops (8) 65

Rand. Stops (vph) 554

Rand. Stops $(z) \quad 30$

Total Stops (vph) 1745

Total Stops $(8) \quad 95$

Time Full (8) 0

Fuel Consumpt. (lit) 240

Disutility Index 44

Level of Service

44




\title{
Results for Nodes 4, 6 and 9
}

\author{
after changes
}


NOTE: CHANGES: ADDED TURN BAY STORAGE OF 8 TO RIGHT TURN AND MADE LEFT TURN EXCLUSIVE, NO OTHER CHANGES WERE MADE.

TRANSYT-7F Release 10.2 -- Node Output Summary (Detailed)

Node Number: 4

Output Flow (vph)

Degree of Sat. ( 8 )

Tot. Travel (veh-km)

Tot.TravTime (veh-h)

Avg.TravTime $(\mathrm{sec} / \mathrm{v})$

Unif. Delay (veh-h)

Rand. Delay (veh-h)

Total Delay (veh-h)

Avg. Delay (sec/v)

Unif. Stops (vph)

Unif. Stops (o

Rand. Stops (vph)

Rand. Stops ( 8$)$

Total Stops (vph)

Total Stops (q)

Unif. MBOQ (veh)

Unif. MBOQ (m/lane)

Rand. $M B O Q$ (veh)

Rand. $M B O Q$ ( $m /$ lane)

Total MBOQ (veh)

Total MBOQ (m/lane)

Q.Capacity (veh)

Q.Capacity (m/lane)

Time Full (8)

Critical Link ( $Y / N)$

Fuel Consumpt. (Iit)

EffectiveGreen (sec)

Arrival Type $(1-6)$

Level of Service

\begin{tabular}{|c|c|c|c|c|c|c|c|}
\hline \multicolumn{2}{|c|}{ Eastbound } & Westbound & \multicolumn{3}{|c|}{ Northbound } & \multicolumn{2}{|c|}{ Southbound } \\
\hline TH & $\mathrm{RT}$ & LT & RT & $\mathrm{TH}$ & RT & LT & RT \\
\hline 2176 & & 1067 & 197 & 10 & 273 & 154 & \\
\hline 85 & & 94 & 102 & 3 & 99 & 28 & \\
\hline 1967 & & 428 & 74 & 2 & 58 & 61 & \\
\hline 47.0 & & 14.9 & 18.4 & 0.1 & 8.3 & 2.6 & \\
\hline 77 & & 50 & 337 & 41 & 109 & 61 & \\
\hline 6.8 & & 1.6 & 14.2 & 0.0 & 3.1 & 1.3 & \\
\hline 0.8 & & 4.7 & 2.7 & 0.0 & 4.0 & 0.0 & \\
\hline 7.6 & & 6.3 & 16.9 & 0.0 & 7.1 & 1.3 & \\
\hline 12 & : & 21 & 310 & 26 & 94 & 32 & \\
\hline 1836 & & 455 & 197 & 6 & 263 & 120 & \\
\hline 84 & & 43 & 100 & 61 & 96 & 79 & \\
\hline 33 & & 163 & 86 & 0 & 130 & 6 & \\
\hline 2 & & 16 & 44 & 4 & 48 & 5 & \\
\hline 1869 & & 619 & 283 & 6 & 394 & 127 & \\
\hline 86 & & 59 & 144 & 66 & 145 & 83 & \\
\hline 31.5 & & 7.6 & 16.4 & 0.2 & 7.1 & 3.4 & \\
\hline 122 & & 30 & 122 & 0 & 53 & 11 & \\
\hline 1.0 & & 5.0 & 2.7 & 0.0 & 4.0 & 0.2 & \\
\hline 4 & & 19 & 20 & 0 & 31 & 1 & \\
\hline 32.5 & & 12.7 & 19.0 & 0.2 & 11.1 & 3.6 & \\
\hline 126 & & 49 & 142 & 0 & 84 & 12 & \\
\hline 221.0 & & 105.0 & 16.0 & 28.0 & 8.0 & 106.0 & \\
\hline 842 & & 400 & 122 & 213 & 61 & 404 & \\
\hline 0.0 & & 0.0 & 64.0 & 0.0 & 0.0 & 0.0 & \\
\hline $\mathrm{N}$ & & $\mathrm{N}$ & $Y$ & $\mathrm{~N}$ & $\mathrm{~N}$ & $\mathrm{~N}$ & \\
\hline 249 & & 68 & 58 & 0 & 31 & 12 & \\
\hline 63.0 & & 73.0 & 17.0 & 17.0 & 17.0 & 17.0 & \\
\hline 1 & & 3 & 1 & 5 & 3 & 3 & \\
\hline$B$ & & C & $F$ & C & $\mathrm{F}$ & C & \\
\hline
\end{tabular}

Overall Intersection Results

Output Flow (vph) 3877

Degree of Sat. (8) 102

Tot. Travel (veh-km) 2592

Tot.TravTime (veh-h) 91

Unif. Delay (veh-h) 27.2

Rand. Delay (veh-h) 12.4

Total Delay (veh-h) 39.6

Avg. Delay $(\mathrm{sec} / \mathrm{v}) \quad 36$

Unif. Stops (vph) 2879

Unif. Stops (8) 74

Rand. Stops (vph) 420

Rand. Stops ( 8 ) 11

Total Stops (vph) 3299

Total Stops $(8) \quad 85$

Time Full (o) 64

Fuel Consumpt. (lit) 421

Disutility Index 48

Level of Service 
NOTE: EFEECT OF CHANGES MADE IN NODE 4 TO NODE 3

TRANSYT-7F Release 10.2 -- Node Output Summary (Detailed)

Node Number: 3

Output Flow (vph)

Degree of Sat. (8)

Tot. Travel (veh-km)

Tot.TravTime (veh-h)

Avg.TravTime (sec/v)

Unif. Delay (veh-h)

Rand. Delay (veh-h)

Total Delay (veh-h)

Avg. Delay (sec/v)

Unif. Stops (vph)

Unif. Stops (\%)

Rand. Stops (vph)

Rand. Stops (8)

Total Stops (vph)

Total stops (f)

Unif. MBOQ (veh)

Unif. $\mathrm{MBOQ}$ (m/lane)

Rand. MBOQ (veh)

Rand. $\mathrm{MBOQ}$ ( $\mathrm{m} / \mathrm{lane}$

Total MBOQ (veh)

Total MBOQ (m/lane)

Q. Capacity (veh)

Q. Capacity (m/lane)

Time Full (\&)

Critical Link ( $Y / N)$

Euel Consumpt. (1it)

EffectiveGreen (sec)

Arrival Type $(1-6)$

Level of Service

\begin{tabular}{|c|c|c|c|c|c|c|c|c|c|}
\hline \multicolumn{2}{|c|}{ Eastbound } & \multicolumn{3}{|c|}{ Westbound } & \multicolumn{2}{|c|}{ Northbound } & \multicolumn{3}{|c|}{ Southbound } \\
\hline $\mathrm{TH}$ & $\mathrm{RT}$ & LT & TH & $\mathrm{RT}$ & $\mathrm{TH}$ & $\mathrm{RT}$ & LT & $\mathrm{TH}$ & $\mathrm{RT}$ \\
\hline 251 & & & 164 & & 644 & & & 470 & \\
\hline 59 & & & 42 & & 83 & & & 22 & \\
\hline 100 & & & 65 & & 133 & & & 157 & v \\
\hline 3.9 & & & 2.3 & & 21.7 & & & 3.9 & \\
\hline 56 & & & 51 & & 121 & & & 30 & \\
\hline 1.4 & & & 0.8 & & 17.5 & & & 0.8 & \\
\hline 0.4 & & & 0.1 & & 1.5 & & & 0.0 & \\
\hline 1.8 & & & 1.0 & & 19.0 & & & 0.8 & \\
\hline 27 & & & 22 & & 106 & & & 6 & \\
\hline 172 & & & 104 & & 705 & & & 248 & \\
\hline 69 & & & 64 & & 110 & & & 53 & \\
\hline 22 & & & 11 & & 58 & & & 1 & \\
\hline 9 & & & 7 & & 10 & & & $\overline{1}$ & \\
\hline 194 & & & 116 & & 763 & & & 249 & \\
\hline 78 & & & 71 & & 119 & & & 54 & \\
\hline 4.4 & & & 2.8 & & 20.5 & & & 7.0 & \\
\hline 30 & & & 23 & & 80 & & . & 27 & \\
\hline 0.7 & & & 0.4 & & 1.8 & & & 0.1 & \\
\hline 5 & & & 3 & & 7 & & & 0 & \\
\hline 5.1 & & & 3.1 & & 22.3 & & & 7.0 & \\
\hline 35 & & & 26 & & 87 & & & 27 & \\
\hline 53.0 & & & 53.0 & & 53.0 & & & 56.0 & \\
\hline 404 & & & 404 & & 202 & & & 213 & \\
\hline 0.0 & & & 0.0 & & 0.0 & & & 0.0 & \\
\hline $\mathrm{N}$ & & & $\mathrm{N}$ & & $\mathrm{N}$ & & & $\mathrm{N}$ & \\
\hline 18 & & & 11 & & 81 & & & 22 & \\
\hline 36.0 & & & 36.0 & & 54.0 & & & 54.0 & \\
\hline 3 & & & 3 & & 1 & & & 1 & \\
\hline $\mathrm{C}$ & & & c & & $\mathrm{F}$ & & & A & \\
\hline
\end{tabular}

Overall Intersection Results

$\begin{array}{lr}\text { Output Elow (vph) } & 1529 \\ \text { Degree of Sat. (8) } & 83 \\ \text { Tot. Travel (veh-km) } & 457 \\ \text { Tot.TravTime (veh-h) } & 31 \\ \text { Unif. Delay (veh-h) } & 20.7 \\ \text { Rand. Delay (veh-h) } & 2.0 \\ \text { Total Delay (veh-h) } & 22.8 \\ \text { Avg. Delay (sec/v) } & 53 \\ \text { Unif. Stops (vph) } & 1230 \\ \text { Unif. Stops (8) } & 80 \\ \text { Rand. Stops (vph) } & 93 \\ \text { Rand. Stops (8) } & 6 \\ \text { Total Stops (vph) } & 1324 \\ \text { Total Stops (8) } & 87 \\ \text { Time Full (8) } & 0 \\ \text { Fuel Consumpt. (lit) } & 134 \\ \text { Disutility Index } & 24 \\ \text { Level of Service } & \text { D }\end{array}$


NOTE: CHANGES: ADDED $6 \& 4$ TURN BAY STORAGE FOR LEFT \& RIGHT TURN RESPECTIVELY EOR SOUTHBOUND, ADDED LEET TURN BAY STORAGE OF 8 VEHILCES FOR WESTBOUND

TRANSYT-7F Release 10.2 -- Node Output Summary (Detailed)

Node Number: 6

Output Elow (vph)

Degree of Sat. (8)

Tot. Travel (veh-km)

Tot.TravTime (veh-h)

Avg.TravTime (sec/v)

Unif. Delay (veh-h)

Rand. Delay (veh-h)

Total Delay (veh-h)

Avg. Delay (sec/v)

Unif. Stops (vph)

Unif. Stops (8)

Rand, Stops (vph)

Rand. Stops (8)

Total Stops (vph)

Total Stops (z)

Unif. MBOQ (veh)

Unif. MBOQ (m/lane)

Rand. MBOQ (veh)

Rand. MBOQ (m/lane)

Total MBOQ (veh)

Total MBOQ (m/lane)

Q. Capacity (veh)

Q. Capacity (m/lane)

Time Full ( 8 )

Critical Link $(Y / N)$

Fuel Consumpt. (Iit)

EffectiveGreen (sec)

Arrival Type (1-6)

Level of Service

\begin{tabular}{rrrrr}
\multicolumn{3}{c}{ Eastbound } & \multicolumn{3}{c}{ Westbound } \\
LT TH & RT & LT & TH & RT \\
590 & 44 & 239 & 266 & \\
85 & 8 & 100 & 40 \\
237 & 17 & 92 & 75 \\
11.3 & 0.6 & 10.9 & 4.3 \\
69 & 52 & 164 & 58 \\
4.5 & 0.2 & 6.2 & 2.6 \\
2.0 & 0.0 & 2.8 & 0.1 \\
6.6 & 0.2 & 9.0 & 2.7 \\
40 & 23 & 136 & 37 \\
506 & 28 & 187 & 189 \\
86 & 66 & 79 & 71 \\
78 & 1 & 93 & 10 \\
14 & 4 & 40 & 4 \\
584 & 30 & 281 & 200 \\
99 & 69 & 118 & 76 \\
12.6 & 0.8 & 8.1 & 5.0 \\
99 & 8 & 61 & 38 \\
2.4 & 0.0 & 2.9 & 0.3 \\
18 & 0 & 22 & 2 \\
15.0 & 0.9 & 11.0 & 5.3 \\
117 & 8 & 83 & 40 \\
49.0 & 3.0 & 8.0 & 38.0 \\
373 & 23 & 61 & 290 \\
0.0 & 0.0 & 53.0 & 0.0 \\
$\mathrm{~N}$ & $\mathrm{~N}$ & $\mathrm{Y}$ & $\mathrm{N}$ \\
52 & 3 & 38 & 19 \\
33.0 & 33.0 & 43.0 & 43.0 \\
3 & 3 & 4 & 3 \\
$\mathrm{D}$ & $\mathrm{C}$ & $\mathrm{F}$ & $\mathrm{D}$
\end{tabular}

Northbound Southbound LT TH RT LT TH RT

770

49

329

10.0

46

3.2

0.2

3.4

16

543

71

14

2

558

73

14.2

53

0.5

2

14.7

55

114.0

434

0.0

N

53

47.0

I $\begin{array}{lll}344 & 100 & 100\end{array}$

$\begin{array}{lll}116 & 11 & 13\end{array}$

$\begin{array}{lll}138 & 40 & 40\end{array}$

$\begin{array}{lll}16.8 & 1.2 & 1.2\end{array}$

$\begin{array}{lll}176 & 44 & 44\end{array}$

$\begin{array}{lll}4.2 & 0.4 & 0.4\end{array}$

$\begin{array}{lll}9.9 & 0.0 & 0.0\end{array}$

$\begin{array}{lll}14.1 & 0.4 & 0.4\end{array}$

$147 \quad 15 \quad 15$.

$344 \quad 52 \quad 53$

$100 \quad 53 \quad 54$

$\begin{array}{lll}276 & 2 & 2\end{array}$

8133

$620 \quad 54 \quad 56$

$\begin{array}{lll}181 & 55 & 57\end{array}$

$\begin{array}{lll}7.3 & 1.5 & 1.5\end{array}$

$\begin{array}{lll}53 & 15 & 15\end{array}$

$\begin{array}{lll}8.5 & 0.1 & 0.1\end{array}$

$\begin{array}{lll}65 & 0 & 1\end{array}$

$\begin{array}{lll}15.8 & 1.6 & 1.6\end{array}$

$\begin{array}{lll}118 & 15 & 16\end{array}$

$6.0 \quad 53.0 \quad 4.0$

$\begin{array}{lll}46 & 404 & 30\end{array}$

$\begin{array}{lll}0.0 & 0.0 & 0.0\end{array}$

$\mathrm{N} \quad \mathrm{N} \quad \mathrm{N}$

6066

$\begin{array}{llll}47.0 & 47.0 & 47.0\end{array}$

Overall Intersection Results

Output Flow (vph) 2453

Degree of Sat. ( $(8) \quad 116$

Tot. Travel (veh-km) 971

Tot.TravTime (veh-h) 56

Unif. Delay (veh-h) 21.9

Rand. Delay (veh-h) 15.2

Total Delay (veh-h) 37.1

Avg. Delay (sec/v) 54

Unif. Stops (vph) 1906

Unif. Stops (8) 78

Rand. Stops (vph) 478

Rand. Stops ( 8 ) 20

Total Stops (vph) 2384

Total Stops $(8) \quad 97$

Time Full $(8) \quad 52$

Fuel Consumpt. (lit) 238

Disutility Index 41

Level of Service 
NOTE: EFFECT OF CHANGES MADE IN NODE 6 TO NODE 5

TRANSYT-7F Release 10.2 -- Node Output Summary (Detailed)

Node Number: 5

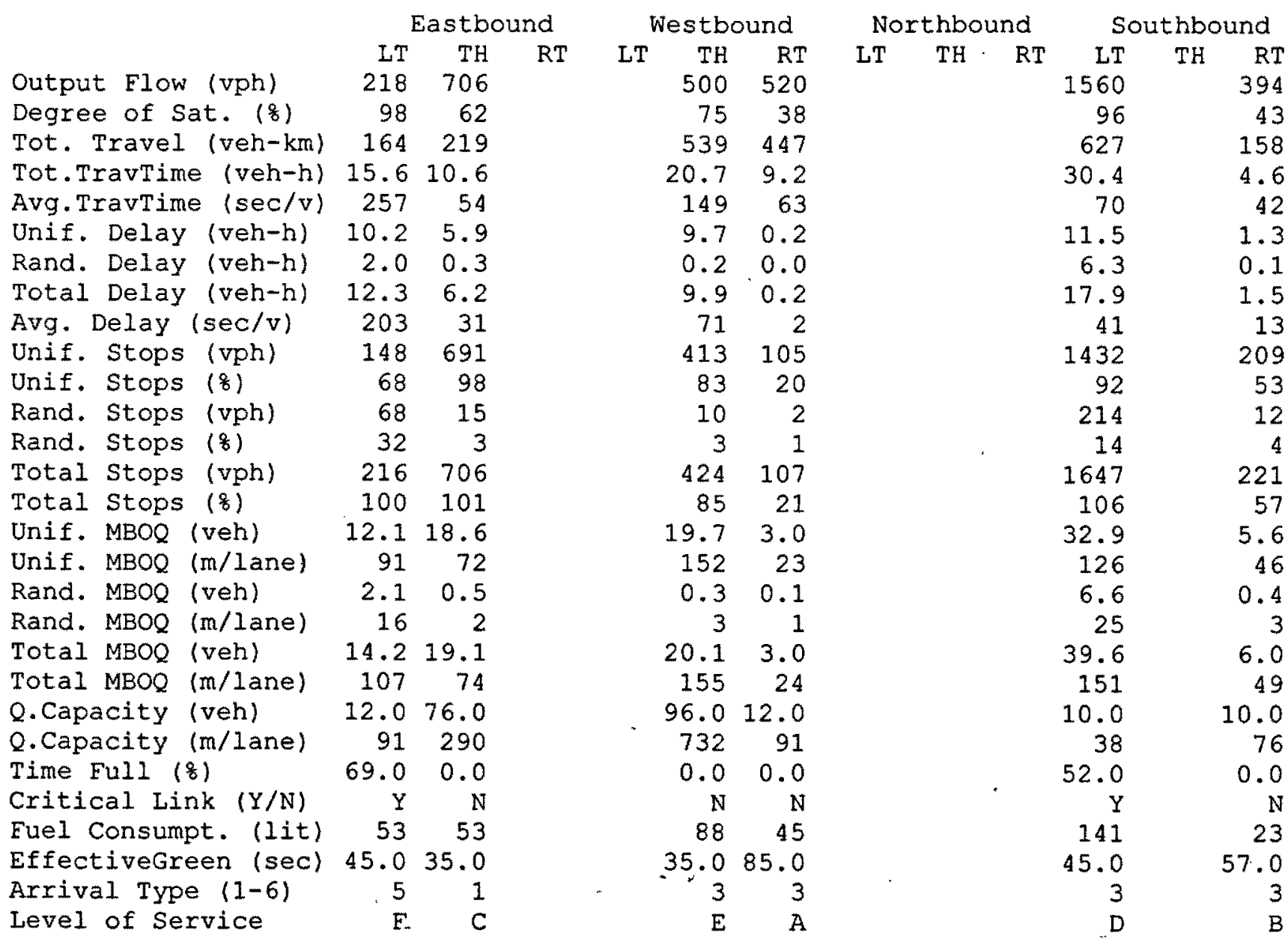

Overall. Intersection Results

Output Flow (vph) 3898

Degree of Sat. ( 8 ) 98

Tot. Travel (veh-km) 2155

Tot.TravTime (veh-h) 91

Unif. Delay (veh-h) 39.0

Rand. Delay (veh-h) 9.1

Total Delay (veh-h) 48.2

Avg. Delay (sec/v) 44

Unif. Stops (vph) 3000

Unif. Stops (8) 77

Rand. Stops (vph) 323

Rand. Stops (8) 8

Total Stops (vph) 3324

Total Stops (8) 85

Time Full (of) 121

Fuel Consumpt. (lit) 406

Disutility Index 55

Level of Service D 
NOTE: CHANGES IN LANE CONEIGURATION FOR NODE 9. ADDED EXCLUSTVE LEFT TURN SOR SOUTHBOUND.

TRANSYT-7F Release 10.2 -- Node Output Summary (Detailed)

Node Number:

9

Output Flow (vph)

Degree of Sat. (s)

Tot. Travel (veh-km)

Tot.TravTime (veh-h)

Avg.TravTime (sec/v)

Unif. Delay (veh-h)

Rand. Delay (veh-h)

Total Delay (veh-h)

Avg. Delay (sec/v)

Unif. Stops (vph)

Unif. Stops (z)

Rand. Stops (vph)

Rand. Stops (o)

Total Stops (vph)

Total Stops (z)

Unif. MBOQ (veh)

Unif. MBOQ (m/lane)

Rand. $M B O Q$ (veh)

Rand. $M B O Q$ ( $m /$ lane)

Total MBOQ (veh)

"Total MBOQ (m/lane)

Q. Capacity (veh)

Q. Capacity (m/lane)

Time Full ( $(8)$

Critical Link ( $Y / N)$

Fuel Consumpt. (lit)

EffectiveGreen (sec)

Arrival Type (1-6)

Level of Service

\begin{tabular}{|c|c|c|c|c|c|c|c|c|c|c|}
\hline \multicolumn{2}{|c|}{ Eastbound } & \multicolumn{3}{|c|}{ Westbound } & \multicolumn{3}{|c|}{ Northbound } & \multicolumn{3}{|c|}{ Southbound } \\
\hline LT & RT & LT & $\mathrm{TH}$ & $\mathrm{RT}$ & $\mathrm{LT}$ & $\mathrm{TH}$ & $\mathrm{RT}$ & LT & $\mathrm{TH}$ & $\mathrm{RT}$ \\
\hline 753 & & & 319 & & & 374 & & 166 & 224 & \\
\hline 67 & & & 30 & & & 71 & & 114 & 39 & \\
\hline 302 & & & 250 & & & 150 & & 68 & 92 & \\
\hline 9.6 & & & 5.7 & & & 7.8 & & 9.4 & 4.3 & \\
\hline 46 & & & 64 & & & 75 & & 204 & 70 & \\
\hline 2.9 & & & 0.6 & & & 4.0 & & 3.0 & 2.3 & \\
\hline 0.6 & & & 0.0 & & & 0.8 & & 5.0 & 0.1 & \\
\hline 3.6 & & & 0.7 & & & 4.8 & & 8.0 & 2.5 & \\
\hline 17 & * & & 8 & & & 46 & & 174 & 40 & \\
\hline 415 & & & 98 & & & 303 & & 153 & 171 & \\
\hline 55 & & & 31 & & & 81 & & 93 & 76 & \\
\hline 22 & & & 4 & & & 27 & & 101 & 7 & \\
\hline 4 & & & 2 & & & 8 & & 62 & 4 & \\
\hline 437 & & & 102 & & & 331 & & 255 & 178 & \\
\hline 59 & & & 33 & & & 89 & & 154 & 80 & \\
\hline 13.3 & & & 3.8 & & & 10.8 & & 3.8 & 6.5 & \\
\hline 99 & & & 30 & & & 84 & & 30 & 53 & \\
\hline 1.0 & & & 0.2 & & & 1.2 & & 4.4 & 0.3 & \\
\hline 7 & & & 1 & & & 9 & & 33 & 2 & \\
\hline 14.3 & & & 3.9 & & & 12.0 & & 8.2 & 6.8 & \\
\hline 106 & & & 31 & & & 93 & & 63 & 55 & \\
\hline 52.0 & & & 98.0 & & & 53.0 & & 53.0 & 53.0 & \\
\hline 396 & & & 747 & & & 404 & & 404 & 404 & \\
\hline 0.0 & & & 0.0 & & & 0.0 & & 0.0 & 0.0 & \\
\hline $\mathrm{N}$ & & & $\mathrm{N}$ & & & $\mathrm{N}$ & & $\mathrm{N}$ & $\mathrm{N}$ & \\
\hline 48 & & & 28 & & & 34 & & 32 & 19 & \\
\hline 87.0 & & & 87.0 & & & 43.0 & & 43.0 & 43.0 & \\
\hline 3 & & & 1 & & & 3 & & 4 & 3 & \\
\hline$B$ & & & A & & & D & & $\mathrm{F}$ & D & \\
\hline
\end{tabular}

Overall Intersection Results

$\begin{array}{lr}\text { Output Flow (vph) } & 1836 \\ \text { Degree of Sat. ( } 8 \text { ) } & 114 \\ \text { Tot. Travel (veh-km) } & 865 \\ \text { Tot.TravTime (veh-h) } & 37 \\ \text { Unif. Delay (veh-h) } & 13.0 \\ \text { Rand. Delay (veh-h) } & 6.6 \\ \text { Total Delay (veh-h) } & 19.6 \\ \text { Avg. Delay (sec/v) } & 38 \\ \text { Unif. Stops (vph) } & 1141 \\ \text { Unif. Stops ( } 8) & 62 \\ \text { Rand. Stops (vph) } & 163 \\ \text { Rand. Stops ( } 8) & 9 \\ \text { Total Stops (vph) } & 1305 \\ \text { Total Stops ( } 8) & 71 \\ \text { Time Full ( } 8 \text { ) } & 0 \\ \text { Fuel Consumpt. (lit) } & 162 \\ \text { Disutility Index } & 22 \\ \text { Level of Service } & \text { D }\end{array}$




\section{Appendix B}

\section{Synchro Report}




\section{Lane Group}

Lane Configurations

Volume (vph)

satd. Flow (prot)

Flt Permitted

Satd. Flow (perm)

Satd. Flow (RTOR)

Lane Group Flow (vph)

Turn Type

Protected Phases

Permitted Phases

Total Split (s)

Total Lost Time (s)

Act Effct Green (s)

Actuated g/C Ratio

v/c Ratio

Control Delay

Queue Delay

Total Delay

LOS

Approach Delay

Approach LOS

Stops (vph)

Euel Used (I)

CO Emissions ( $\mathrm{g} / \mathrm{hr}$ )

NOx Emissions (g/hr)

VOC Emissions (g/hr)

Dilemma Vehicles (\#)

Queue Length 50th (m)

Queue Length 95th (m)

Internal Link Dist (m)

Turn Bay Length (m)

Base Capacity (vph)

Starvation Cap Reductn

Spillback Cap Reductn

Storage Cap Reductn

Reduced v/c Ratio

Intersection Summary

Cycle Length: 125

Actuated Cycle Length: 125

Offset: $0(08)$, Referenced to phase 2: EBWB and 6:, Start of Green

Control Type: Pretimed

Maximum v/c Ratio: 1.82

Intersection Signal Delay: 255.9

Intersection Capacity Utilization 114.88

Analysis Period.(min) 15

$\sim$ Volume exceeds capacity, queue is theoretically infinite.

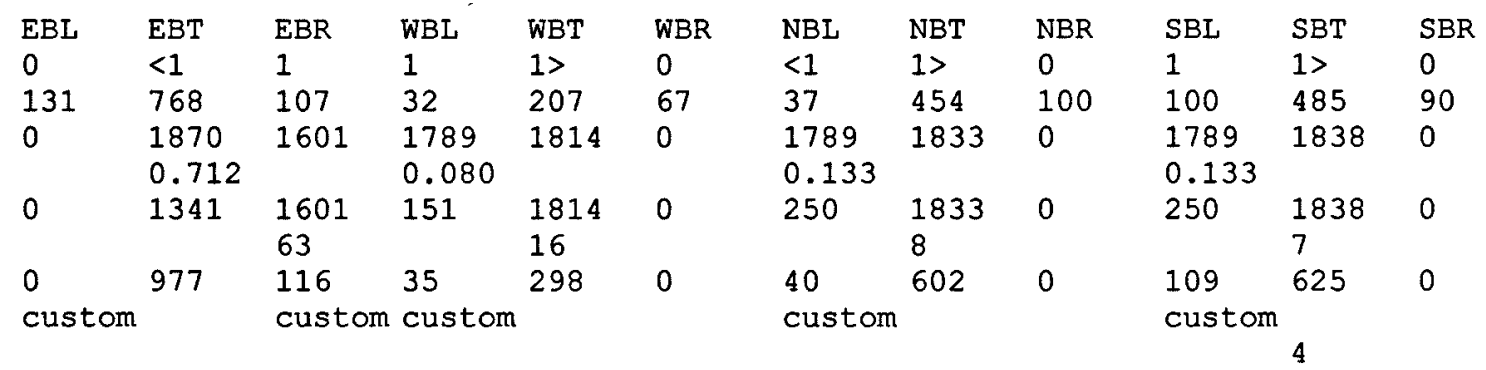

$\begin{array}{lllll}2 & 2 & 2 & 2 & 2 \\ 55.0 & 55.0 & 55.0 & 55.0 & 55.0 \\ 5.0 & 5.0 & 5.0 & 5.0 & 5.0 \\ & 50.0 & 50.0 & 50.0 & 50.0 \\ & 0.40 & 0.40 & 0.40 & 0.40 \\ & 1.82 & 0.17 & 0.58 & 0.41 \\ & 403.7 & 12.3 & 71.1 & 27.4 \\ & 0.0 & 0.0 & 0.0 & 0.0 \\ 403.7 & 12.3 & 71.1 & 27.4 \\ \mathrm{~F} & \mathrm{~B} & \mathrm{E} & \mathrm{C} \\ 362.1 & & & 32.0 \\ \mathrm{~F} & & & \mathrm{C} \\ 600 & 32 & 26 & 182 \\ & 364 & 11 & 3 & 13 \\ 6738 & 195 & 50 & 246 \\ 1311 & 38 & 10 & 48 \\ 1563 & 45 & 12 & 57 \\ 0 & 0 & 0 & 0 \\ & \sim 362.3 & 8.0 & 6.6 & 48.9 \\ & \# 439.4 & 20.1 & \# 24.4 & 72.8 \\ 778.0 & & & 146.4 \\ & & & & \\ & 536 & 678 & 60 & 735 \\ 0 & 0 & 0 & 0 \\ 0 & 0 & 0 & 0 \\ 0 & 0 & 0 & 0 \\ 1.82 & 0.17 & 0.58 & 0.41\end{array}$

$1 \quad 1$

$\begin{array}{llllll}35.0 & 35.0 & 0.0 & 35.0 & 35.0 & 0.0\end{array}$

$\begin{array}{llllll}5.0 & 5.0 & 4.0 & 5.0 & 4.0 & 4.0\end{array}$

$30.0 \quad 30.0 \quad 30.0 \quad 31.0$

$\begin{array}{llll}0.24 & 0.24 & 0.24 & 0.25\end{array}$

$\begin{array}{llll}0.67 & 1.35 & 1.82 & 1.36\end{array}$

$93.0 \quad 208.8 \quad 454.5 \quad 210.6$

$\begin{array}{llll}0.0 & 0.0 & 0.0 & 0.0\end{array}$

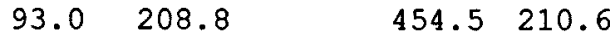

F $\quad \mathrm{F}$

201.6

F $\quad F$

246.8

E

$\begin{array}{llll}32 & 425 & 65 & 441\end{array}$

$3 \quad 101 \quad 40 \quad 125$

$\begin{array}{llll}64 & 1863 & 746 & 2308\end{array}$

$\begin{array}{llll}13 & 362 & 145 & 449\end{array}$

$\begin{array}{llll}15 & 432 & 173 & 535\end{array}$

$0 \quad 0$

$9.0 \sim 193.4$

$\sim 40.4 \sim 201.6$

$\begin{array}{ll}\sim 40.4 & \sim 201.6 \\ \# 77.2 & \# 272.2\end{array}$

71.0

391.0

$\begin{array}{ll}60 & 446 \\ 0 & 0 \\ 0 & 0 \\ 0 & 0 \\ 0.67 & 1.35\end{array}$

$\begin{array}{ll}60 & 461 \\ 0 & 0 \\ 0 & 0 \\ 0 & 0 \\ 1.82 & 1.36\end{array}$

Intersection LOS:

ICU Level of Service H 
Lane Group

Lane Configurations

Volume (vph)

Satd. Flow (prot)

Elt Permitted

Satd. Flow (perm)

Satd. Flow (RTOR)

Lane Group Flow (vph)

Turn Type

Protected Phases

Permitted Phases

Total Split (s)

Total Lost Time (s)

Act Effct Green (s)

Actuated $\mathrm{g} / \mathrm{C}$ Ratio

$v / c$ Ratio

Control Delay

Queue Delay

Total Delay

LOS

Approach Delay

Approach LOS

Stops (vph)

Fuel Used(1)

Co Emissions $(g / h r)$

Nox Emissions $(\mathrm{g} / \mathrm{hr})$

VOC Emissions $(\mathrm{g} / \mathrm{hr})$

Dilemma Vehicles (\#)

Quele Length 50 th (m)

Queue Length $95 \mathrm{th}$ (m)

Internal Link'Dist (m)

Turn Bay Length (m)

Base Capacity (vph)

Starvation Cap Reductn

Spillback Cap Reductn

Storage Cap Reductn

Reduced v/c Ratio

Intersection Summary

Cycle Length: 90

Actuated Cycle Length: 90

Offset: $0(0 \%)$, Referenced to phase 2:EBWB and $6:$, Start of Green

Control Type: Pretimed

Maximum v/c Ratio: 0.69

Intersection Signal Delay: 17.5

Intersection Capacity Utilization 98.38

Analysis Period (min) 15

\# 95th percentile volume exceeds capacity, queue may be longer. Queue shown is maximum after two cycles.

\begin{tabular}{|c|c|c|c|c|c|c|c|c|c|c|c|}
\hline EBL & EBT & EBR & WBL & WBT & WBR & NBL & NBT & NBR & SBL & $S B T$ & SBR \\
\hline 0 & $\langle 2\rangle$ & 0 & 1 & $1>$ & 0 & 0 & $\langle 1\rangle$ & 0 & 0 & $\langle 2\rangle$ & 0 \\
\hline 59 & 243 & 29 & 40 & 197 & 66 & 36 & 537 & 79 & 102 & 606 & 98 \\
\hline 0 & $\begin{array}{l}3500 \\
0.683\end{array}$ & 0 & $\begin{array}{l}1789 \\
0.450\end{array}$ & 1812 & 0 & 0 & $\begin{array}{l}1848 \\
0.922\end{array}$ & 0 & 0 & $\begin{array}{l}3493 \\
0.763\end{array}$ & 0 \\
\hline 0 & $\begin{array}{l}2412 \\
10\end{array}$ & 0 & 848 & $\begin{array}{l}1812 \\
17\end{array}$ & 0 & 0 & $\begin{array}{l}1709 \\
17\end{array}$ & 0 & 0 & $\begin{array}{l}2681 \\
36\end{array}$ & 0 \\
\hline $\begin{array}{l}0 \\
\text { custom }\end{array}$ & 360 & 0 & $\begin{array}{l}43 \\
\text { custom }\end{array}$ & 286 & 0 & $\begin{array}{l}0 \\
\text { cust }\end{array}$ & 709 & 0 & $\begin{array}{l}0 \\
\text { cus }\end{array}$ & 877 & 0 \\
\hline
\end{tabular}

\begin{tabular}{|c|c|c|c|c|}
\hline 2 & 2 & & & 2 \\
\hline 25.0 & 25.0 & 0.0 & 25.0 & 25.0 \\
\hline 5.0 & 5.0 & 4.0 & 5.0 & 5.0 \\
\hline & 0.22 & & 0.22 & 0.22 \\
\hline & 0.66 & ' & 0.23 & 0.69 \\
\hline & 37.7 & & 32.5 & 39.9 \\
\hline & 0.0 & 8 & 0.0 & 0.0 \\
\hline & 37.7 & & 32.5 & 39.9 \\
\hline & D & & C & D \\
\hline & 37.7 & & & 38.9 \\
\hline & D & & & D \\
\hline & 288 & & 33 & 223 \\
\hline & 18 & & 2 & 14 \\
\hline & 330 & & 36 & 268 \\
\hline & 64 & & 7 & 52 \\
\hline & 76 & & 8 & 62 \\
\hline & 0 & & 0 & 0 \\
\hline & 29.2 & & 6.1 & 42.9 \\
\hline & 44.0 & & 15.3 & $\# 69.8$ \\
\hline & 92.3 & & & 90.9 \\
\hline & 544 & & 188 & 416 \\
\hline & 0 & & 0 & 0 \\
\hline & 0 & & 0 & 0 \\
\hline & 0 & & 0 & 0 \\
\hline & 0.66 & & 0.23 & 0.69 \\
\hline
\end{tabular}

\section{1}

$\begin{array}{llll}0.0 & 65.0 & 65.0 & 0.0\end{array}$

11

$\begin{array}{llll}4.0 & 5.0 & 5.0 & 4.0\end{array}$

65.0

65.

0.0

60.0

0.67

0.62

11.2

0.0

11.2

$\mathrm{B}$

11.2

B

346

39

726

141

168

0

59.6

91.3

391.0

5.0

60.0

0.67

0.49

6.3

0.0

6.3

A

6.3

A

379

27

495

96

115

0

21.4

24.8

180.0

1145

1799

0

0

0

0.62

0

0

0.49
Intersection LOS: B ICU Level of Service F 
Lane Group

Lane Configurations

Volume (vph)

Satd. Flow (prot)

Flt Permitted

Satd. Flow (perm)

satd. Flow (RTOR)

Lane Group Elow (vph)

Turn Type

protected Phases

Permitted Phases

Total Split (s)

Total Lost Time (s)

Act Effct Green (s)

Actuated $\mathrm{g} / \mathrm{C}$ Ratio

v/c Ratio

Control Delay

Queue Delay

Total Delay

LOS

Approach Delay

Approach LOS

Stops (vph)

Fuel Used (1)

Co Emissions ( $g / h r)$

NOx Emissions (g/hr)

VOC Emissions (g/hr)

Dilemma Vehicles (\#)

Queue Length 50th (m)

Queve Length 95th (m)

Internal Link Dist (m)

\begin{tabular}{|c|c|c|c|c|c|c|c|c|c|c|c|}
\hline EBL & EBT & EBR & WBL & WBT & WBR & NBL & NBT & NBR & SBL & SBT & SBR \\
\hline 0 & $\langle 1\rangle$ & 0 & 0 & $\langle 1\rangle$ & 0 & 0 & $\langle 2\rangle$ & 0 & 0 & $\langle 2\rangle$ & 0 \\
\hline 68 & 118 & 65 & 49 & 47 & 68 & 76 & 496 & 80 & 10 & 692 & 39 \\
\hline 0 & $\begin{array}{l}1794 \\
0.876\end{array}$ & 0 & 0 & $\begin{array}{l}1751 \\
0.852\end{array}$ & 0 & 0 & $\begin{array}{l}3493 \\
0.750\end{array}$ & 0 & 0 & $\begin{array}{l}3546 \\
0.944\end{array}$ & 0 \\
\hline 0 & $\begin{array}{l}1592 \\
21\end{array}$ & 0 & 0 & $\begin{array}{l}1515 \\
43\end{array}$ & 0 & 0 & $\begin{array}{l}2636 \\
27\end{array}$ & 0 & 0 & $\begin{array}{l}3351 \\
10\end{array}$ & 0 \\
\hline 0 & 273 & 0 & 0 & 178 & 0 & 0 & 709 & 0 & 0 & 805 & 0 \\
\hline
\end{tabular}

custom custom

\begin{tabular}{|c|c|c|c|c|}
\hline 2 & 2 & & 2 & 2 \\
\hline 36.0 & 36.0 & 0.0 & 36.0 & 36.0 \\
\hline 5.0 & 5.0 & 4.0 & 5.0 & 5.0 \\
\hline & 31.0 & & & 31.0 \\
\hline & 0.34 & & & 0.34 \\
\hline & 0.49 & & & 0.32 \\
\hline & 24.9 & & & 18.2 \\
\hline & 0.0 & & & 0.0 \\
\hline & 24.9 & & & 18.2 \\
\hline & C & & & B \\
\hline & 24.9 & & & 18.2 \\
\hline & $\mathrm{C}$ & & & B \\
\hline & 178 & & & 89 \\
\hline & $10^{\circ}$ & & & 6 \\
\hline & 179 & & & 117 \\
\hline & 35 & & & 23 \\
\hline & 42 & & & 27 \\
\hline & 0 & & & 0 \\
\hline & 33.7 & & & 16.6 \\
\hline & 56.5 & & & 32.7 \\
\hline & 63.7 & & & 132.9 \\
\hline & 562 & & & 550 \\
\hline & 0 & & & 0 \\
\hline & 0 & & & 0 \\
\hline & 0. & & & 0 \\
\hline & 0.49 & & & 0.32 \\
\hline
\end{tabular}

custorn

custom

Turn Bay Length (m)

Base Capacity (vph)

Starvation Cap Reductn

Spillback Cap Reductn

Storage Cap Reductn

Reduced $v / c$ Ratio

Intersection Summary

Cycle Length: 90

Actuated Cycle Length: 90

Offset: $0(08)$, Referenced to phase $2:$ EBWB and $6:$, start of Green

Control Type: Pretimed

Maximum v/c Ratio: 0.49

Intersection Signal Delay: 13.8

Intersection Capacity Utilization 69.58

$\begin{array}{lll}1 & 1 & \\ 54.0 & 54.0 & 0.0 \\ 5.0 & 5.0 & 4.0 \\ & 49.0 & \\ & 0.54 & \\ & 0.49 \\ & 9.3 \\ & 0.0 \\ 9.3 & \\ & A \\ 9.3 \\ \text { A } \\ 332 \\ 24 \\ 435 \\ 85 \\ 101 \\ 0 \\ 24.0 \\ 30.3 \\ 180.0 \\ \\ 1447 \\ 0 \\ 0 \\ 0 \\ 0.49 \\ \end{array}$

$\begin{array}{lll}1 & 1 & \\ 54.0 & 54.0 & 0.0 \\ 5.0 & 5.0 & 4.0 \\ & 49.0 & \\ & 0.54 \\ & 0.44 \\ & 13.1 \\ & 0.0 \\ & 13.1 \\ 8 & \\ & 13.1 \\ & 8 \\ 414 & \\ 30 & \\ & 557 \\ 108 \\ 129 \\ 0 \\ 40.6 \\ 54.0 \\ 189.0 \\ 1829 \\ 0 \\ 0 \\ 0 \\ 0.44 \\ \end{array}$

Analysis period (min) 15 


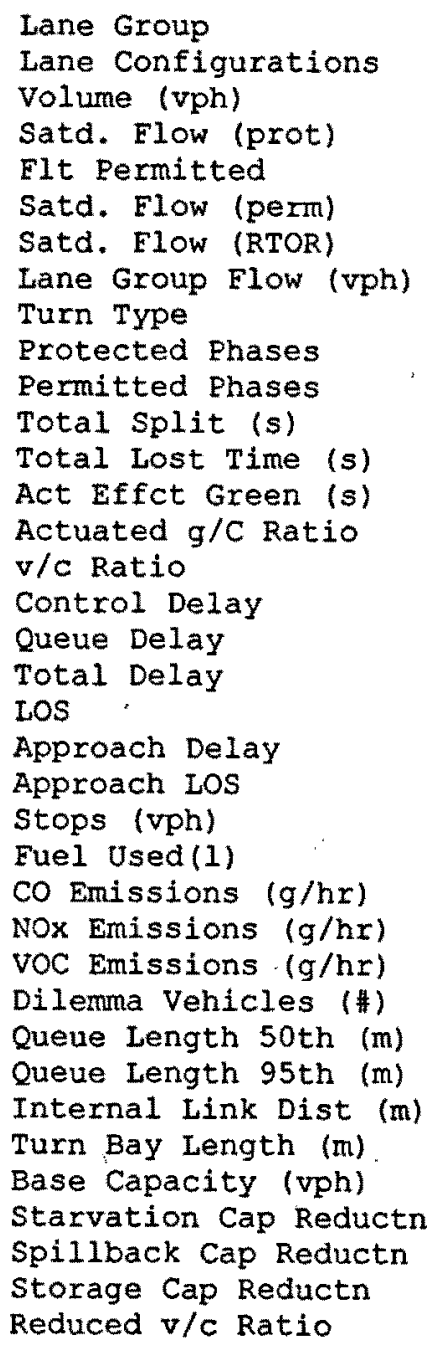

Intersection Summary Cycle Length: 162

Actuated Cycle Length: 162

Offset: $0(0 \%)$, Referenced to phase 2:EBWB, Start of Green

Control Type: Pretimed

Maximum v/c Ratio: 2.60

Intersection Signal Delay: 323.5

Intersection Capacity utilization 135.18

Analysis Period (min) 15

- Volume exceeds capacity, queue is theoretically infinite. Queue shown is maxinum after two cycles.

\# 95th percentile volume exceeds capacity, queue may be longer.
$72.0 \quad 72.0$

2.02 .0

70.0

0.43

368.0

0.0

368.0

368.0

F

1495

16346

3181

3791

$\sim 631.9$

\#667.1

828.0

1438

0

0

1.75
5.0

5.0

134.0

0.83

0.52

3.5

0.0

3.5

3.5

A.

223

18

337

66

78

0

32.4

37.8

83.4

2236

0

0

0

0.52
2.2

$\begin{array}{lll}2.0 & 72.0 \quad 72.0\end{array}$

\begin{tabular}{|c|c|c|c|c|c|c|c|c|c|c|c|}
\hline EBL & $\mathrm{EBT}$ & EBR & WBL & WBT & WBR & NBL & NBT & NBR & SBL & SBT & SBR \\
\hline 0 & $\langle 2\rangle$ & 0 & 0 & $\langle 2\rangle$ & 0 & 0 & $\langle 2\rangle$ & 0 & 0 & $\langle 2\rangle$ & 0 \\
\hline 10 & 1910 & 389 & 257 & 777 & 33 & 350 & 10 & 273 & 29 & 94 & 31 \\
\hline 0 & $\begin{array}{l}3489 \\
0.947\end{array}$ & 0 & 0 & $\begin{array}{l}3518 \\
0.529\end{array}$ & 0 & 0 & $\begin{array}{l}3256 \\
0.673\end{array}$ & 0 & 0 & $\begin{array}{l}3440 \\
0.591\end{array}$ & 0 \\
\hline 0 & $\begin{array}{l}3304 \\
18\end{array}$ & 0 & 0 & $\begin{array}{l}1884 \\
8\end{array}$ & 0 & 0 & $\begin{array}{l}2252 \\
92\end{array}$ & 0 & 0 & $\begin{array}{l}2051 \\
15\end{array}$ & 0 \\
\hline 0 & 2510 & 0 & 0 & 1160 & 0 & 0 & 688 & 0 & 0 & 168 & 0 \\
\hline cust & & & cust & & & cust & & & cust & & \\
\hline
\end{tabular}

\section{$\begin{array}{lll}3 & 3\end{array}$}

4.0

18.0

5.0

18.0

5.0

13.0

0.08

2.60

750.6

0.0

750.6

F

750.6

E

291

384

7106

1383

1648

0

$\sim 181.6$

$\# 222.2$

189.0

\section{5}

0

0

0

2.60
3

$18.0 \quad 0.0$ $5.0 \quad 4.0$

13.0

0.08

0.94

119.8

0.0

119.8

F

119.8

E

124

18

331

64

77

0

26.3

$\# 51.4$

87.2

178

0

0

0.94
Intersection LOS: $\mathbf{F}$

ICU Level of Service $H$ 
Lanes, Volumes, Timings 5: O'Connor Don Milis

\section{Lane Group}

Lane Configurations

Volume (vph)

Satd. Flow (prot)

Flt Permitted

Satd. Flow (perm)

Satd. Flow (RTOR)

Lane Group Flow (vph)

Turn Type

Protected Phases

Permitted Phases

Total Split (s)

Total Lost Time (s)

Act Effct Green (s)

Actuated $g / C$ Ratio

$v / c$ Ratio

Control Delay

Queue Delay

Total Delay

LOS

Approach Delay

Approach LOS

Stops (vph)

Fuel Used(1)

CO Emissions $(\mathrm{g} / \mathrm{hr})$

NOx Emissions $(g / h r)$

VoC Emissions $(g / h r)$

Dilemma Vehicles (\#)

Queue Length 50th (m)

Queue Length 95 th (m)

Internal Link Dist (m)

Turn Bay Length (m)

Base Capacity (vph)

Starvation Cap Reductn

Spillback Cap Reductn

Storage Cap Reductn

Reduced $v / c$ Ratio

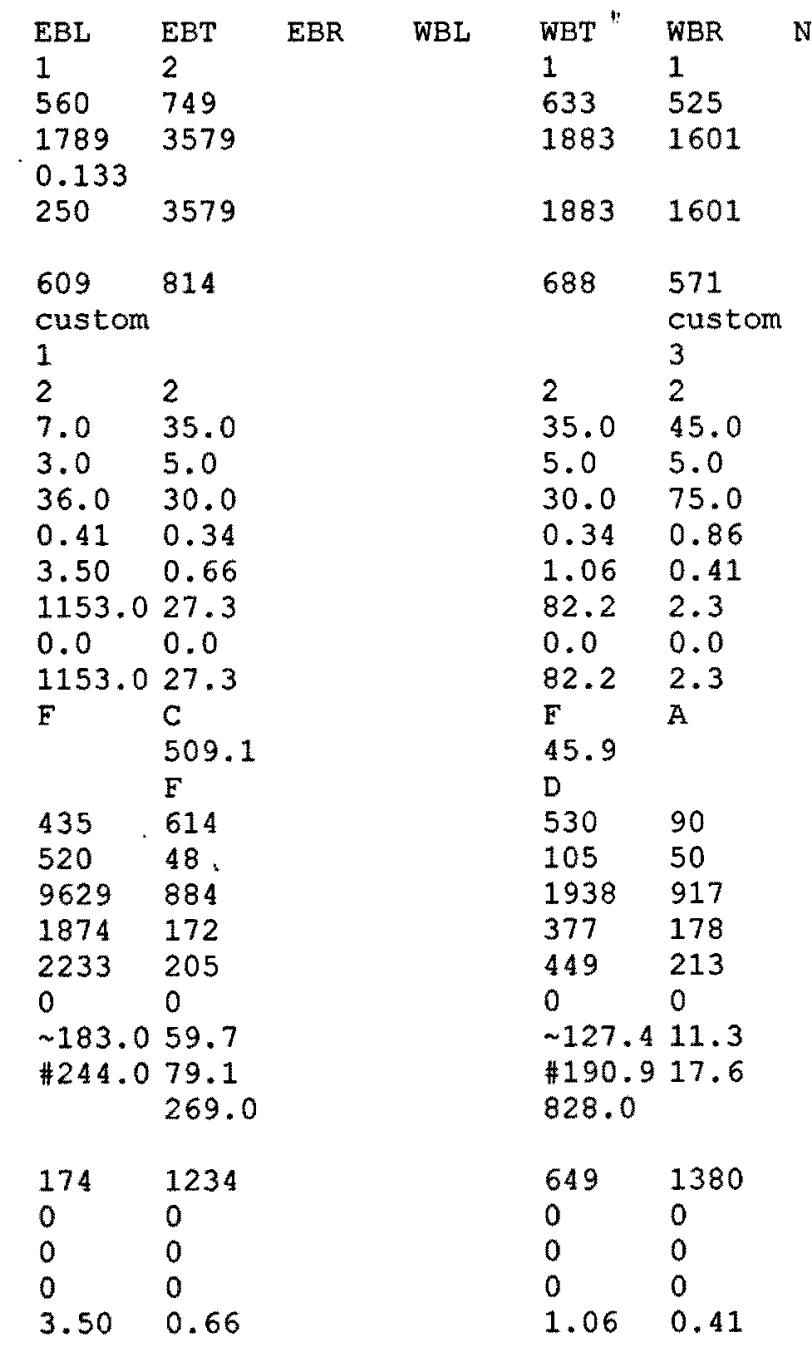

NBL NBT NBR

$\begin{array}{lll}\text { SBL } & \text { SBT } & \text { SBR } \\ 2 & 1 \\ 1560 & & 394 \\ 3471 & 1601 \\ 0.950 & \\ 3471 & 1601 \\ & 76 \\ 1696 & 428 \\ & \text { custom } \\ & 1 \\ 3 & 3 \\ 45.0 & 7.0 \\ 5.0 & 3.0 \\ 40.0 & 49.0 \\ 0.46 & 0.56 \\ 1.06 & 0.46 \\ 66.0 & 10.9 \\ 0.0 & 0.0 \\ 66.0 & 10.9 \\ \text { E } & \text { B } \\ 54.9 & \end{array}$

Intersection Sumary

Cycle Length: 87

Actuated Cycle Length: 87

Offset: $20(238)$, Referenced to phase $2:$ EBWB and $6:$, start of Green

Control Type: Pretimed

Maximum v/c Ratio: 3.50

Intersection Signal Delay: 187.0

Intersection LOS: $\mathrm{F}$

Intersection Capacity utilization $120.5 \%$

ICU Level of Service $H$

Analysis Period (min) 15

Volume exceeds capacity, queue is theoretically infinite.

Queue shown is maximum after two cycles.

\# 95th percentile volume exceeds capacity, queue may be longer. 
Lanes, Volumes, Timings

6: O'Connor \& Donlands

$4 / 2 / 2007$

Lane Group

Lane Configurations

Volume (vph)

Satd. Flow (prot)

Flt Permitted

satd. Flow (perm)

Satd. Flow (RTOR)

Lane Group Flow (vph)

Turn Type

Protected Phases

Permitted Phases

Total Split (s)

Total Lost Time (s)

Act Effct Green (s)

Actuated g/C Ratio

v/c Ratio

Control Delay

Queue Delay

Total Delay

LOS

Approach Delay

Approach LOS

Stops (vph)

Fuel Used(1)

CO Emissions ( $g / h r)$

NOx Emissions $(g / h r)$

VOC Emissions $(g / h r)$

Dilemma Vehicles (\#)

Queue Length 50th (m)

Queue Length 95th (m)

Internal Link Dist (m)

Turn Báy Length (m)

Base Capacity (vph)

Starvation Cap Reductn

Spillback Cap Reductn

Storage Cap Reductn

Reduced v/c Ratio

\begin{tabular}{|c|c|c|c|c|c|c|c|c|c|c|c|}
\hline EBL & EBT & EBR & WBL & WBT & WBR & NBL & NBT & NBR & SBL & SBT & SBR \\
\hline 0 & $<1$ & 1 & 0 & $<1$ & 1 & 0 & $\langle 2\rangle$ & 0 & 0 & $<1$ & 1 \\
\hline 10 & 580 & 44 & 316 & 553 & 158 & 76 & 298 & 385 & 344 & 605 & 20 \\
\hline 0 & $\begin{array}{l}1882 \\
0.580\end{array}$ & 1601 & 0 & $\begin{array}{l}1850 \\
0.097\end{array}$ & 1601 & 0 & $\begin{array}{l}3290 \\
0.497\end{array}$ & 0 & 0 & $\begin{array}{l}1850 \\
0.318\end{array}$ & 1601 \\
\hline 0 & 1092 & $\begin{array}{l}1601 \\
35\end{array}$ & 0 & 183 & $\begin{array}{l}1601 \\
144\end{array}$ & 0 & $\begin{array}{l}1643 \\
262\end{array}$ & 0 & 0 & 599 & $\begin{array}{l}1601 \\
12\end{array}$ \\
\hline & 641 & $\begin{array}{l}48 \\
\text { custo }\end{array}$ & $\begin{array}{l}0 \\
\text { cus }\end{array}$ & 944 & $\begin{array}{l}172 \\
\text { custo }\end{array}$ & $\begin{array}{l}0 \\
\text { cust }\end{array}$ & 825 & 0 & $\begin{array}{l}0 \\
\text { cust }\end{array}$ & 1032 & $\begin{array}{l}22 \\
\text { custo }\end{array}$ \\
\hline
\end{tabular}

$\begin{array}{llll}1 & 1 & 1\end{array}$

$\begin{array}{llllllll}2 & 2 & 2 & 1 & 1 & 1 & 3 & 3 \\ 33.0 & 33.0 & 33.0 & 33.0 & 33.0 & 33.0 & 47.0 & 47\end{array}$

$\begin{array}{llllllll}33.0 & 33.0 & 33.0 & 33.0 & 33.0 & 33.0 & 47.0 & 47.0\end{array}$

47.0

$\begin{array}{llll}0.0 & 3 & 3 & 3\end{array}$

$\begin{array}{lllll}28.0 & 28.0 & 60.0 & 63.0 & 42.0\end{array}$

$\begin{array}{llll}4.0 & 5.0 & 5.0 & 5.0\end{array}$

$\begin{array}{lllll}0.25 & 0.25 & 0.53 & 0.56 & 0.37\end{array}$

$\begin{array}{lllll}2.37 & 0.11 & 1.75 & 0.18 & 1.06\end{array}$

$\begin{array}{lllll}647.9 & 15.5 & 370.2 & 3.4 & 75.5\end{array}$

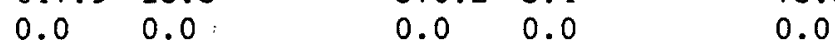

$\begin{array}{lllll}647.9 & 15.5 & 370.2 & 3.4 & 75.5\end{array}$

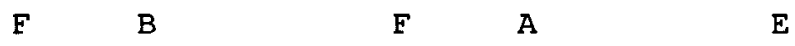

$\begin{array}{lll}603.9 & 313.7 & 75.5 \\ \mathrm{~F} & \mathrm{~F} & \mathrm{E}\end{array}$

$\begin{array}{lllll}\mathrm{F} & & \mathrm{F} & \mathrm{E} \\ 391 & 16 & 556 & 18 & 488\end{array}$

$\begin{array}{lllll}306 & 16 & 556 & 18 & 48 \\ 5 & 1 & 282 . & 6 & 86\end{array}$

$\begin{array}{lllll}5652 & 22 & 5220 & 103 & 1589\end{array}$

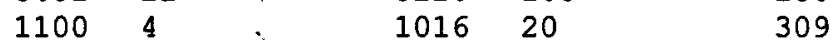

$\begin{array}{lllll}1311 & 5 & 1211 & 24 & 369\end{array}$

$\begin{array}{lllll}0 & 0 & 0 & 0 & 0\end{array}$

$\sim 232.92 .2 \quad \sim 302.22 .6 \quad \sim 85.5$

\#300.5 11.5 \#378.1 12.2 \#124.5

67.7

269.0

409.0

$\begin{array}{ll}271 & 423 \\ 0 & 0 \\ 0 & 0 \\ 0 & 0 \\ 2.37 & 0.11\end{array}$

$\begin{array}{ll}540 & 956 \\ 0 & 0 \\ 0 & 0 \\ 0 & 0 \\ 1.75 & 0.18\end{array}$

775
0
0
0
1.06

$42.0 \quad 42.0$

$0.37 \quad 0.37$

4.630 .04

1654.614 .7

$0.0 \quad 0.0$

1654.614 .7

F B

1620.3

F

$824 \quad 8$

$1230 \quad 1$

$22757 \quad 10$

$4428 \quad 2$

52772

0

$\sim 382.51 .4$

$\# 459.96 .6$

75.2

Intersection Summary

Cycle Length: 113

Actuated Cycle Length: 113

Offset: $0(0 \%)$, Referenced to phase 2:EBWB, start of Green

Control Type: Pretimed

Maximum v/c Ratio: 4.63

Intersection Signal Delay: 688.5

Intersection Capacity Utilization 167.28

Intersection LOS: $F$

Analysis Period (min) 15

Volume exceeds capacity, queue is theoretically infinite.

ICU Level of Service $\mathrm{H}$

$\begin{array}{ll}223 & 603 \\ 0 & 0 \\ 0 & 0 \\ 0 & 0 \\ 4.63 & 0.04\end{array}$

Queue shown is maximum after two cycles.

\# 95th percentile volume exceeds capacity, queue may be longer. 
Lanes, Volumes, Timings

7: Cosburn \& Donlands

$4 / 2 / 2007$

\section{Lane Group}

Lane Configurations

Volume (vph)

Satd. Flow (prot)

Flt Permitted

Satd. Flow (perm)

Satd. Flow (RTOR)

Lane Group Flow (vph)

Turn Type

Protected Phases

Permitted Phases

Total Split (s)

Total Lost Time (s)

Act Effet Green (s)

Actuated g/C Ratio

$v / c$ Ratio

Control Delay

Queue Delay

Total Delay.

LOS

Approach Delay

Approach LOS

Stops (vph)

Fuel Used(1)

CO Emissions $(\mathrm{g} / \mathrm{hr})$

Nox Emissions ( $g / h r)$

VOC Emissions $(g / h r)$

Dilemma Vehicles (\#)

Queue Length 50th (m)

Queue Length 95th (m)

Internal Link Dist (m)

Turn Bay Length (m)

Base Capacity (vph)

Starvation Cap Reductn

Spillback Cap Reductn

Storage Cap Reductn

Reduced v/c Ratio

\begin{tabular}{|c|c|c|c|c|c|c|c|c|c|c|c|}
\hline EBL & EBT & EBR & WBL & WBT & WBR & NBL & NBT & NBR & SBL & SBT & SBR \\
\hline 1. & 1 & 1 & 1 & 1> & 0 & 0 & $\langle 2\rangle$ & 0 & 0 & $\langle 2\rangle$ & 0 \\
\hline 42 & 349 & 49 & 68 & 240 & 119 & 17 & 600 & 91 & 146 & 707 & 112 \\
\hline $\begin{array}{l}1789 \\
0.377\end{array}$ & 1883 & 1601 & $\begin{array}{l}1789 \\
0.389\end{array}$ & 1789 & 0 & 0 & $\begin{array}{l}3507 \\
0.917\end{array}$ & 0 & 0 & $\begin{array}{l}3490 \\
0.639\end{array}$ & 0 \\
\hline 710 & 1883 & $\begin{array}{l}1601 \\
53\end{array}$ & 733 & $\begin{array}{l}1789 \\
32\end{array}$ & 0 & 0 & $\begin{array}{l}3219 \\
25\end{array}$ & 0 & 0 & $\begin{array}{l}2248 \\
22\end{array}$ & 0 \\
\hline 46 & 379 & 53 & 74 & 390 & 0 & 0 & 769 & 0 & 0 & 1049 & 0 \\
\hline
\end{tabular}

$\begin{array}{lllll}2 & 2 & 2 & 2 & 2 \\ 41.0 & 41.0 & 41.0 & 41.0 & 41.0 \\ 5.0 & 5.0 & 5.0 & 5.0 & 5.0 \\ 36.0 & 36.0 & 36.0 & 36.0 & 36.0 \\ 0.39 & 0.39 & 0.39 & 0.39 & 0.39 \\ 0.17 & 0.51 & 0.08 & 0.26 & 0.54 \\ 20.3 & 24.4 & 5.6 & 22.1 & 23.1 \\ 0.0 & 0.0 & 0.0 & 0.0 & 0.0 \\ 20.3 & 24.4 & 5.6 & 22.1 & 23.1 \\ \mathrm{C} & \mathrm{C} & \mathrm{A} & \mathrm{C} & \mathrm{C} \\ & 21.9 & & & 23.0 \\ & \mathrm{C} & & & \mathrm{C} \\ 28 & 259 & 9 & 46 & 249 \\ 1 & 14 . & 1 & 4 & 23 \\ 27 & 254 & 15 & 79 & 425 \\ 5 & 49 & 3 & 15 & 83 \\ 6 & 59 & 4 & 18 & 99 \\ 0 & 0 & 0 & 0 & 0 \\ 5.2 & 50.1 & 0.0 & 8.7 & 47.9 \\ 12.9 & 76.1 & 6.8 & 19.4 & 75.1 \\ & 69.7 & & & 319.0 \\ 278 & 737 & 659 & 287 & 720 \\ 0 & 0 & 0 & 0 & 0 \\ 0 & 0 & 0 & 0 & 0 \\ 0 & 0 & 0 & 0 & 0 \\ 0.17 & 0.51 & 0.08 & 0.26 & 0.54\end{array}$

11

0.0

51.

1.0

11

$51.0 \quad 51.0 \quad 0.0$

5.0

5.0

4.0

5.0

4.0

46.0

0.50

0.47

46.0

0.50

0.92

15.7

0.0

15.7

B

35.9

0.0

35.9

15.7

B

426

35.9

D

797

82

1524

426

297

353

99

0

43.2

0

58.0

$\# 131.7$

409.0

Intersection Summary

Cycle Length: 92

Actuated Cycle Length: 92

Offset: $0(0 \%)$, Referenced to phase 2 :EBWB and $6:$, Start of Green

Control Type: Pretimed

Maximum v/c Ratio: 0.92

Intersection Signal Delay: 25.7

Intersection LOS: C

Intersection Capacity Utilization 87.28

ICU Level of Service E

Analysis Period (min) 15

\# 95th percentile volume exceeds capacity, queue may be longer.

Queue shown is maximum after two cycles.

1135

0

0.92 
Lane Group

Lane Configurations

volume (vph)

satd. Flow (prot)

Flt Permitted

Satd. Flow (perm)

satd. Flow (RTOR)

Lane Group Elow (vph)

Turn Type

Protected Phases

Permitted Phases

Total Split (s)

Total Lost Time (s)

Act Effct Green (s)

Actuated $g / C$ Ratio

v/C Ratio

Control Delay

Queue Delay

Total Delay

LOS

Approach Delay

Approach LOS

Stops (vph)

Fuel Used(1)

CO Emissions ( $g / h r)$

NOx Emissions $(g / h r)$

VOC Emissions $(g / h r)$

Dilemma Vehicles (\#)

Queue Length 50th (m)

Queue Length 95th (m)

Internal Link Dist (m)

Turn Bay Length (m)

Base Capacity (vph)

Starvation Cap Reductn

Spillback Cap Reductn

Storage Cap Reductn

Reduced v/C Ratio

\begin{tabular}{|c|c|c|c|c|c|c|c|c|c|c|c|}
\hline EBL & EBT & EBR & WBL & WBT & WBR & NBL & NBT & NBR & SBL & SBT & SBR \\
\hline 1 & 1> & 0 & 1 & 1> & 0 & 1 & 1) & 0 & 0 & $\langle 1\rangle$ & 0 \\
\hline 33 & 542 & 11 & 48 & 297 & 10 & 99 & 40 & 93 & 32 & 333 & 31 \\
\hline $\begin{array}{l}1789 \\
0.502\end{array}$ & 1878 & 0 & $\begin{array}{l}1789 \\
0.283\end{array}$ & 1874 & 0 & $\begin{array}{l}1789 \\
0.377\end{array}$ & 1686 & 0 & 0 & $\begin{array}{l}1855 \\
0.967\end{array}$ & 0 \\
\hline 945 & $\begin{array}{l}1878 \\
2\end{array}$ & 0 & 533 & $\begin{array}{l}1874 \\
3\end{array}$ & 0 & 710 & $\begin{array}{l}1686 \\
101\end{array}$ & 0 & 0 & $\begin{array}{l}1801 \\
5\end{array}$ & 0 \\
\hline $\begin{array}{l}36 \\
\text { custom }\end{array}$ & 601 & 0 & $\begin{array}{l}52 \\
\text { custor }\end{array}$ & 334 & 0 & $\begin{array}{l}108 \\
\text { custor }\end{array}$ & 144 & 0 & $\begin{array}{l}0 \\
\text { cus }\end{array}$ & 431 & 0 \\
\hline
\end{tabular}

$\begin{array}{llllll}2 & 2 & & 2 & 2 & \\ 52.0 & 52.0 & 0.0 & 52.0 & 52.0 & 0.0 \\ 5.0 & 5.0 & 4.0 & 5.0 & 5.0 & 4.0 \\ 47.0 & 47.0 & & 47.0 & 47.0 & \\ 0.52 & 0.52 & & 0.52 & 0.52 & \\ 0.07 & 0.61 & & 0.19 & 0.34 & \end{array}$

11

$38.0 \quad 38.0$

$\begin{array}{lll}0 & 1 & 1\end{array}$

$\begin{array}{lll}5.0 & 5.0 & 4.0\end{array}$

38.0
5.0

38.0

0.0

0.37

3.0

0.37

$0.42 \quad 0.21$

0.37
0.65

$\begin{array}{llll}11.3 & 18.4 & 13.6 & 13.6\end{array}$

$\begin{array}{llll}0.0 & 0.0 & 0.0 & 0.0\end{array}$

$11.3 \quad 18.4$

$13.6 \quad 13.6$

B $B$

$38.7 \quad 21.0$

28.9

$0.0 \quad 0.0$

$38.7 \quad 21.0$

D

0.0

B $B$

13.6

$\mathrm{B}$

$16 \quad 374$

233

$32 \quad 616$

$6 \quad 120$

$7 \quad 143$

00.

$2.9 \quad 68.8$

$7.6 \quad 101.7$

319.0

$\mathrm{B}$

$26 \quad 168$

210

$29 \quad 187$

$6 \quad 36$

$7 \quad 43$

$0 \quad 0$

$4.5 \quad 31.4$

$11.3 \quad 48.9$

113.8

28.6

28.9

$\mathrm{C}$

28.9

$\mathrm{C}$

320

$92 \quad 132$

810

$156 \quad 178$

$30 \quad 35$

$36 \quad 41$

$0 \quad 0$

18.514 .6

$\mathrm{m} 24.3 \mathrm{~m} 20.6$

4.0

$\begin{array}{ll}494 . & 982 \\ 0 & 0 \\ 0 & 0 \\ 0 & 0 \\ 0.07 & 0.61\end{array}$

$\begin{array}{ll}278 & 980 \\ 0 & 0 \\ 0 & 0 \\ 0 & 0 \\ 0.19 & 0.34\end{array}$

$\begin{array}{ll}260 & 682 \\ 0 & 0 \\ 0 & 0 \\ 0 & 0 \\ 0.42 & 0.21\end{array}$

\section{8}

333

65

77

0

60.2

91.7

85.3

664

0

0

0

0.65

Intersection Summary

Cycle Length: 90

Actuated Cycle Length: 90

Offset: $0(08)$, Referenced to phase 2:EBWB and 6:, start of Green

Control Type: Pretimed

Maximum v/c Ratio: 0.65

Intersection Signal Delay: 21.3

Intersection Capacity Utilization 78.28

Intersection LOS: C

Analysis Period (min). 15

ICU Level of Service D

m Volume for 95 th percentile queue is metered by upstream signal. 
Lane Group

Lane Configurations

Volume (vph)

Satd. Flow (prot)

Flt Permitted

Satd. Flow (perm)

Satd. Flow (RTOR)

Lane Group Flow (vph)

Turn Type

Protected Phases

Permitted Phases

Total split (s)

Total lost Time (s)

Act Effct Green (s)

Actuated g/C Ratio

$v / c$ Ratio

Control Delay

Queue Delay

Total Delay

LOS

Approach Delay

Approach LOS

Stops (vph)

Fuel Used (1)

Co Emissions $(g / h r)$

NOx Emissions $(g / h x)$

VOC Emissions ( $g / h r)$

Dilemma Vehicles (\#)

Queue Length 50th (m)

Queue Length 95 th (m)

Internal Link Dist (m)

Turn Bay Length (m)

Base Capacity (voh)

Starvation Cap Reductn

Spillback Cap Reductn

Storage Cap Reductn

Reduced v/C Ratio

$\begin{array}{llllllllllll}\text { EBL } & \text { EBT } & \text { EBR } & \text { WBL } & \text { WBT } & \text { WBR } & \text { NBL } & \text { NBT } & \text { NBR } & \text { SBL } & \text { SBT } & \text { SBR } \\ 0 . & \langle 1\rangle & 0 & 0 & <1> & 0 & 0 & <1> & 0 & 0 & <1> & 0 \\ 31 & 684 & 38 & 20 & 297 & 17 & 34 & 184 & 156 & 166 & 203 & 21 \\ 0 & 1867 & 0 & 0 & 1865 & 0 & 0 & 1769 & 0 & 0 & 1831 & 0 \\ & 0.975 & & & 0.938 & & & 0.929 & & & 0.480 & \\ 0 & 1824 & 0 & 0 & 1754 & 0 & 0 & 1652 & 0 & 0 & 898 & 0 \\ & 5 & & & 5 & & & 40 & & & 3 & 0 \\ 0 & 818 & 0 & 0 & 363 & 0 & 0 & 407 & 0 & 0 & 424 & 0\end{array}$

custom custom

$\begin{array}{lll}2 & 2 & \\ 59.0 & 59.0 & 0.0 \\ 5.0 & 5.0 & 4.0 \\ & 54.0 & \\ & 0.60 \\ & 0.75 \\ & 18.3 \\ & 0.0 \\ & 18.3 \\ & \mathrm{~B} \\ & 18.3 \\ & \mathrm{~B} \\ & 529 \\ & 26 \\ 484 & \\ 94 \\ 112 \\ 0 \\ 93.3 \\ 140.7 \\ 75.6 \\ \\ 1096 \\ 0 \\ 0 \\ 0 \\ 0.75 \\ \end{array}$

$\begin{array}{ll}2 & 2 \\ 59.0 & 59.0 \\ 5.0 & 5.0 \\ & 54.0 \\ & 0.60 \\ & 0.34 \\ 10.1 \\ 0.0 \\ 10.1 \\ \text { B } \\ 10.1 \\ \text { B } \\ 156 \\ 33 \\ 614 \\ 119 \\ 142 \\ 0 \\ 28.6 \\ 44.5 \\ 778.0 \\ 1054 \\ 0 \\ 0 \\ 0 \\ 0.34\end{array}$

custom

custom

Intersection Summary

Cycle Iength: 90

1 Actuated Cycle Length: 90

Offset: $0(08)$, Referenced to phase 2:EBWB and 6:, start of Green

Control Type: Pretimed

Maximum $\mathrm{v} / \mathrm{C}$ Ratio: 1.62

Intersection Signal Delay: 86.5

Intersection Capacity Utilization 102.48

$\begin{array}{lllll}1 & 1 & 1 & 1\end{array}$

\begin{tabular}{|c|c|c|c|c|c|}
\hline 1 & 1 & & 1 & 1 & \\
\hline 31.0 & 31.0 & 0.0 & 31.0 & 31.0 & 0.8 \\
\hline 5.0 & 5.0 & 4.0 & 5.0 & 5.0 & 4. \\
\hline & 26.0 & & & 26.0 & \\
\hline & 0.29 & & & 0.29 & \\
\hline & 0.80 & & & 1.62 & \\
\hline & 40.7 & & & 327.6 & \\
\hline & 0.0 & & & 0.0 & \\
\hline & 40.7 & & & 327.6 & \\
\hline & D & & & $\mathbf{E}$ & \\
\hline & 40.7 & $\infty$ & & 327.6 & \\
\hline & D & & & $\mathbf{E}$ & \\
\hline & 299 & & & 433 & \\
\hline & 21 & & & 121 & \\
\hline & 385 & & & 2238 & \\
\hline & 75 & & & 436 & \\
\hline & 89 & & & 519 & \\
\hline & 0 & & & 0 & \\
\hline & 59.0 & & & -110.5 & \\
\hline o & 104.8 & & & $\# 167.6$ & \\
\hline & 97.1 & & & 375.0 & \\
\hline & 506 & & & 262 & \\
\hline & 0 & & & 0 & \\
\hline & 0 & & & 0 & \\
\hline & 0 & & & 0 & \\
\hline & 0.80 & & & 1.62 & \\
\hline
\end{tabular}

Analysis Period (min) 15

- Volume exceeds capacity, queue is theoretically infinite.

Queue shown is maximum after two cycles.

\# 95th percentile volume exceeds capacity, queue may be longer.

Intersection LOS: $F$

ICU Level of Service $G$ 